Supporting Information for:

\title{
Cooperative Heterobimetallic Zinc/Alkaline Earth Metal Catalysis: A Zn/Sr Aminophenol Sulfonamide Complex for Catalytic Asymmetric Michael Addition of 3-Acetoxy-2- Oxindoles to $\beta$-Ester Enones
}

Yuchen Yang ${ }^{\dagger}$, Cheng Tang ${ }^{\dagger}$, Guojuan Liang ${ }^{\dagger}$, Ping Deng, Jing Zhou, Zinan Yang, Peng Chen, and Hui Zhou*

hzhou@cqmu.edu.cn

College of Pharmacy, Chongqing Medical University, Chongqing, China

\section{CONTENTS}

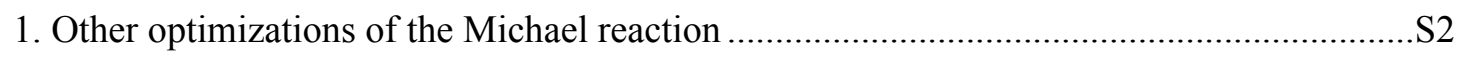

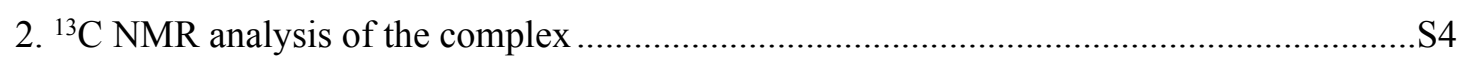

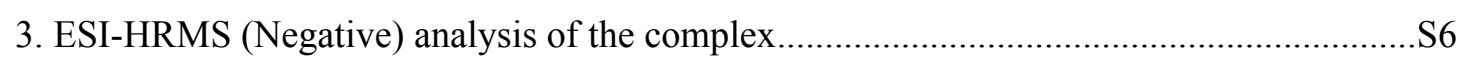

4. The structure of the heterobimetallic $\mathrm{Zn}$-Sr-aminophenol sulfonamide complex optimized

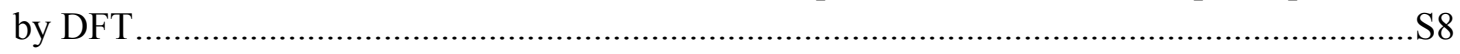

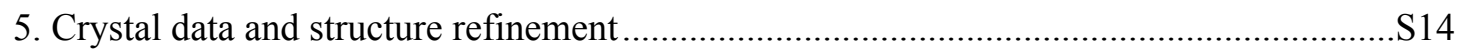

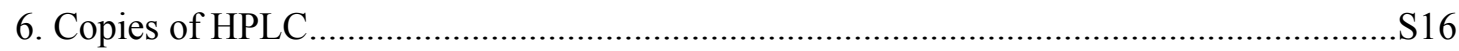

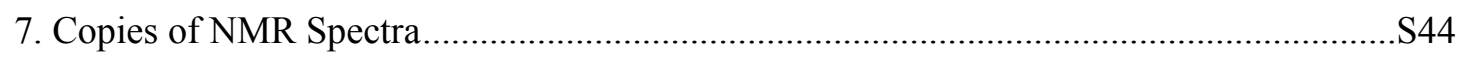




\section{Other optimizations of the Michael reaction}

Table S1: The effects of different ligands. ${ }^{a}$

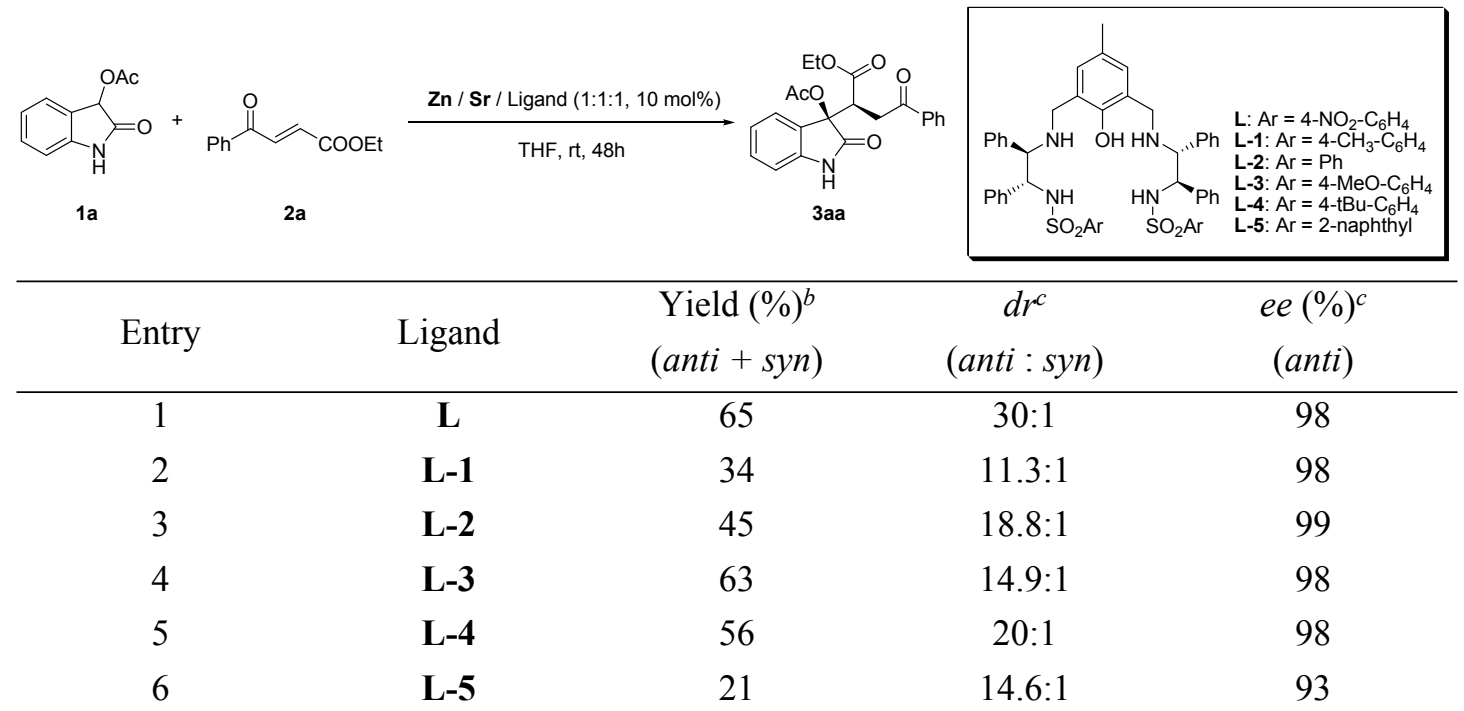

${ }^{a}$ All reactions were performed with $\mathrm{Zn}(\mathrm{acac})_{2} / \mathrm{Sr}(\mathrm{OAc})_{2} / \mathrm{Ligand}(1: 1: 1,10 \mathrm{~mol} \%), \mathbf{1 a}(0.1 \mathrm{mmol})$, 2a $(0.1 \mathrm{mmol})$, THF $(0.5 \mathrm{~mL})$, at $\mathrm{rt}$ for $48 \mathrm{~h} .{ }^{b}$ Isolated yield. ${ }^{c}$ Determined by HPLC.

The ligands were prepared according to the literature. ${ }^{1}$

Table S2: The effects of different solvents.

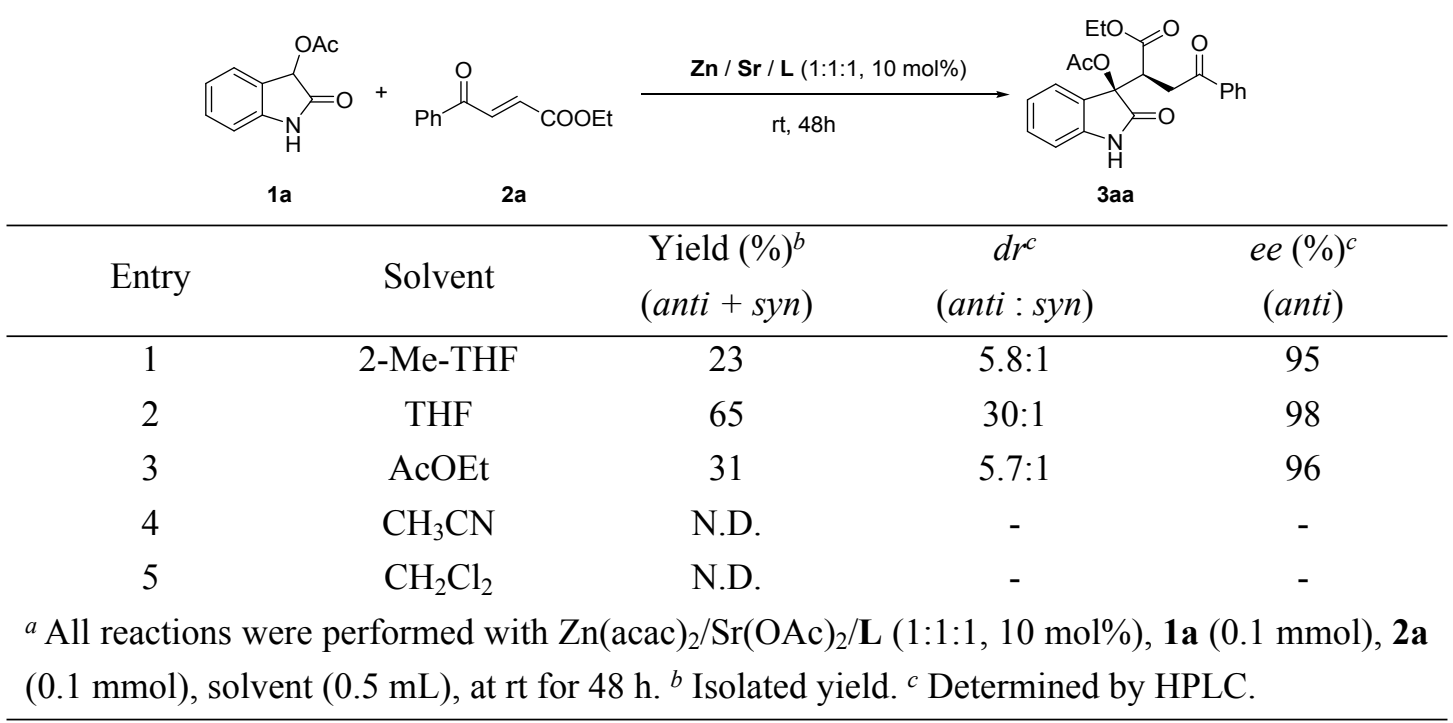

Table S3: Effects of the concentration and the ratio of substrates.

[1] Zhang, S.; Deng, P.; Zhou, J.; Liu, M.; Liang, G.; Xiong, Y.; Zhou, H. Chem. Commun. 2017, 53, 1291412917. 


\begin{tabular}{|c|c|c|c|c|c|}
\hline & $1 \mathrm{a}$ & $2 a$ & & 3aа & \\
\hline Entry & $\begin{array}{l}\text { The amount of } \\
\mathbf{1 a}(\mathrm{mmol})\end{array}$ & $\begin{array}{l}\text { The amount of } \\
\mathbf{2 a}(\mathrm{mmol})\end{array}$ & $\begin{array}{l}\text { Yield }(\%)^{b} \\
(\text { anti }+ \text { syn })\end{array}$ & $\begin{array}{c}d r^{c} \\
(\text { anti }: \text { syn })\end{array}$ & $\begin{array}{r}e e(\%)^{c} \\
(\text { anti) }\end{array}$ \\
\hline $1^{d}$ & 0.1 & 0.1 & 38 & $30: 1$ & 98 \\
\hline 2 & 0.2 & 0.2 & 23 & 17.9:1 & 98 \\
\hline 3 & 0.1 & 0.1 & 65 & $30: 1$ & 98 \\
\hline 4 & 0.1 & 0.12 & 40 & 20:1 & 98 \\
\hline 5 & 0.1 & 0.15 & 44 & $30: 1$ & 98 \\
\hline 6 & 0.1 & 0.2 & 38 & $30: 1$ & 98 \\
\hline 7 & 0.12 & 0.1 & 75 & $20: 1$ & 98 \\
\hline 8 & 0.15 & 0.1 & 58 & 20:1 & 98 \\
\hline 9 & 0.2 & 0.1 & 64 & $20: 1$ & 98 \\
\hline \multicolumn{6}{|c|}{$\begin{array}{l}{ }^{a} \text { Unless otherwise noted, the reactions were performed with } \mathrm{Zn}(\mathrm{acac})_{2} / \mathrm{Sr}(\mathrm{OAc})_{2} / \mathbf{L}(1: 1: 1,10 \\
\mathrm{mol} \%) \text {, THF }(0.5 \mathrm{~mL}) \text {, at } \mathrm{rt} \text { for } 48 \mathrm{~h} .^{b} \text { Isolated yield. }{ }^{c} \text { Determined by HPLC. }{ }^{d} \mathrm{THF}(1.0 \mathrm{~mL}) .\end{array}$} \\
\hline
\end{tabular}

Table S4: The effect of additive.

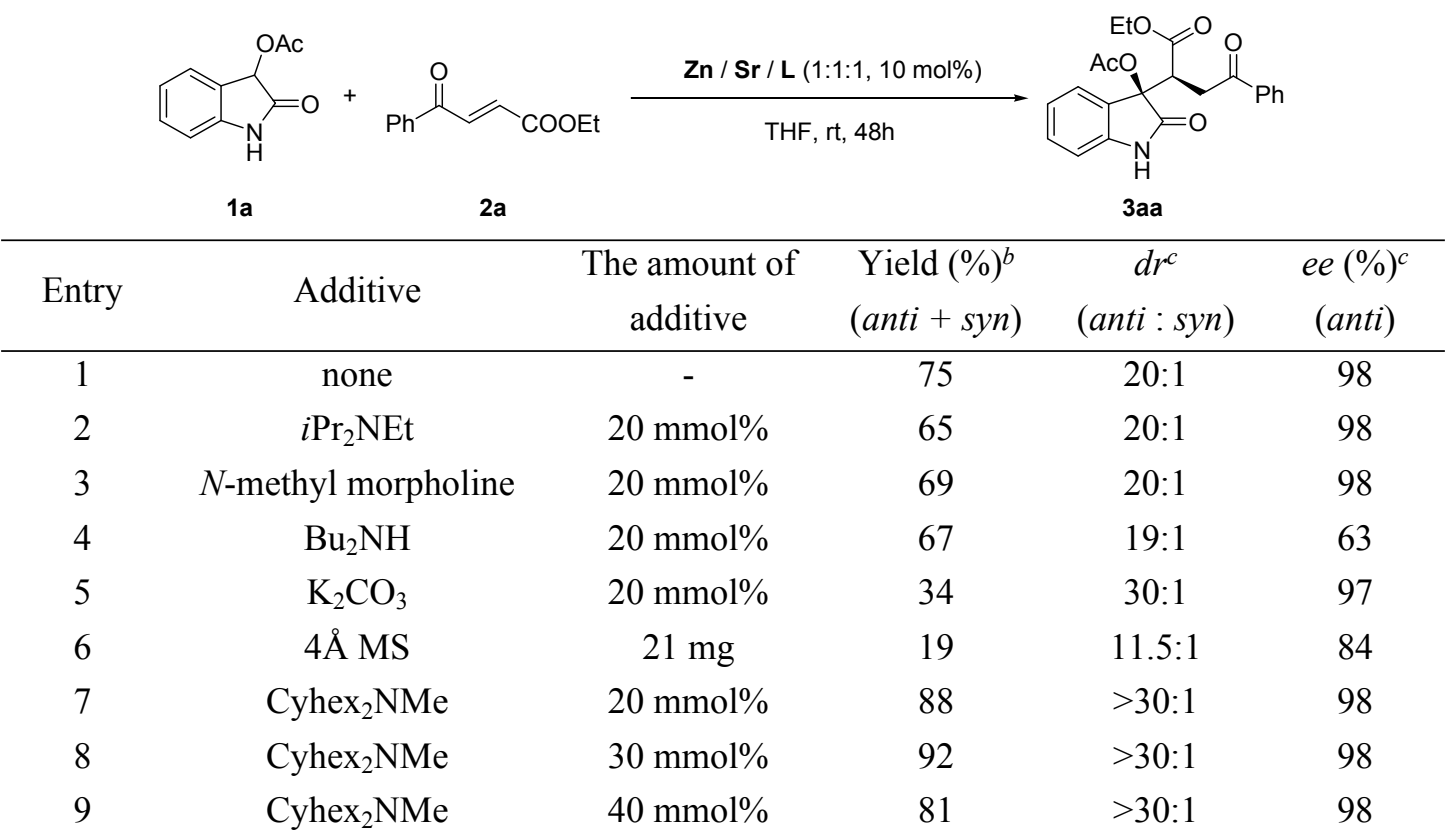

${ }^{a}$ Unless otherwise noted, the reactions were performed with $\mathrm{Zn}(\mathrm{acac})_{2} / \mathrm{Sr}(\mathrm{OAc})_{2} / \mathbf{L}(1: 1: 1,10$ mol\%), 1a $(0.12 \mathrm{mmol}), 2 \mathrm{a}(0.1 \mathrm{mmol}), \mathrm{THF}(0.5 \mathrm{~mL})$, at rt for $48 \mathrm{~h} .{ }^{b}$ Isolated yield. ${ }^{c}$ Determined by HPLC. 
2. ${ }^{13} \mathrm{C}$ NMR analysis of the complex

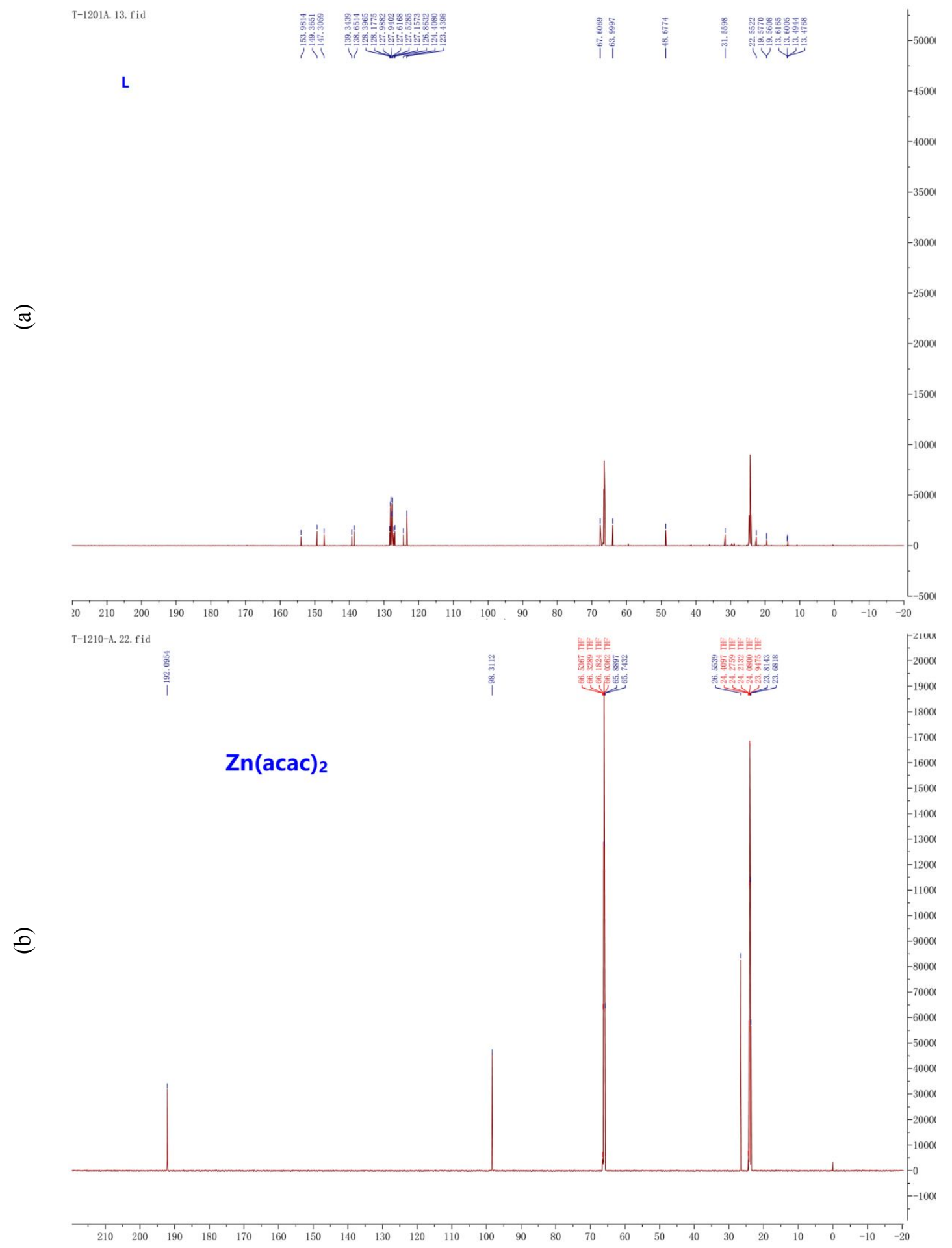




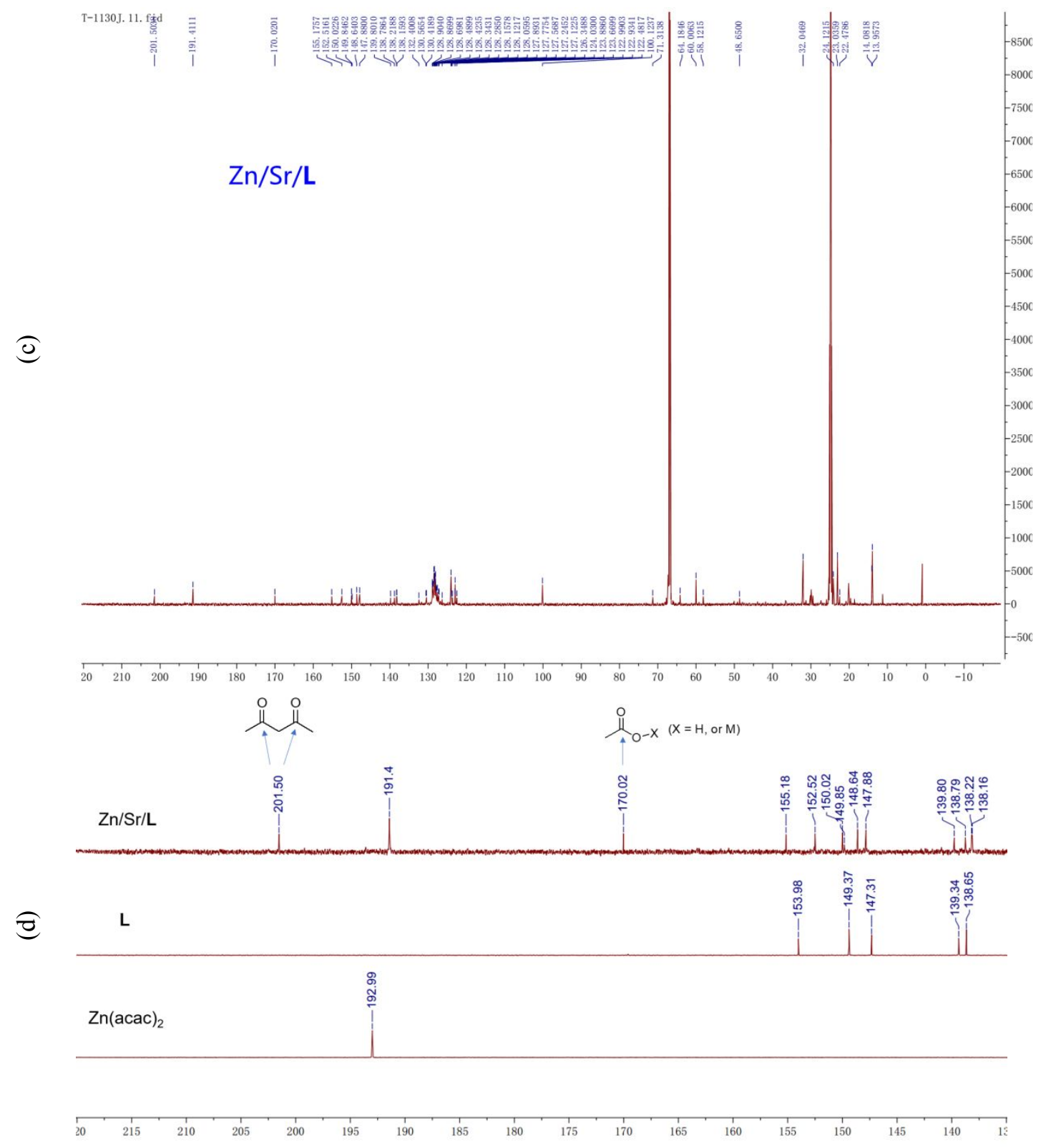

Figure S1. (a) ${ }^{13} \mathrm{C}$ NMR of ligand $\mathbf{L}$ in THF- $d_{8}$; (b) ${ }^{13} \mathrm{C}$ NMR of $\mathrm{Zn}(\mathrm{acac})_{2}$ in THF- $d_{8}$; (c) ${ }^{13} \mathrm{C}$ NMR of $\mathrm{Zn} / \mathrm{Sr} / \mathbf{L}$ complex in THF- $d_{8}$; (d) Screenshot of $\delta 135-220 \mathrm{ppm}$ in a, b and $\mathrm{c}$. 


\section{ESI-HRMS (Negative) analysis of the complex}

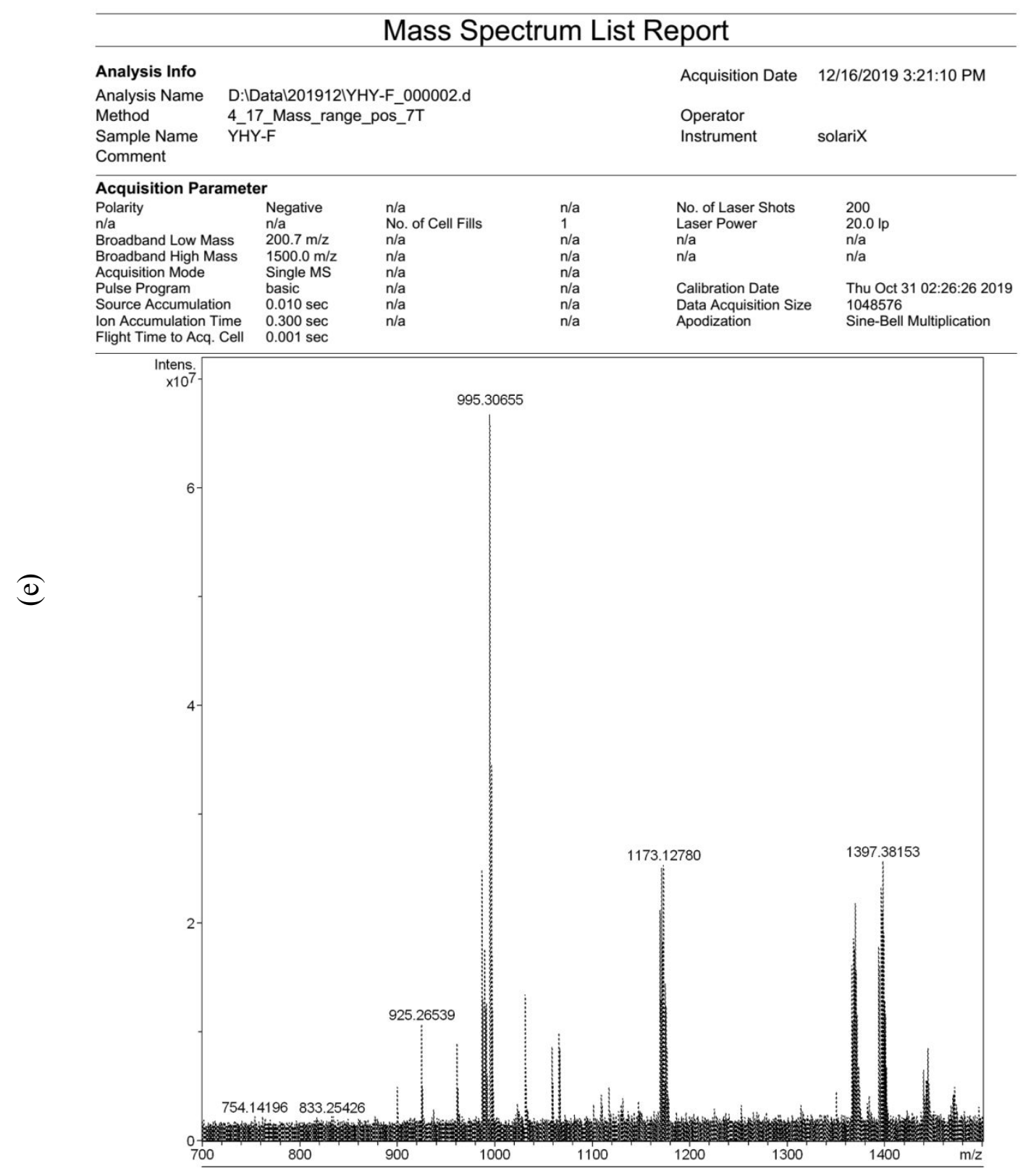

Calcd. $\mathrm{C}_{54} \mathrm{H}_{49} \mathrm{~N}_{6} \mathrm{O}_{11} \mathrm{~S}_{2} \mathrm{SrZn}^{-}\left[\mathrm{M}_{\mathbf{L}}-3 \mathrm{H}+\mathrm{Zn}(\mathrm{acac})+\mathrm{Sr}-\mathrm{H}\right]-: 1173.12538$

Found: 1173.12780

Figure S2. ESI-HRMS of $\mathrm{Zn} / \mathrm{Sr} / \mathrm{L}=1: 1: 1$ mixture (e) 


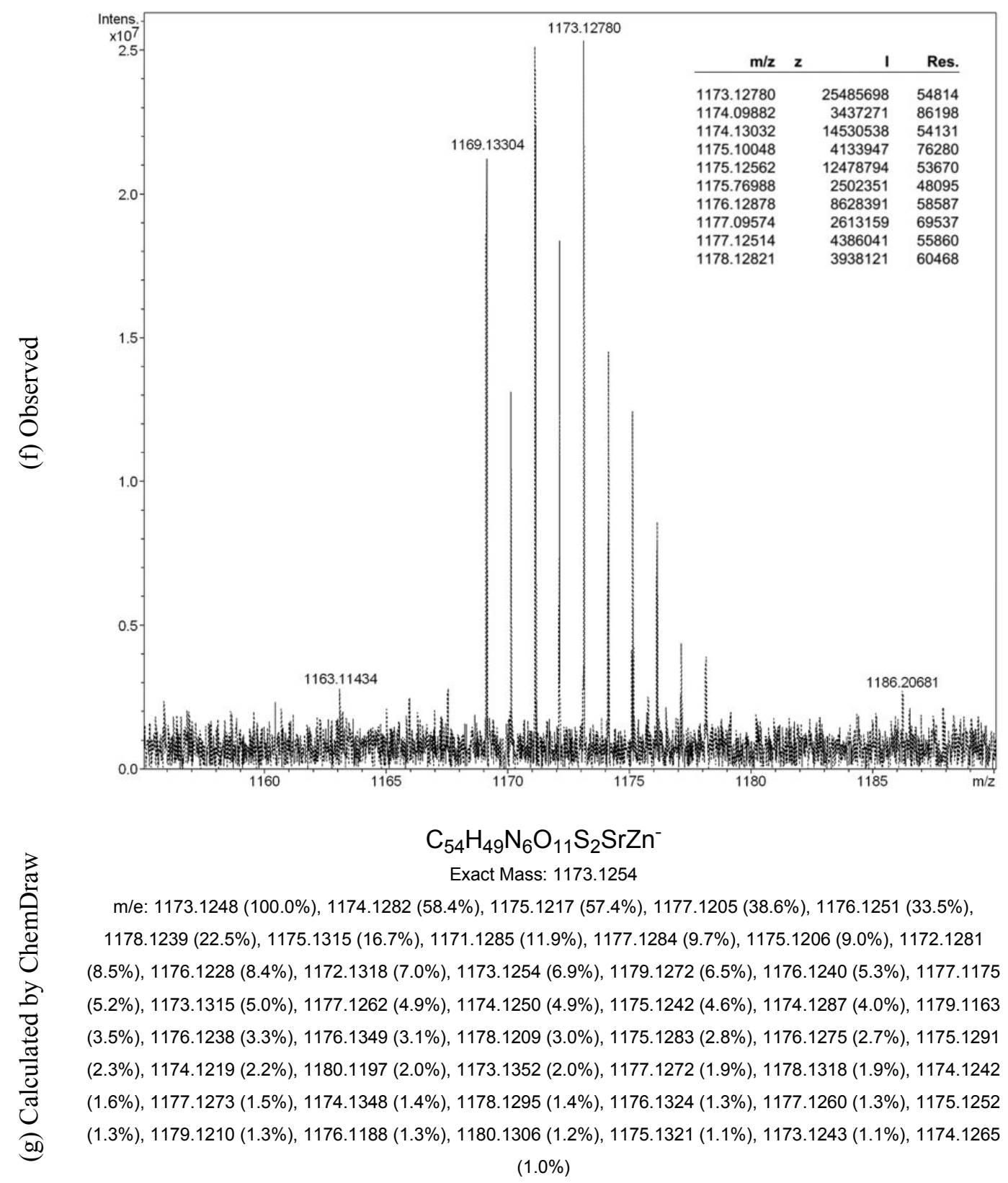




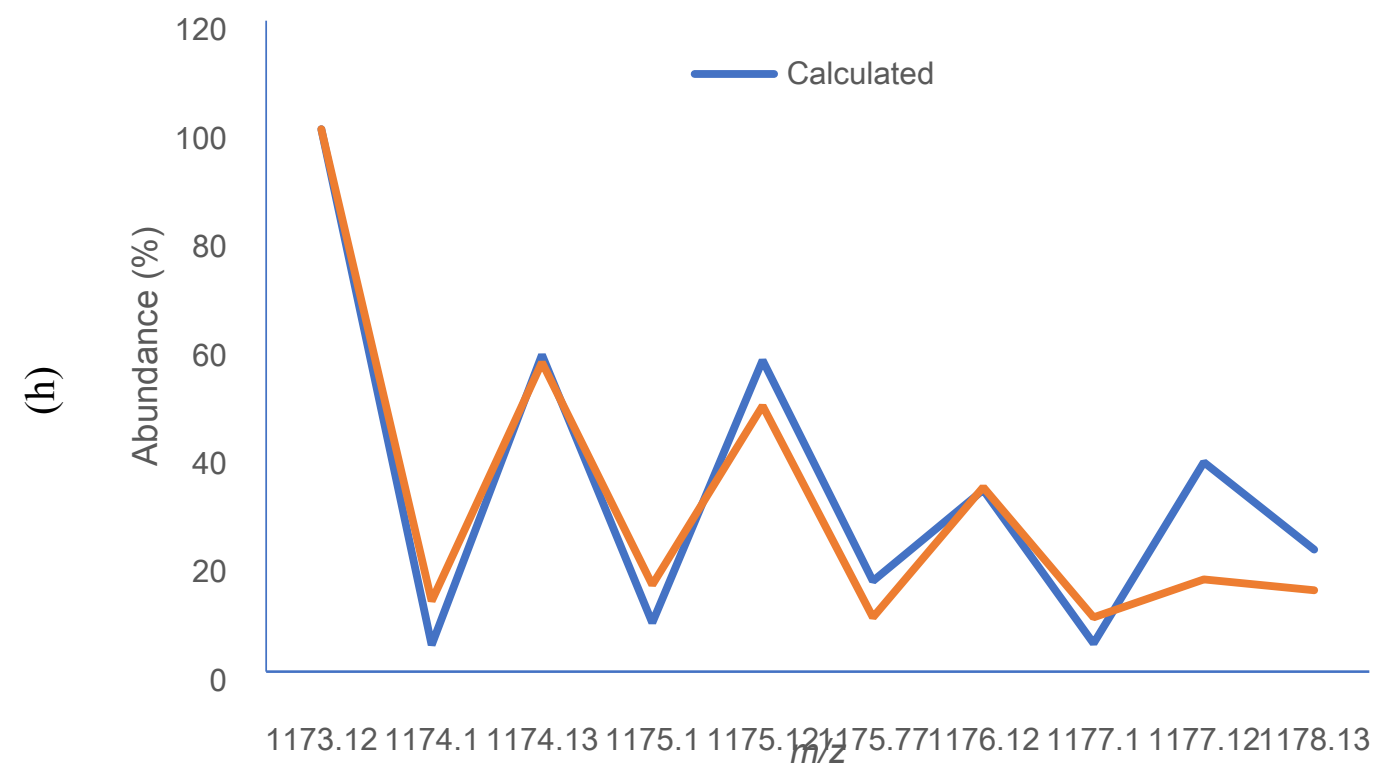

Figure S3. The observed distribution pattern of $\mathrm{m} / \mathrm{z} 1173$ and calculated distribution pattern of $\mathrm{C}_{54} \mathrm{H}_{49} \mathrm{~N}_{6} \mathrm{O}_{11} \mathrm{~S}_{2} \mathrm{SrZn}$ (f, g, h)

4. The structure of the heterobimetallic Zn-Sr-aminophenol sulfonamide complex optimized by DFT

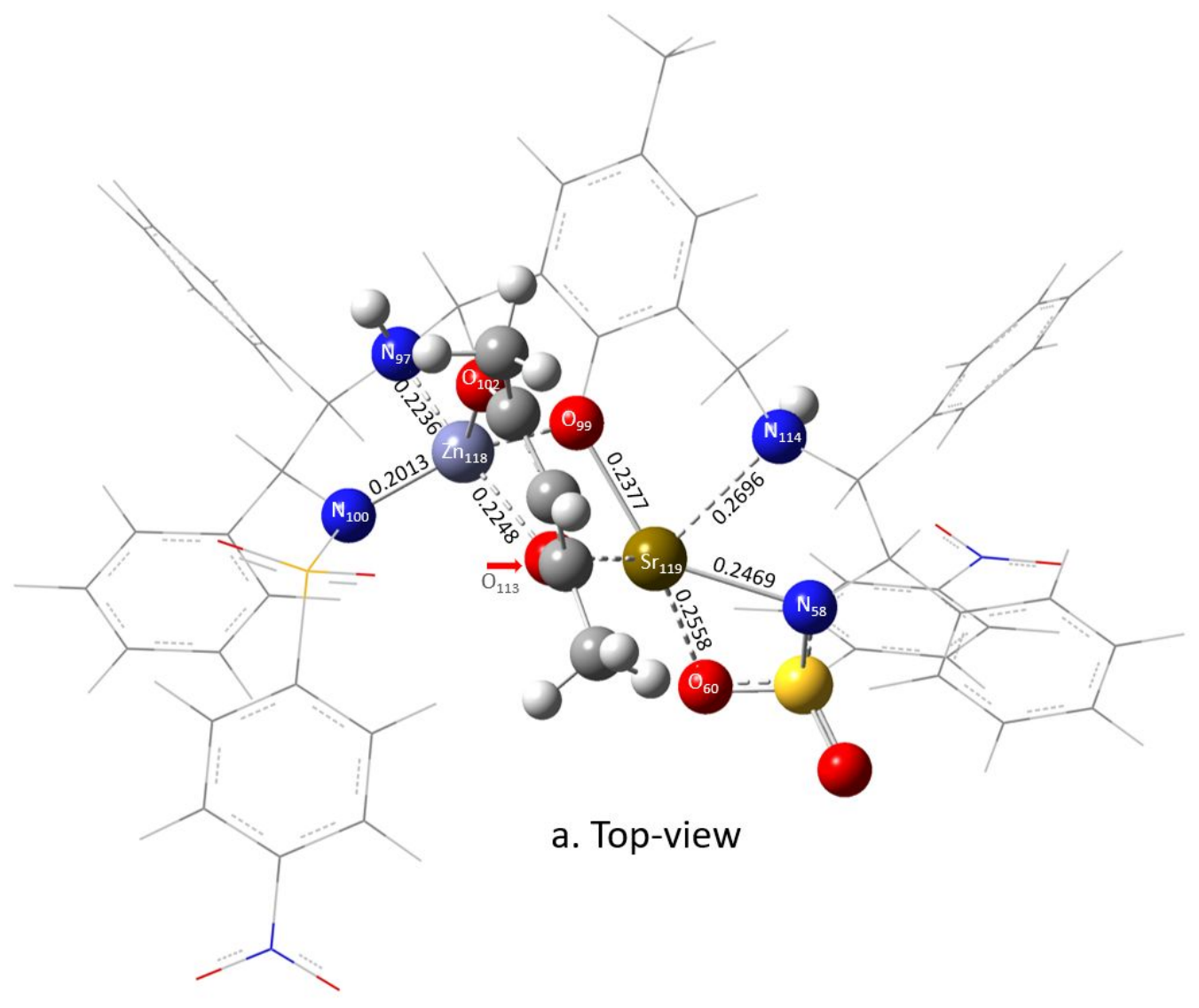




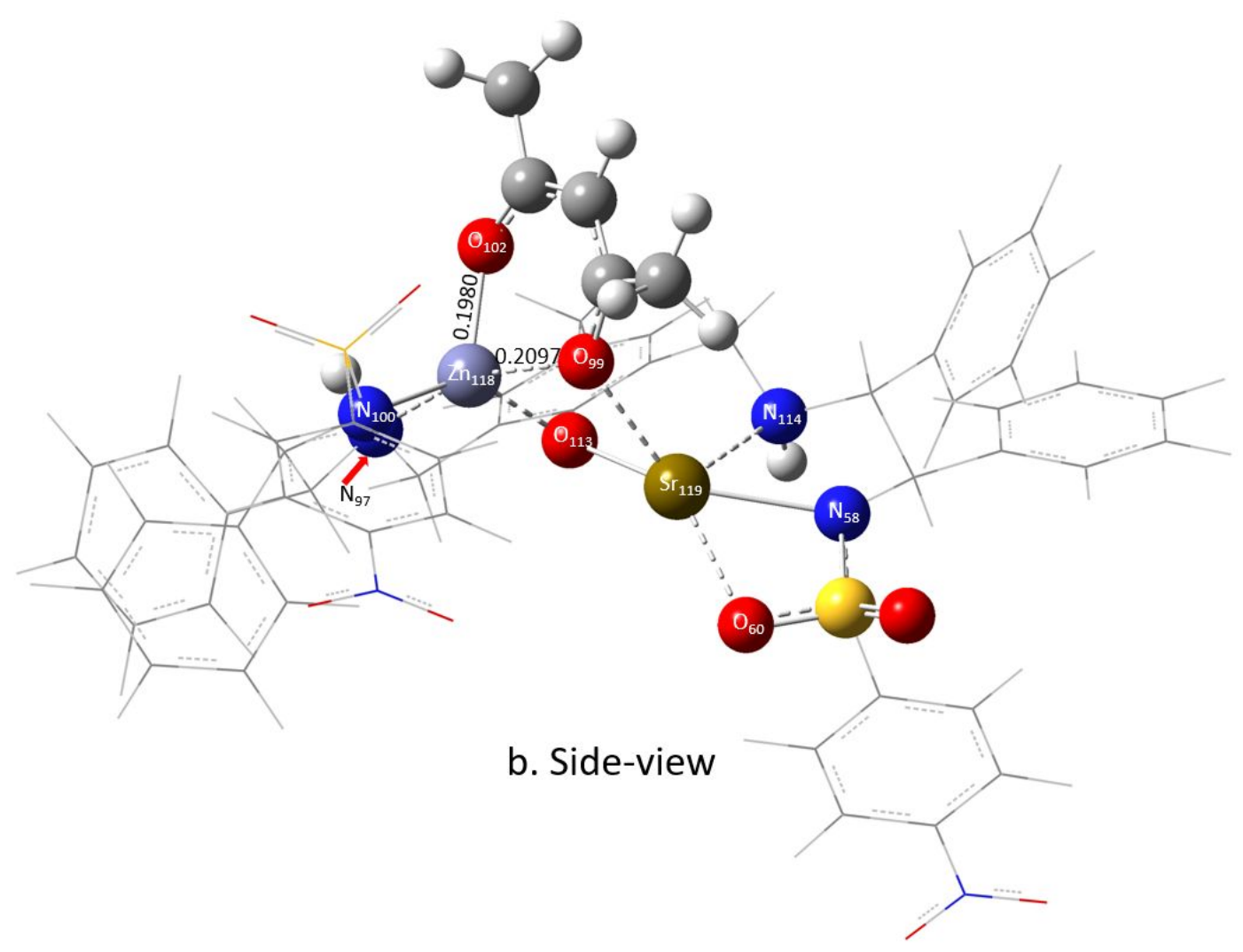

Figure S4. The geometry of $\mathrm{Zn} / \mathrm{Sr} / \mathrm{L}$ (bond length in $\mathrm{nm}$ ) optimized by density functional theory (DFT)

DFT calculations: To determine the coordination environment of the metal ions $\left(\mathrm{Zn}^{2+}\right.$ and $\left.\mathrm{Sr}^{2+}\right)$ within the binuclear complex, density functional theory (DFT) calculations were carried out using the Gaussian 09 package $^{2}$. Becke three-parameter hybrid (B3LYP) exchange correlation function ${ }^{3}$ was performed for the full optimize geometry, 6-31G(d) basis set for C, H, N, O and S atoms, while SDD basis set for Zn and $\mathrm{Sr}$ atoms. Frequency calculation has been done on the optimized geometry to make sure the stable structure by without imaginary frequency.

\section{Cartesian coordinates for the calculated structure}

Standard orientation:

\begin{tabular}{cccccc}
$\begin{array}{l}\text { Center } \\
\text { Number }\end{array}$ & $\begin{array}{c}\text { Atomic } \\
\text { Number }\end{array}$ & Atomic & \multicolumn{2}{c}{ Coordinates (Angstroms) } \\
- & Type & $X$ & $Y$ & $Z$ \\
1 & 6 & 0 & -3.821312 & -1.854593 & 1.282317 \\
2 & 6 & 0 & -4.612394 & -0.913576 & 0.330665
\end{tabular}

[2] Peralta, J.; Ogliaro, F.; Bearpark, M.; Heyd, J.; Brothers, E.; Kudin, K.; Staroverov, V.; Kobayashi, R.; Normand, J.; Raghavachari, K. Gaussian 09, Revision D. 01; Gaussian, Inc.: Wallingford, CT, 2013

[3] Dreuw, A.; Weisman, J. L.; Head-Gordon, M. J. Chem. Phys. 2003, 119, 2943-2946 


\begin{tabular}{|c|c|c|c|c|c|}
\hline 3 & 6 & 0 & 1.403223 & -7.001435 & 0.350962 \\
\hline 4 & 6 & 0 & -1.800082 & -3.244771 & 1.486732 \\
\hline 5 & 6 & 0 & -0.562397 & -3.745614 & 0.796110 \\
\hline 6 & 6 & 0 & 0.246310 & -2.807503 & 0.114677 \\
\hline 7 & 6 & 0 & 1.418421 & -3.262124 & -0.534601 \\
\hline 8 & 6 & 0 & -0.187475 & -5.087642 & 0.852466 \\
\hline 9 & 6 & 0 & 1.764606 & -4.617160 & -0.440533 \\
\hline 10 & 6 & 0 & 2.267395 & -2.348032 & -1.389536 \\
\hline 11 & 6 & 0 & 0.987689 & -5.551634 & 0.249419 \\
\hline 12 & 6 & 0 & 6.225705 & 2.025304 & 3.522935 \\
\hline 13 & 6 & 0 & 4.048293 & -0.597837 & -1.330778 \\
\hline 14 & 6 & 0 & 5.192205 & -1.555736 & -1.644311 \\
\hline 15 & 6 & 0 & 4.534513 & 0.668786 & -0.540563 \\
\hline 16 & 6 & 0 & 5.547427 & 1.463503 & -1.363352 \\
\hline 17 & 6 & 0 & 5.526515 & 1.503029 & 4.609827 \\
\hline 18 & 6 & 0 & 5.835144 & -2.272692 & -0.623539 \\
\hline 19 & 6 & 0 & 5.621599 & -1.746039 & -2.963089 \\
\hline 20 & 6 & 0 & 6.877547 & -3.153042 & -0.913764 \\
\hline 21 & 6 & 0 & 7.296651 & -3.331639 & -2.234449 \\
\hline 22 & 6 & 0 & 6.665981 & -2.624664 & -3.258646 \\
\hline 23 & 6 & 0 & 5.126367 & 2.342041 & -2.369951 \\
\hline 24 & 6 & 0 & 6.920654 & 1.310576 & -1.135255 \\
\hline 25 & 6 & 0 & 6.057261 & 3.042150 & -3.136720 \\
\hline 26 & 6 & 0 & 7.425377 & 2.878489 & -2.905410 \\
\hline 27 & 6 & 0 & 7.855421 & 2.011840 & -1.899971 \\
\hline 28 & 6 & 0 & 4.142063 & 1.339951 & 4.596233 \\
\hline 29 & 6 & 0 & 4.127195 & 2.209704 & 2.344782 \\
\hline 30 & 6 & 0 & 3.438623 & 1.696088 & 3.448003 \\
\hline 31 & 6 & 0 & 5.515112 & 2.387048 & 2.380645 \\
\hline 32 & 1 & 0 & 1.871644 & -7.222885 & 1.319783 \\
\hline 33 & 1 & 0 & 2.358260 & 1.609542 & 3.414508 \\
\hline 34 & 1 & 0 & 3.641118 & 0.949770 & 5.473601 \\
\hline 35 & 1 & 0 & 7.299884 & 2.147681 & 3.586243 \\
\hline 36 & 1 & 0 & 6.029144 & 2.819815 & 1.528724 \\
\hline 37 & 1 & 0 & 0.544300 & -7.674831 & 0.248710 \\
\hline 38 & 1 & 0 & -3.268026 & -1.215553 & 1.979187 \\
\hline 39 & 1 & 0 & 2.127588 & -7.264073 & -0.427376 \\
\hline 40 & 1 & 0 & 8.917123 & 1.881907 & -1.707219 \\
\hline 41 & 1 & 0 & 8.149708 & 3.429781 & -3.498975 \\
\hline
\end{tabular}




\begin{tabular}{|c|c|c|c|c|c|}
\hline 42 & 1 & 0 & 5.713856 & 3.726424 & -3.908072 \\
\hline 43 & 1 & 0 & 7.262503 & 0.630467 & -0.358393 \\
\hline 44 & 1 & 0 & 4.063667 & 2.499116 & -2.528466 \\
\hline 45 & 1 & 0 & 6.984513 & -2.755854 & -4.289191 \\
\hline 46 & 1 & 0 & 8.108558 & -4.016965 & -2.461546 \\
\hline 47 & 1 & 0 & 7.363142 & -3.698167 & -0.108793 \\
\hline 48 & 1 & 0 & 5.139162 & -1.193898 & -3.766064 \\
\hline 49 & 1 & 0 & 5.525883 & -2.145636 & 0.412388 \\
\hline 50 & 1 & 0 & 5.055544 & 0.301924 & 0.360500 \\
\hline 51 & 1 & 0 & 3.636550 & -0.233247 & -2.280741 \\
\hline 52 & 1 & 0 & 1.654006 & -1.877605 & -2.171450 \\
\hline 53 & 1 & 0 & 3.025242 & -2.953513 & -1.903010 \\
\hline 54 & 1 & 0 & 2.669973 & -4.954299 & -0.943060 \\
\hline 55 & 1 & 0 & -0.826748 & -5.788945 & 1.387478 \\
\hline 56 & 1 & 0 & -1.525179 & -2.458946 & 2.200549 \\
\hline 57 & 1 & 0 & -2.278108 & -4.049881 & 2.058936 \\
\hline 58 & 7 & 0 & 3.321861 & 1.387379 & -0.175161 \\
\hline 59 & 8 & 0 & 3.806814 & 3.908397 & 0.376506 \\
\hline 60 & 8 & 0 & 1.771417 & 2.632968 & 1.193330 \\
\hline 61 & 8 & 0 & -3.420425 & 0.490124 & -2.996778 \\
\hline 62 & 8 & 0 & -5.745651 & -0.053485 & -2.119371 \\
\hline 63 & 16 & 0 & 3.241659 & 2.627774 & 0.820646 \\
\hline 64 & 16 & 0 & -4.386112 & 0.469095 & -1.882871 \\
\hline 65 & 1 & 0 & -5.327494 & -1.523350 & -0.234778 \\
\hline 66 & 6 & 0 & -4.748297 & -2.753993 & 2.091727 \\
\hline 67 & 6 & 0 & -5.532360 & -3.731817 & 1.460512 \\
\hline 68 & 6 & 0 & -4.821715 & -2.636263 & 3.484635 \\
\hline 69 & 6 & 0 & -6.364776 & -4.568995 & 2.203351 \\
\hline 70 & 6 & 0 & -5.656864 & -3.469659 & 4.231667 \\
\hline 71 & 1 & 0 & -4.228059 & -1.876803 & 3.987833 \\
\hline 72 & 6 & 0 & -6.429639 & -4.439906 & 3.592868 \\
\hline 73 & 1 & 0 & -6.966466 & -5.318575 & 1.696374 \\
\hline 74 & 1 & 0 & -5.702383 & -3.359148 & 5.311734 \\
\hline 75 & 1 & 0 & -7.079842 & -5.090082 & 4.171706 \\
\hline 76 & 6 & 0 & -5.420235 & 0.075992 & 1.170824 \\
\hline 77 & 6 & 0 & -4.783412 & 1.074000 & 1.921785 \\
\hline 78 & 6 & 0 & -6.816286 & -0.006143 & 1.212955 \\
\hline 79 & 6 & 0 & -5.522915 & 1.961394 & 2.703160 \\
\hline 80 & 1 & 0 & -3.701462 & 1.170726 & 1.873472 \\
\hline
\end{tabular}




\begin{tabular}{|c|c|c|c|c|c|}
\hline 81 & 6 & 0 & -7.561179 & 0.880291 & 1.994369 \\
\hline 82 & 1 & 0 & -7.322266 & -0.764543 & 0.621710 \\
\hline 83 & 6 & 0 & -6.916630 & 1.865355 & 2.743864 \\
\hline 84 & 1 & 0 & -5.013325 & 2.732645 & 3.275098 \\
\hline 85 & 1 & 0 & -8.645124 & 0.802257 & 2.013062 \\
\hline 86 & 1 & 0 & -7.494288 & 2.558233 & 3.349840 \\
\hline 87 & 6 & 0 & -4.589003 & 2.210594 & -1.424435 \\
\hline 88 & 6 & 0 & -5.868737 & 2.740265 & -1.261852 \\
\hline 89 & 6 & 0 & -3.449786 & 3.008737 & -1.269264 \\
\hline 90 & 6 & 0 & -6.018076 & 4.084533 & -0.923403 \\
\hline 91 & 1 & 0 & -6.731890 & 2.099642 & -1.398688 \\
\hline 92 & 6 & 0 & -3.589549 & 4.350138 & -0.931418 \\
\hline 93 & 1 & 0 & -2.466429 & 2.576526 & -1.424360 \\
\hline 94 & 6 & 0 & -4.876470 & 4.865570 & -0.761294 \\
\hline 95 & 1 & 0 & -6.995262 & 4.530305 & -0.785681 \\
\hline 96 & 1 & 0 & -2.732633 & 5.000184 & -0.803212 \\
\hline 97 & 7 & 0 & -2.774298 & -2.616713 & 0.549619 \\
\hline 98 & 1 & 0 & -3.212829 & -3.354629 & -0.004286 \\
\hline 99 & 8 & 0 & -0.125778 & -1.511561 & 0.087910 \\
\hline 100 & 7 & 0 & -3.668218 & -0.268399 & -0.606380 \\
\hline 101 & 1 & 0 & -5.504617 & -3.839461 & 0.377806 \\
\hline 102 & 8 & 0 & -1.668533 & -2.315239 & -2.577441 \\
\hline 103 & 6 & 0 & -1.285455 & -1.939098 & -3.729026 \\
\hline 104 & 6 & 0 & -1.495620 & -2.945966 & -4.840161 \\
\hline 105 & 1 & 0 & -0.941140 & -2.696305 & -5.748280 \\
\hline 106 & 1 & 0 & -2.565001 & -2.981083 & -5.083699 \\
\hline 107 & 1 & 0 & -1.206959 & -3.942417 & -4.491716 \\
\hline 108 & 6 & 0 & -0.122143 & 1.737350 & -3.811282 \\
\hline 109 & 1 & 0 & -0.976336 & 2.421158 & -3.730590 \\
\hline 110 & 1 & 0 & 0.158470 & 1.659112 & -4.864322 \\
\hline 111 & 1 & 0 & 0.709879 & 2.186334 & -3.253522 \\
\hline 112 & 6 & 0 & -0.528068 & 0.408507 & -3.211870 \\
\hline 113 & 8 & 0 & -0.668820 & 0.397052 & -1.923502 \\
\hline 114 & 7 & 0 & 2.911127 & -1.247657 & -0.614613 \\
\hline 115 & 1 & 0 & 3.270096 & -1.655271 & 0.250980 \\
\hline 116 & 6 & 0 & -0.725350 & -0.680250 & -4.061089 \\
\hline 117 & 1 & 0 & -0.522715 & -0.513202 & -5.112700 \\
\hline 118 & 30 & 0 & -1.919109 & 1.201054 & -0.954770 \\
\hline 119 & 38 & 0 & 0.990375 & 0.576952 & -0.112420 \\
\hline
\end{tabular}




$\begin{array}{rrrrrr}120 & 7 & 0 & 6.275390 & 1.114852 & 5.818303 \\ 121 & 8 & 0 & 5.632991 & 0.661068 & 6.764114 \\ 122 & 8 & 0 & 7.495994 & 1.264164 & 5.803667 \\ 123 & 7 & 0 & -5.030319 & 6.284125 & -0.401168 \\ 124 & 8 & 0 & -4.005988 & 6.953149 & -0.265580 \\ 125 & 8 & 0 & -6.173299 & 6.716811 & -0.255746\end{array}$

The energies for the structure(a.u.):

Zero-point correction $=0.964589$ (Hartree/Particle)

Thermal correction to Energy $=1.035073$

Thermal correction to Enthalpy $=1.036017$

Thermal correction to Gibbs Free Energy $=0.843478$

Sum of electronic and zero-point Energies $=-4296.989367$

Sum of electronic and thermal Energies $=-4296.918883$

Sum of electronic and thermal Enthalpies $=-4296.917939$

Sum of electronic and thermal Free Energies $=-4297.110477$ 


\section{Crystal data and structure refinement}

Preparation of the single crystals of enantiopure $4 \mathrm{fs}$ : Compound $\mathbf{4 f s}(15.0 \mathrm{mg}$, $96 \%$ ee) was dissolved in the mixture of acetone and toluene $(v / v 1 / 3,2.0 \mathrm{~mL})$. The tube was sealed by a piece of weighing paper with several tiny holes, thus allowing slow evaporation of the solvents at room temperature. After $48 \mathrm{~h}$, several small particles could be observed at the bottom of the tube. The crystals were chosen and subjected to the single crystal X-ray diffraction analysis for the determination of the absolute configuration of $4 \mathrm{fs}$. The data were collected by a Rigaku Gemini equipped with a $\mathrm{Cu}$ radiation source $(\mathrm{K} \alpha=1.54184 \AA$ ) at 293(2) K. CCDC 2048629 (4fs) contains the supplementary crystallographic data for this paper. These data can be obtained free of charge via www.ccdc.cam.ac.uk/data_request/cif.

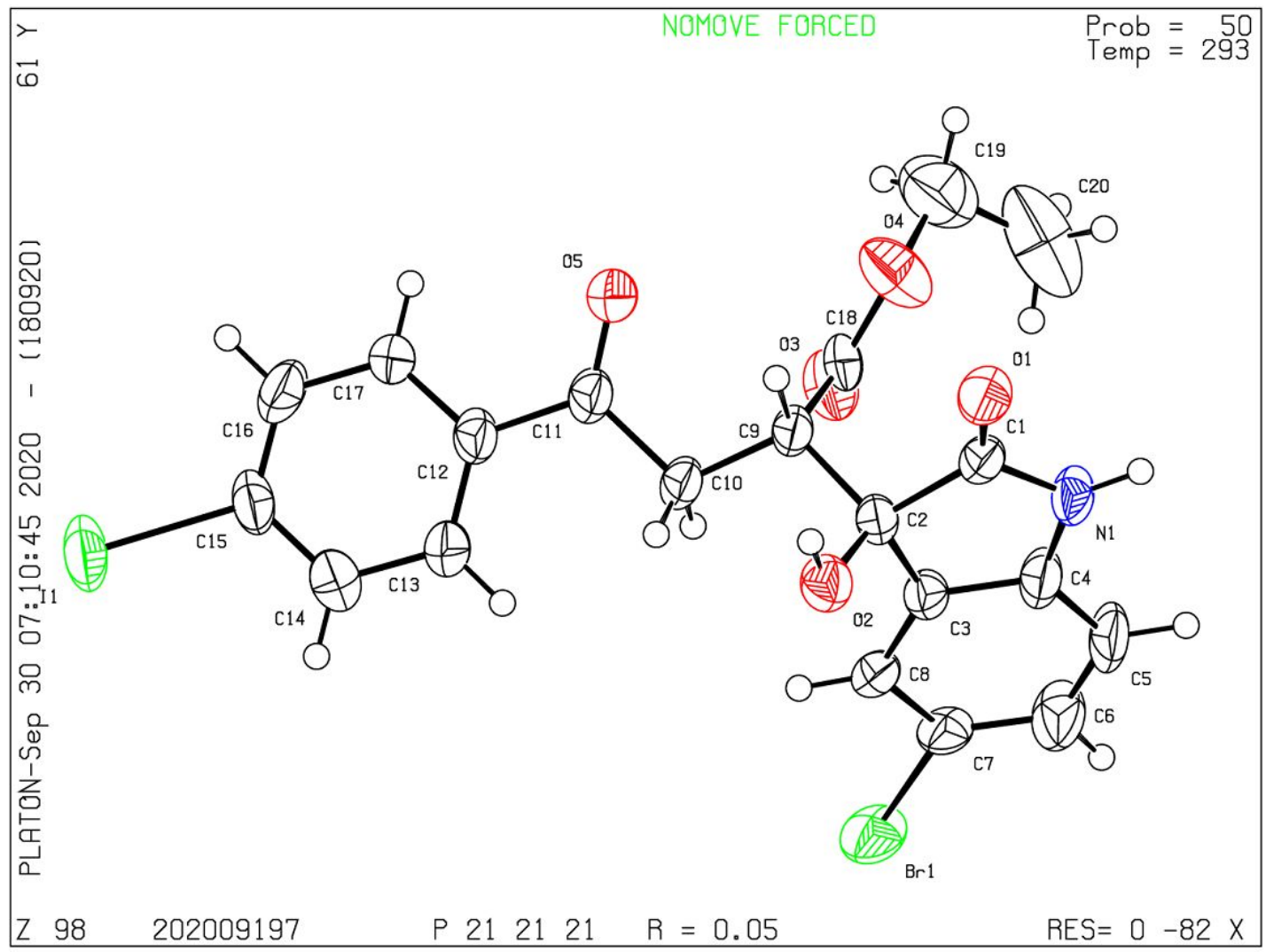

(ellipsoid contour probability 30\%)

Table S5. Crystal data and structure refinement for 202009197 (4fs).

Identification code

Empirical formula

Formula weight

Temperature/K

Crystal system

Space group
202009197

$\mathrm{C}_{20} \mathrm{H}_{17} \mathrm{BrINO}_{5}$

558.16

293(2)

orthorhombic

$\mathrm{P} 22_{1} 2_{1}$ 


\begin{tabular}{|c|c|}
\hline $\mathrm{a} / \AA$ & $8.1150(6)$ \\
\hline $\mathrm{b} / \AA$ & $15.6444(6)$ \\
\hline $\mathrm{c} / \AA$ & $15.9992(5)$ \\
\hline$\alpha /^{\circ}$ & 90 \\
\hline$\beta /^{\circ}$ & 90 \\
\hline$\gamma /{ }^{\circ}$ & 90 \\
\hline Volume $/ \AA^{3}$ & $2031.17(18)$ \\
\hline $\mathrm{Z}$ & 4 \\
\hline$\rho_{\text {calc }} \mathrm{g} / \mathrm{cm}^{3}$ & 1.825 \\
\hline$\mu / \mathrm{mm}^{-1}$ & 14.967 \\
\hline $\mathrm{F}(000)$ & 1088.0 \\
\hline Crystal size $/ \mathrm{mm}^{3}$ & $0.15 \times 0.09 \times 0.07$ \\
\hline Radiation & $\operatorname{CuK} \alpha(\lambda=1.54184)$ \\
\hline $2 \Theta$ range for data collection $/{ }^{\circ}$ & 7.904 to 134.274 \\
\hline Index ranges & $-9 \leq \mathrm{h} \leq 8,-18 \leq \mathrm{k} \leq 18,-15 \leq 1 \leq 19$ \\
\hline Reflections collected & 7369 \\
\hline Independent reflections & $3560\left[\mathrm{R}_{\text {int }}=0.0413, \mathrm{R}_{\text {sigma }}=0.0579\right]$ \\
\hline Data/restraints/parameters & $3560 / 1 / 255$ \\
\hline Goodness-of-fit on $\mathrm{F}^{2}$ & 1.035 \\
\hline Final $R$ indexes $[\mathrm{I}>=2 \sigma(\mathrm{I})]$ & $\mathrm{R}_{1}=0.0454, \mathrm{wR}_{2}=0.1024$ \\
\hline Final $\mathrm{R}$ indexes [all data] & $\mathrm{R}_{1}=0.0566, \mathrm{wR}_{2}=0.1097$ \\
\hline Largest diff. peak/hole / e $\AA^{-3}$ & $0.81 /-0.62$ \\
\hline Flack parameter & $-0.009(7)$ \\
\hline
\end{tabular}




\section{Copies of HPLC}

(1) 3aa
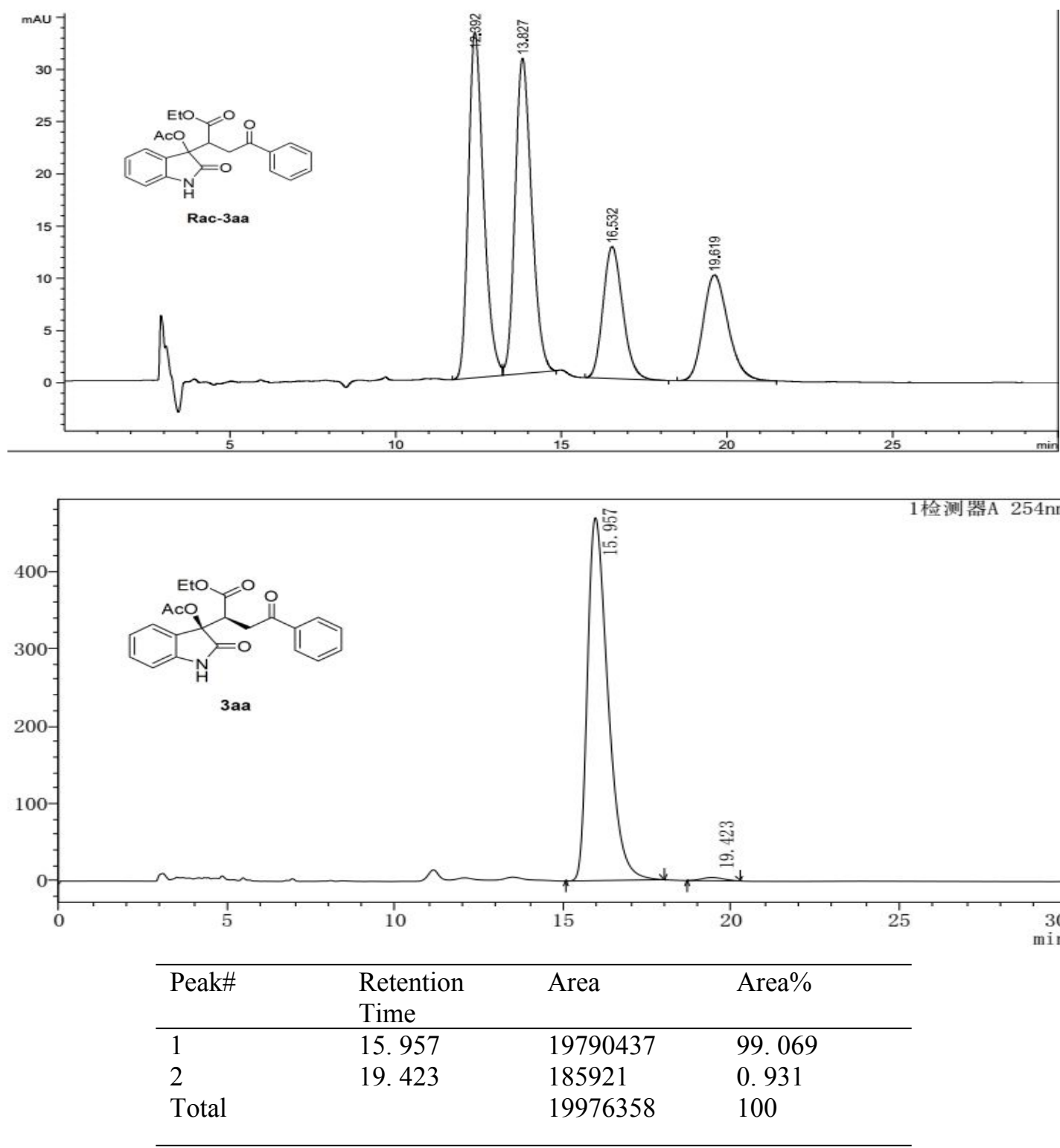
(2) $3 a b$
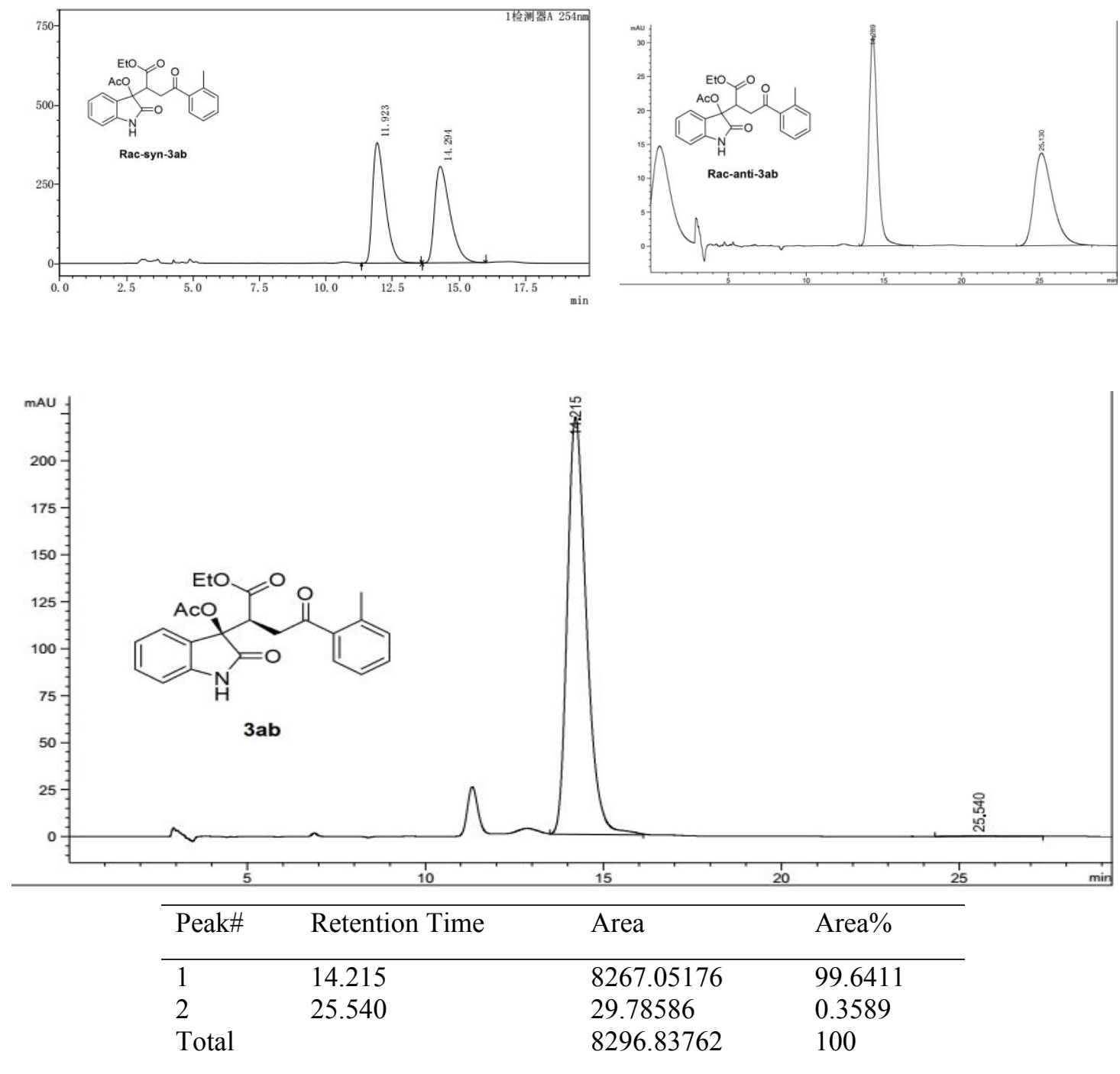
(3) $3 \mathrm{ac}$
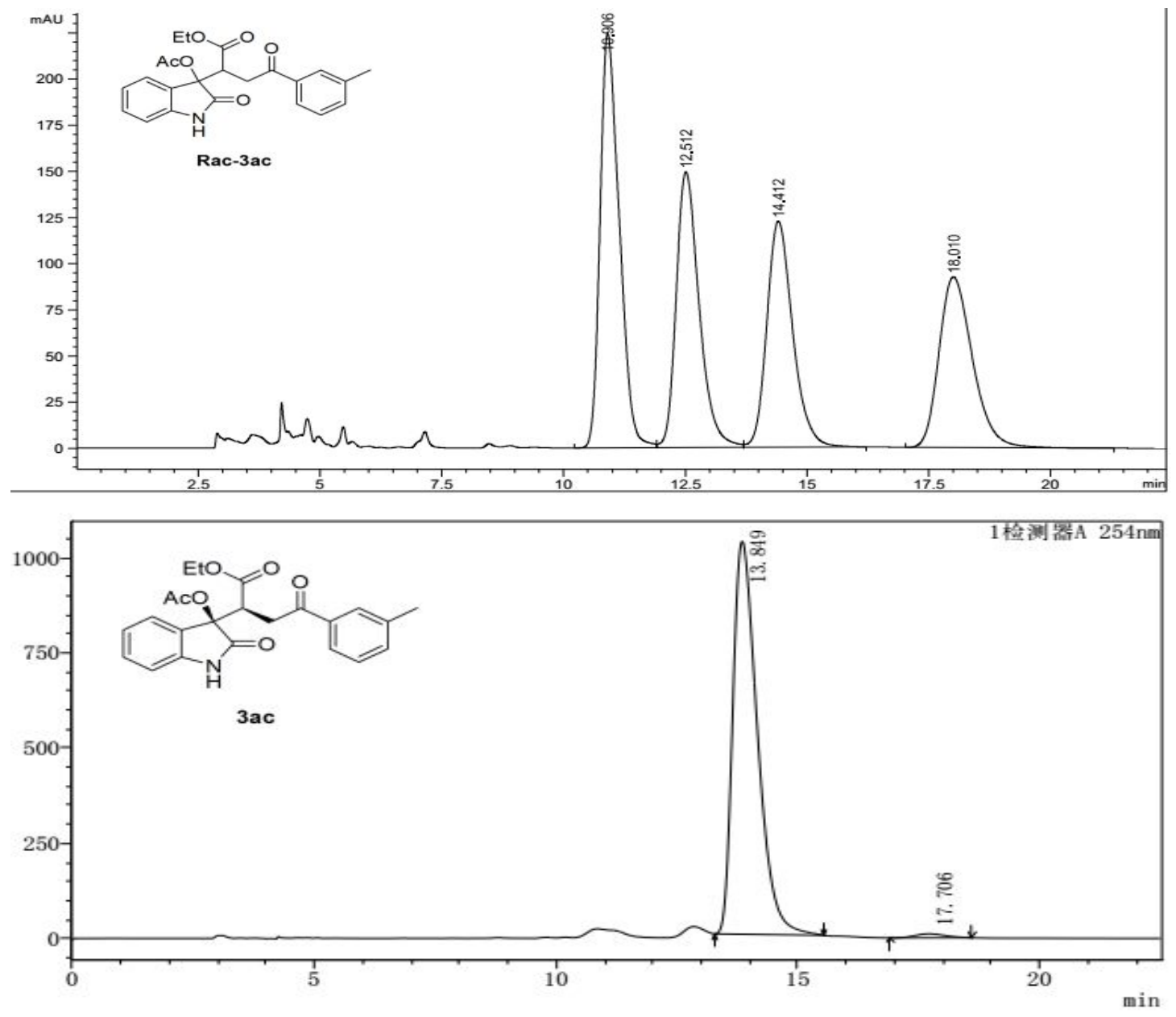

\begin{tabular}{llll}
\hline Peak\# & Retention Time & Area & Area\% \\
\hline 1 & 13.849 & 36432428 & 98.748 \\
2 & 17.706 & 462013 & 1.252 \\
Total & & 36894441 & 100 \\
\hline
\end{tabular}


(4) 3ad
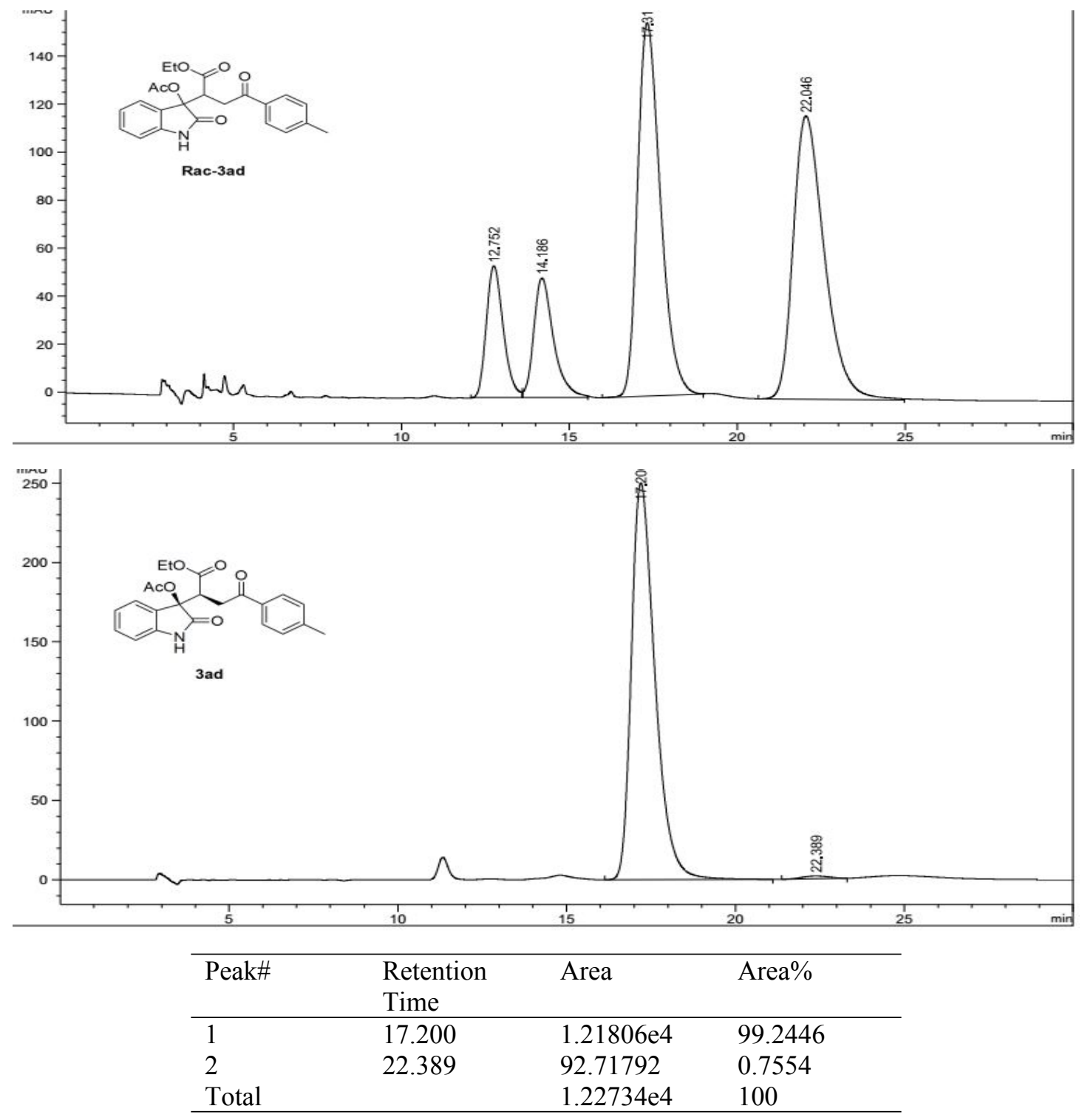
(5) $3 \mathrm{ae}$

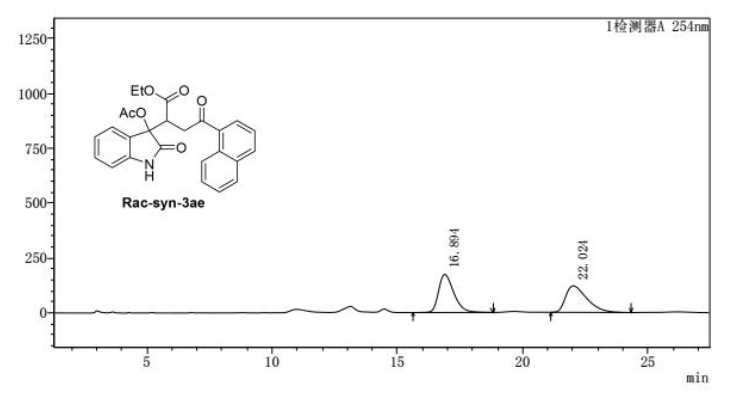

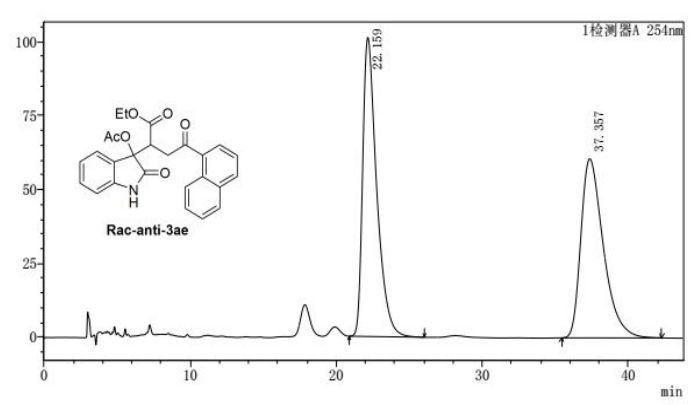

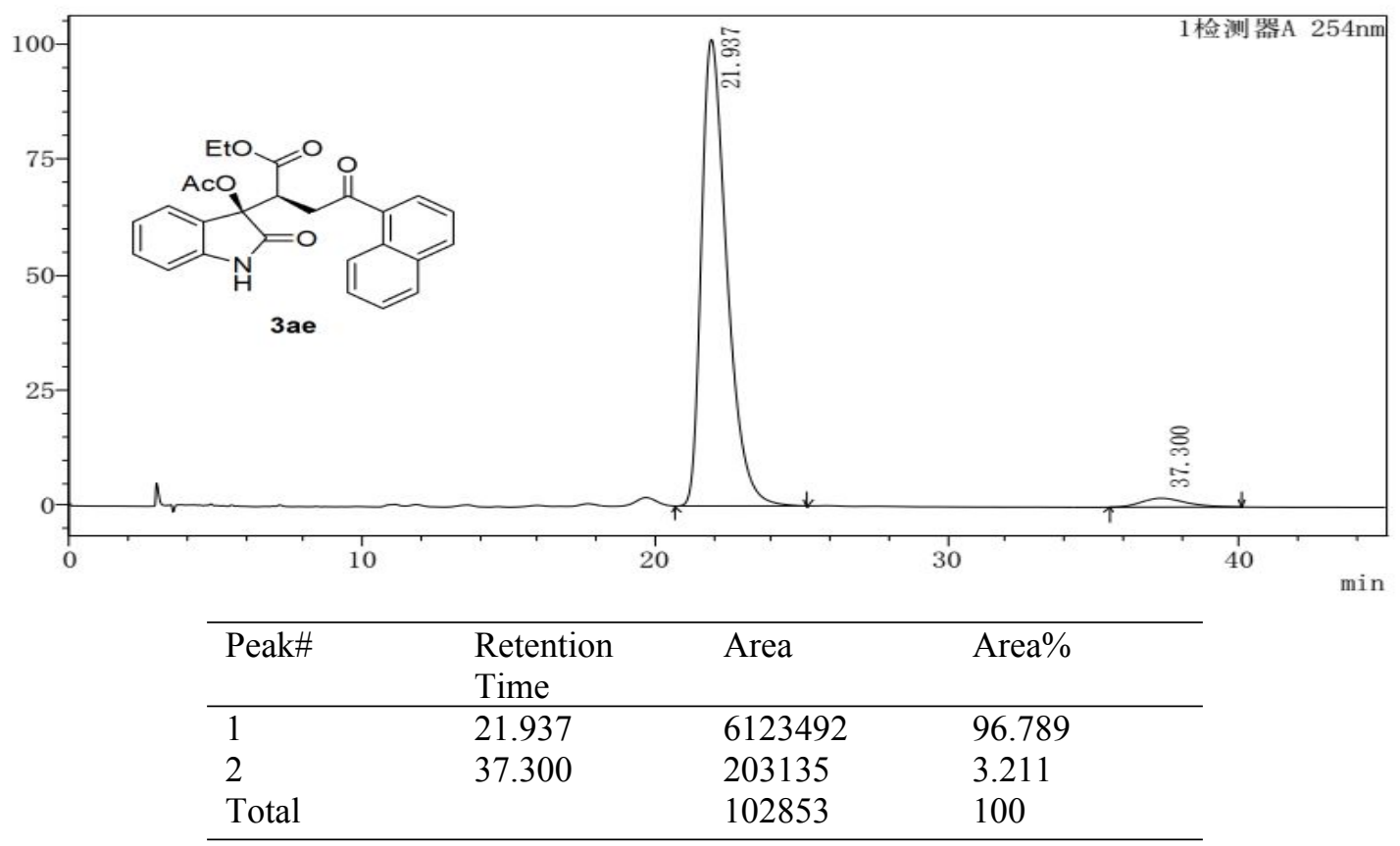


(6) $3 \mathrm{af}$
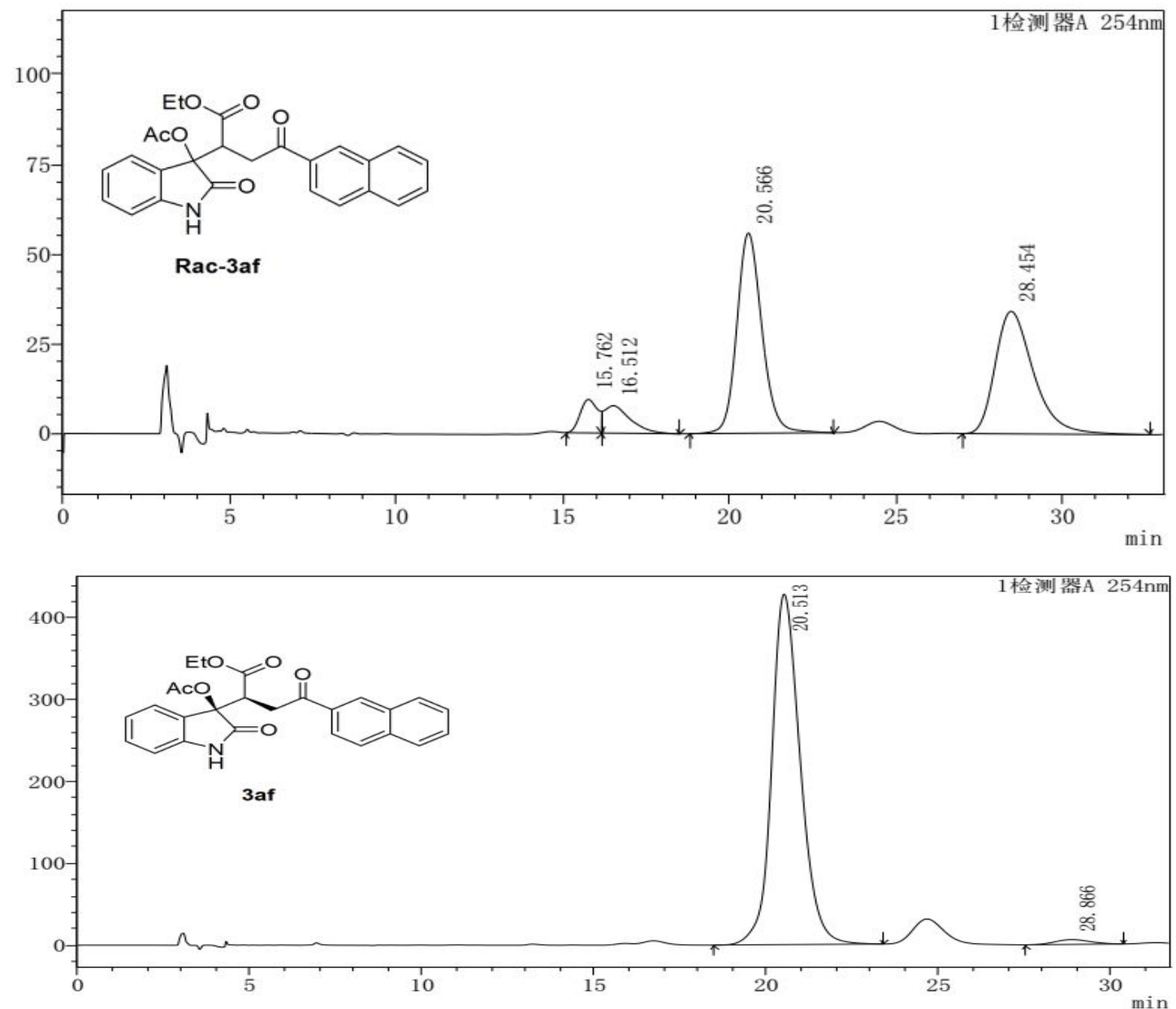

\begin{tabular}{llll}
\hline Peak\# & $\begin{array}{l}\text { Retention } \\
\text { Time }\end{array}$ & Area & Area\% \\
\hline 1 & 20.513 & 24685279 & 98.334 \\
2 & 28.866 & 418308 & 1.666 \\
Total & & 25103587 & 100 \\
\hline
\end{tabular}


(7) $3 \mathrm{ag}$
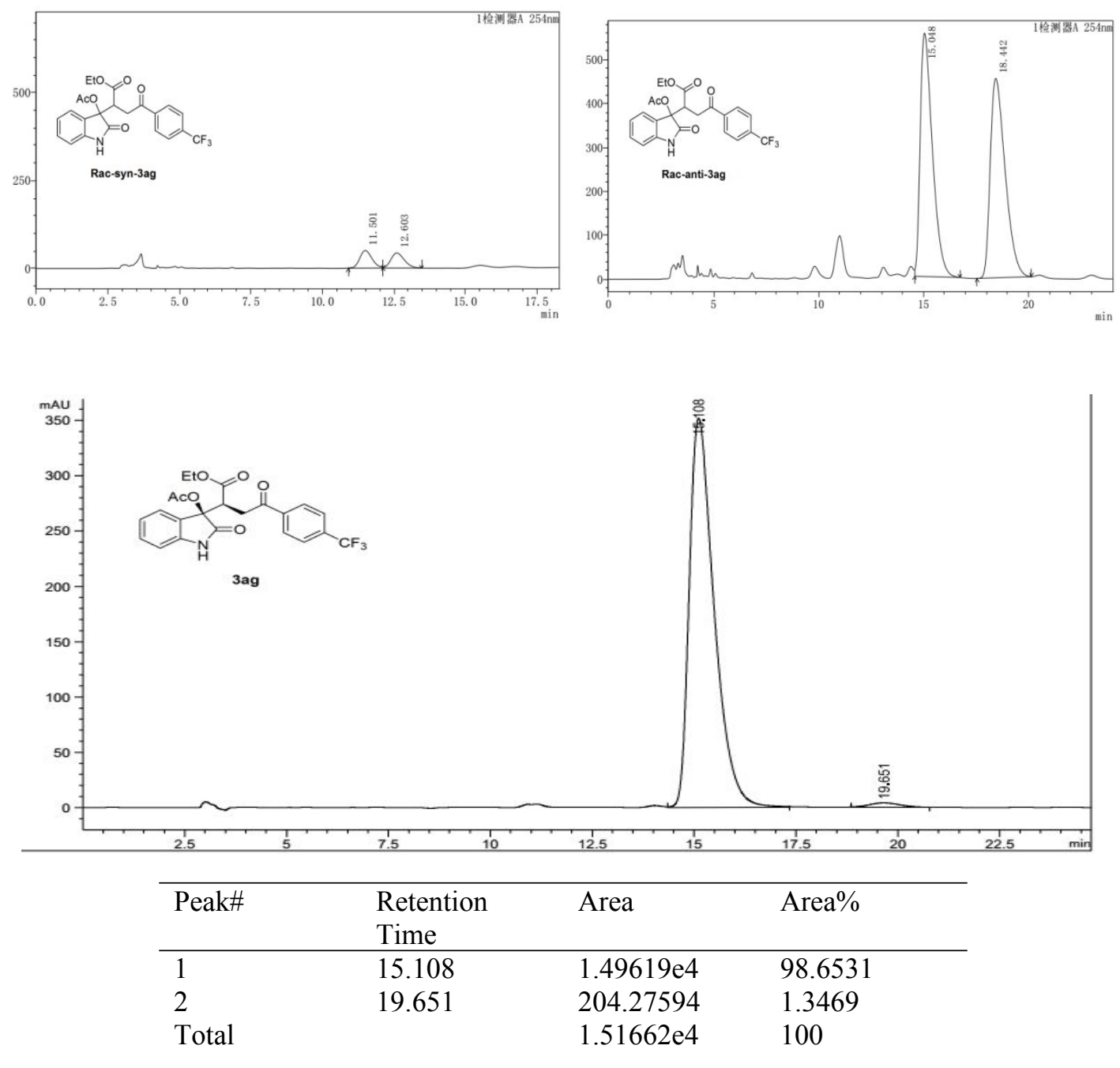
(8) 3ah
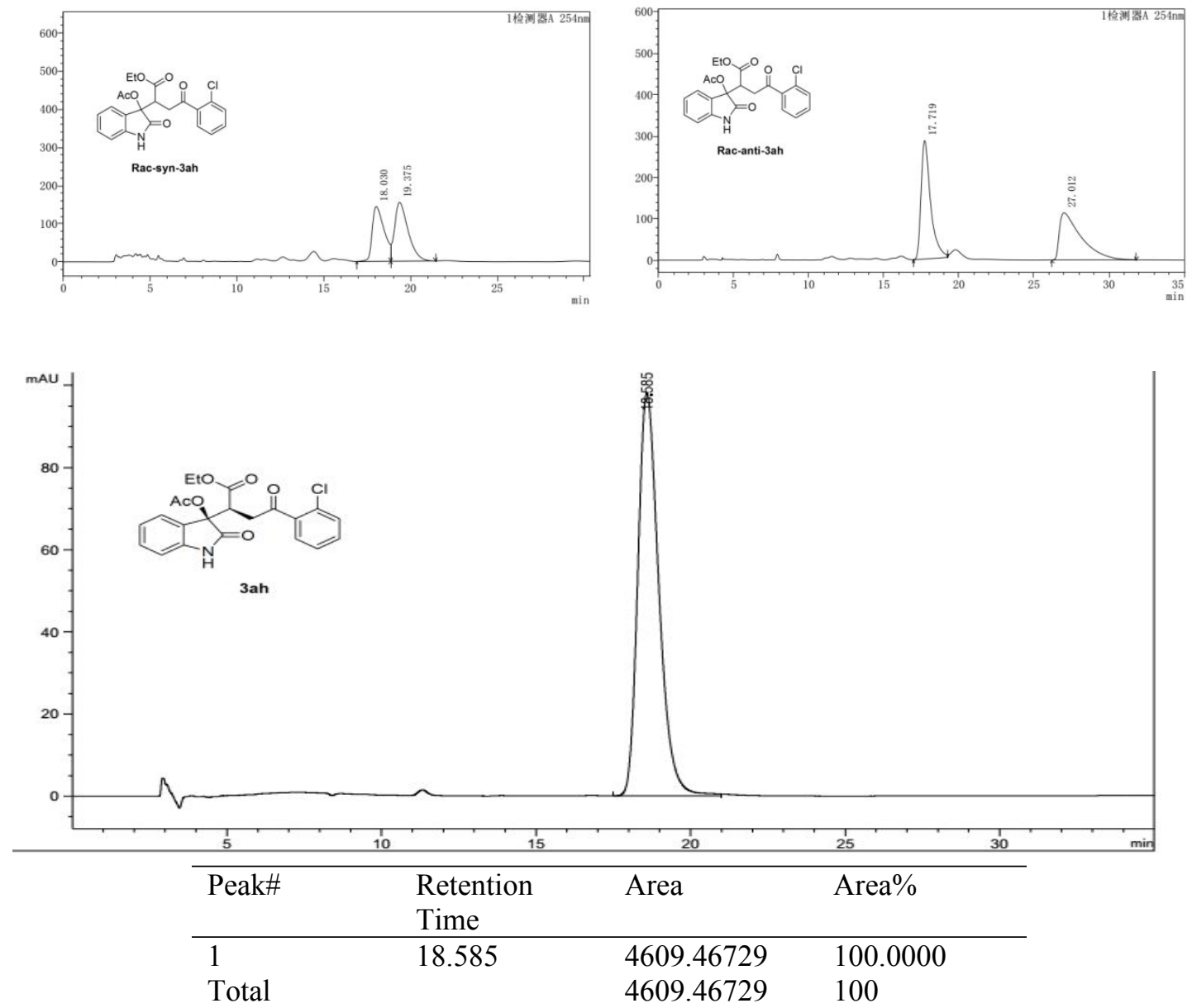
(9) $3 \mathbf{a i}$

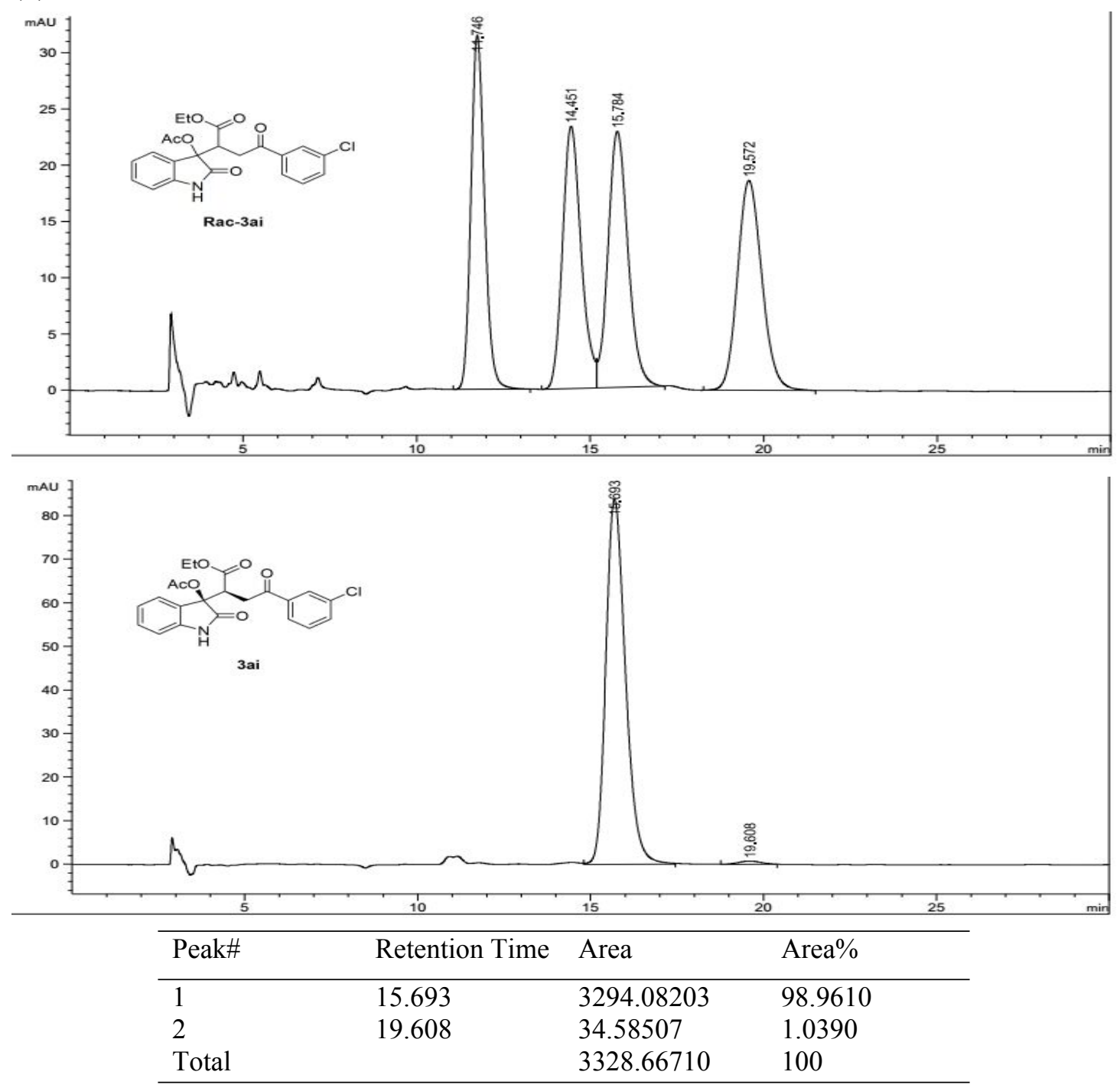


(10) 3aj

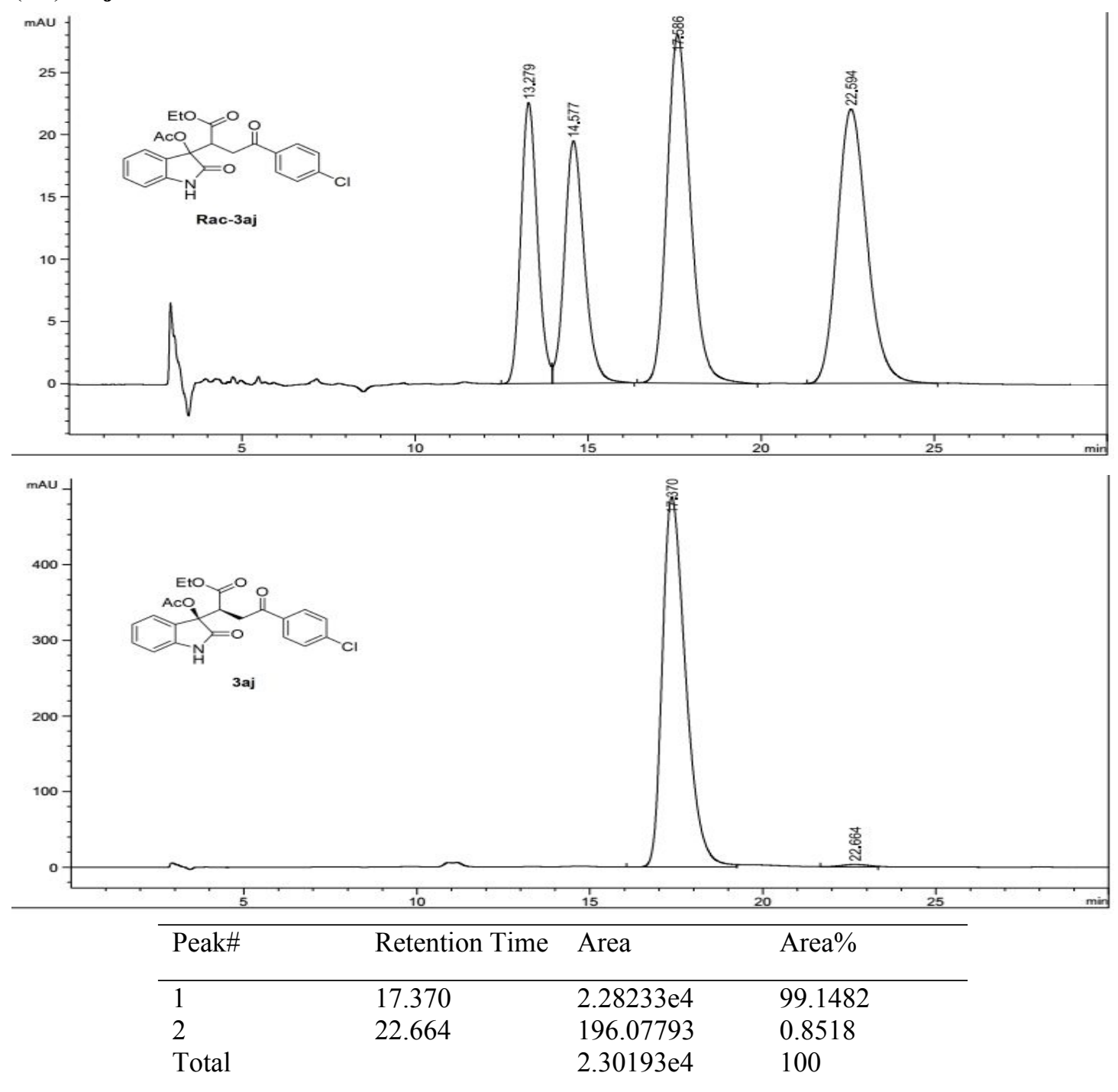


(11) 3ak

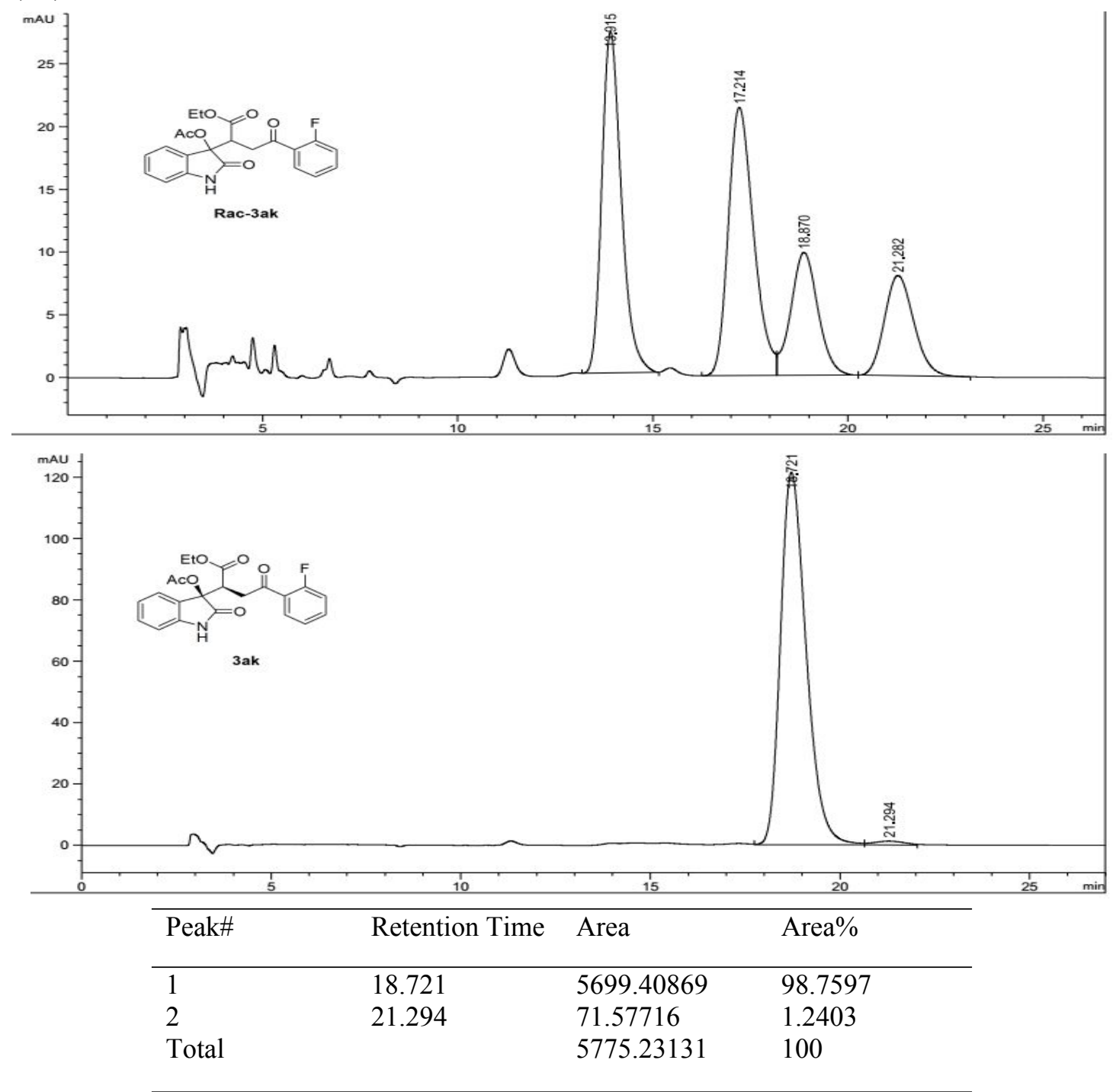


(12) 3al

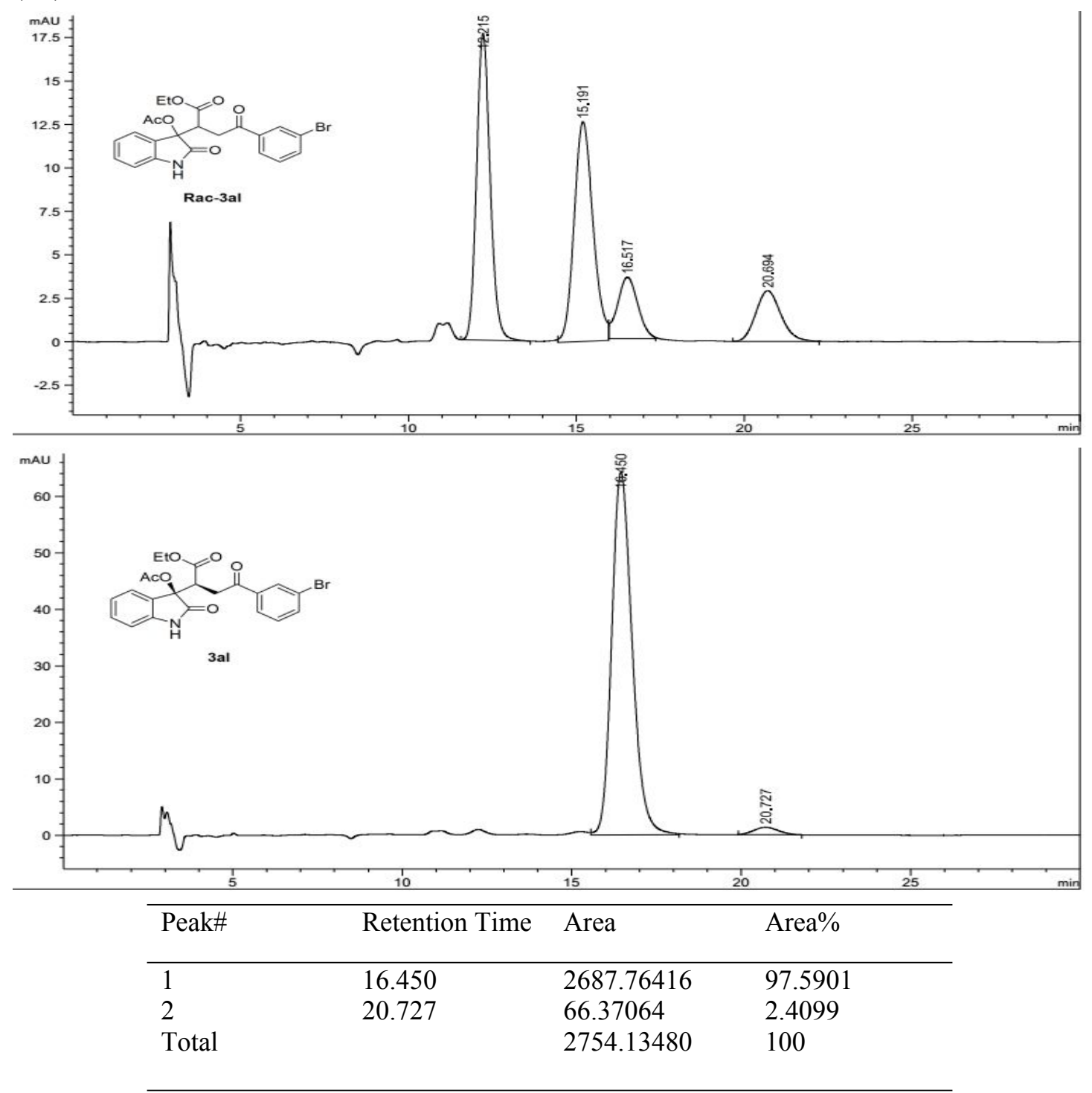


(13) 3am

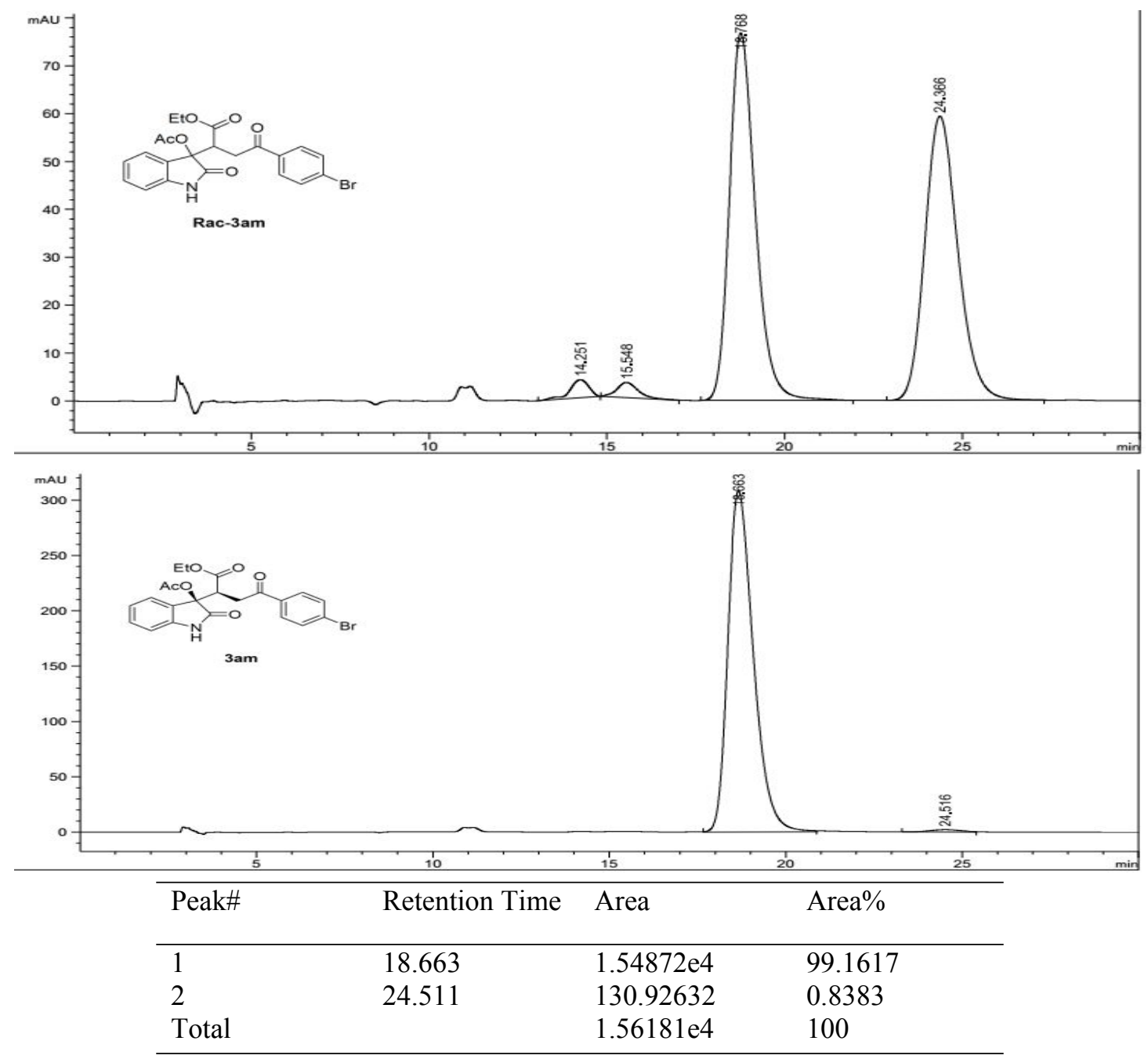


(14) 3an
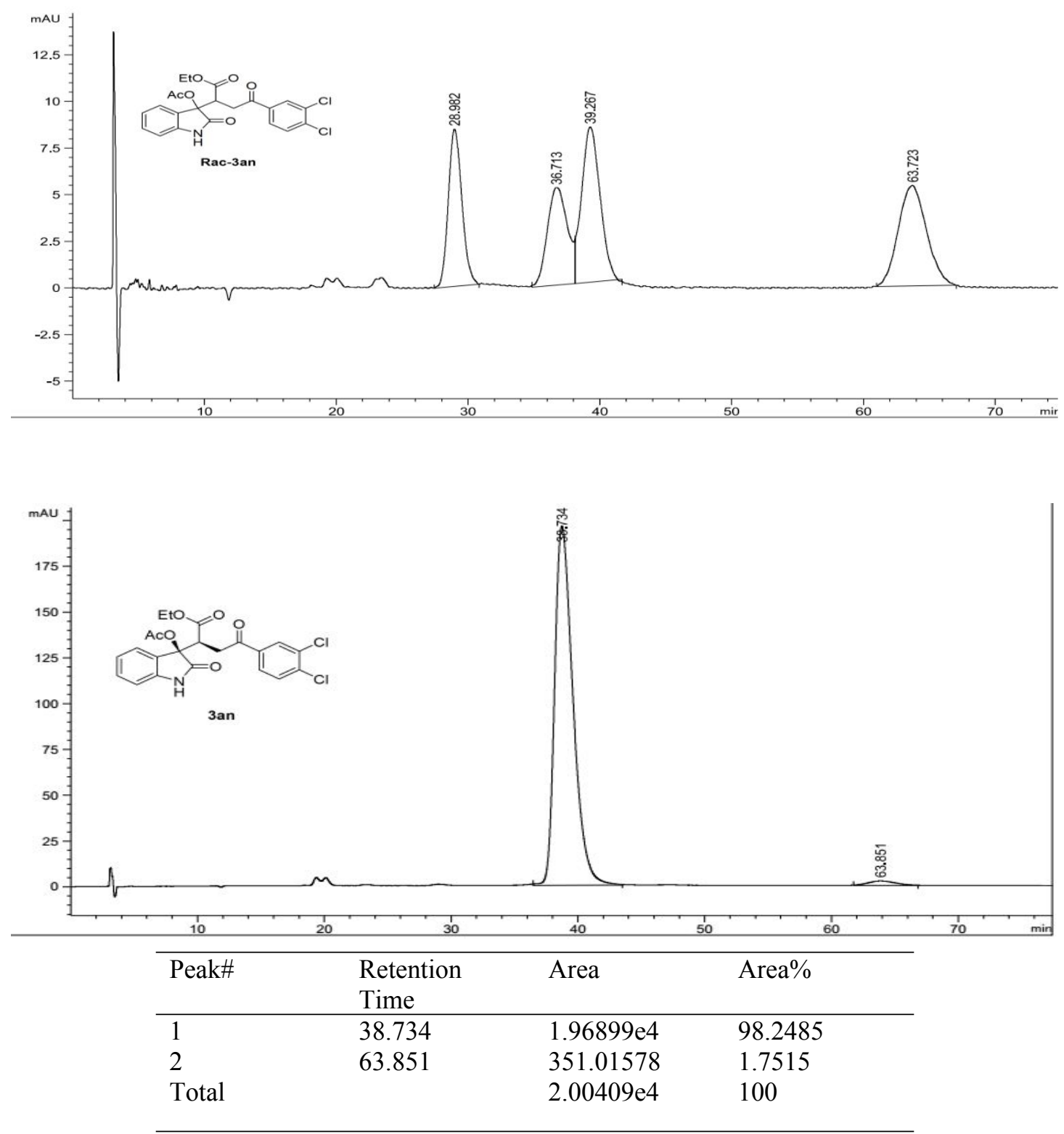
(15) $3 \mathrm{ao}$

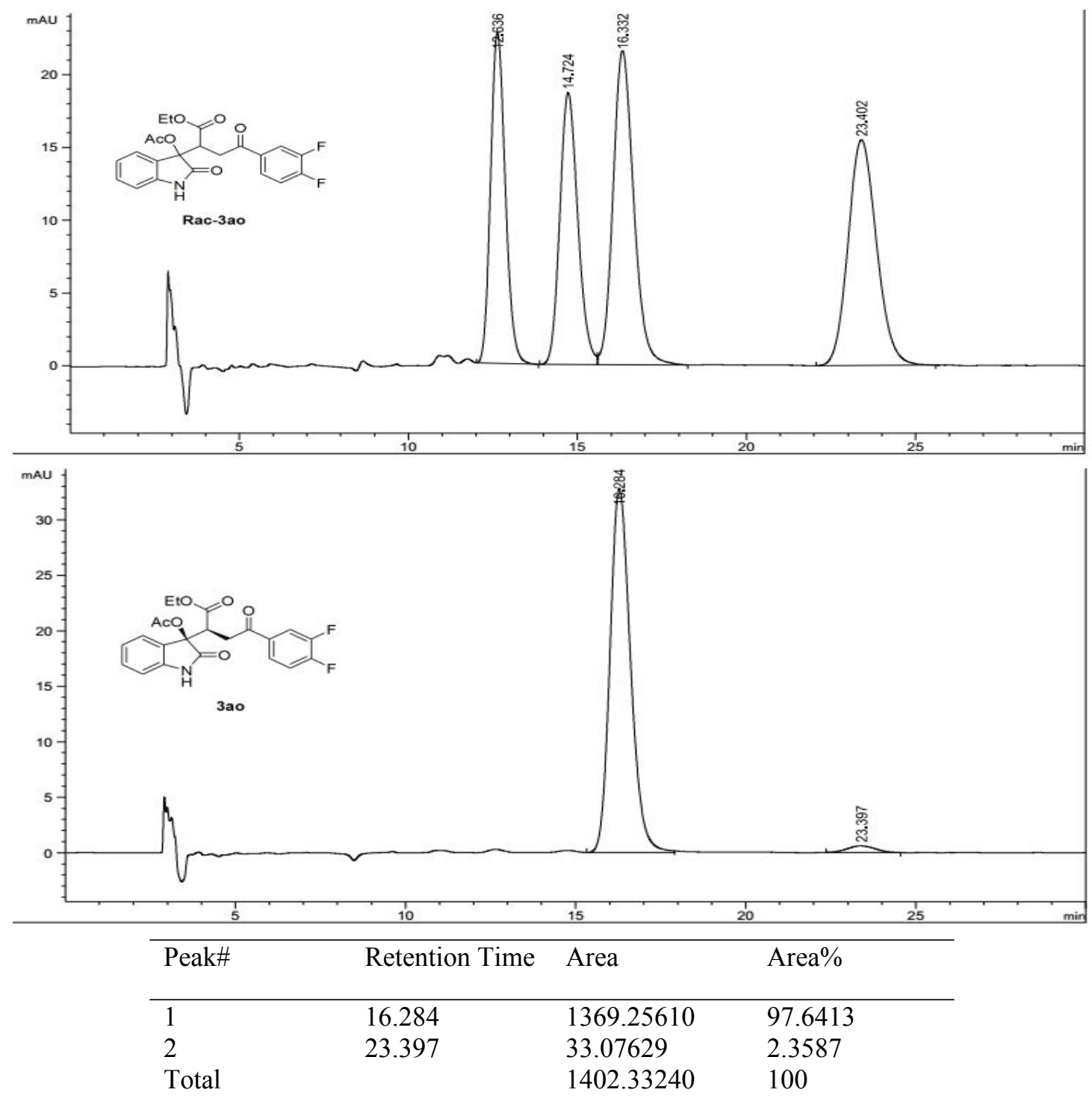


(16) 3ap
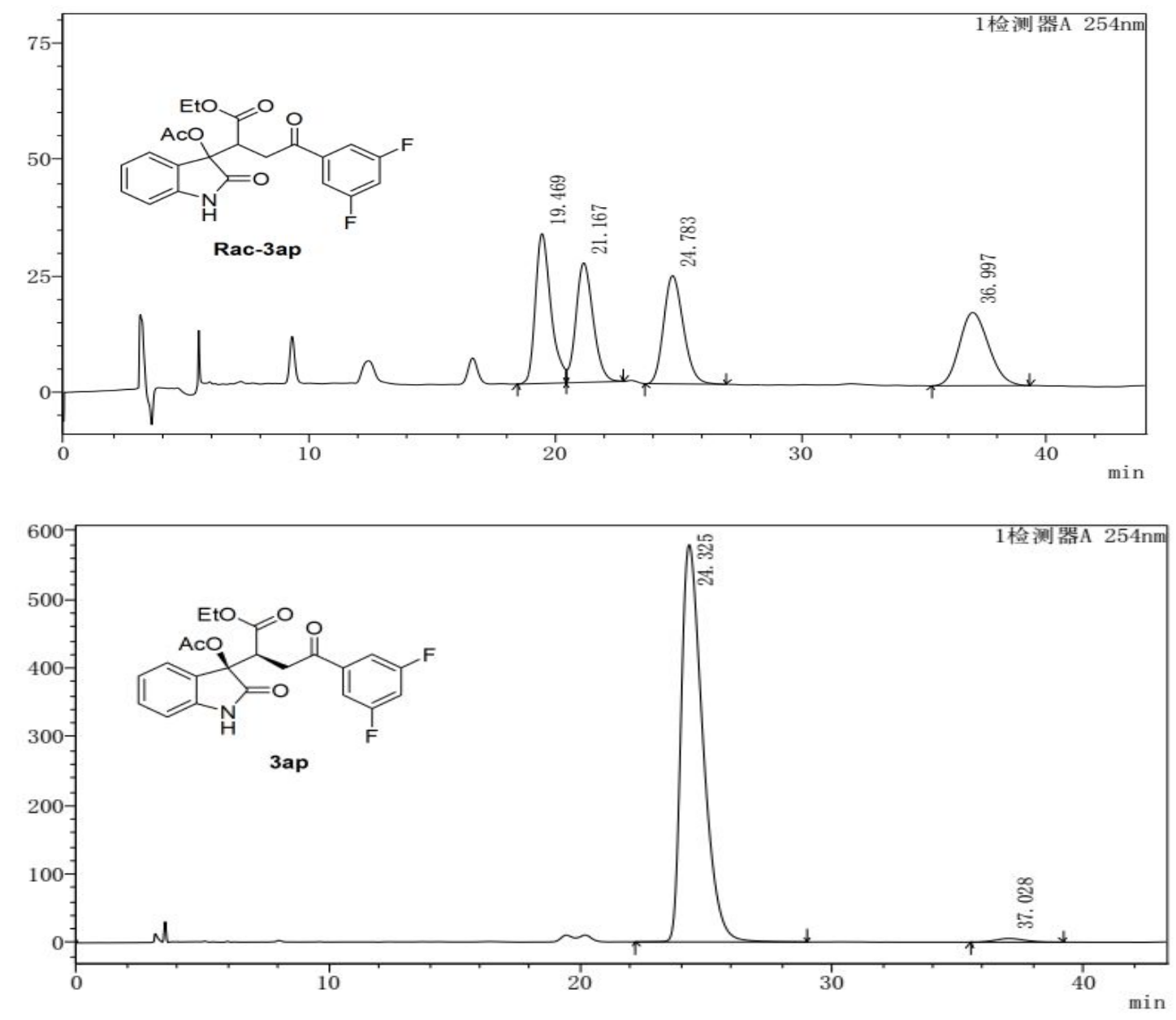

\begin{tabular}{llll}
\hline Peak\# & Retention Time & Area & Area\% \\
\hline 1 & 24.325 & 34391101 & 98.754 \\
2 & 37.028 & 433810 & 1.246 \\
Total & & 34824911 & 100 \\
\hline
\end{tabular}


(17) 3aq

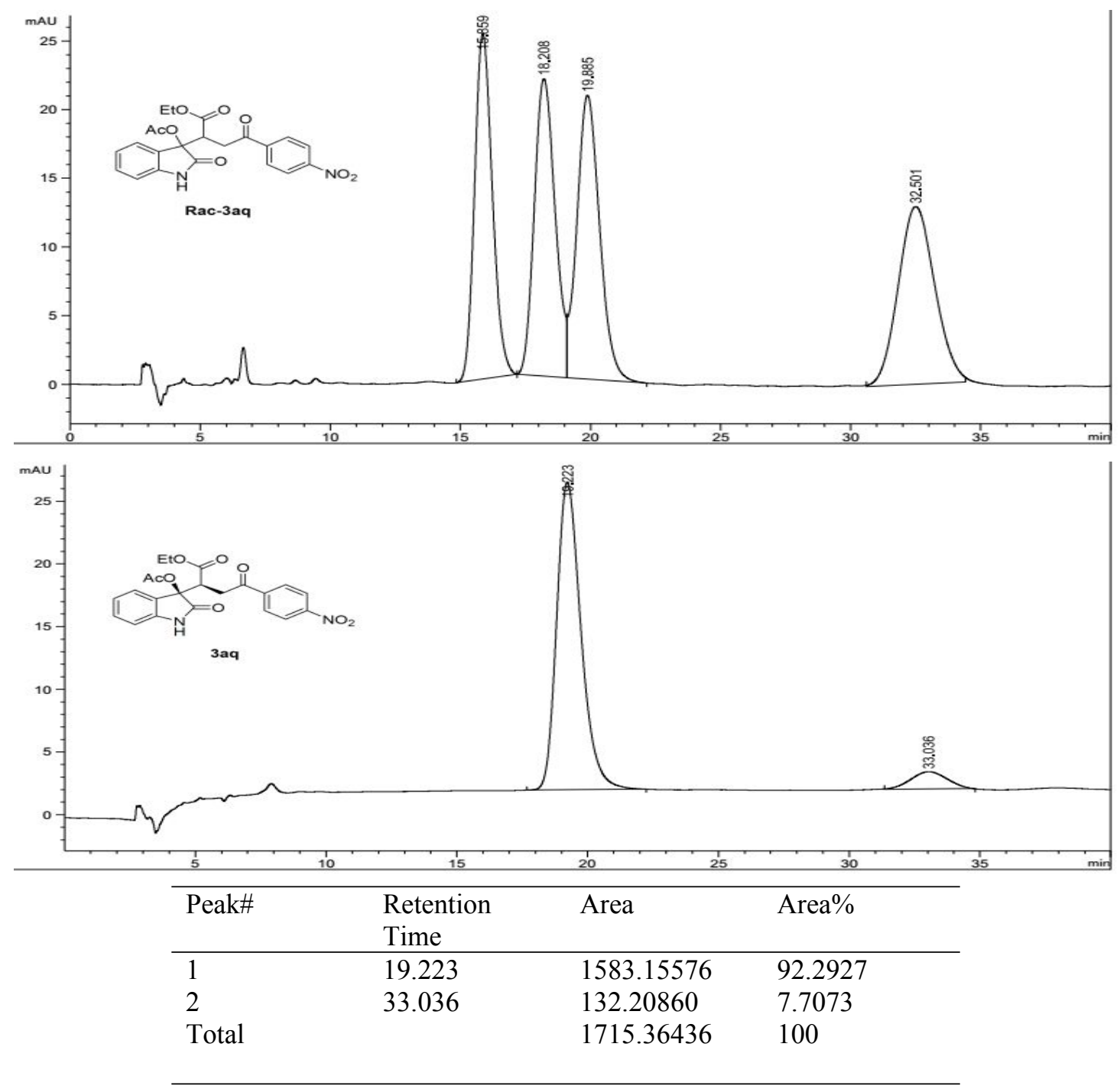


(18) 3ar
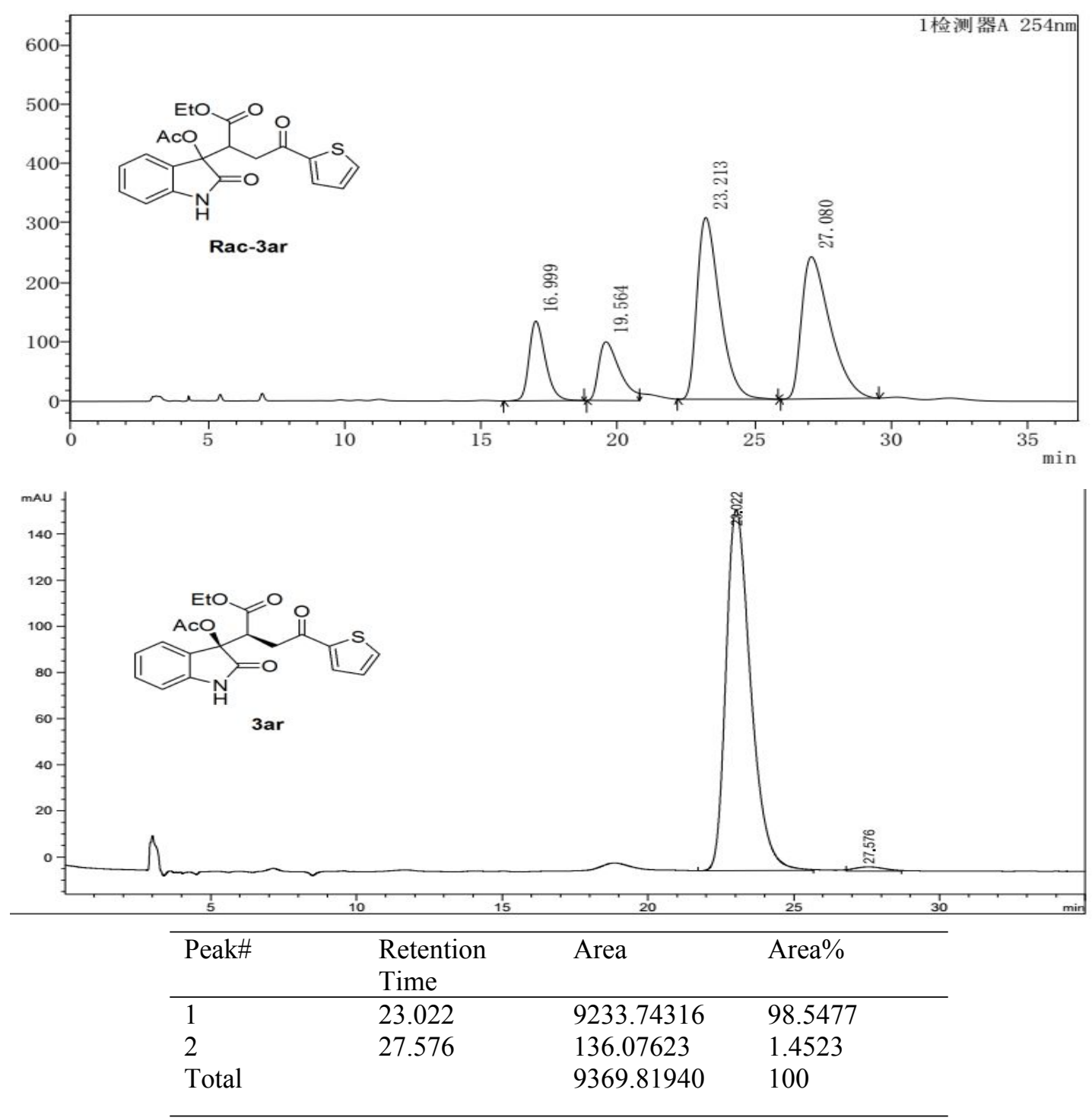
(19) $3 \mathbf{b a}$
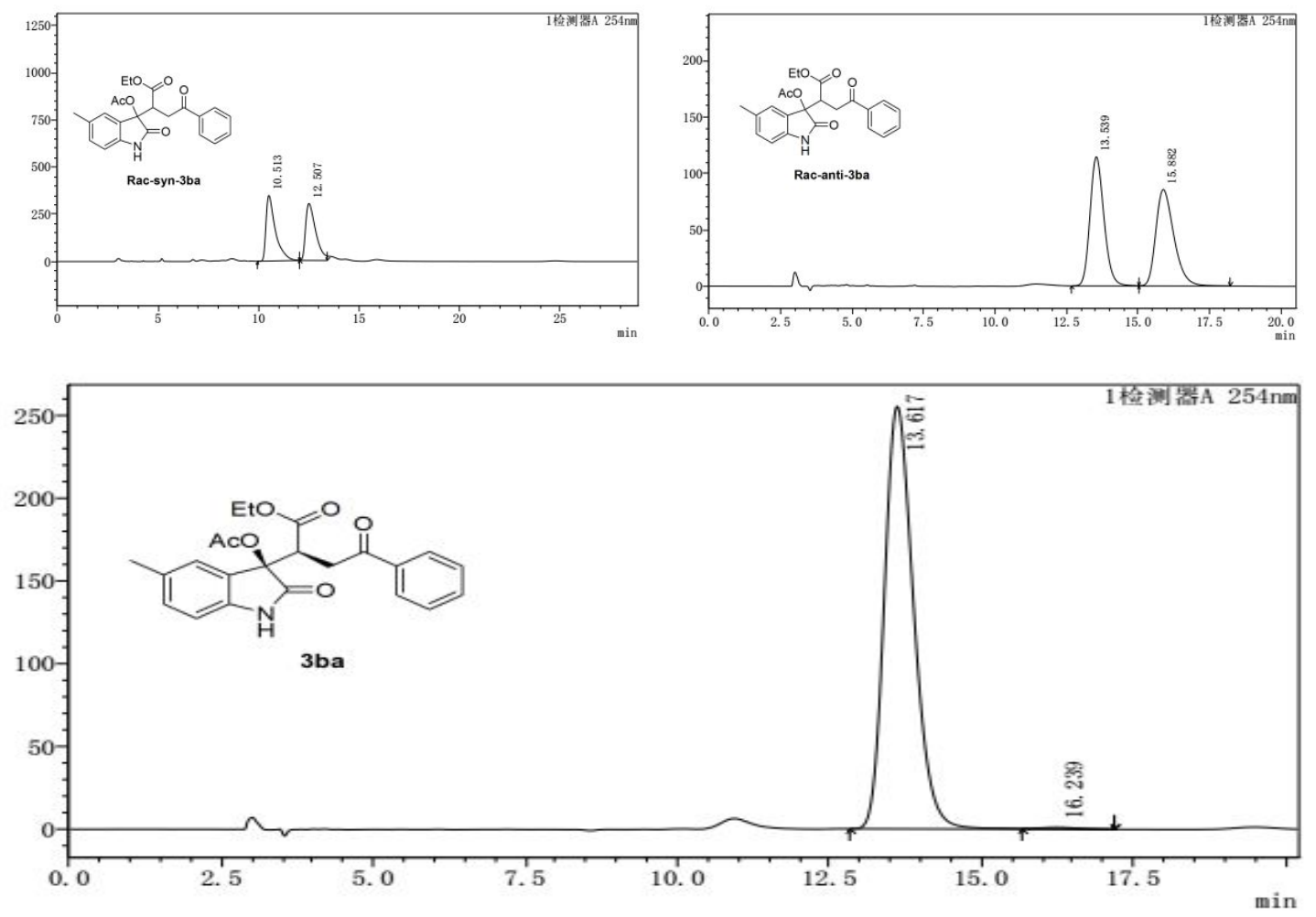

\begin{tabular}{llll}
\hline Peak\# & $\begin{array}{l}\text { Retention } \\
\text { Time }\end{array}$ & Area & Area\% \\
\hline 1 & 13.617 & 8545009 & 99.612 \\
2 & 16.239 & 33294 & 0.388 \\
Total & & 8578303 & 100 \\
\hline
\end{tabular}


(20) 3 ca
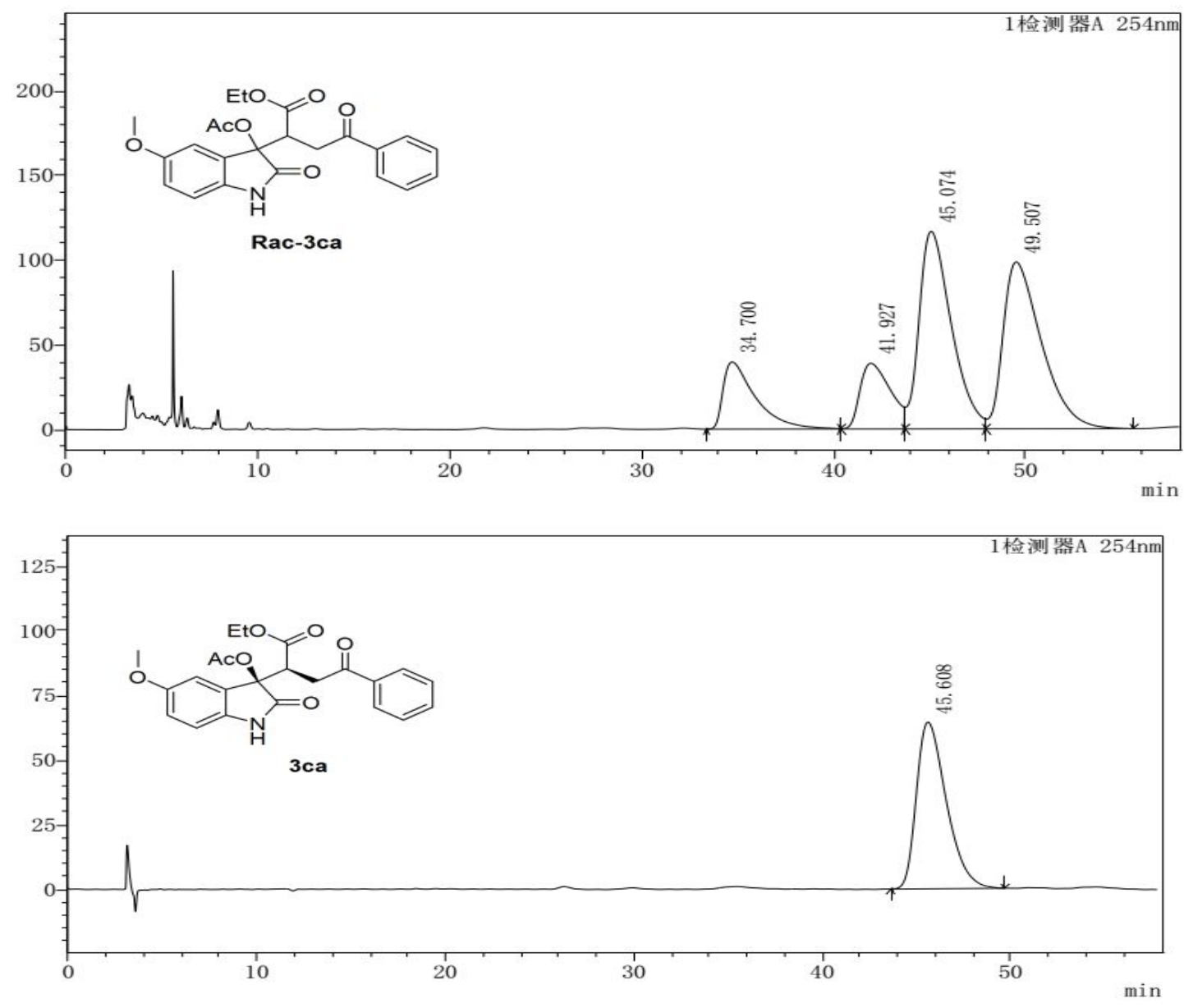

\begin{tabular}{llll}
\hline Peak\# & $\begin{array}{l}\text { Retention } \\
\text { Time }\end{array}$ & Area & Area\% \\
\hline 1 & 45.608 & 7117705 & 100.000 \\
Total & & 7117705 & 100 \\
\hline
\end{tabular}


(21) 3da

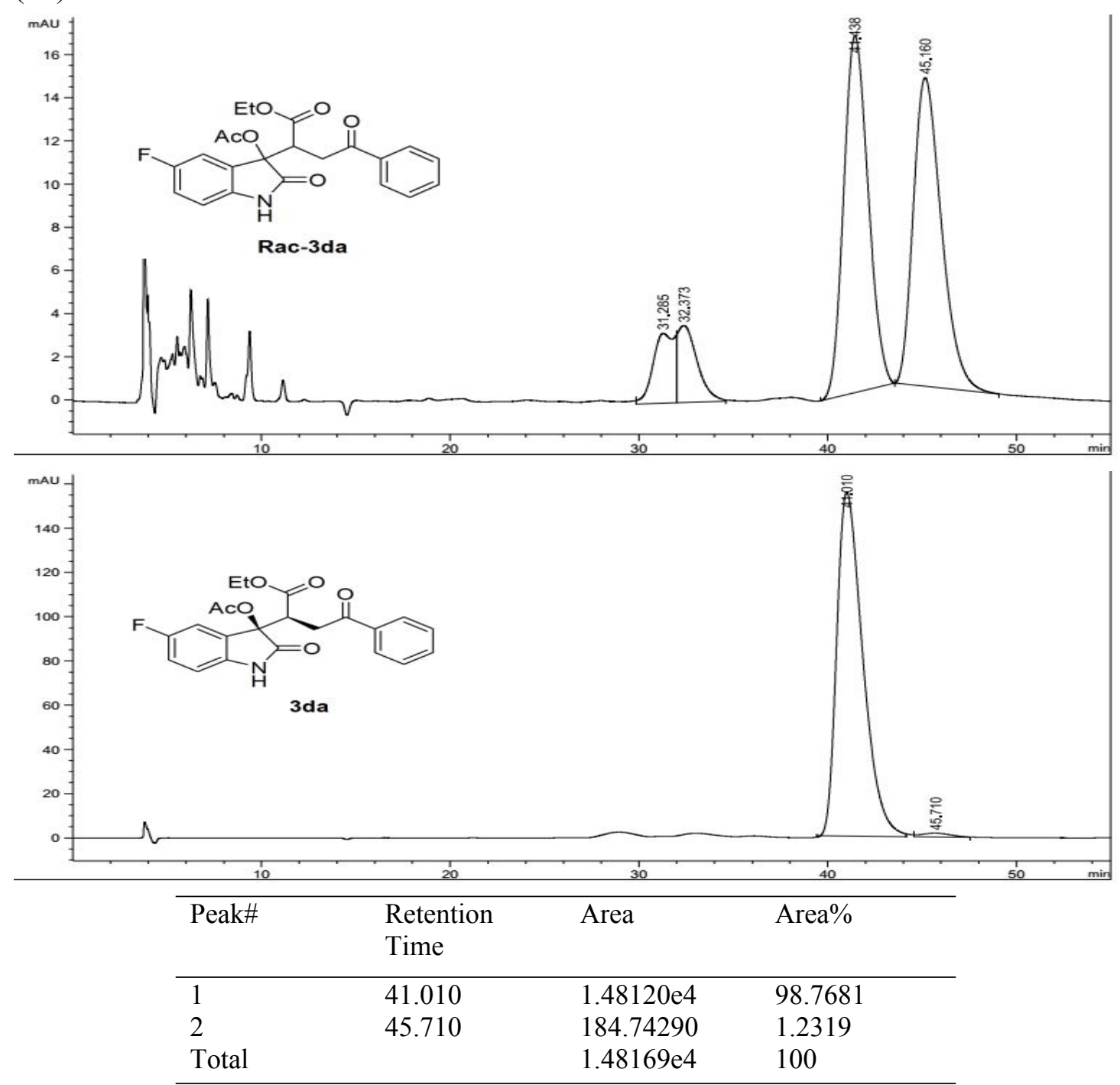


(22) 3ea

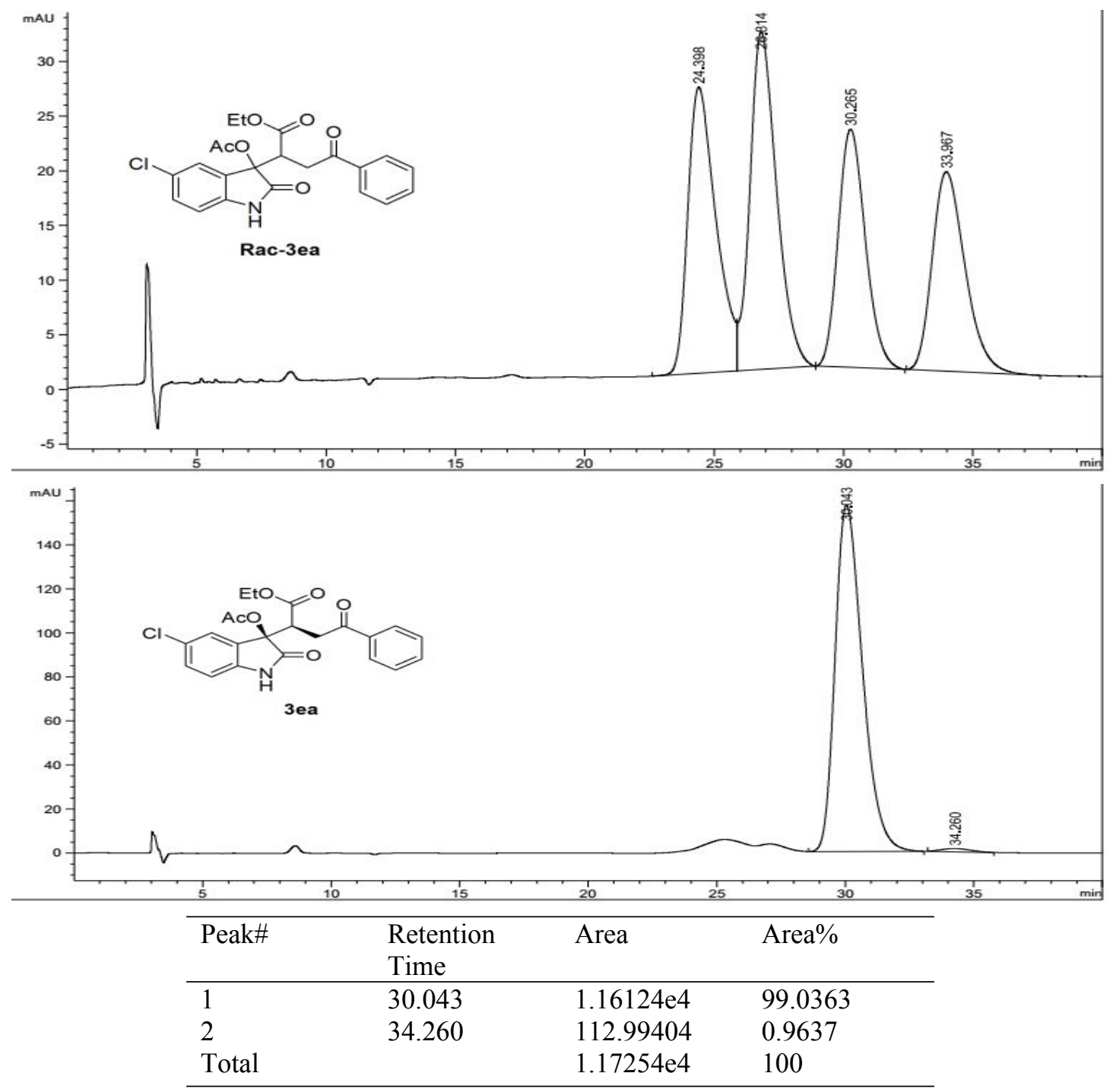


(23) $\mathbf{3 f a}$
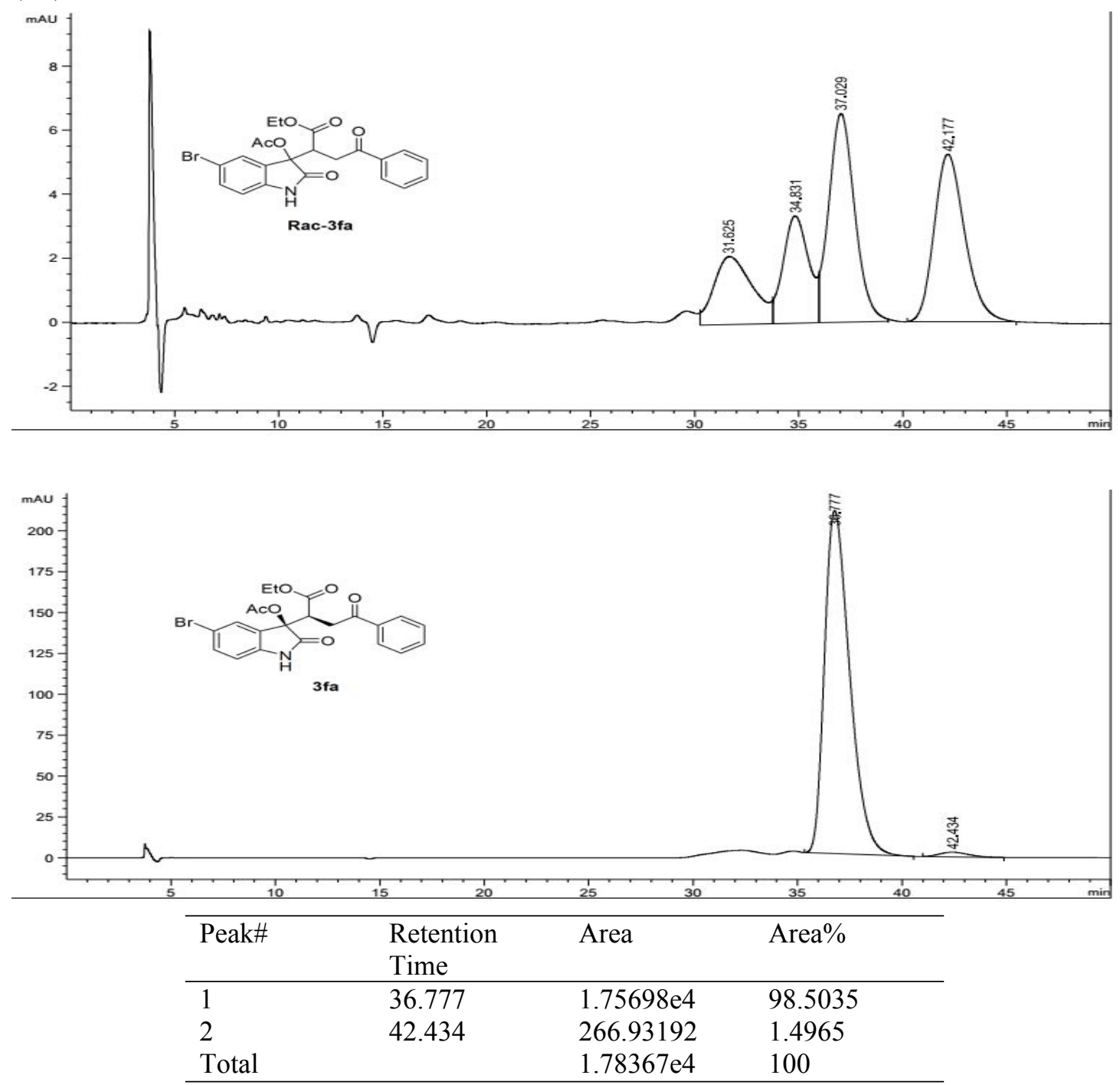

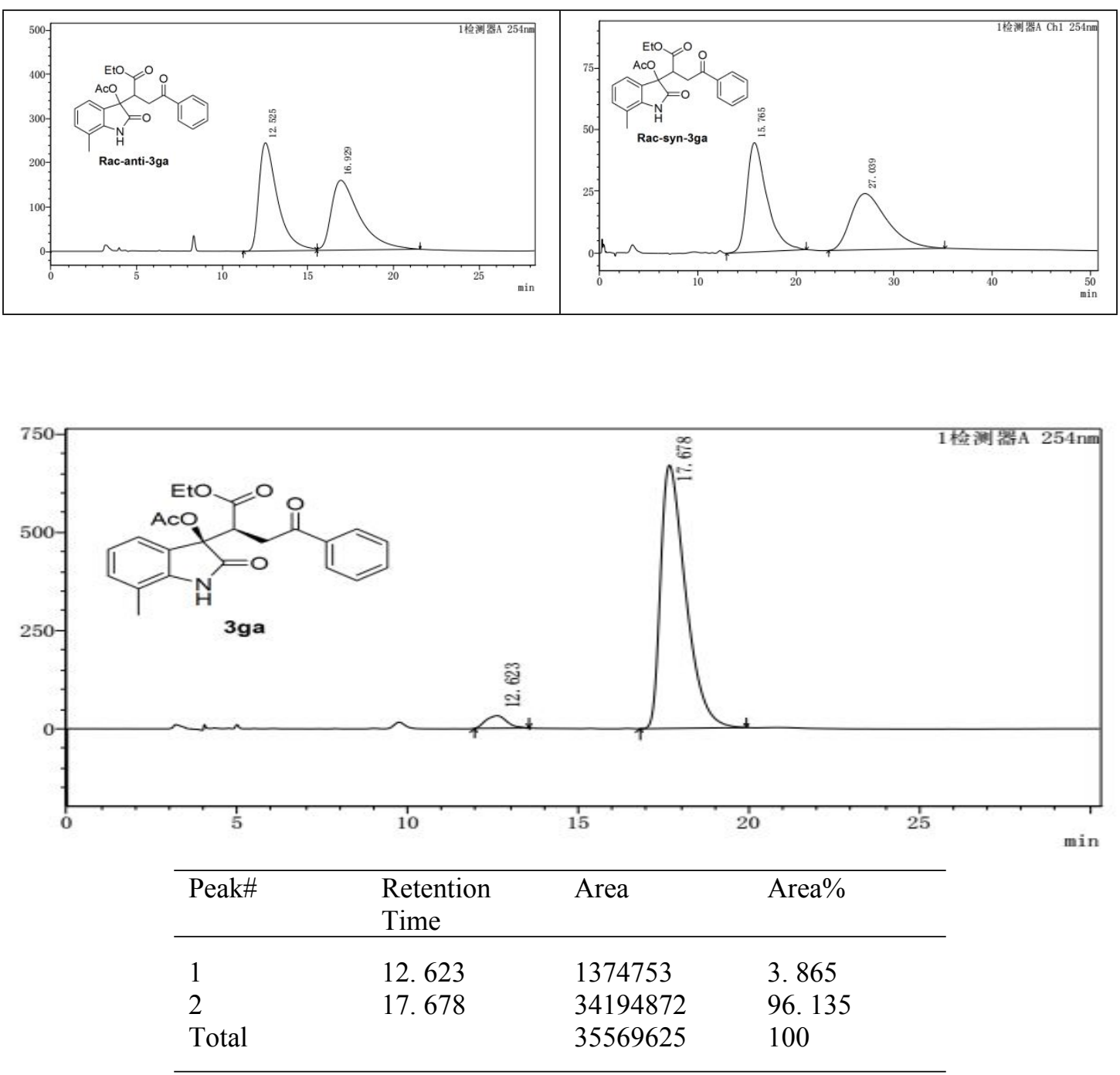
(25) 3fs
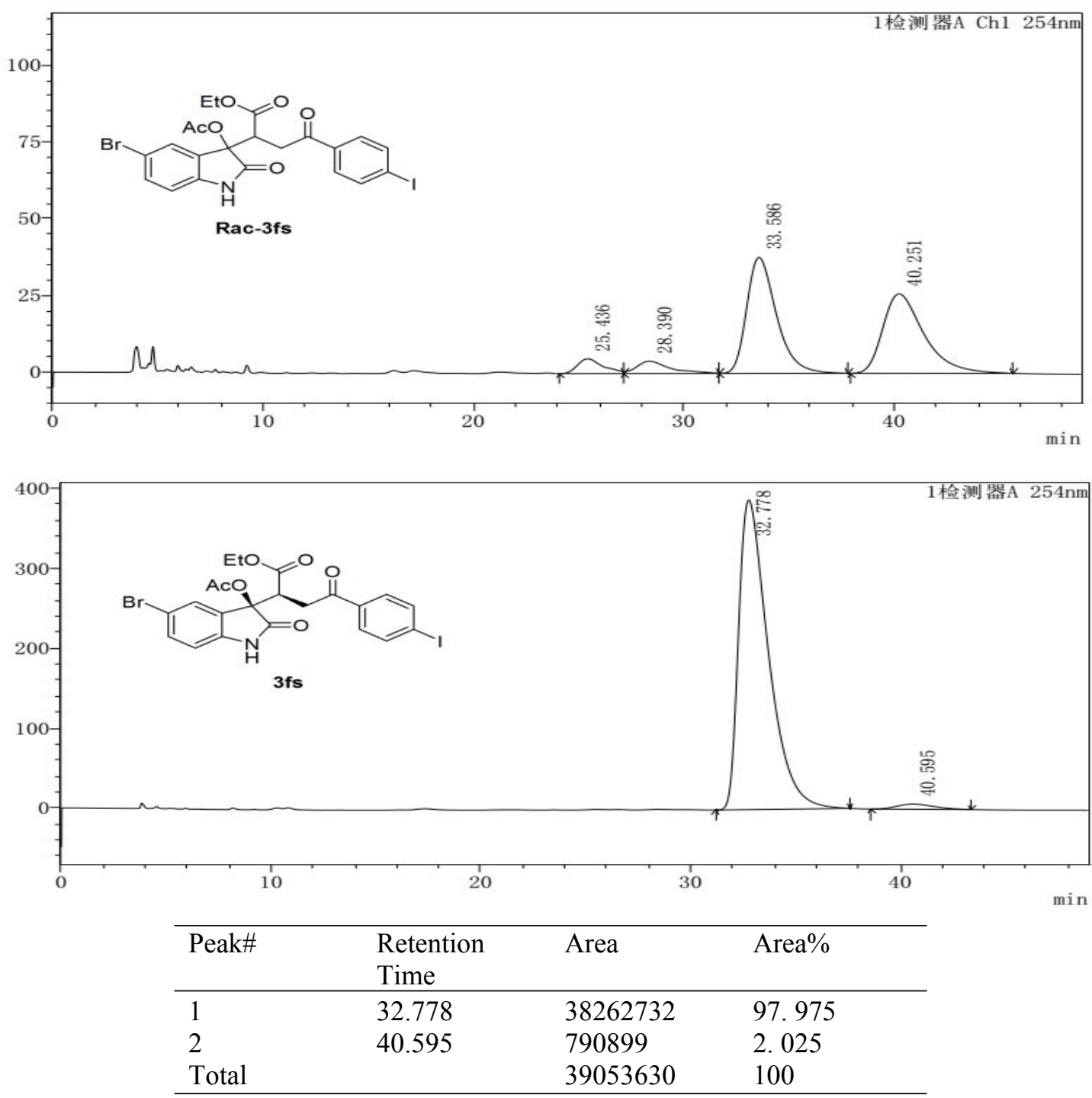
(26) 4aa
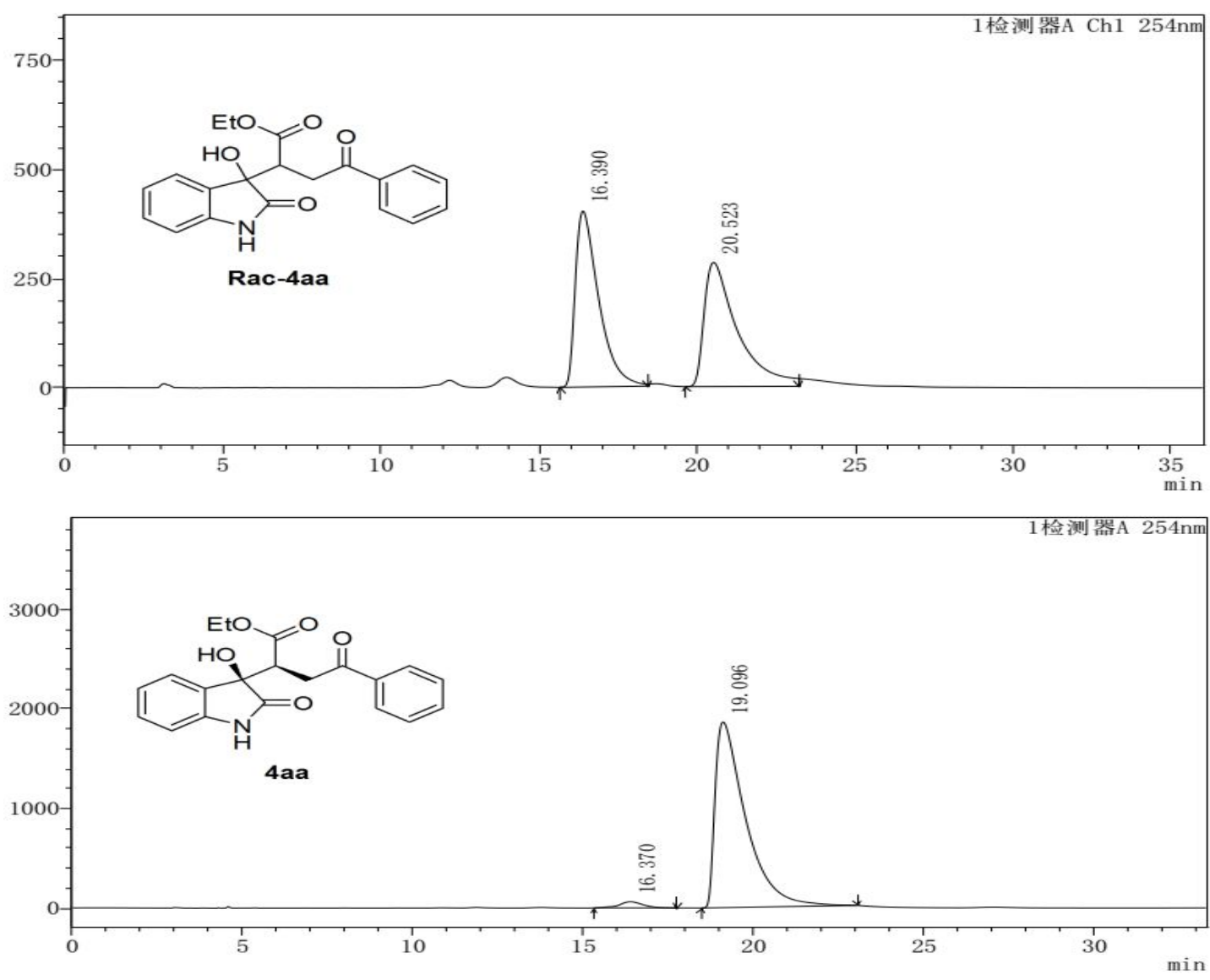

\begin{tabular}{llll}
\hline Peak\# & $\begin{array}{l}\text { Retention } \\
\text { Time }\end{array}$ & Area & Area\% \\
\hline 1 & 16.370 & 3074213 & 2.579 \\
2 & 19.096 & 116108469 & 97.421 \\
Total & & 119182682 & 100 \\
\hline
\end{tabular}


(27) 5aa
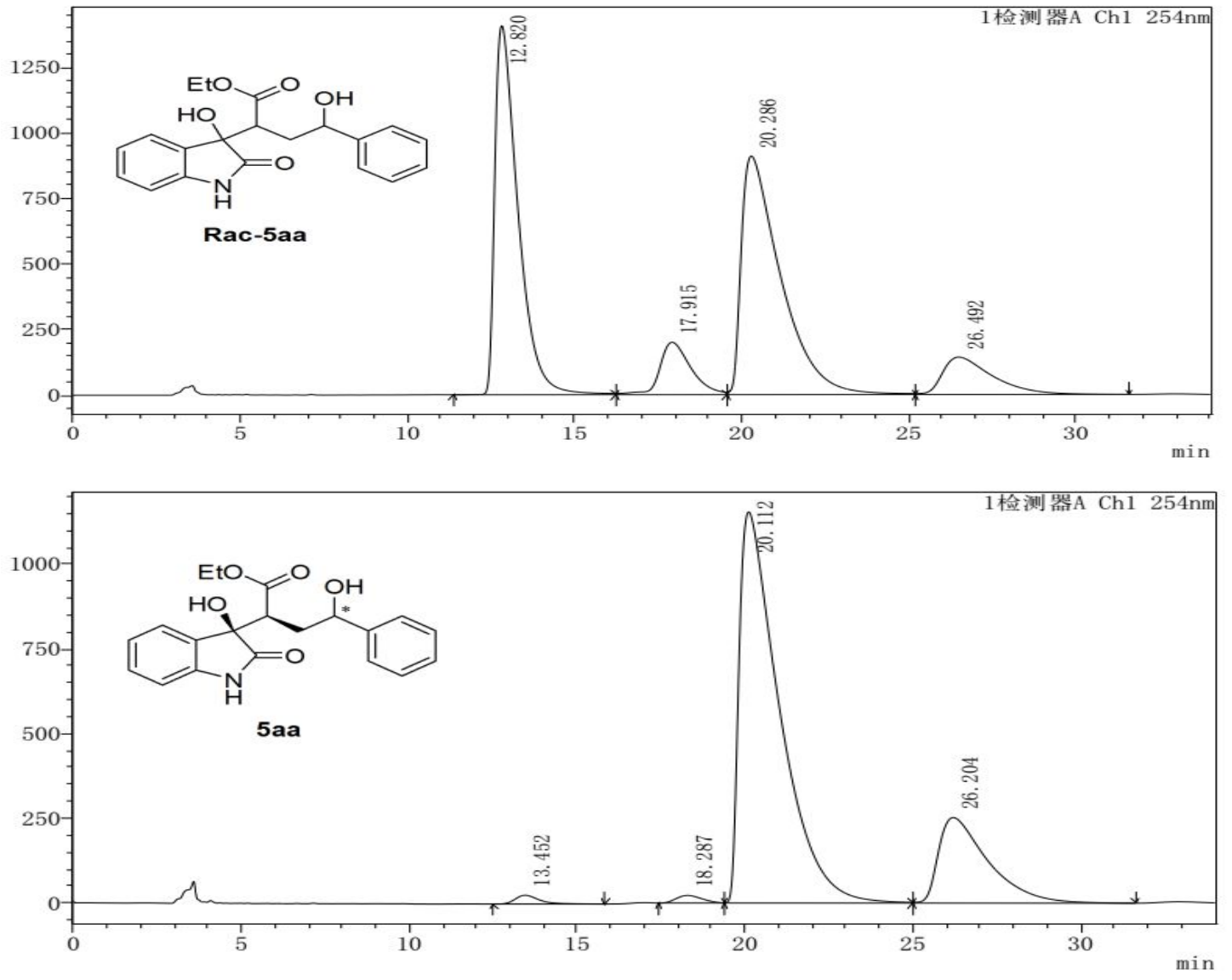

\begin{tabular}{llll}
\hline Peak\# & $\begin{array}{l}\text { Retention } \\
\text { Time }\end{array}$ & Area & Area\% \\
\hline 1 & 13.452 & 1289495 & 1.033 \\
2 & 18.287 & 1190487 & 0.954 \\
3 & 20.112 & 96365840 & 77.216 \\
4 & 26.204 & 25954882 & 20.797 \\
Total & & 124800704 & 100 \\
\hline
\end{tabular}


(28) 4fs
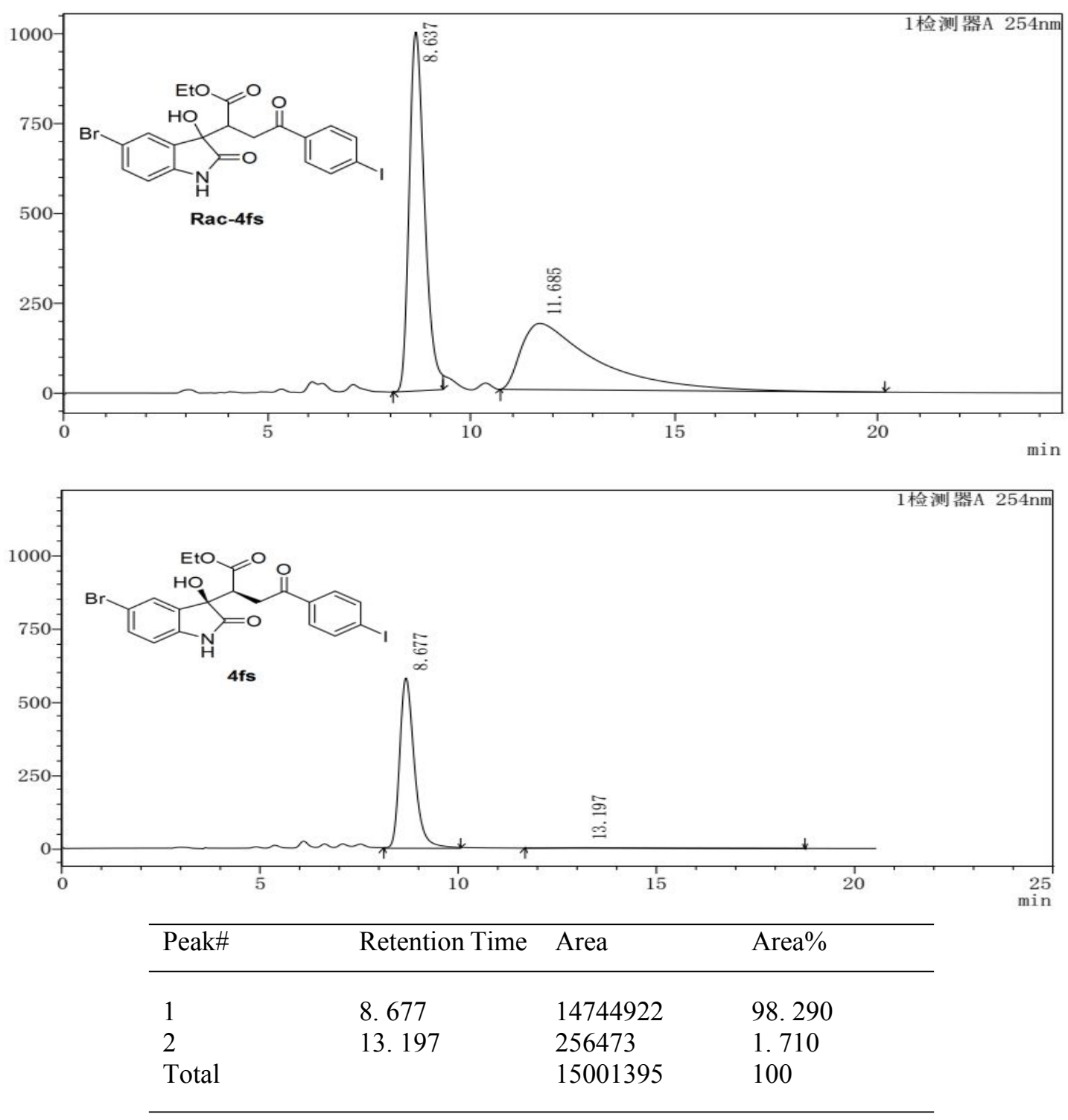
7. Copies of NMR Spectra

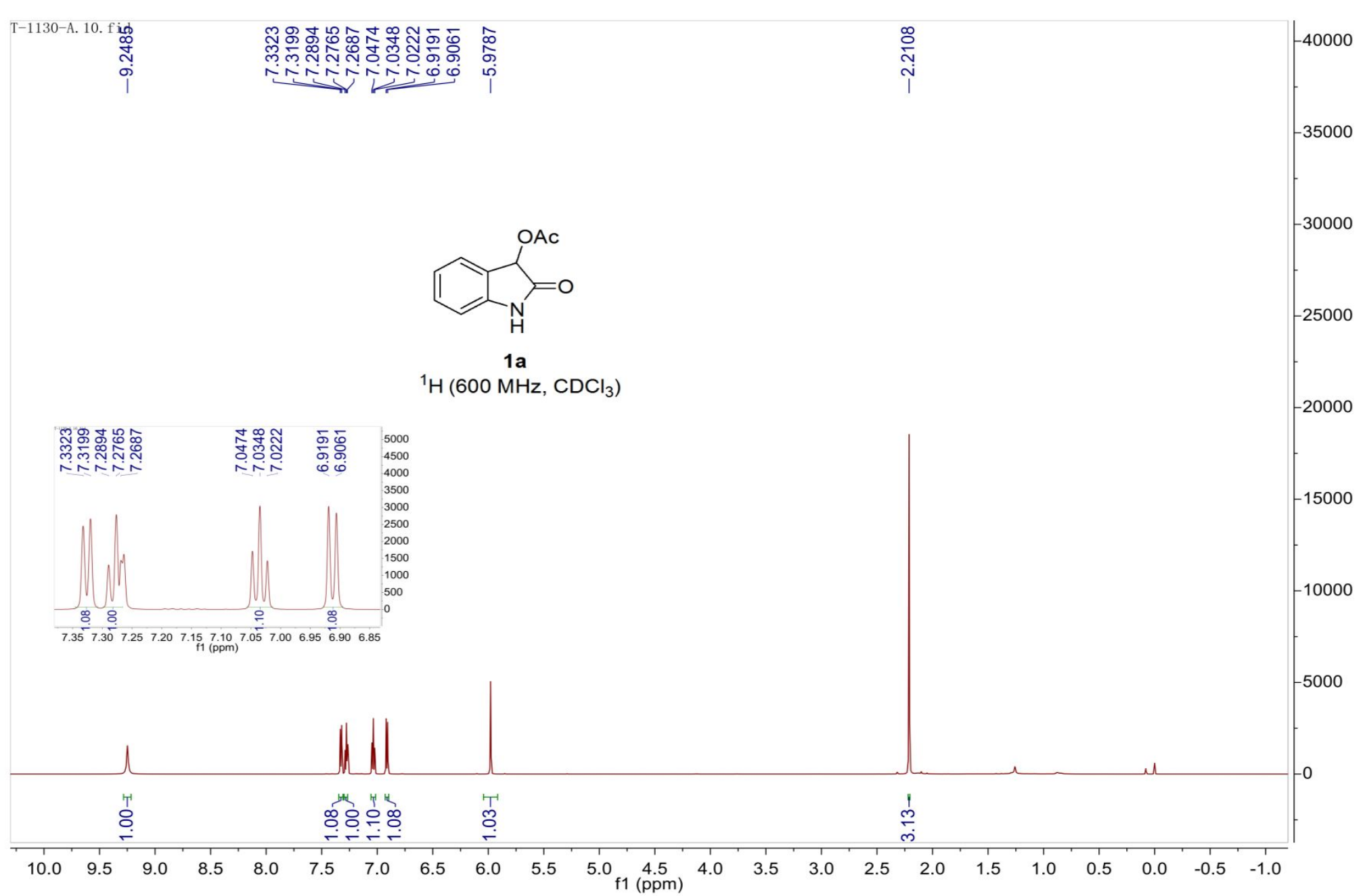




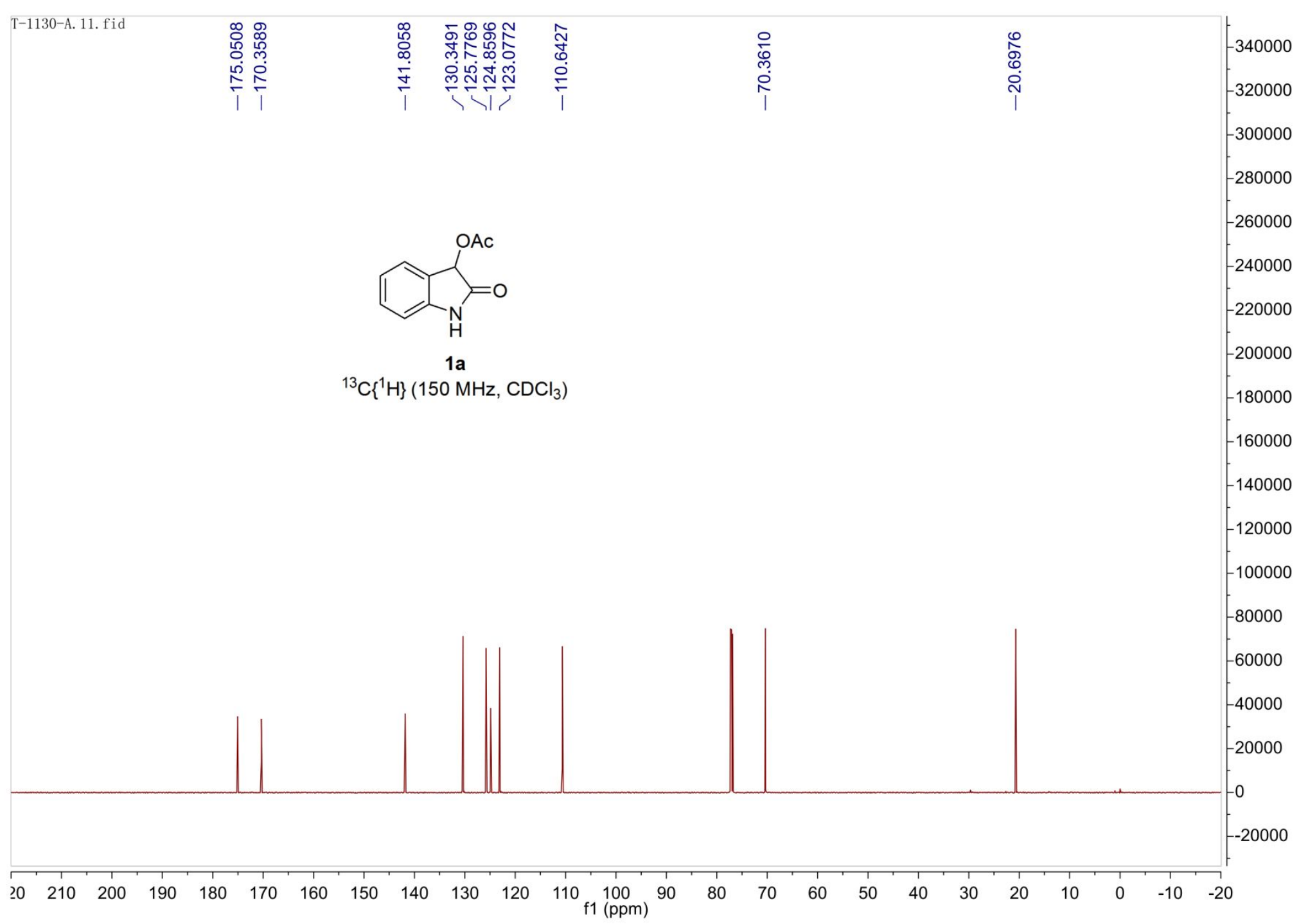




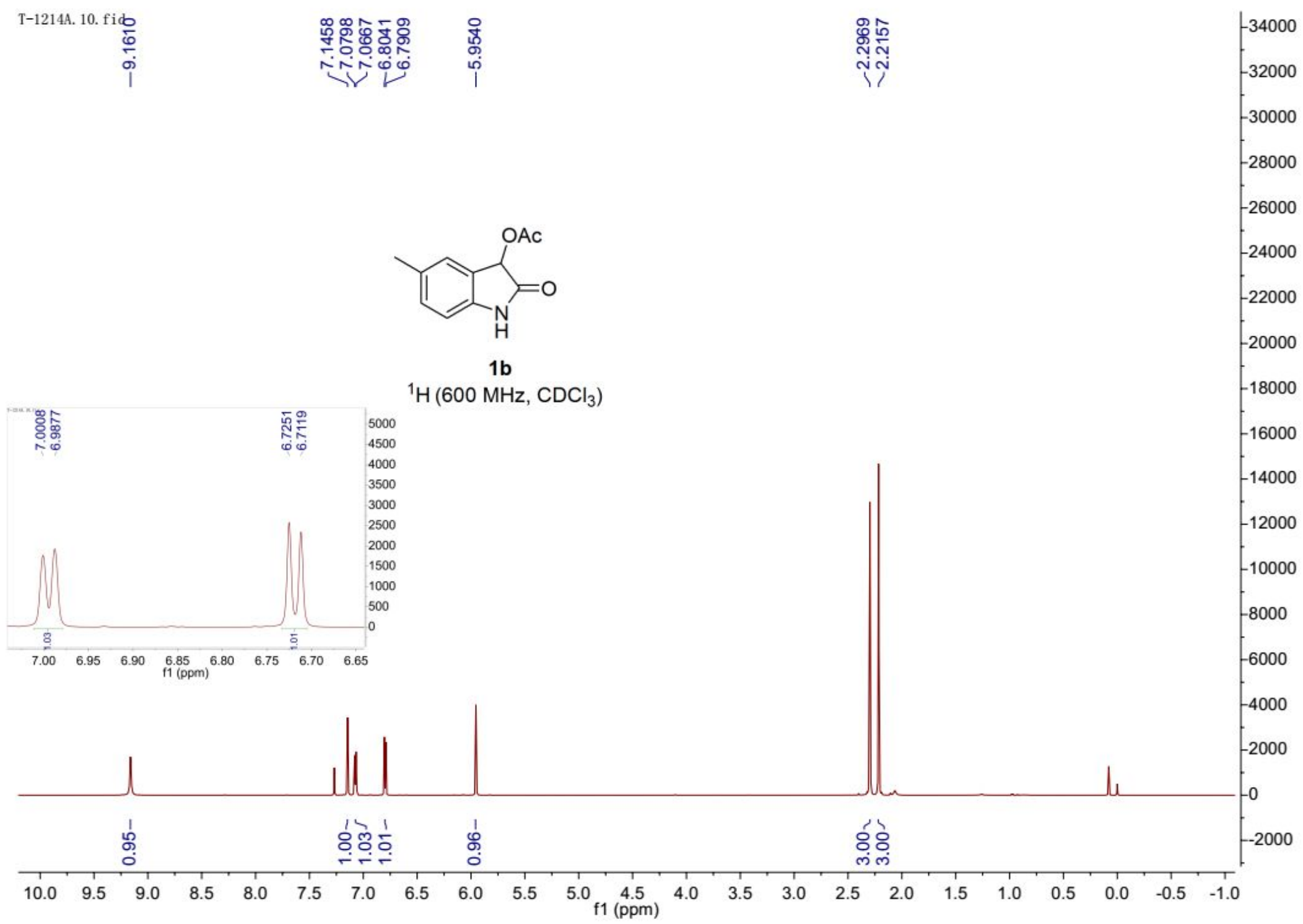




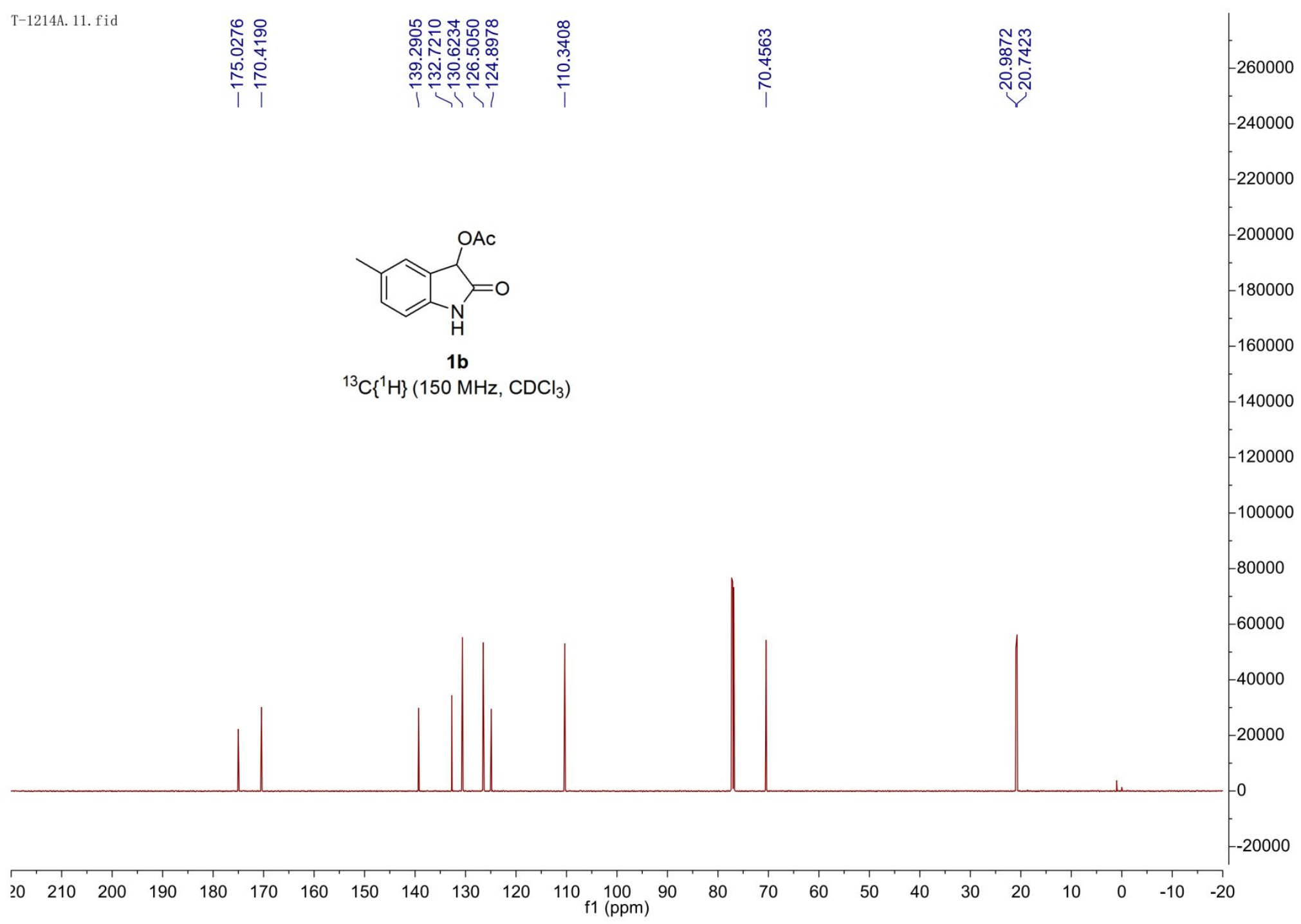




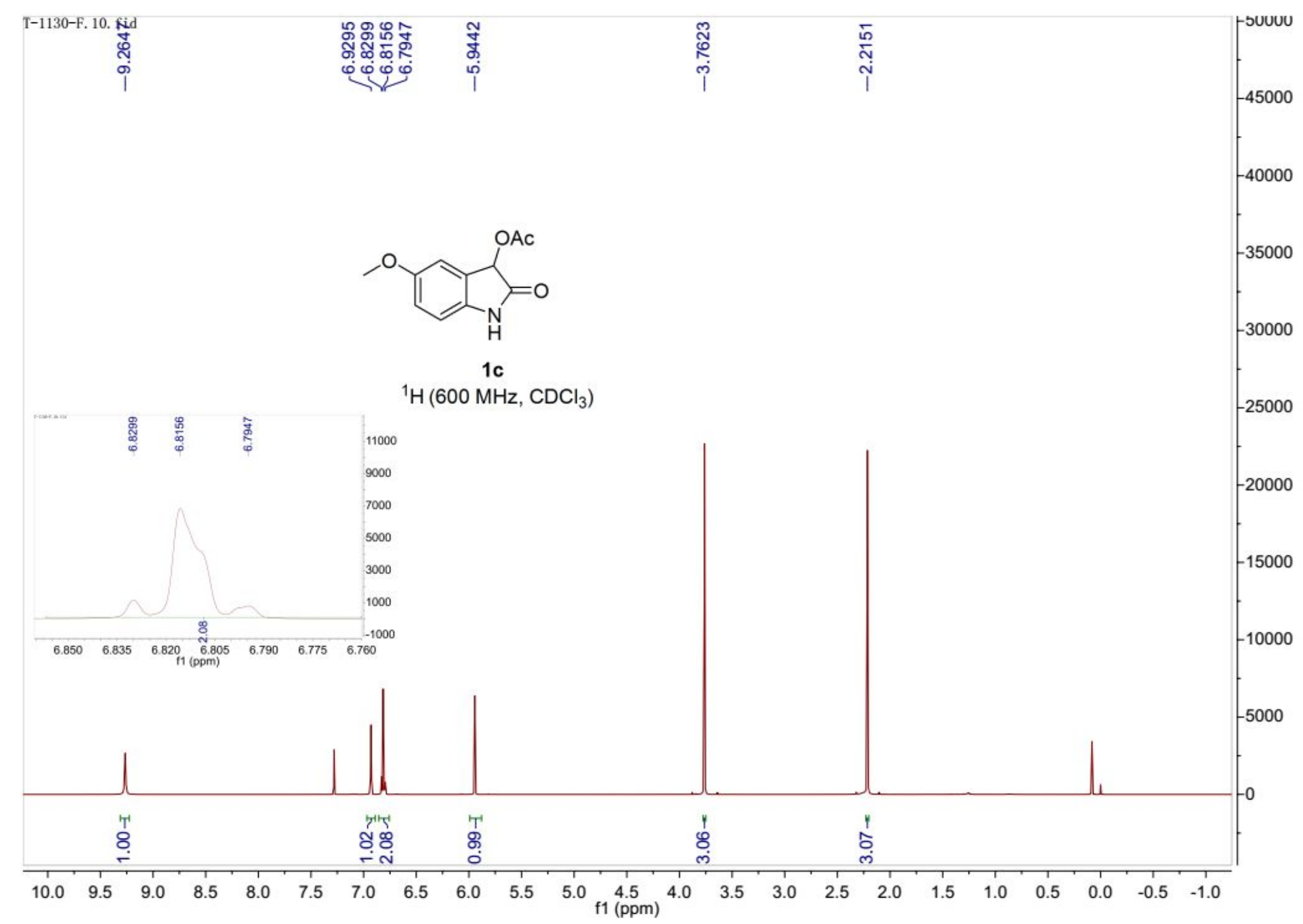




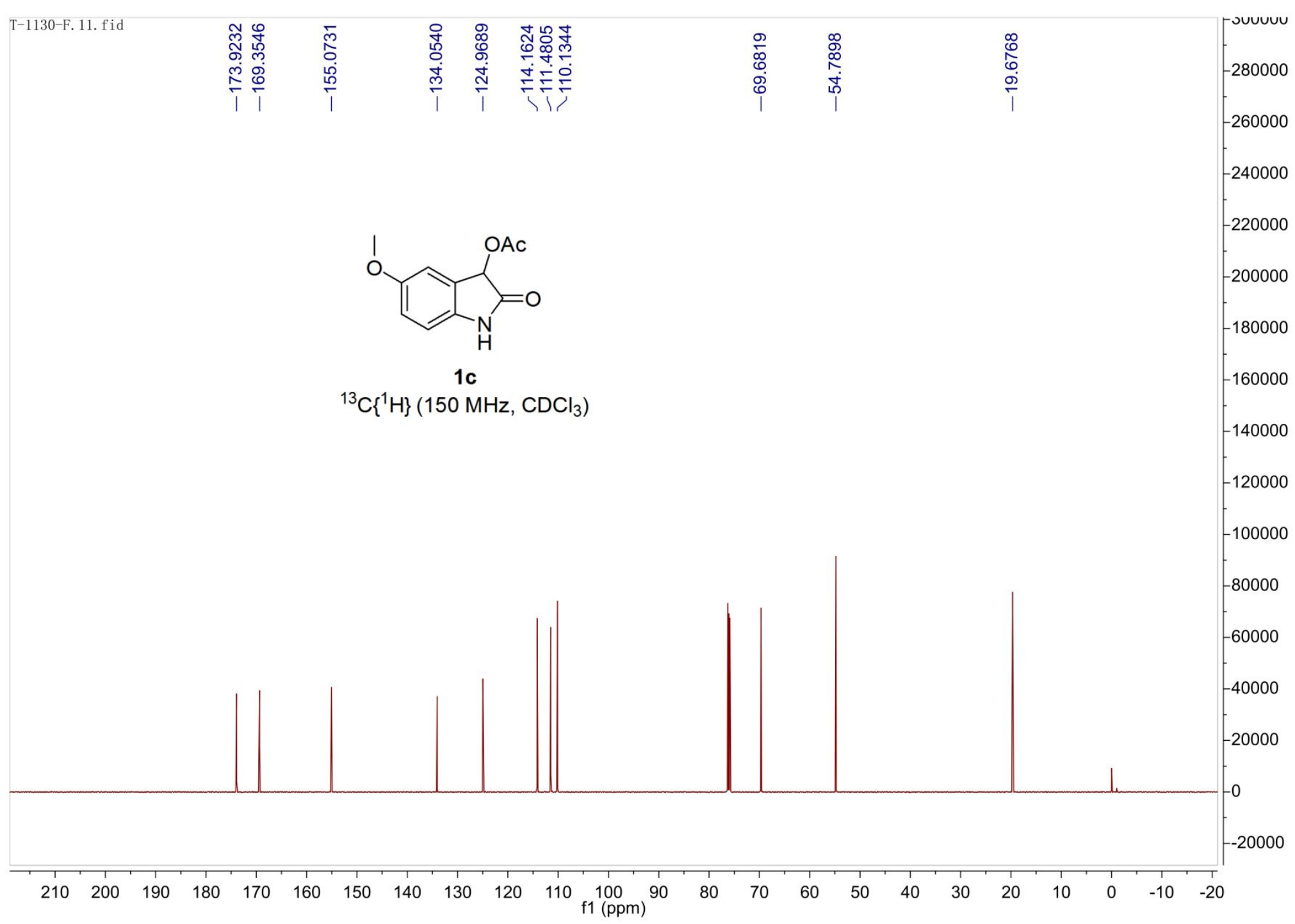




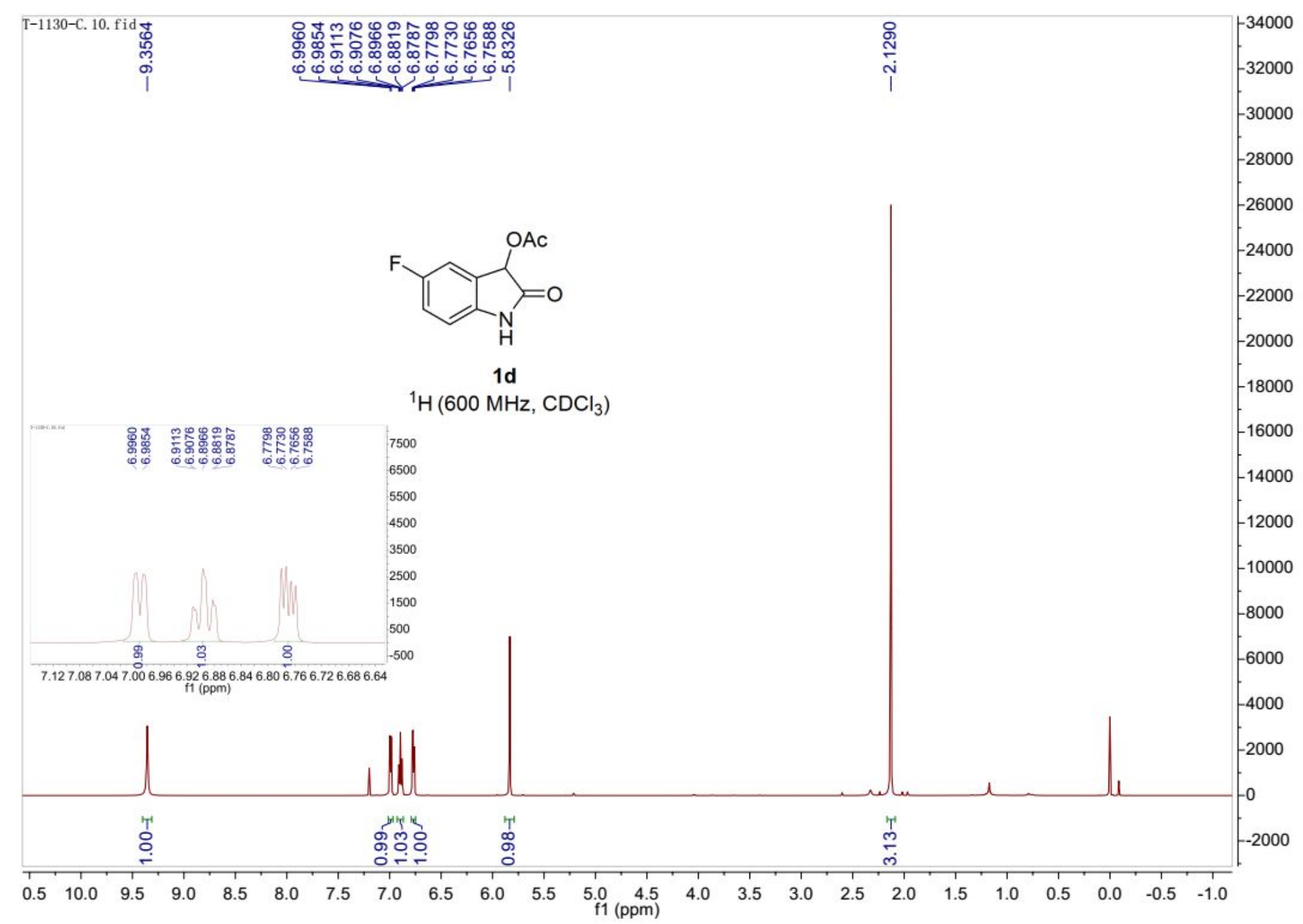




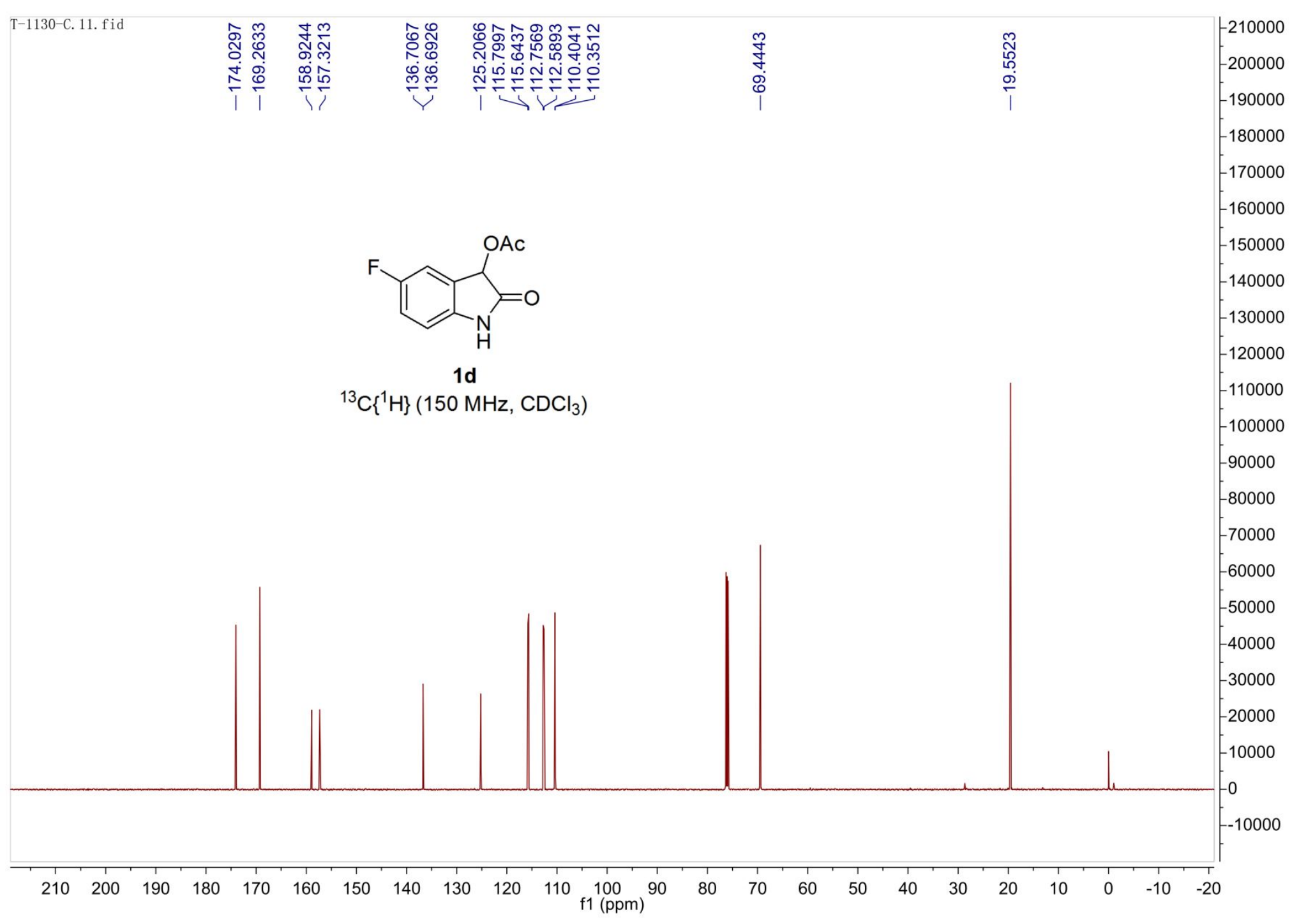


T-4-12. 13. fid

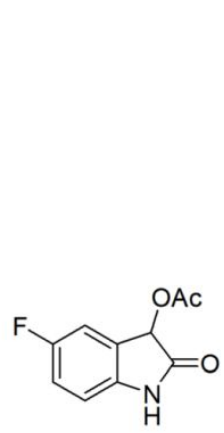

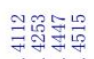

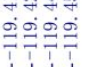

1d

${ }^{19} \mathrm{~F}\left(565 \mathrm{MHz}, \mathrm{CDCl}_{3}\right)$

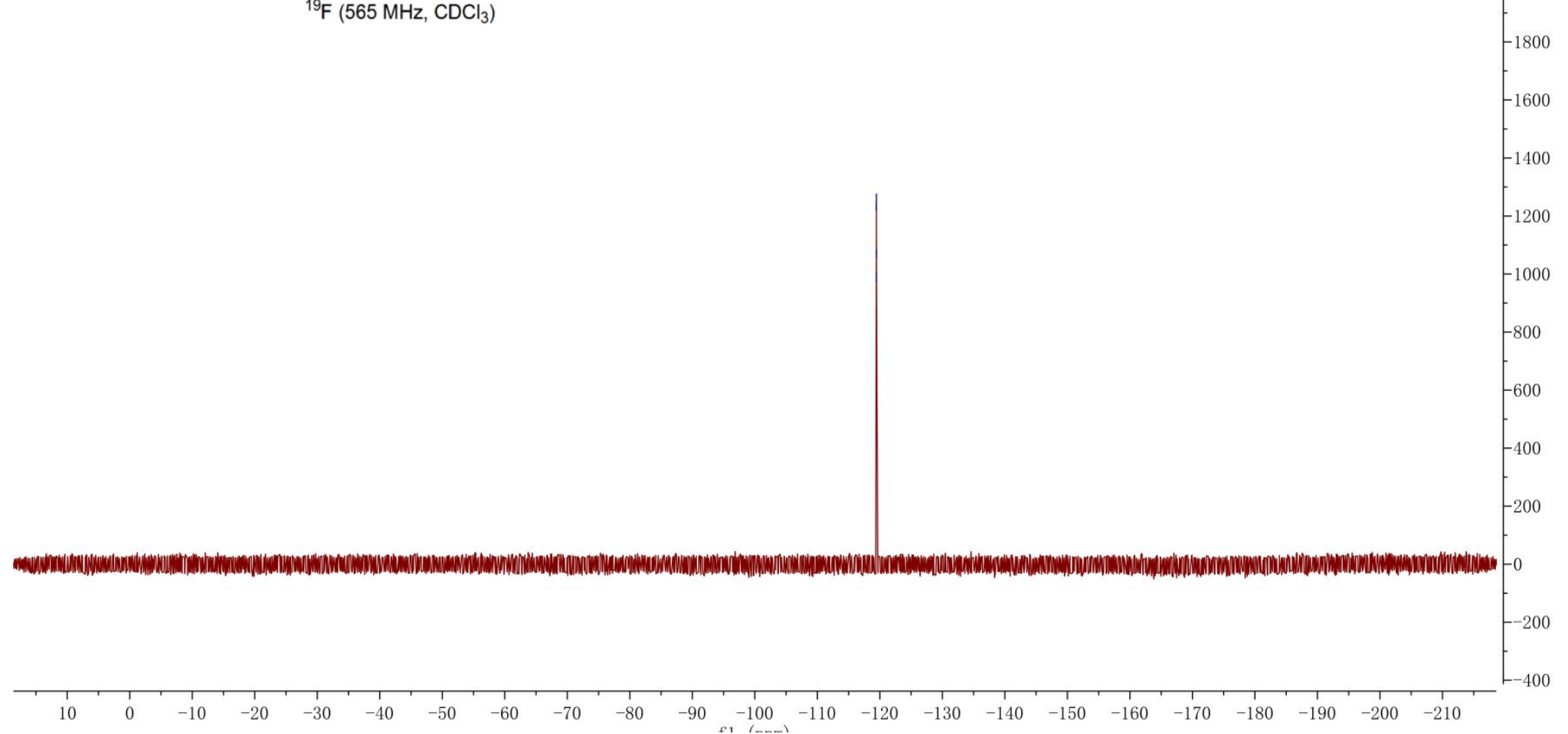




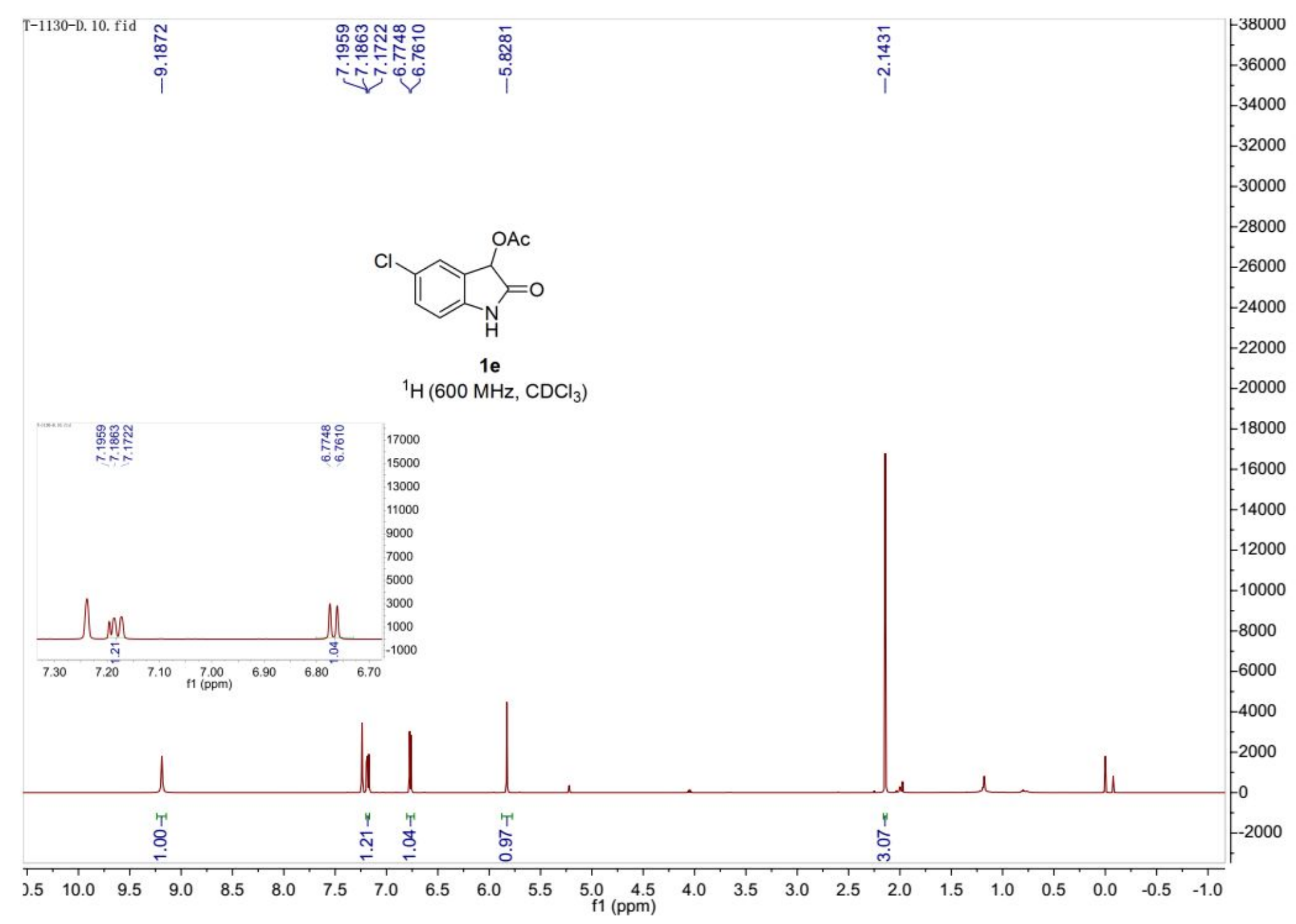




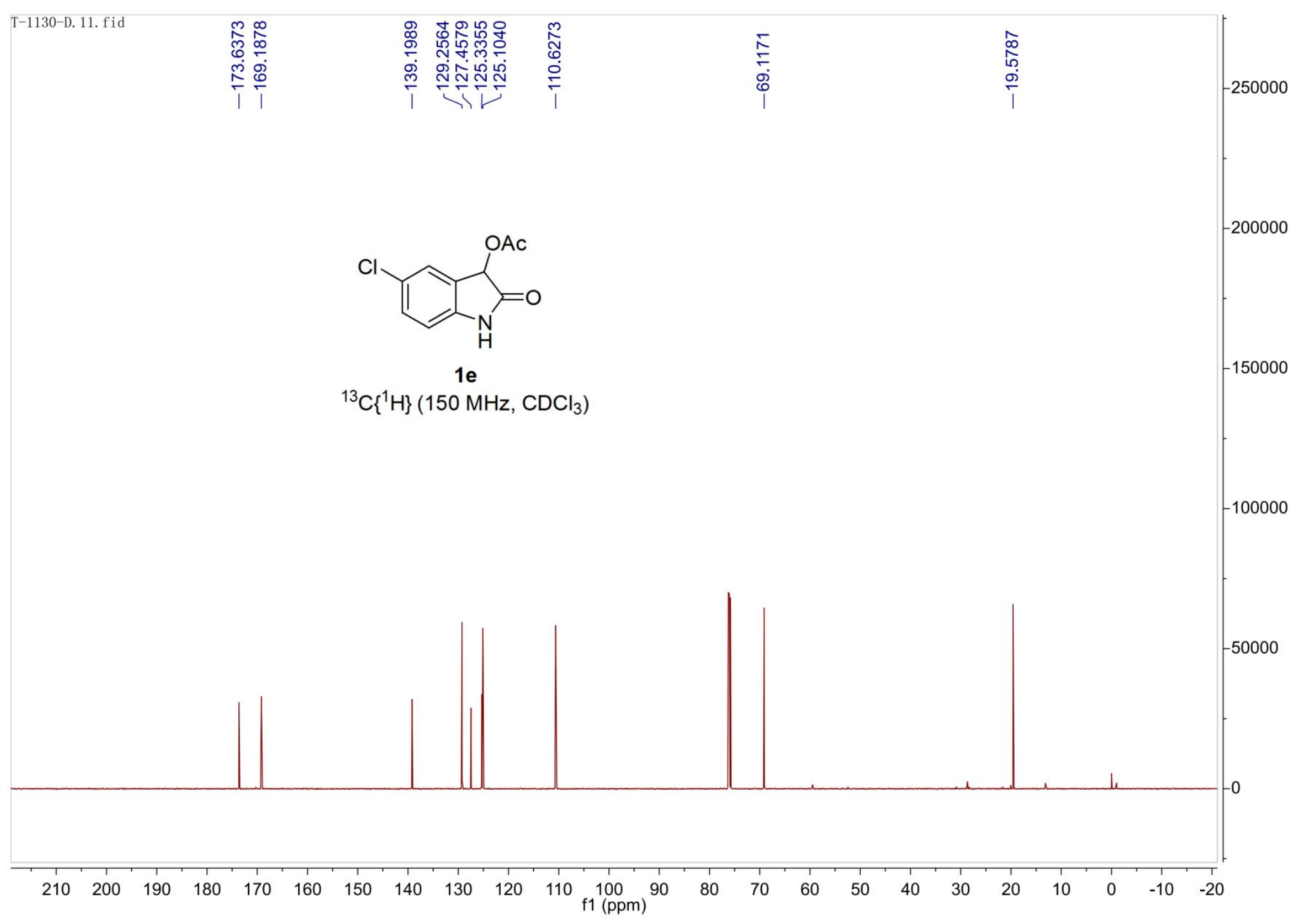




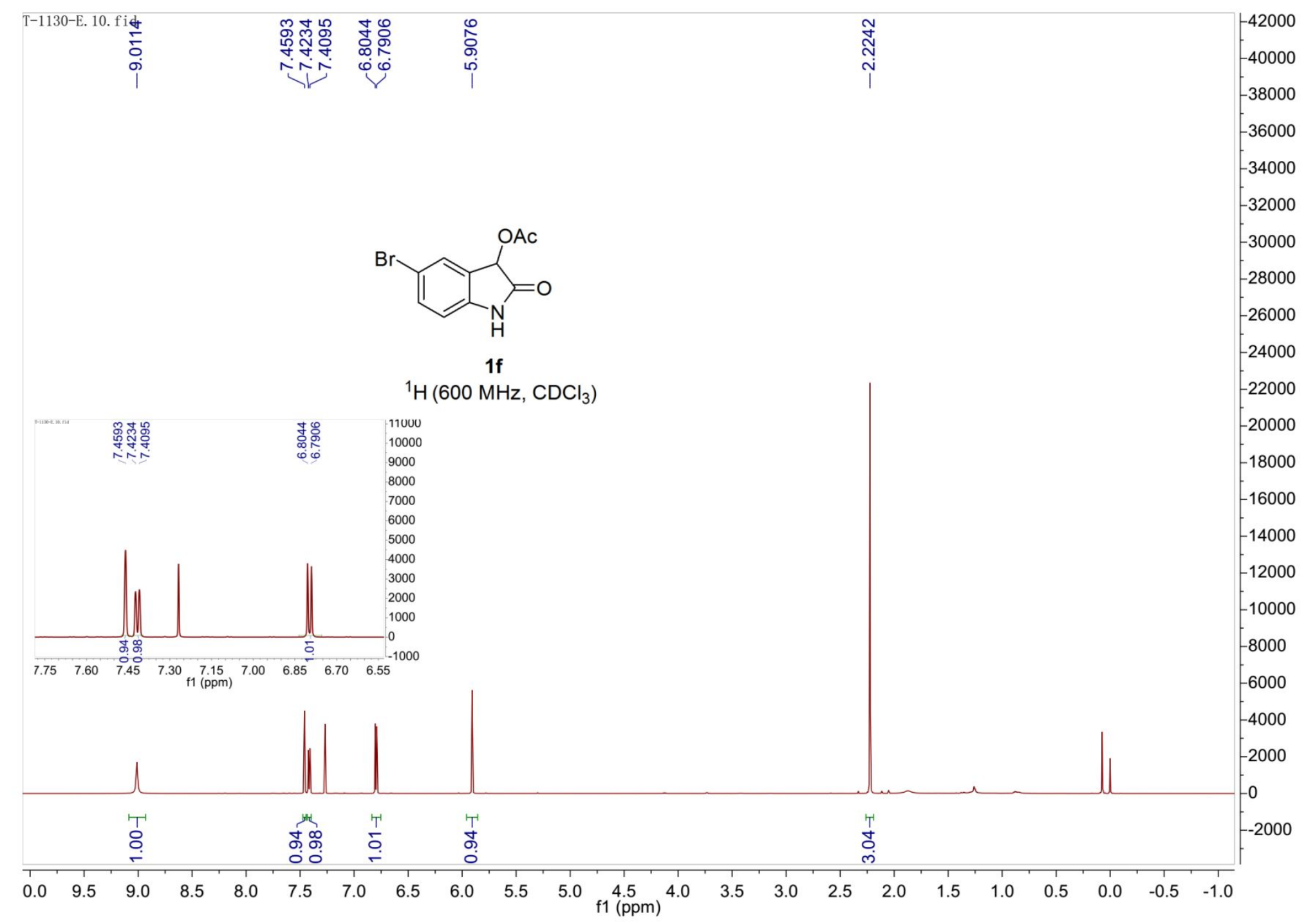




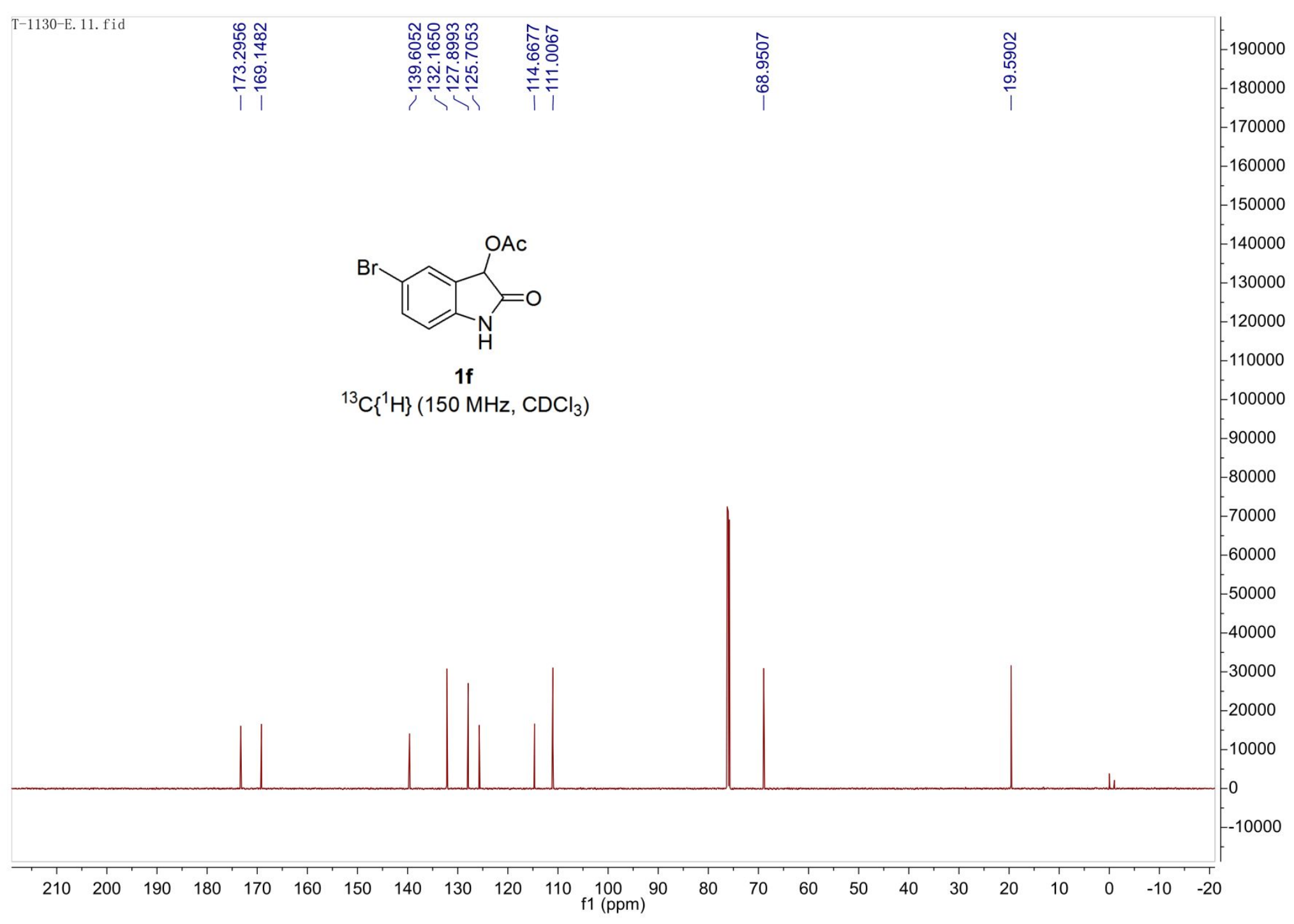




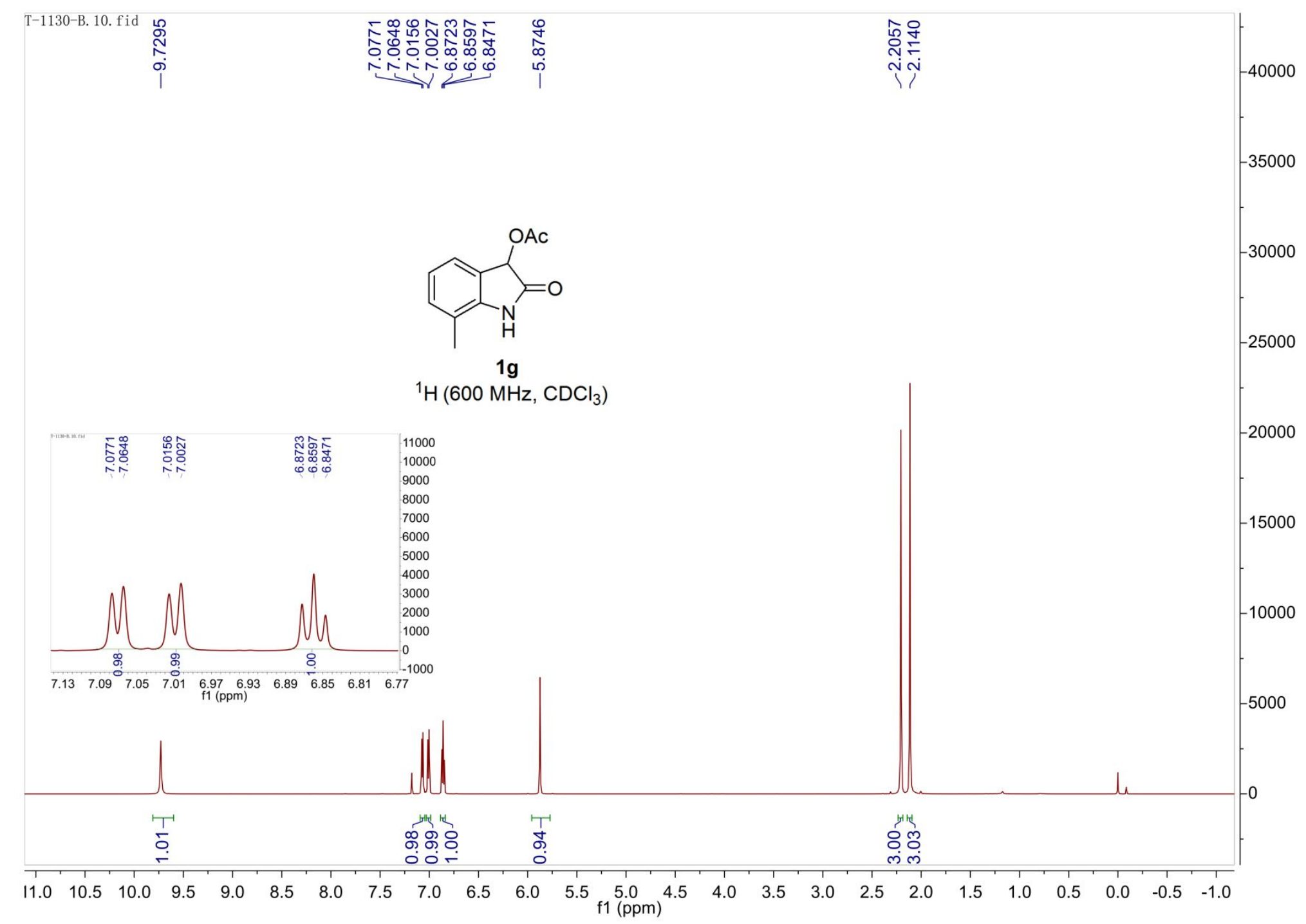




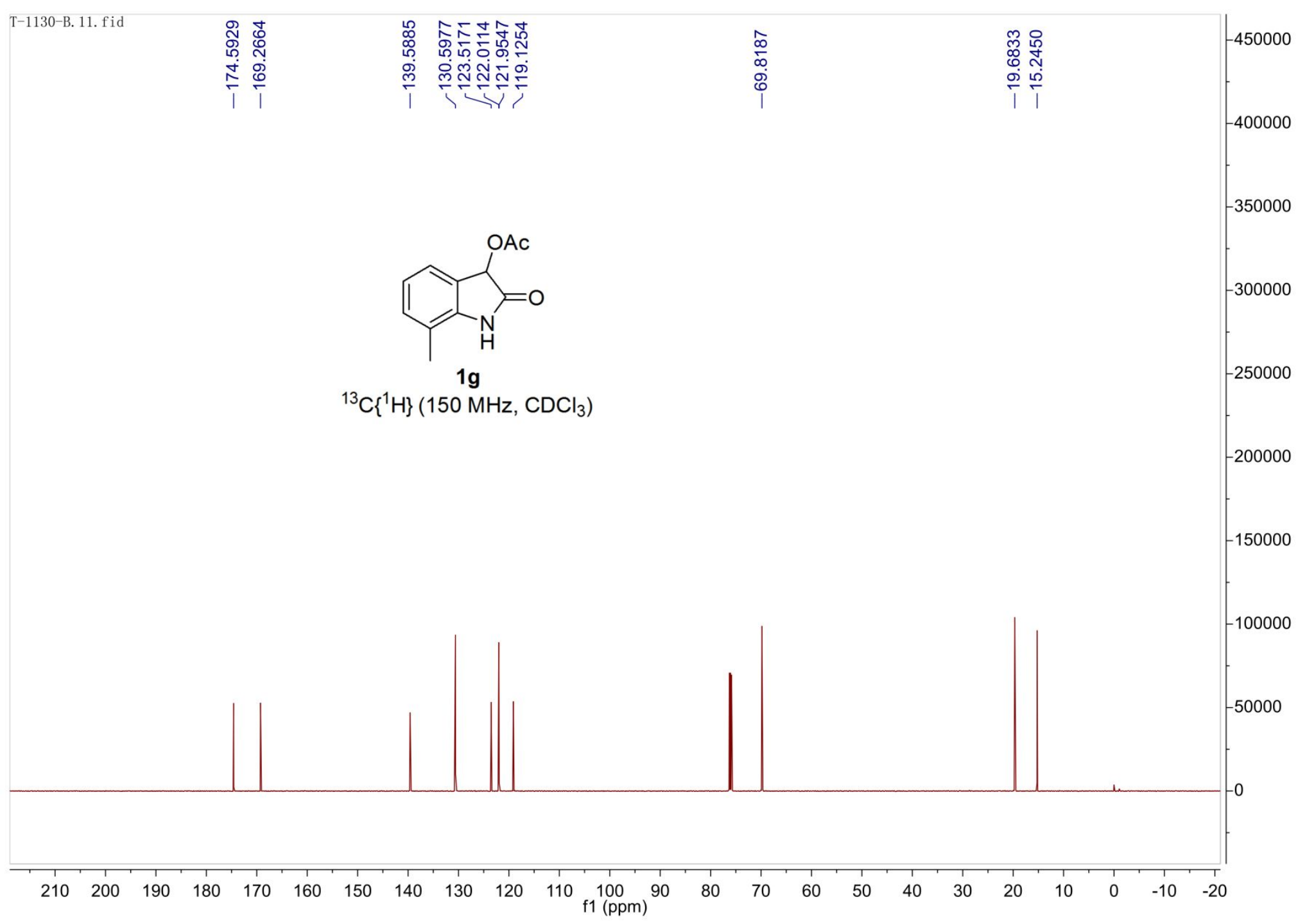




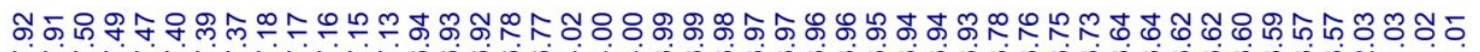

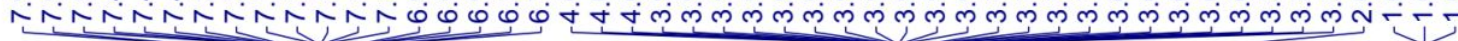

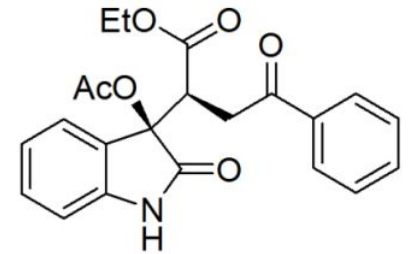

3aa

${ }^{1} \mathrm{H}\left(600 \mathrm{MHz}, \mathrm{CDCl}_{3}\right)$

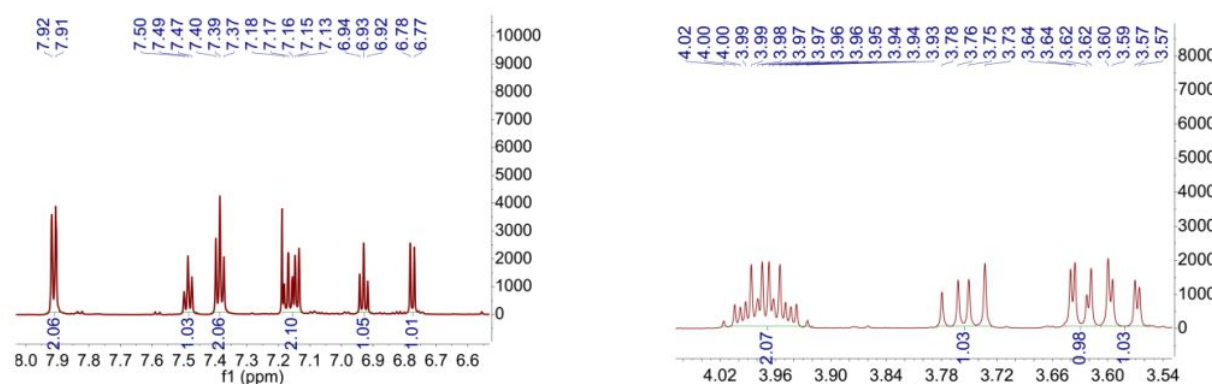

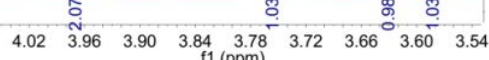

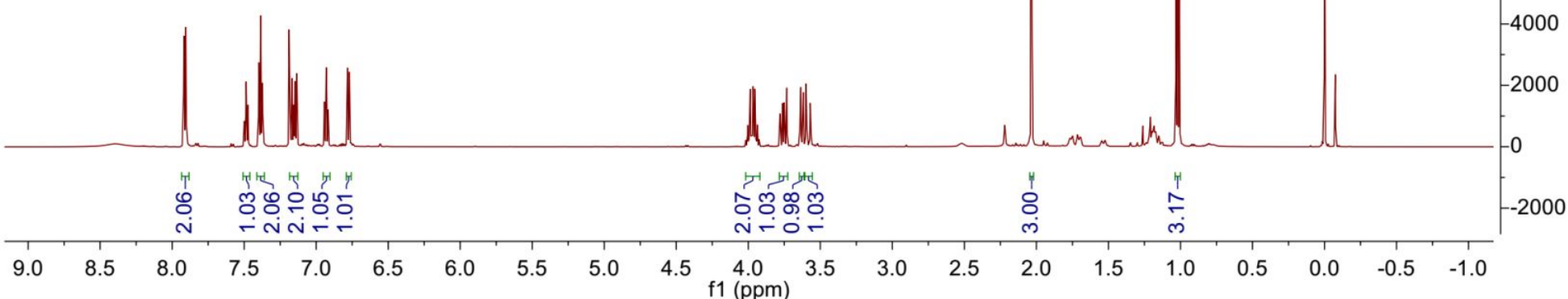




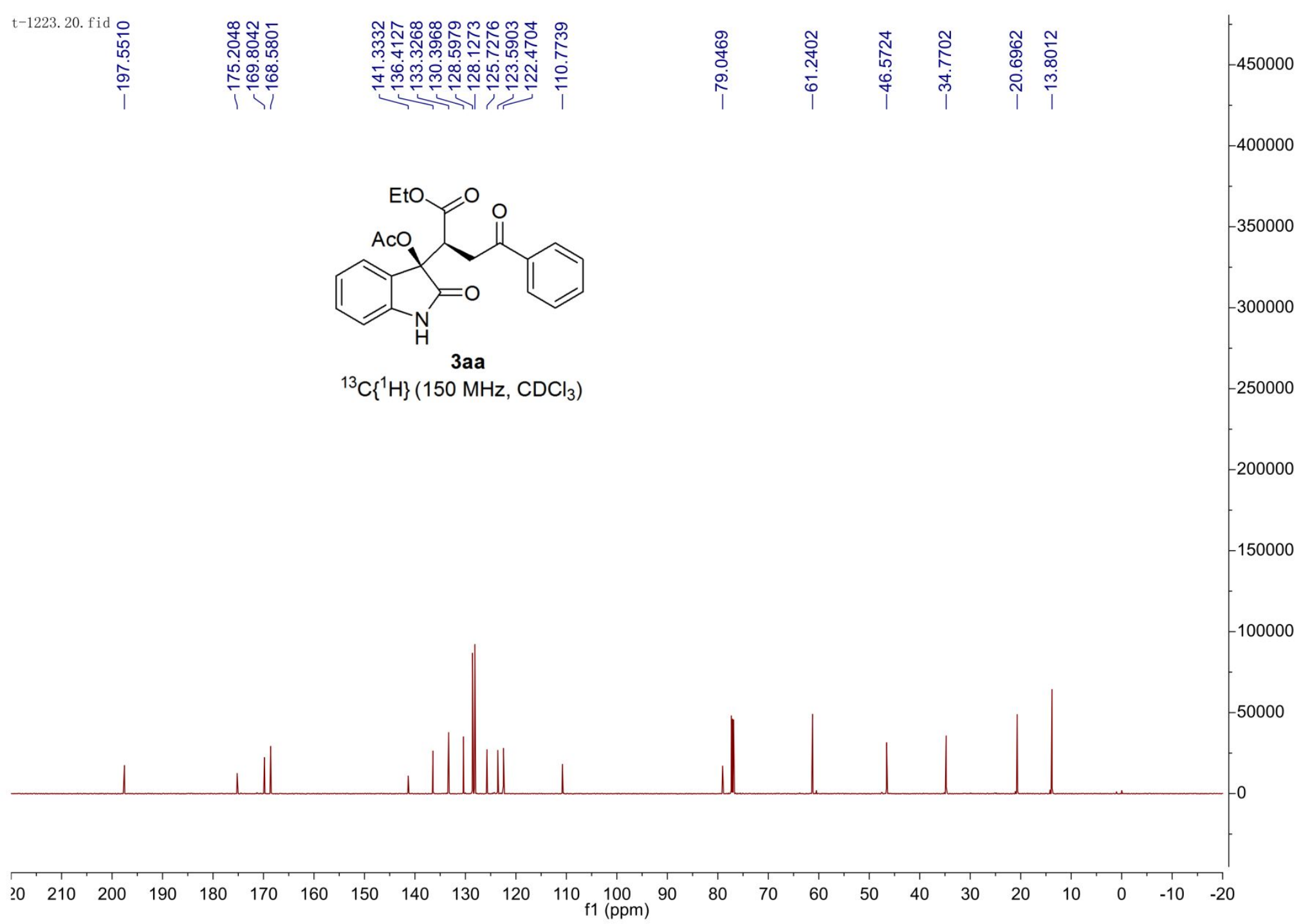




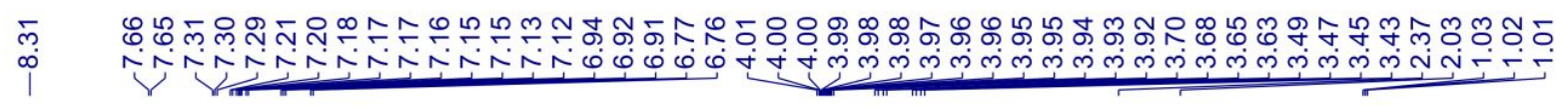

40000
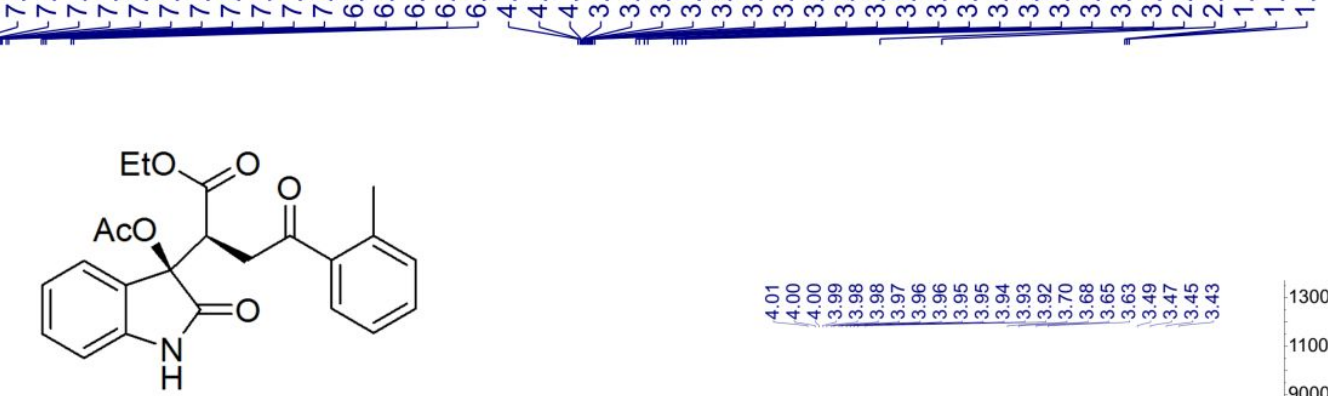

$3 a b$

${ }^{1} \mathrm{H}\left(600 \mathrm{MHz}, \mathrm{CDCl}_{3}\right)$

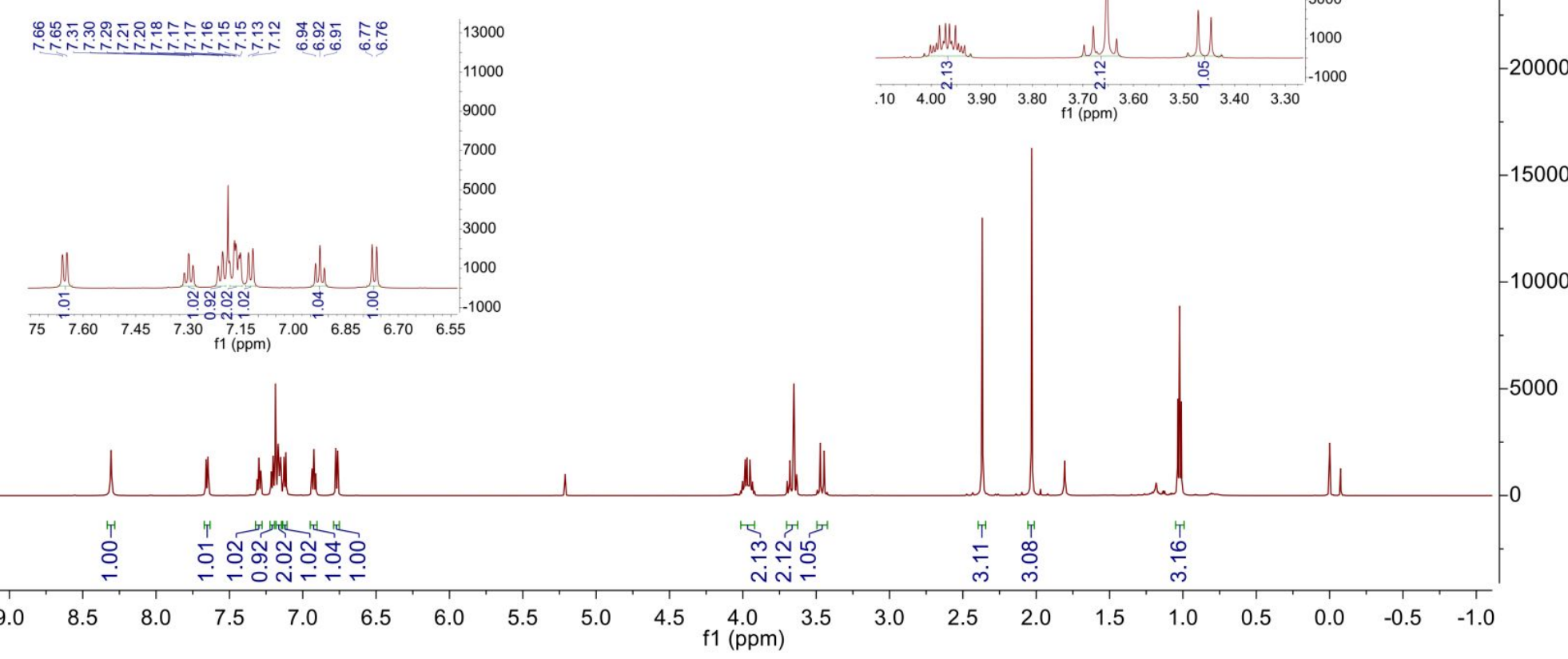




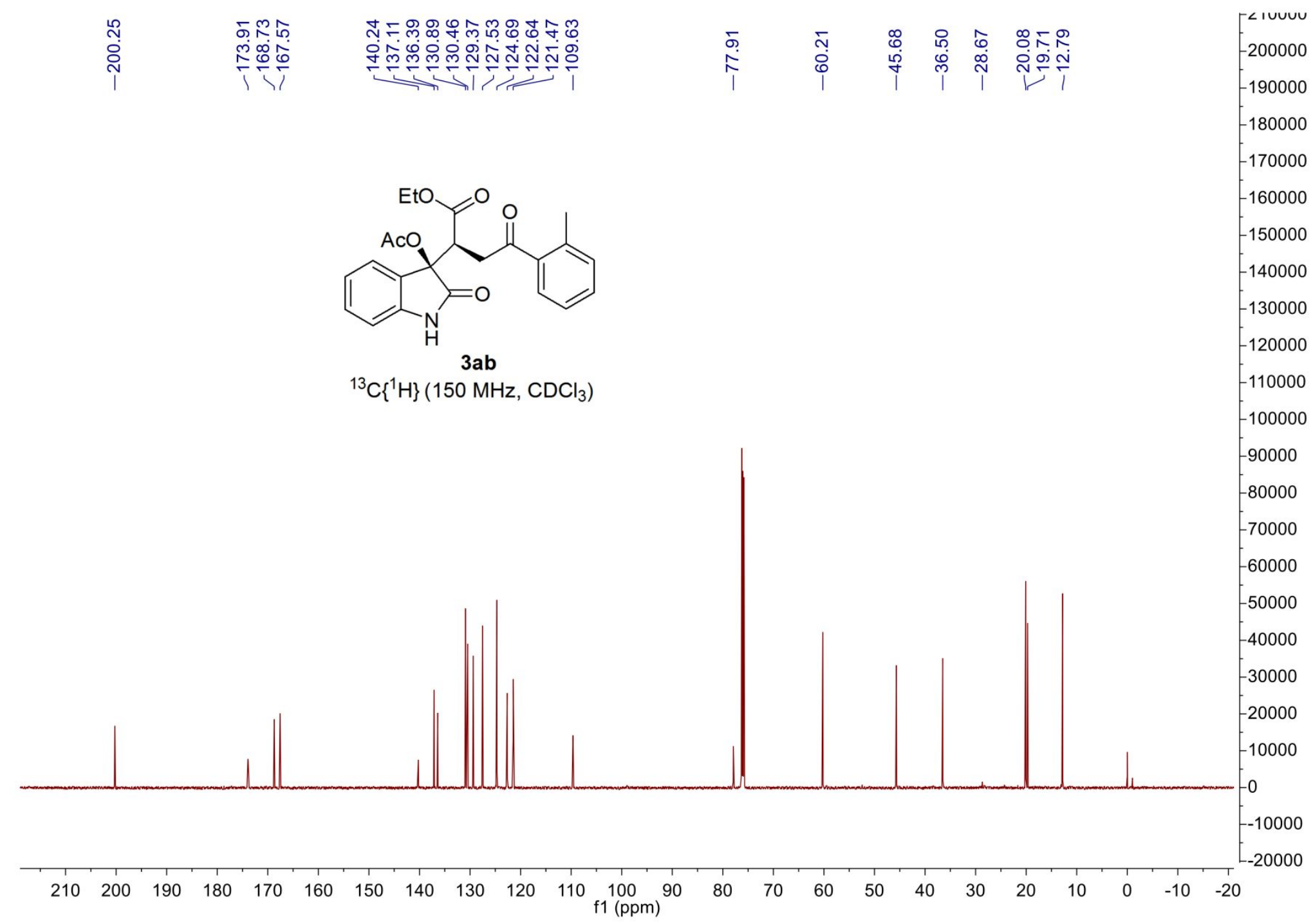




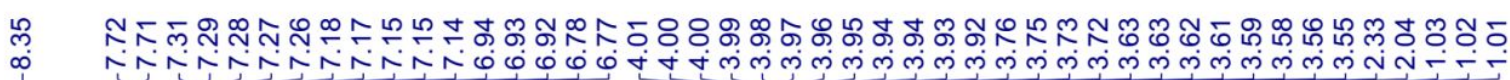

90000<smiles>CCOC(=O)[C@H](CC(=O)c1cccc(C)c1)[C@]1(OC(C)=O)C(=O)Nc2ccccc21</smiles>

3ac

${ }^{1} \mathrm{H}\left(600 \mathrm{MHz}, \mathrm{CDCl}_{3}\right)$
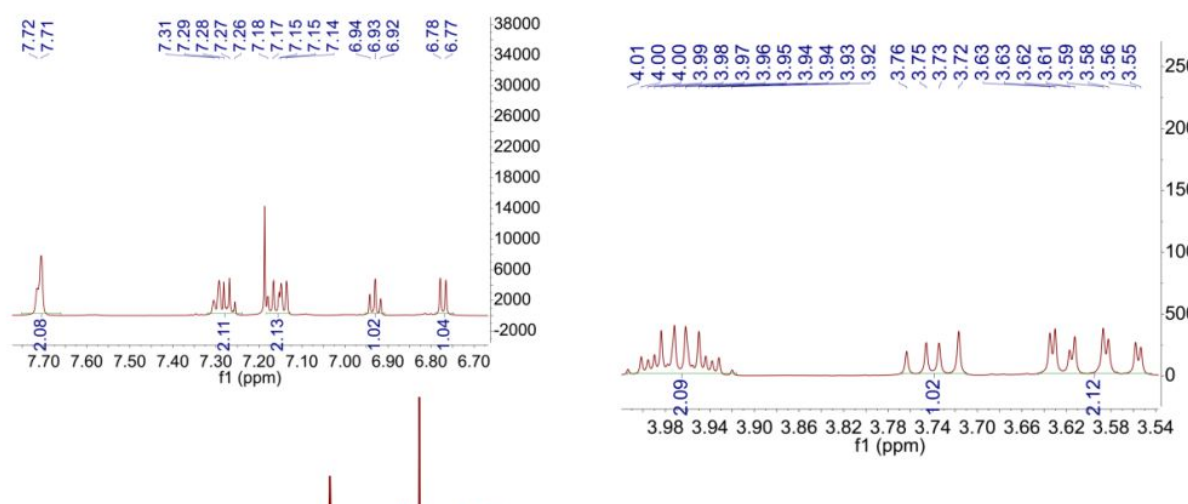

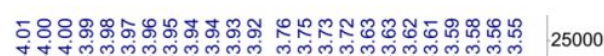

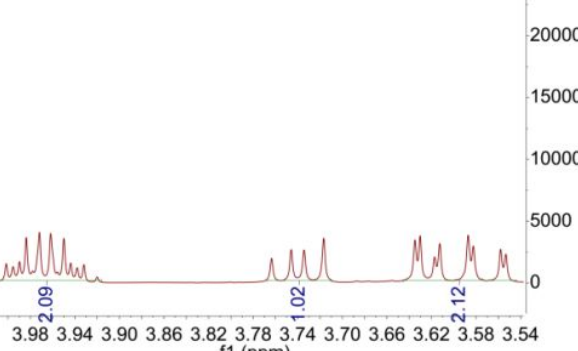

dill

ֻั

ㅇํㅇำ

ले ल

$\begin{array}{lllllllllll}9.0 & 8.5 & 8.0 & 7.5 & 7.0 & 6.5 & 6.0 & 5.5 & 5.0 & 4.5 & 4.0\end{array}$

f1 (ppm) 


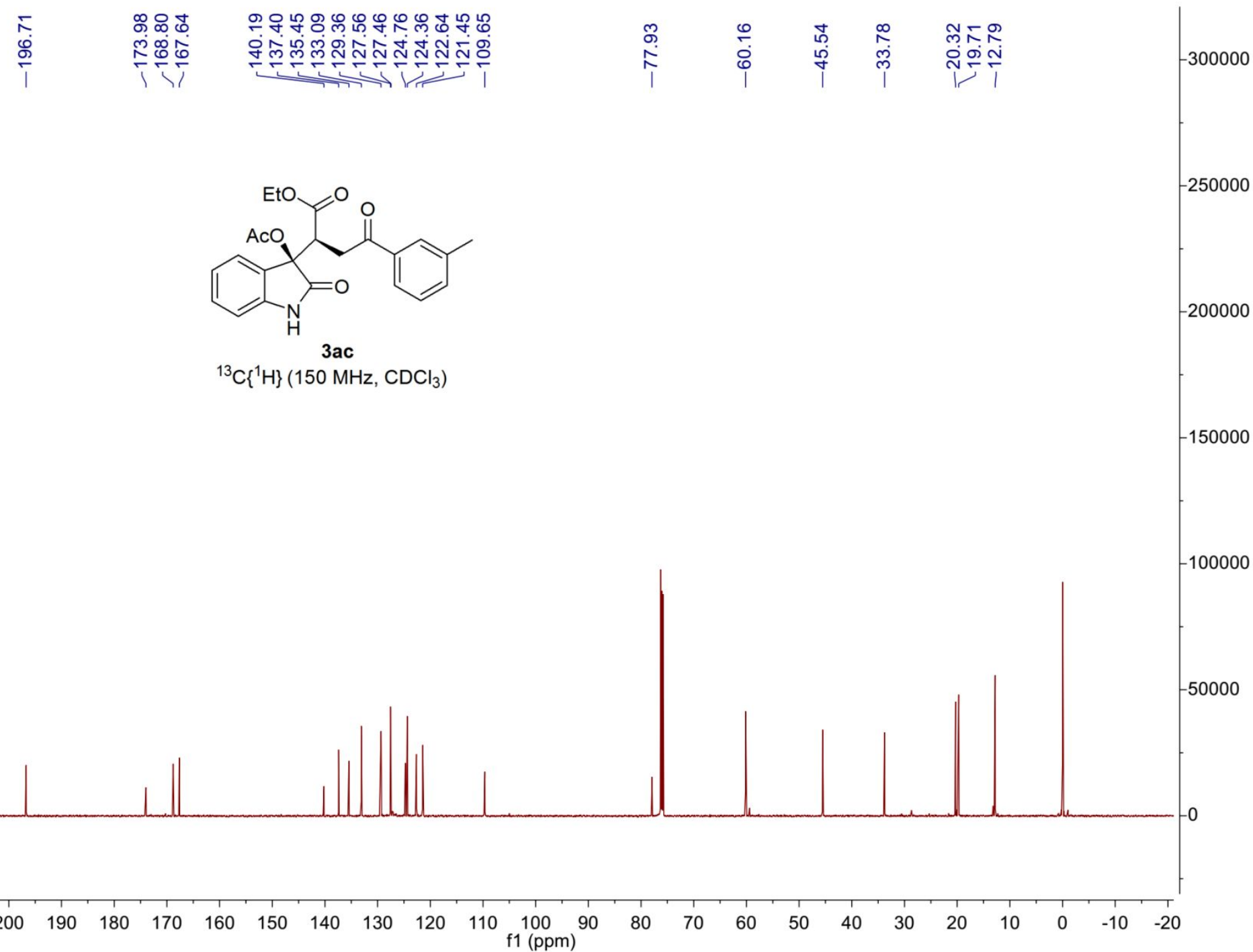




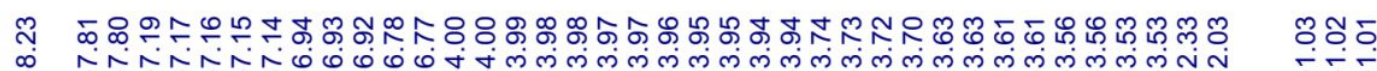

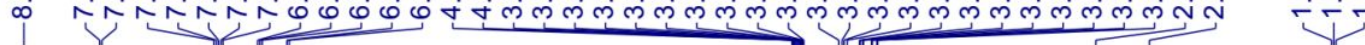

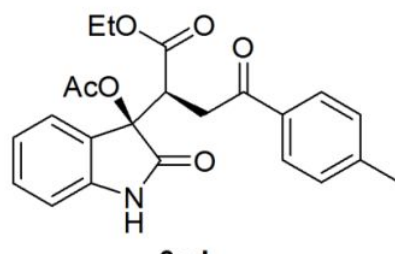

$-35000$

$-30000$

3ad

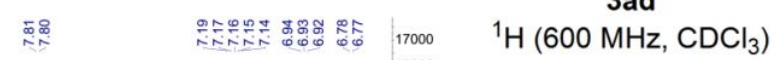

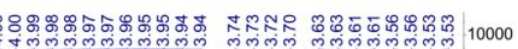

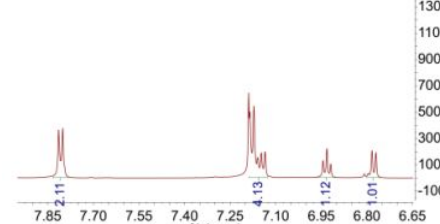

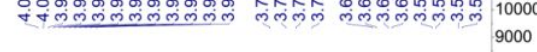

8000

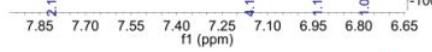
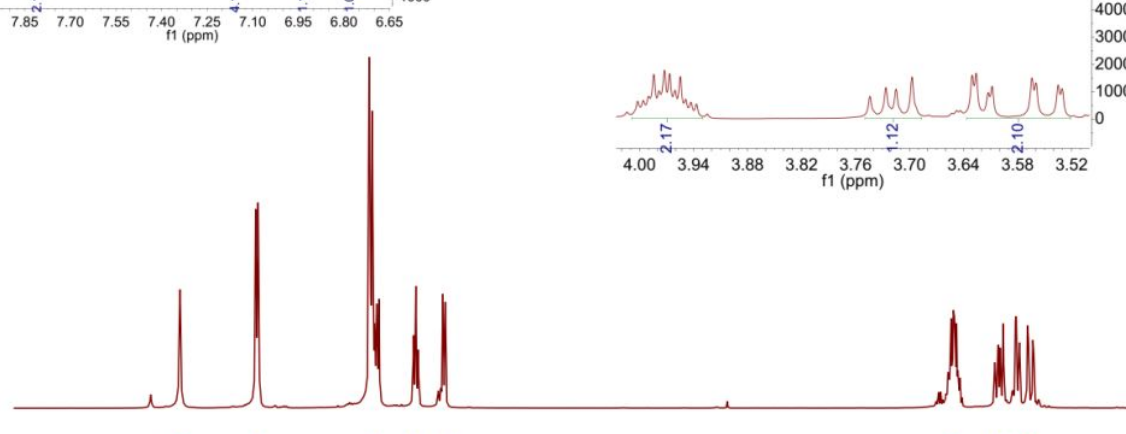

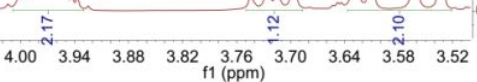

$-20000$

$-15000$

10000

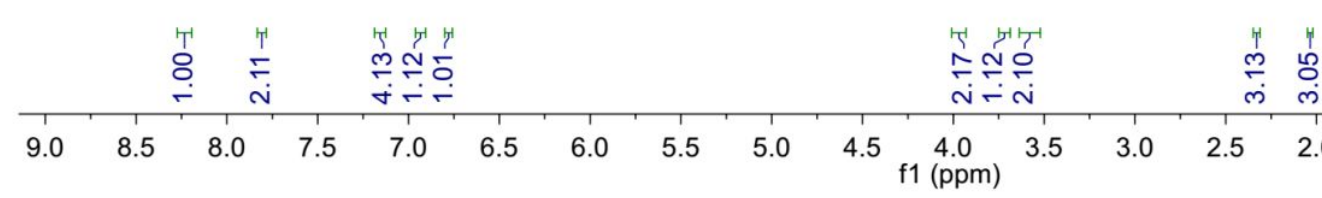

ᄂ

in

$-5000$ 


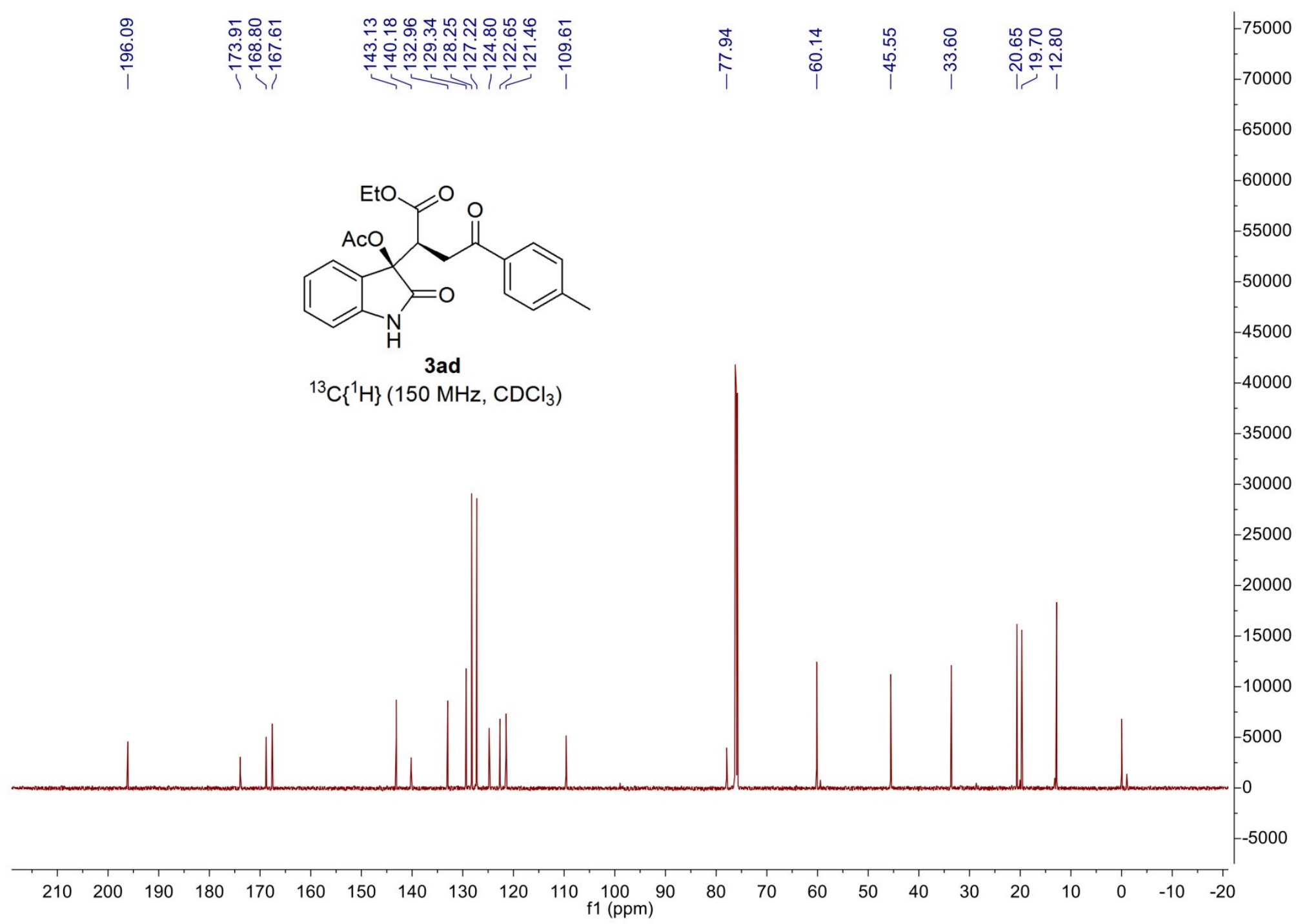




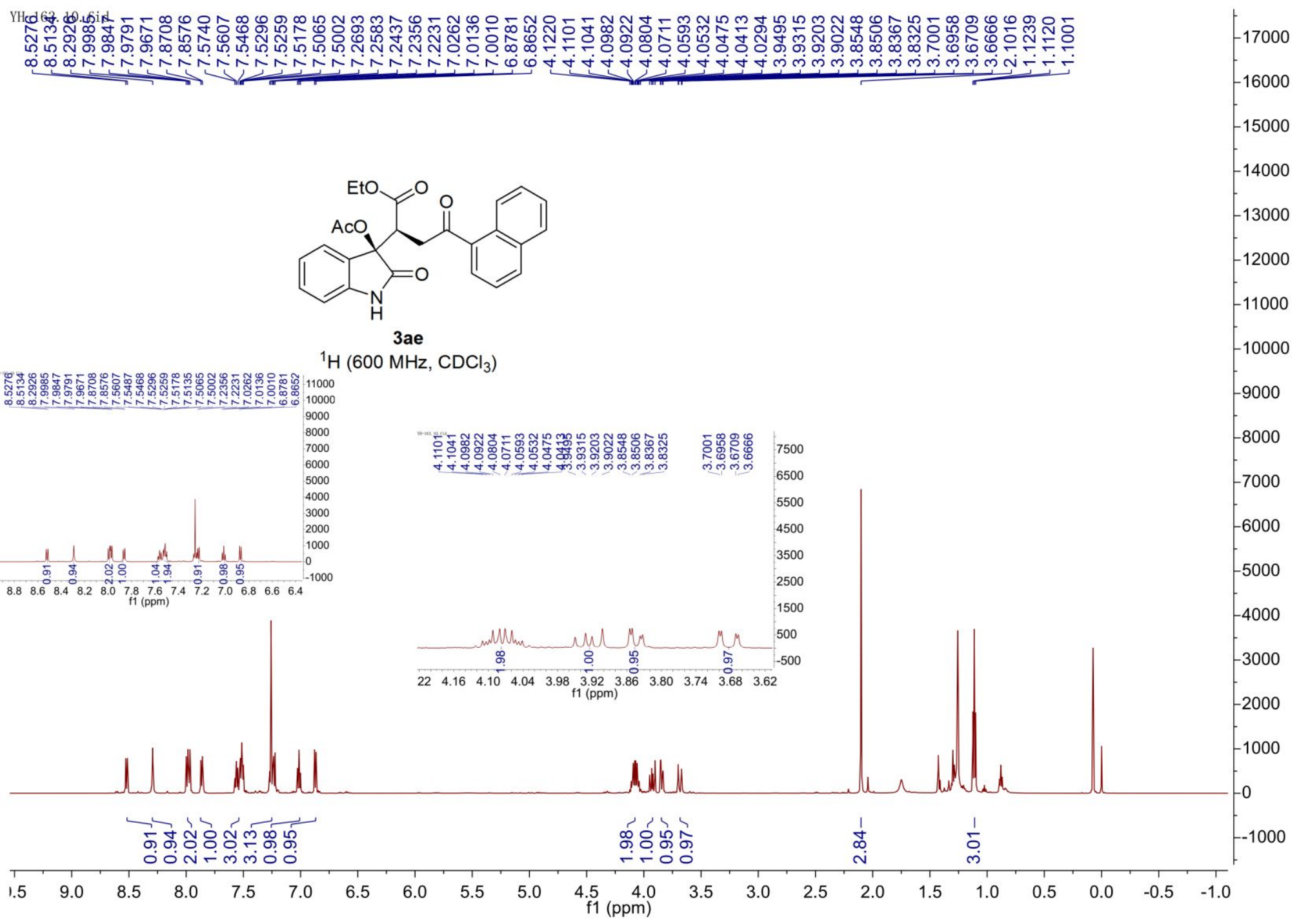




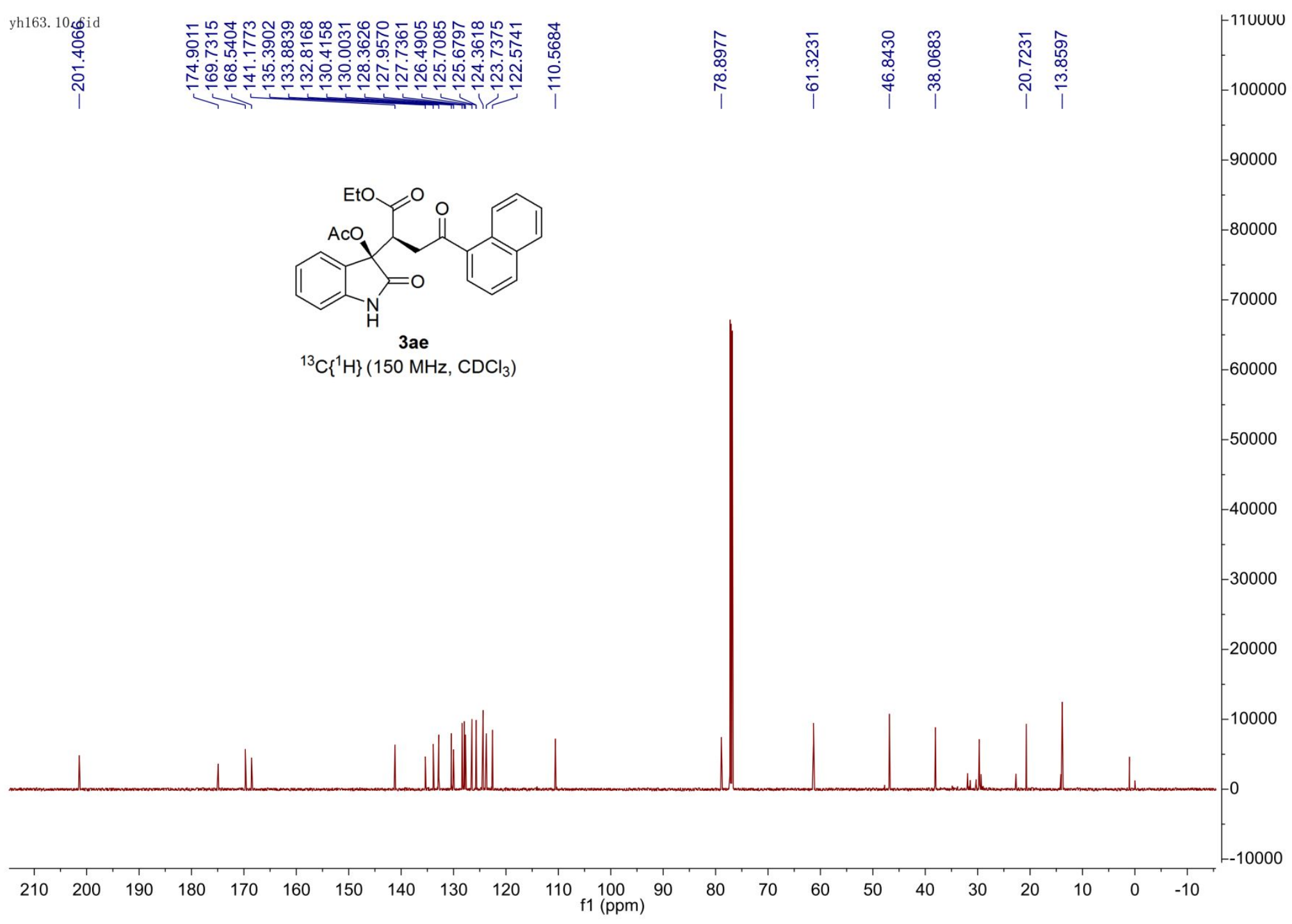




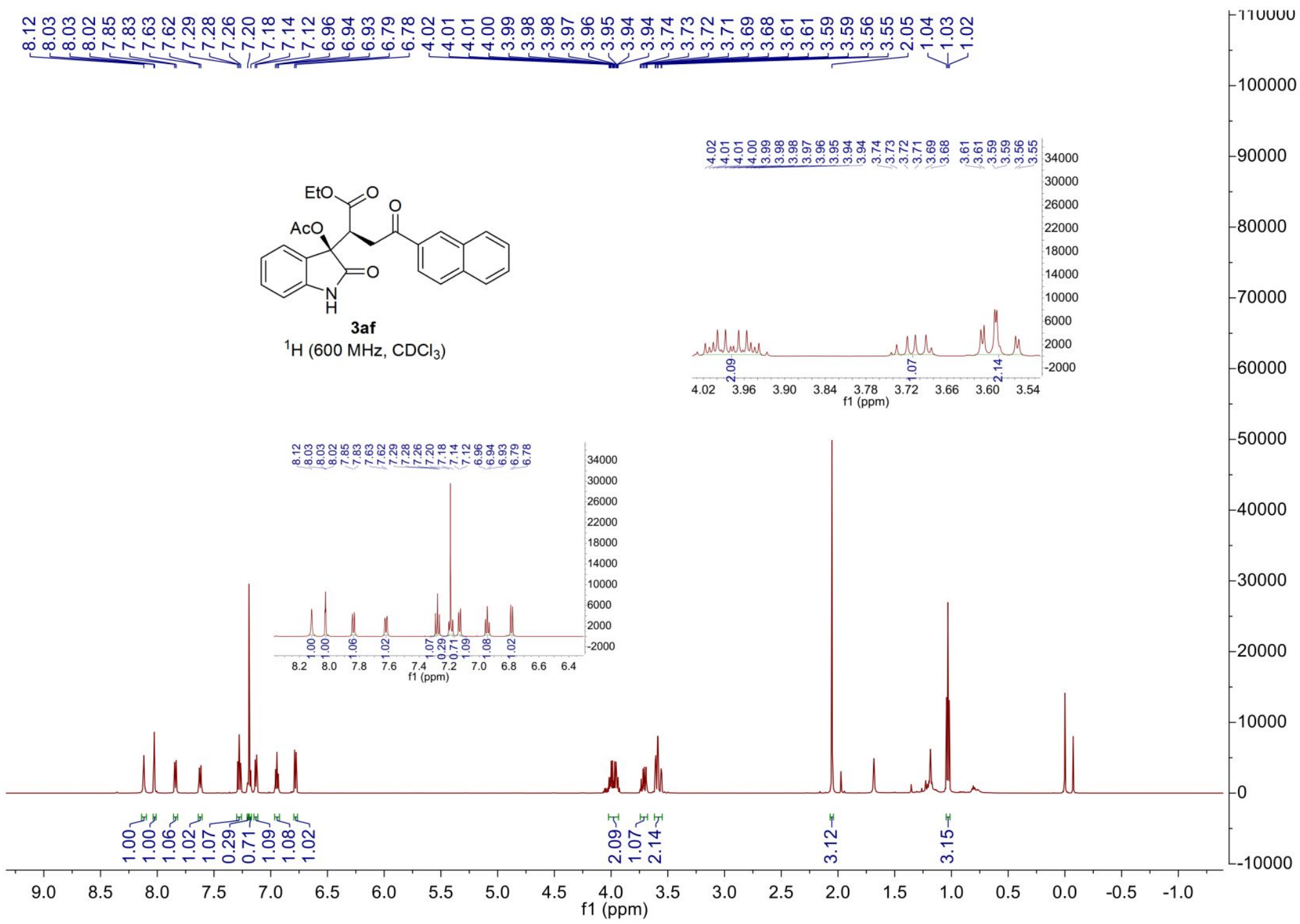




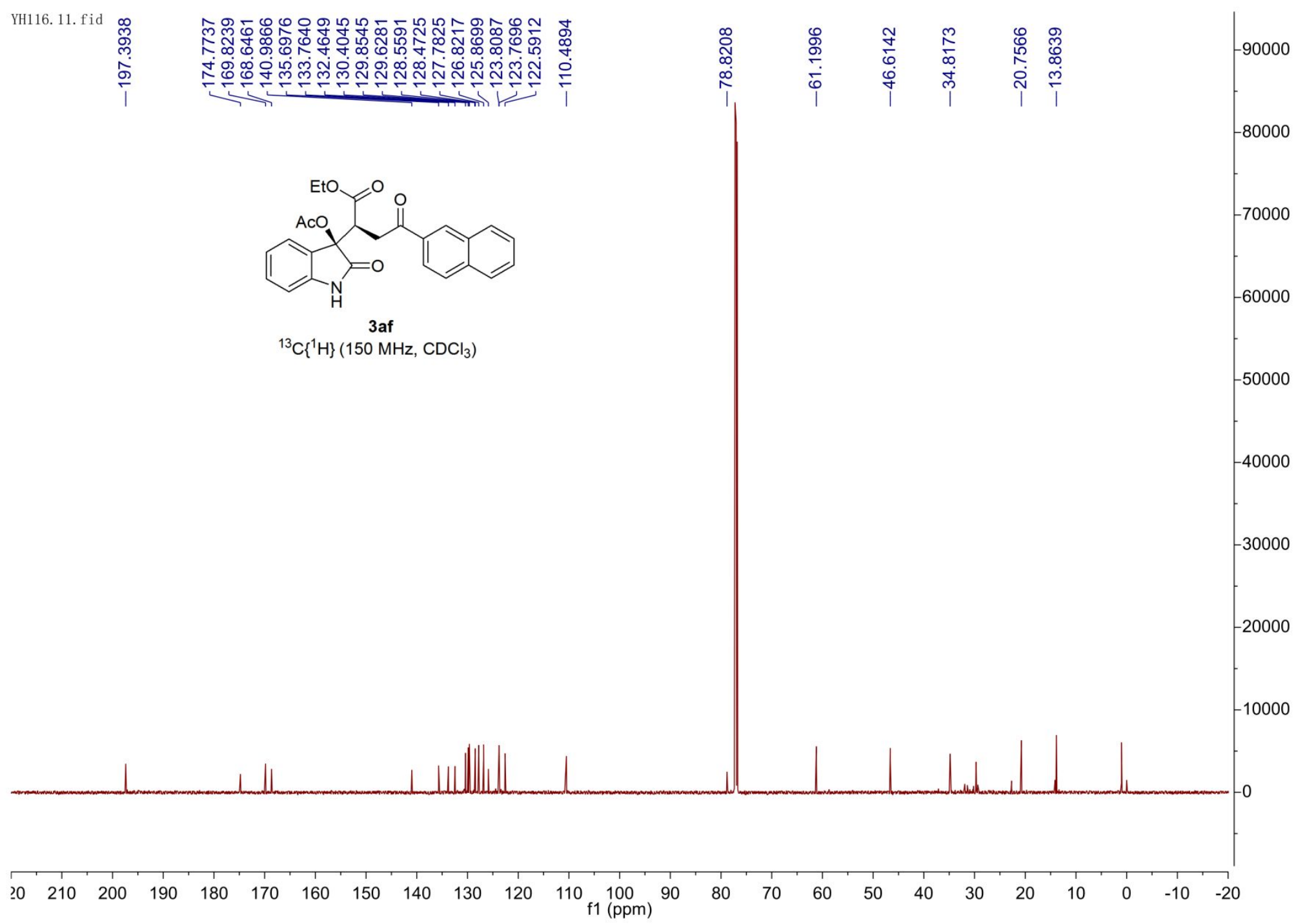




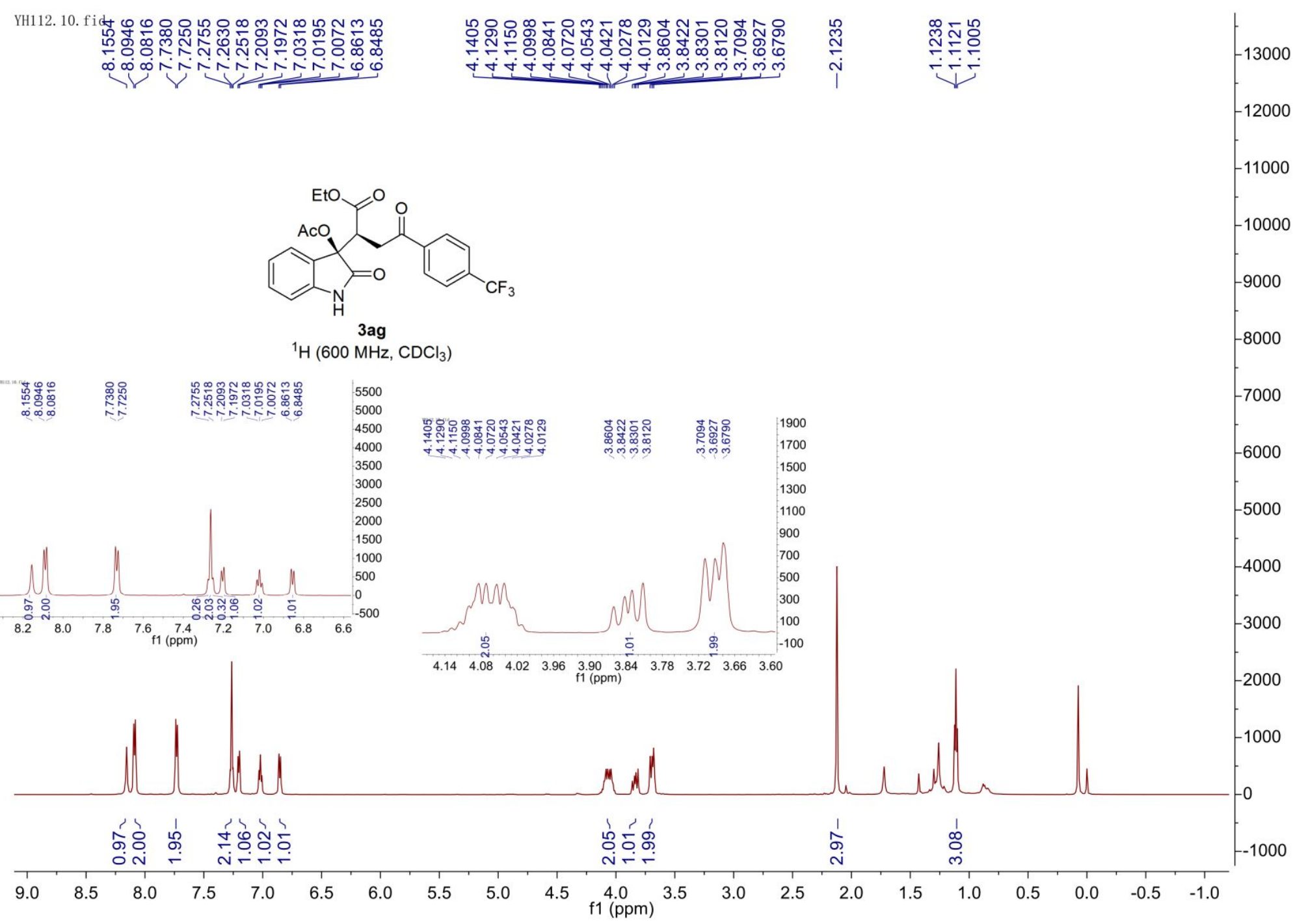




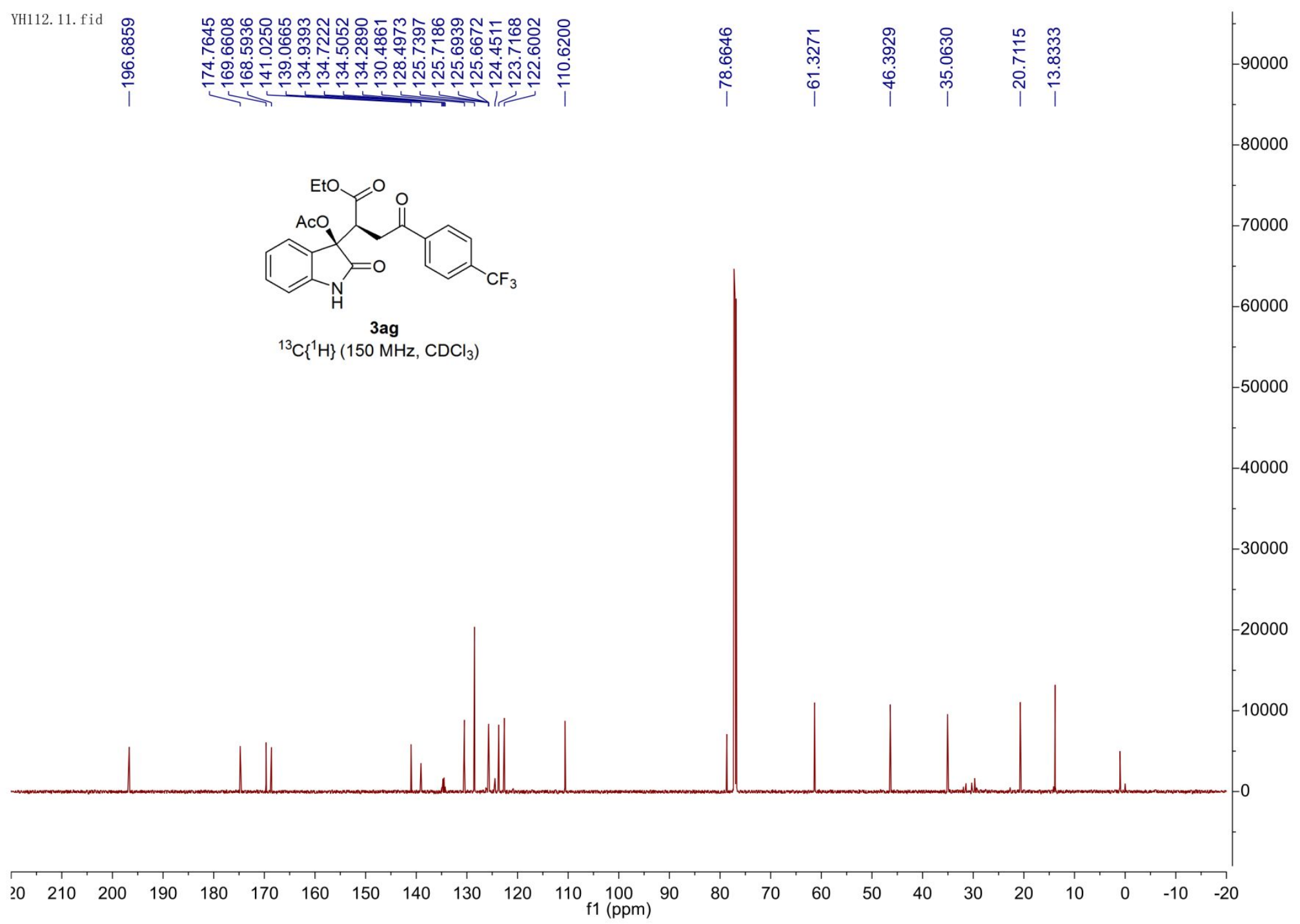




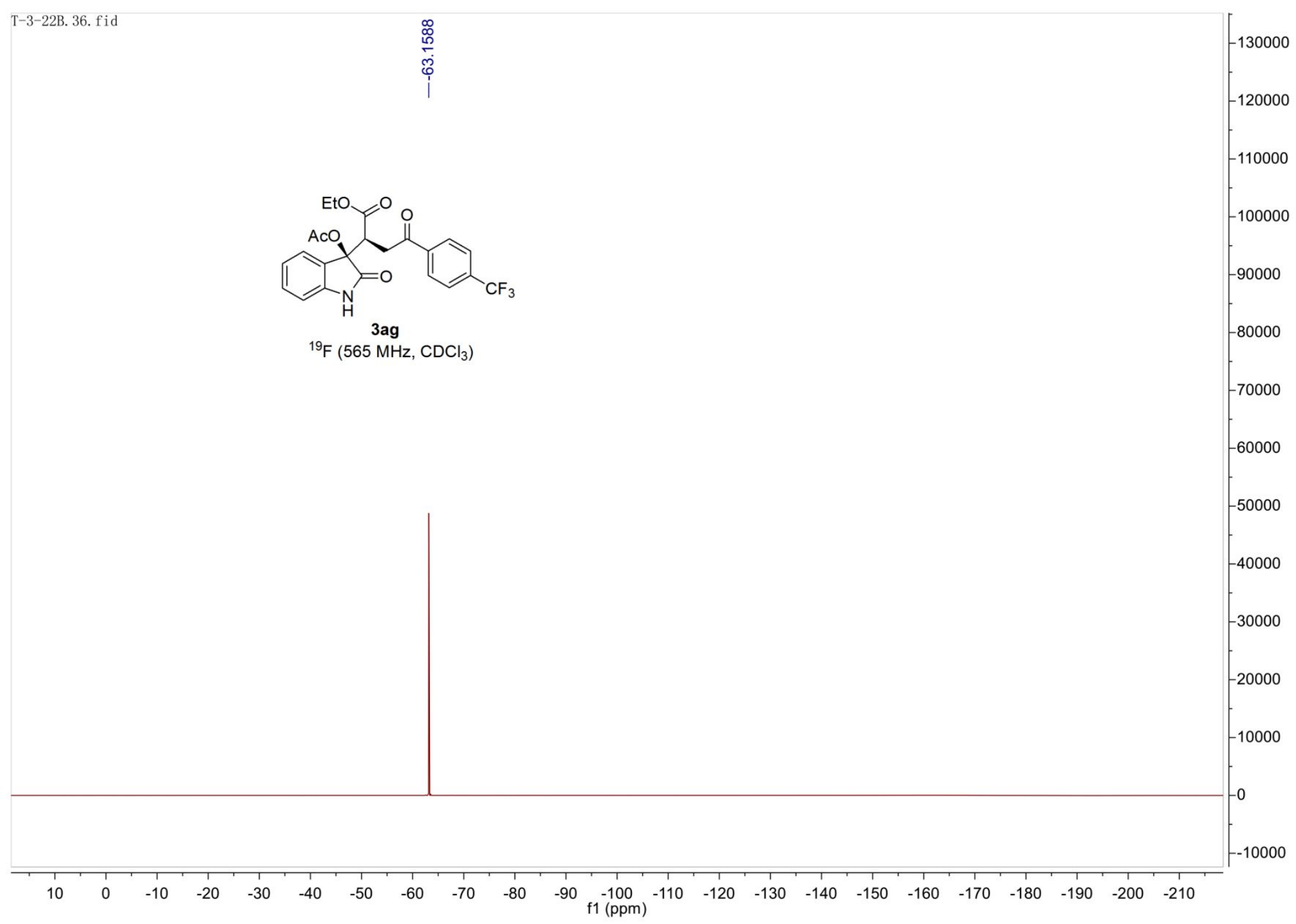




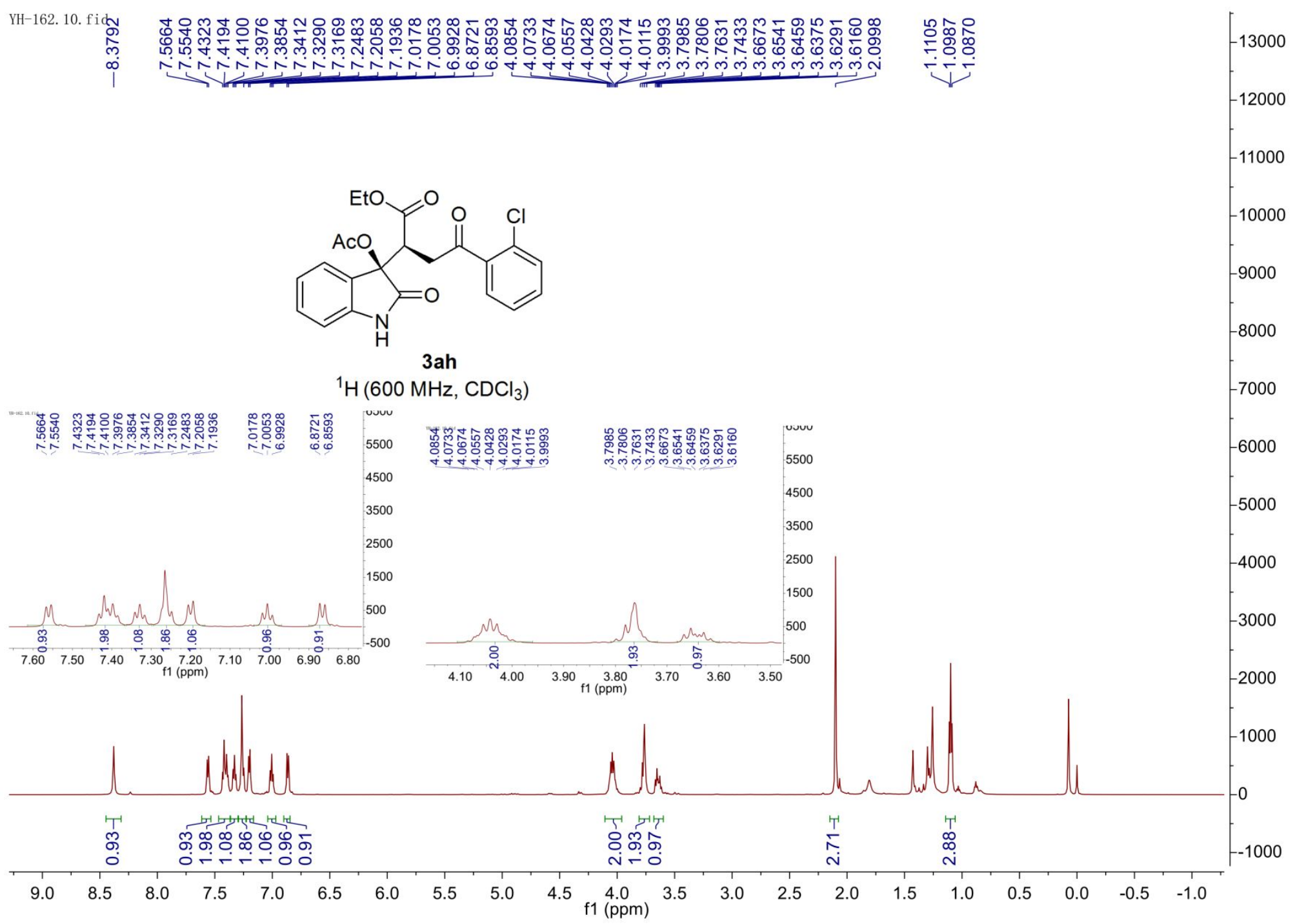




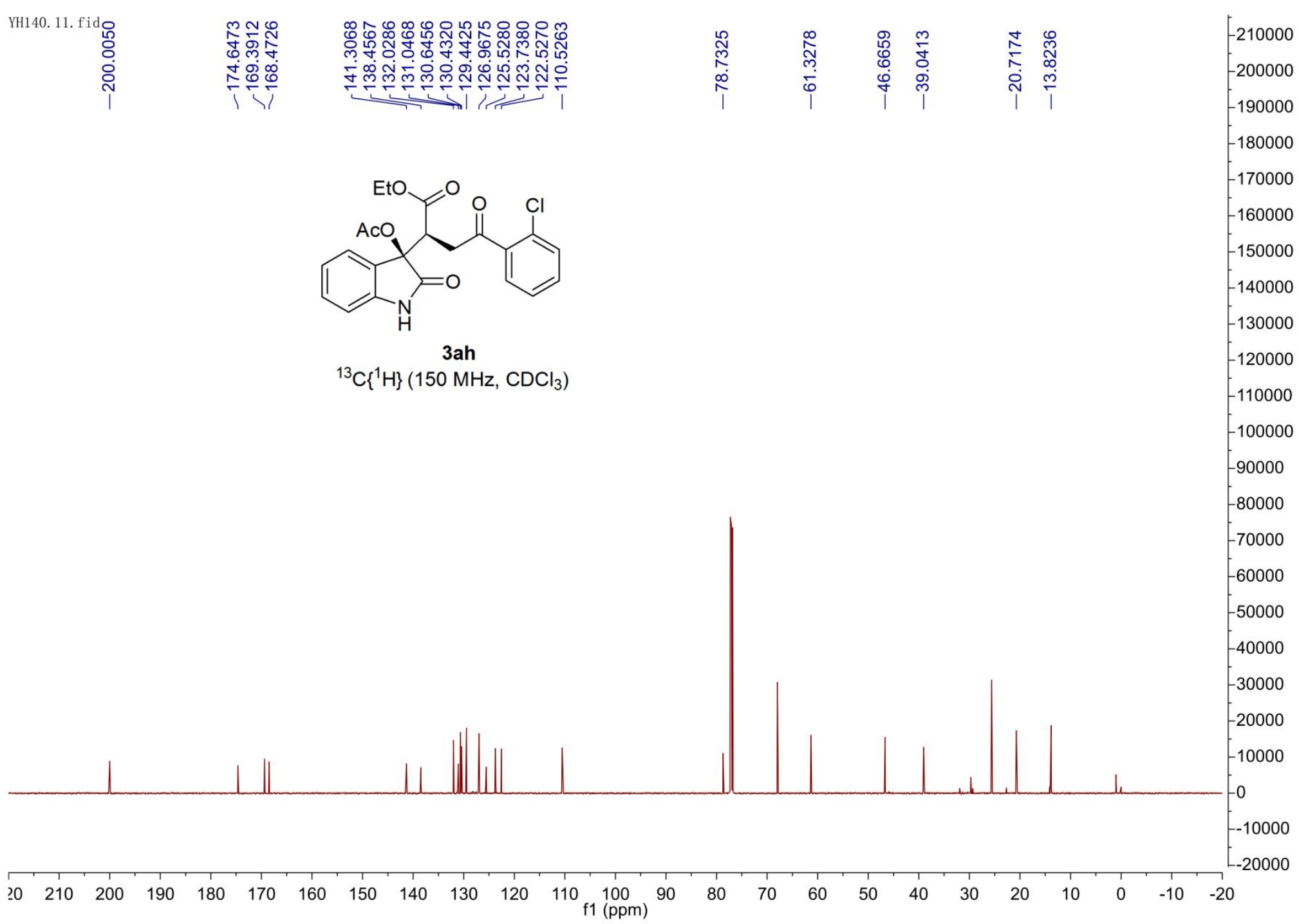




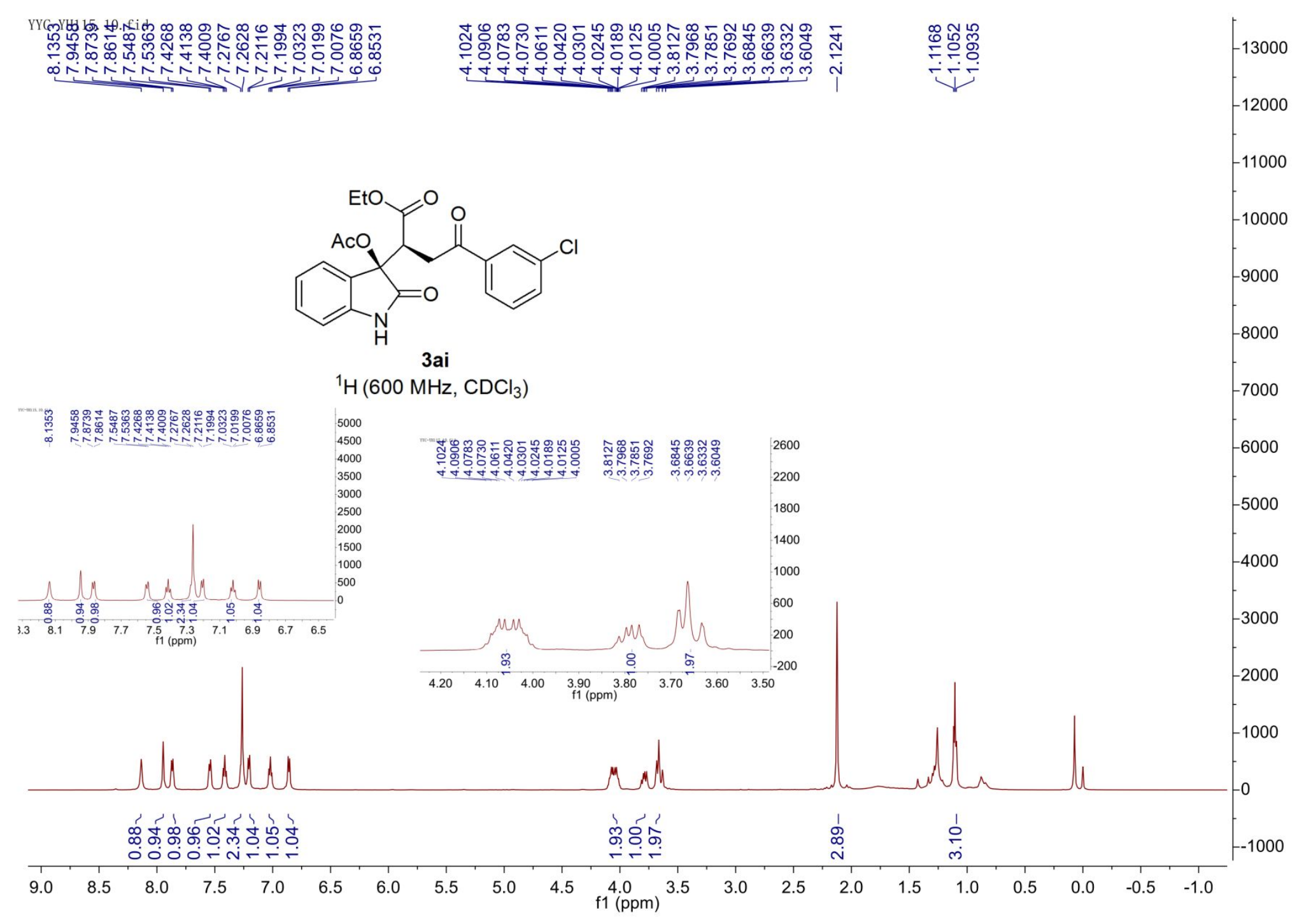




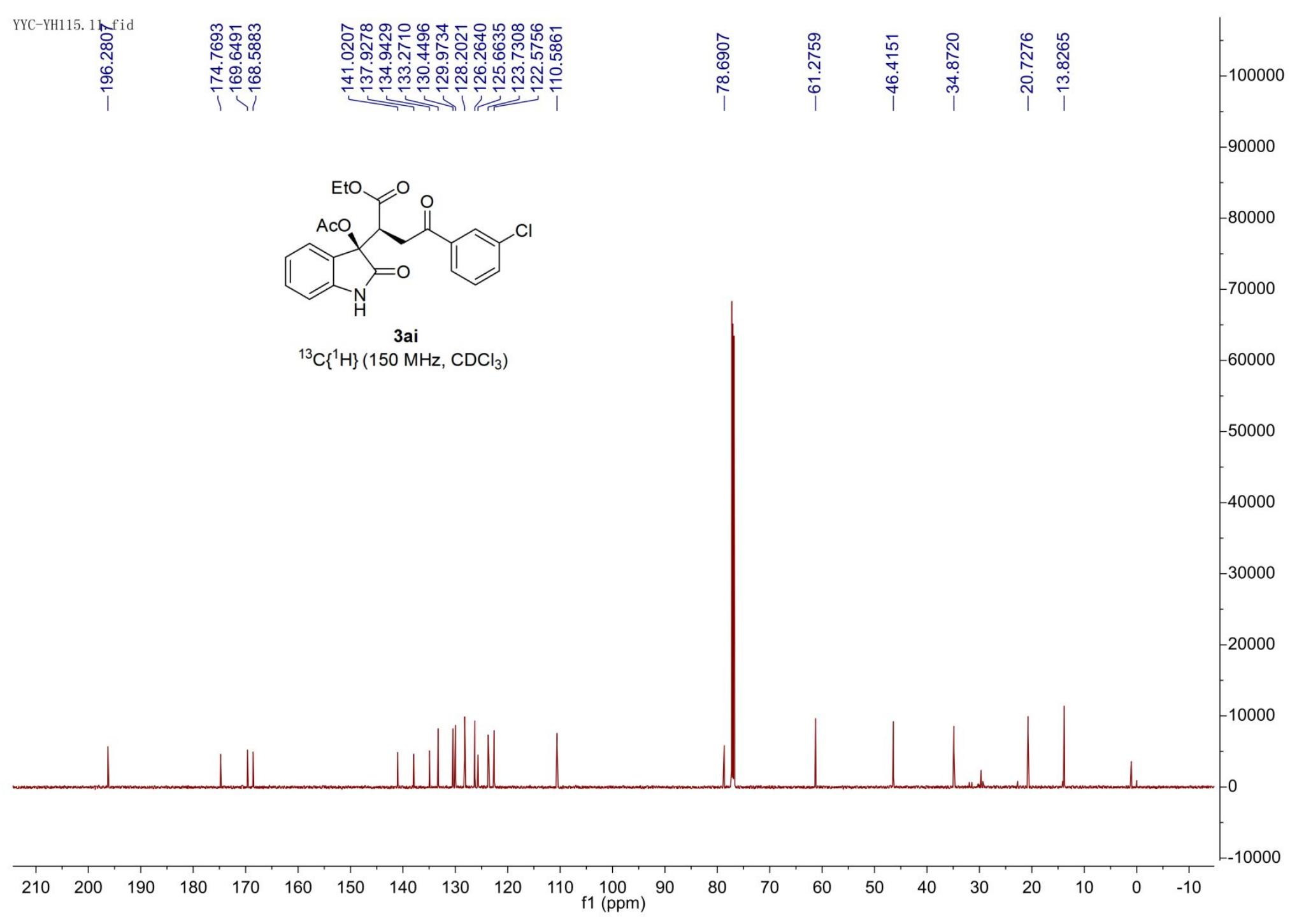




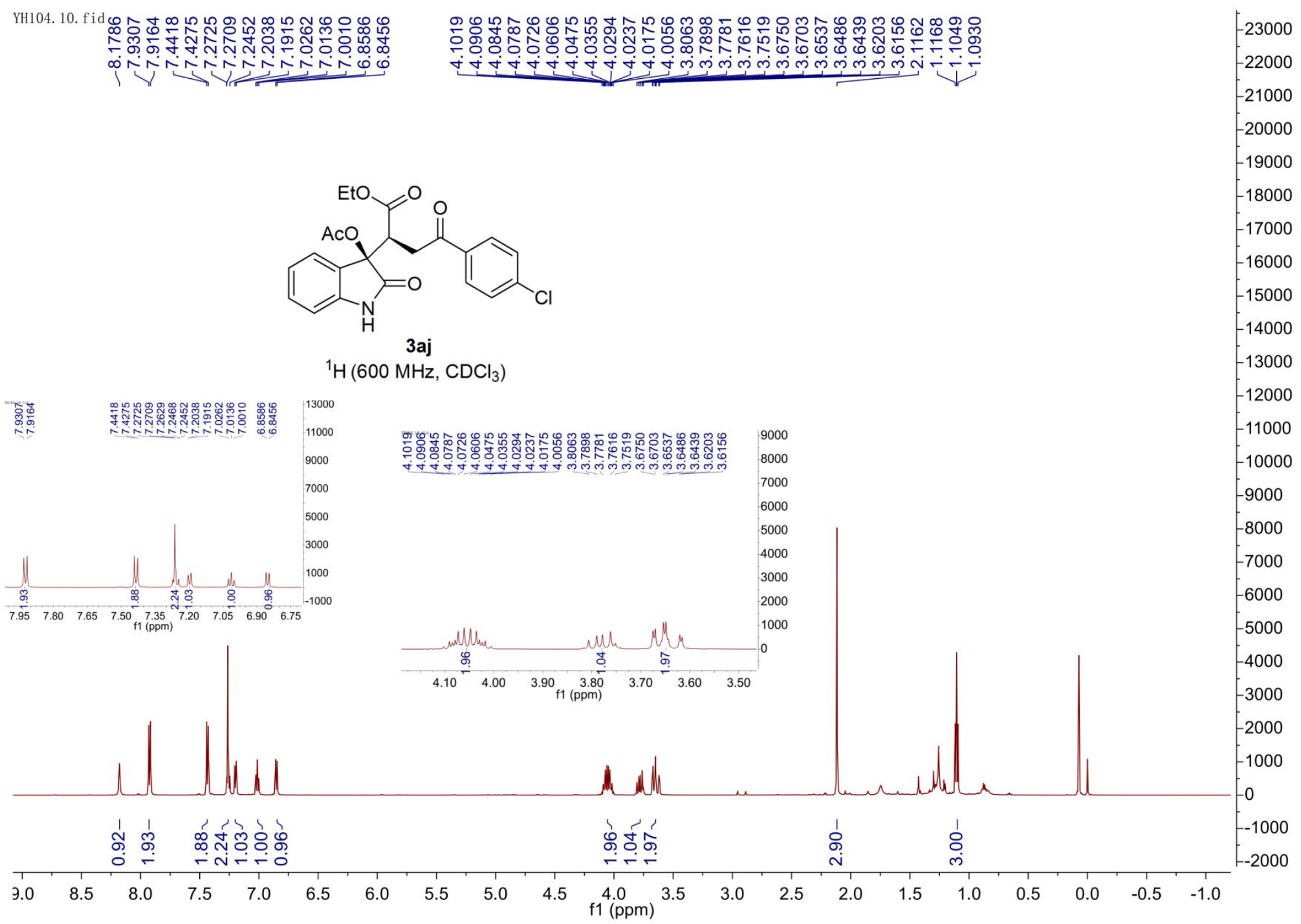




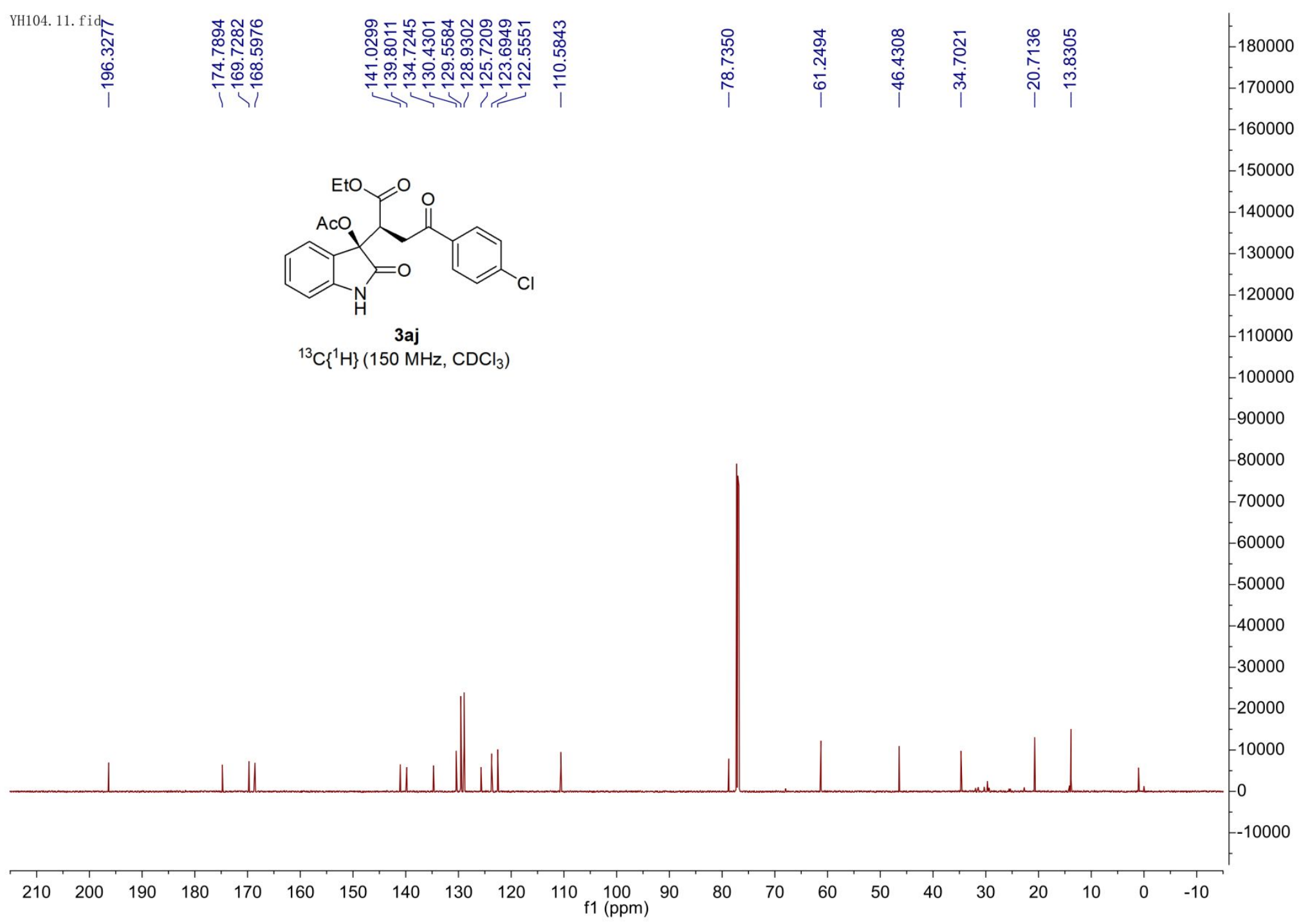




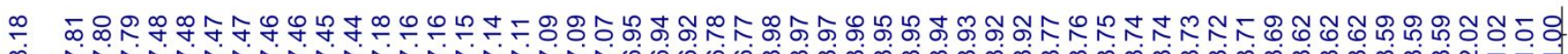

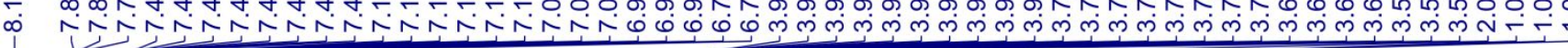

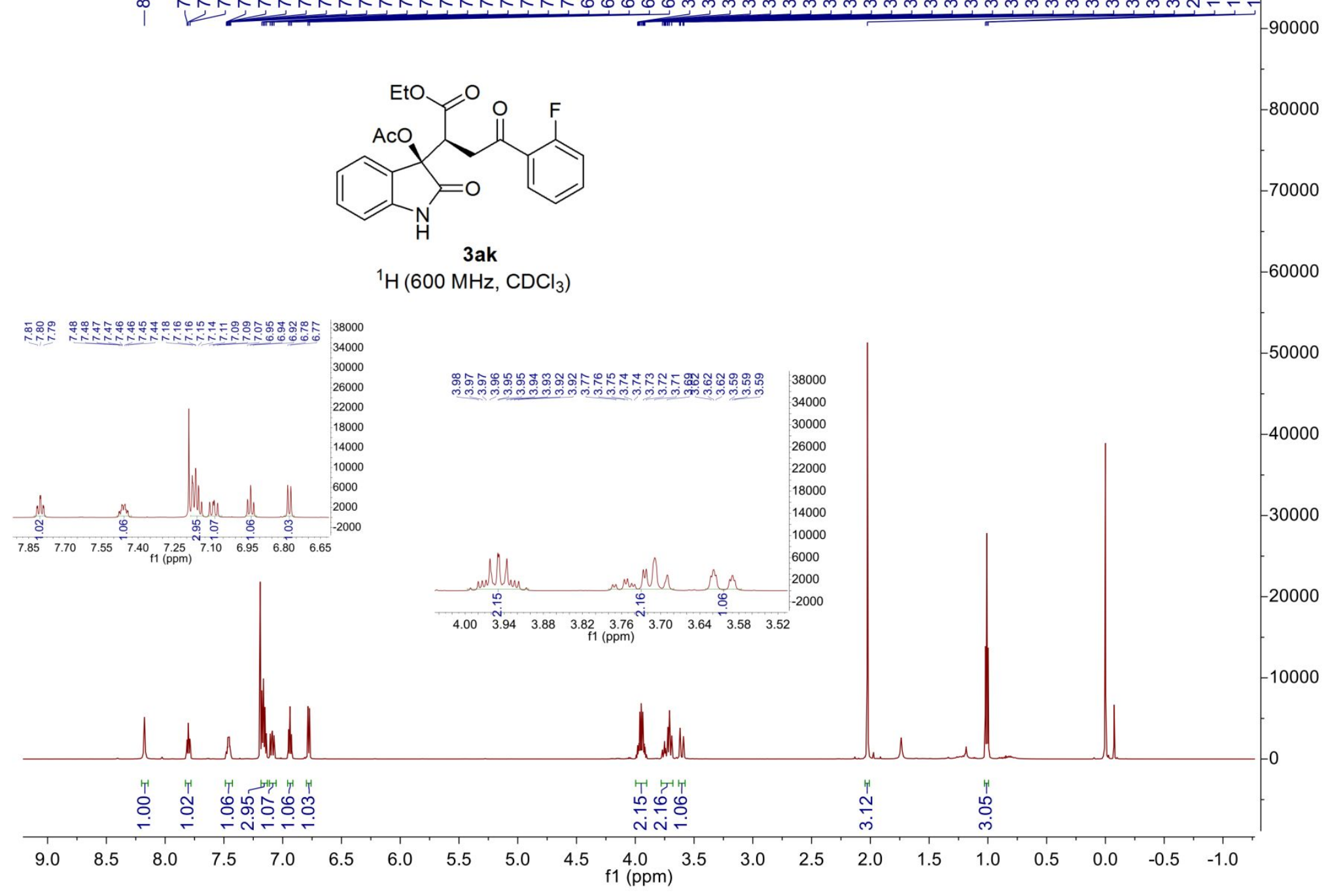




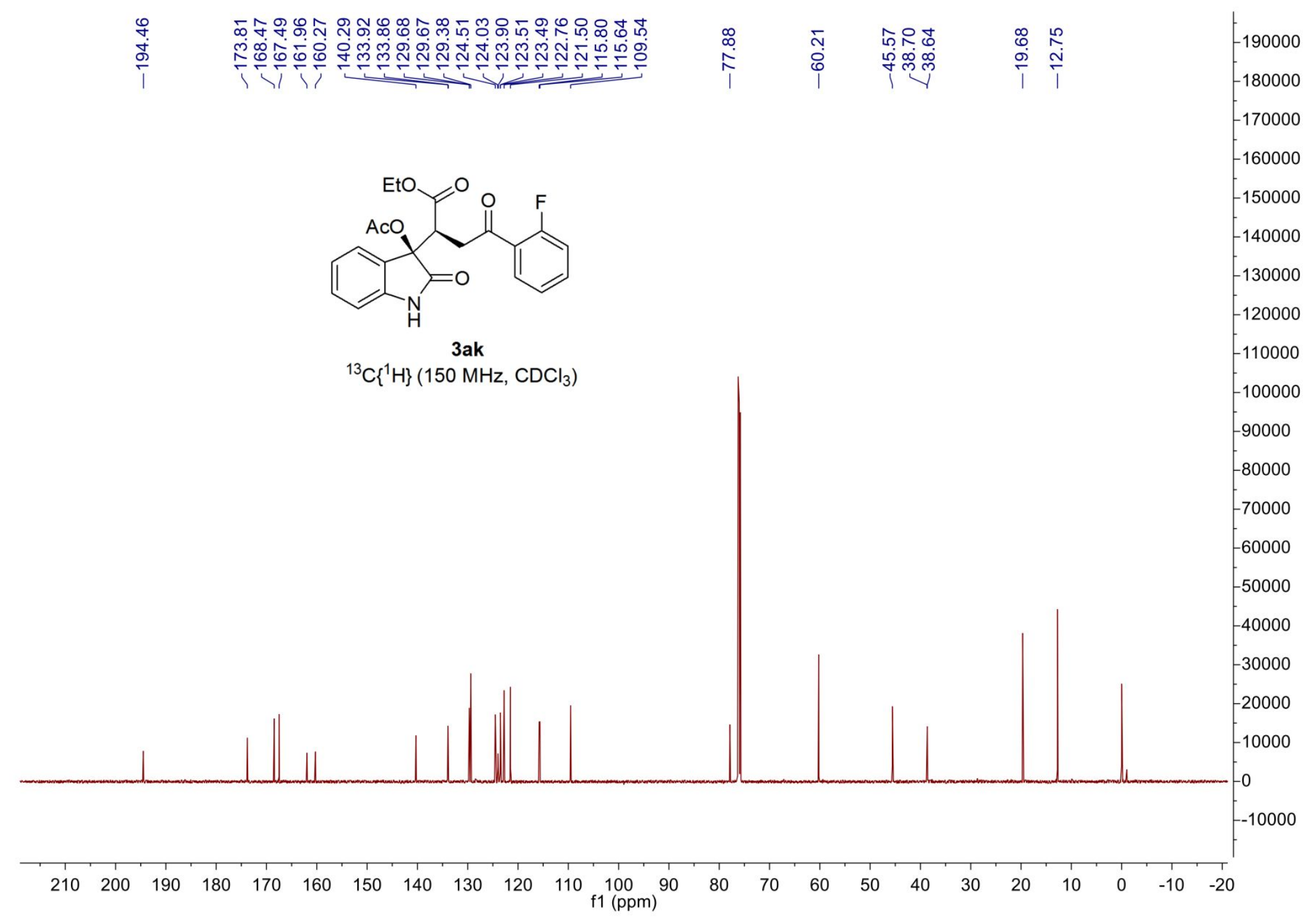




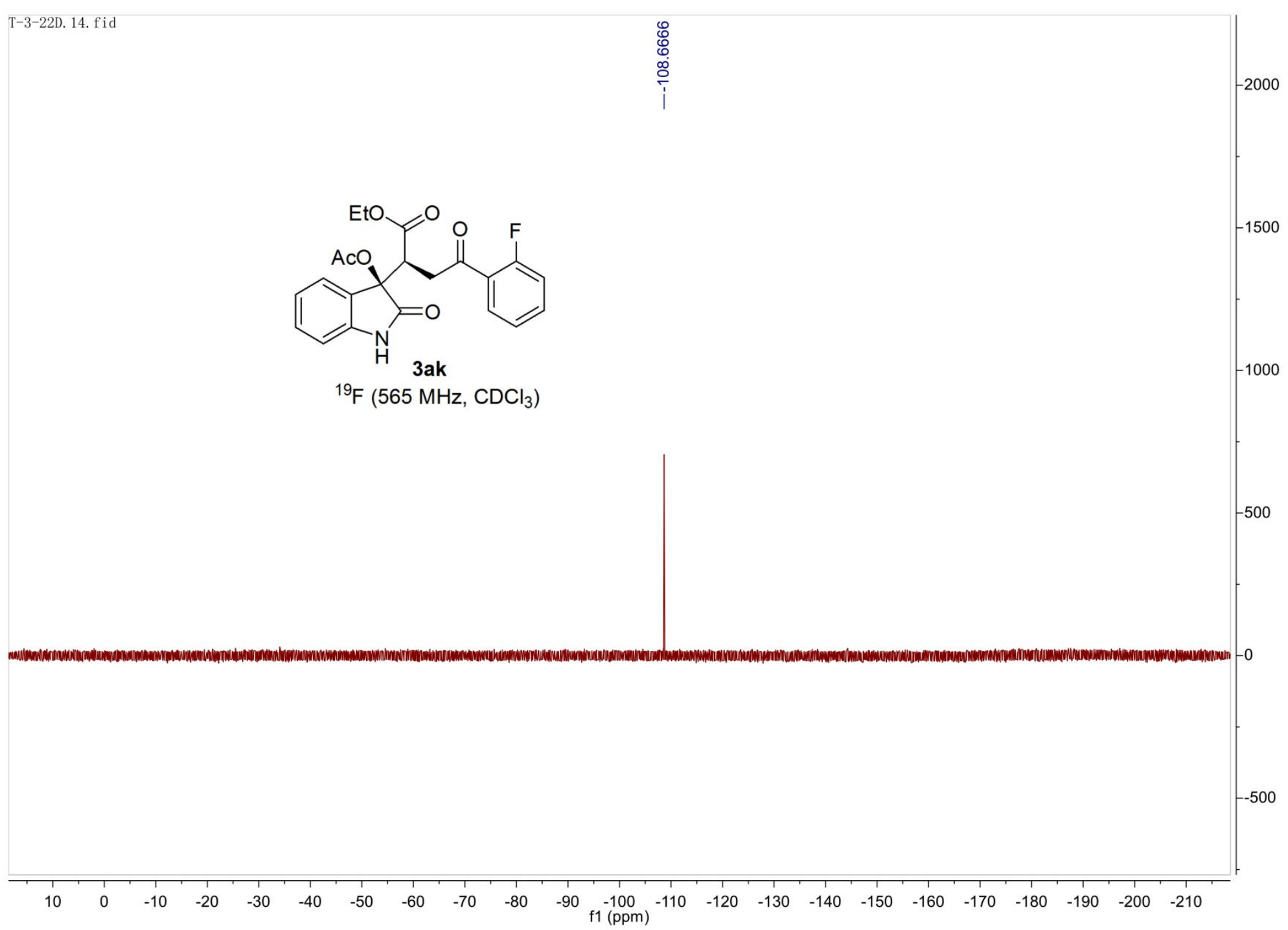




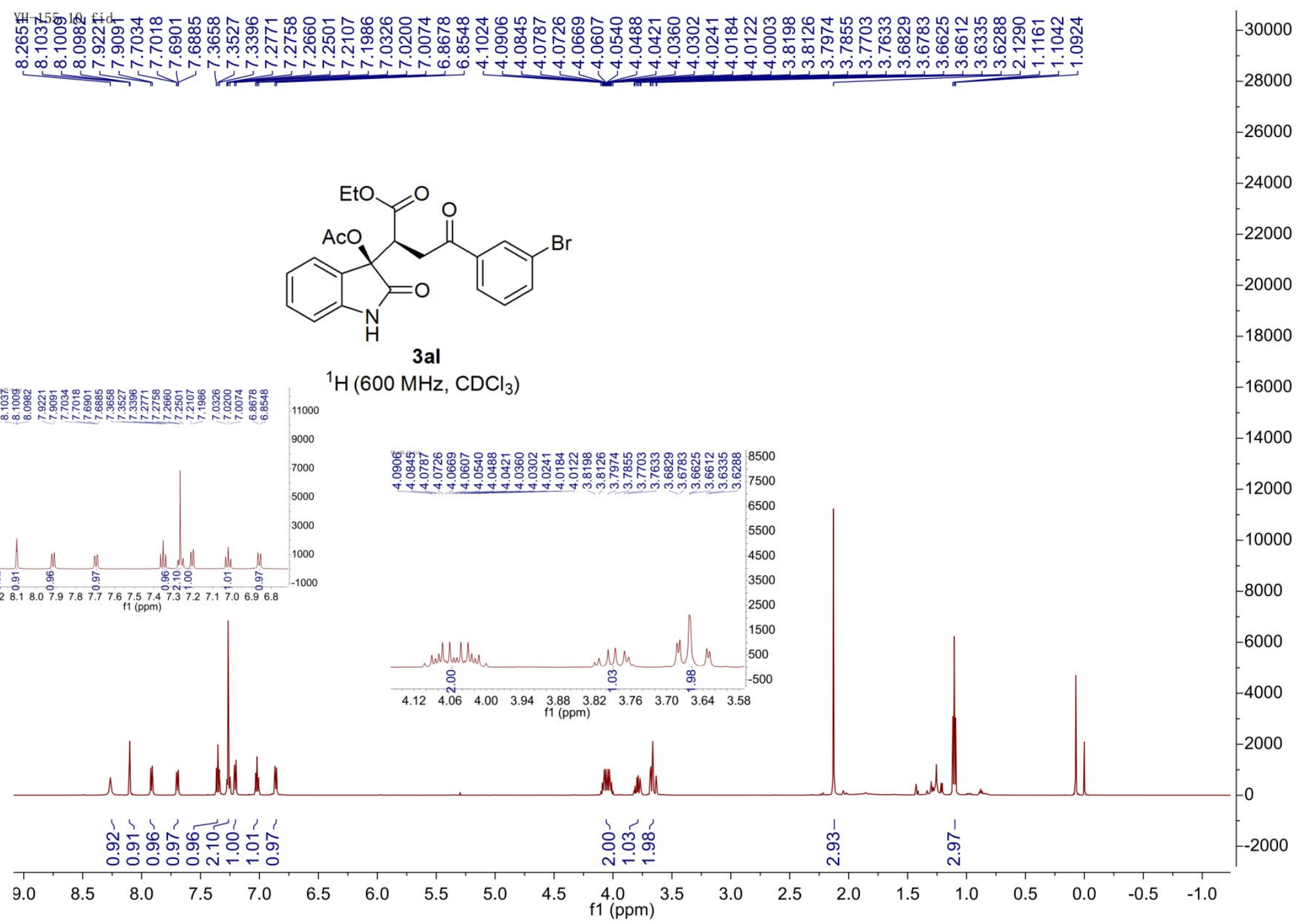




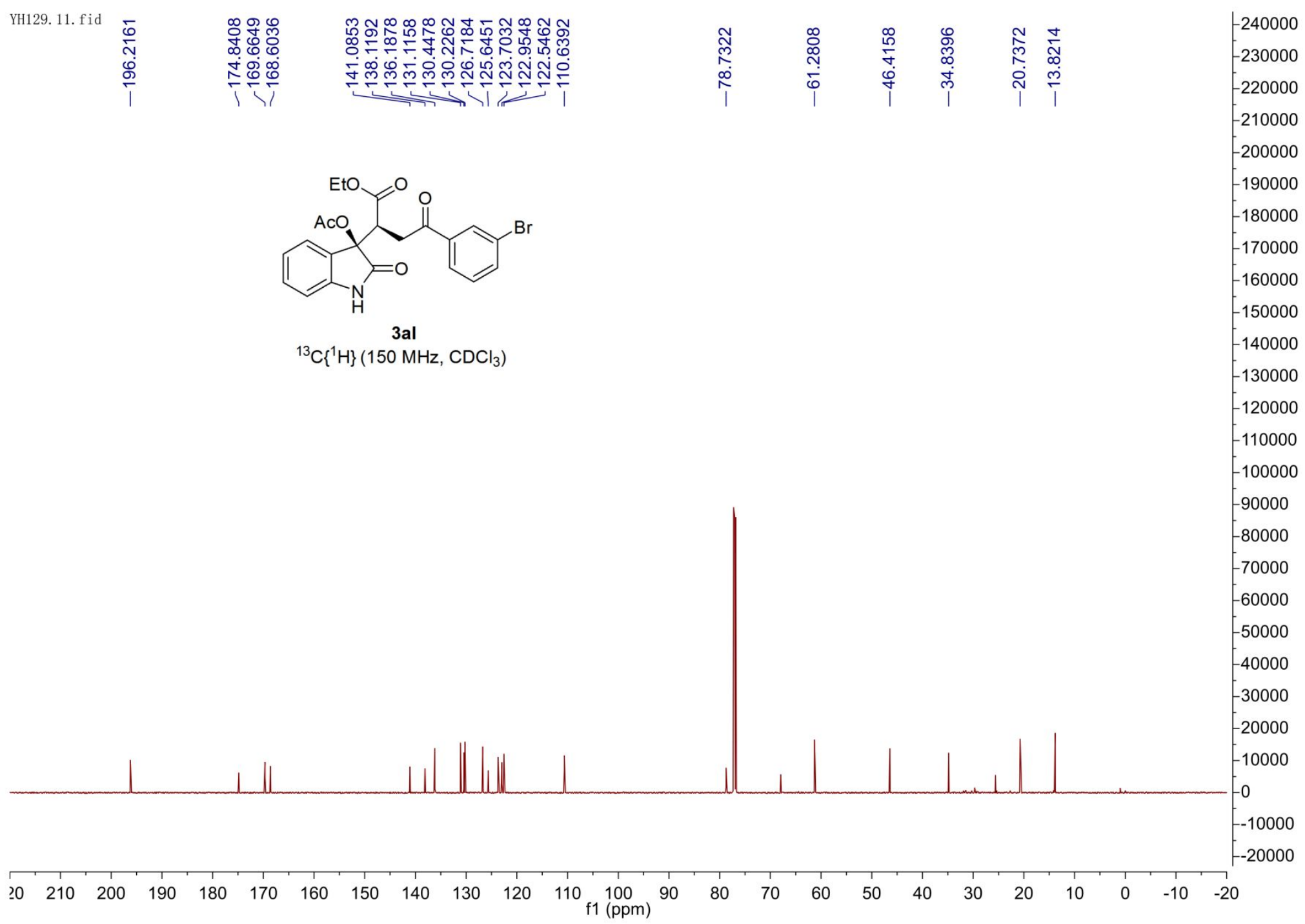




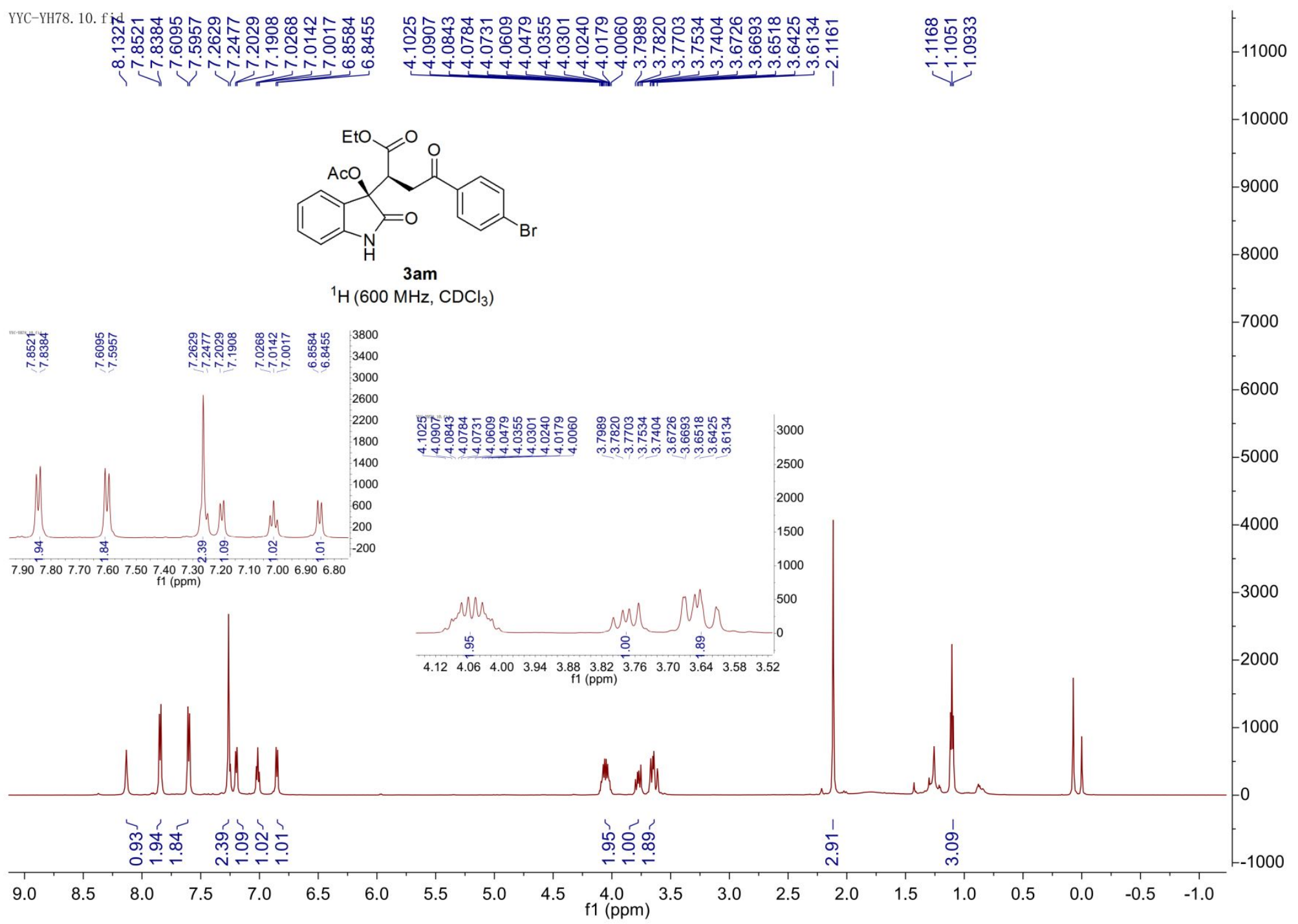




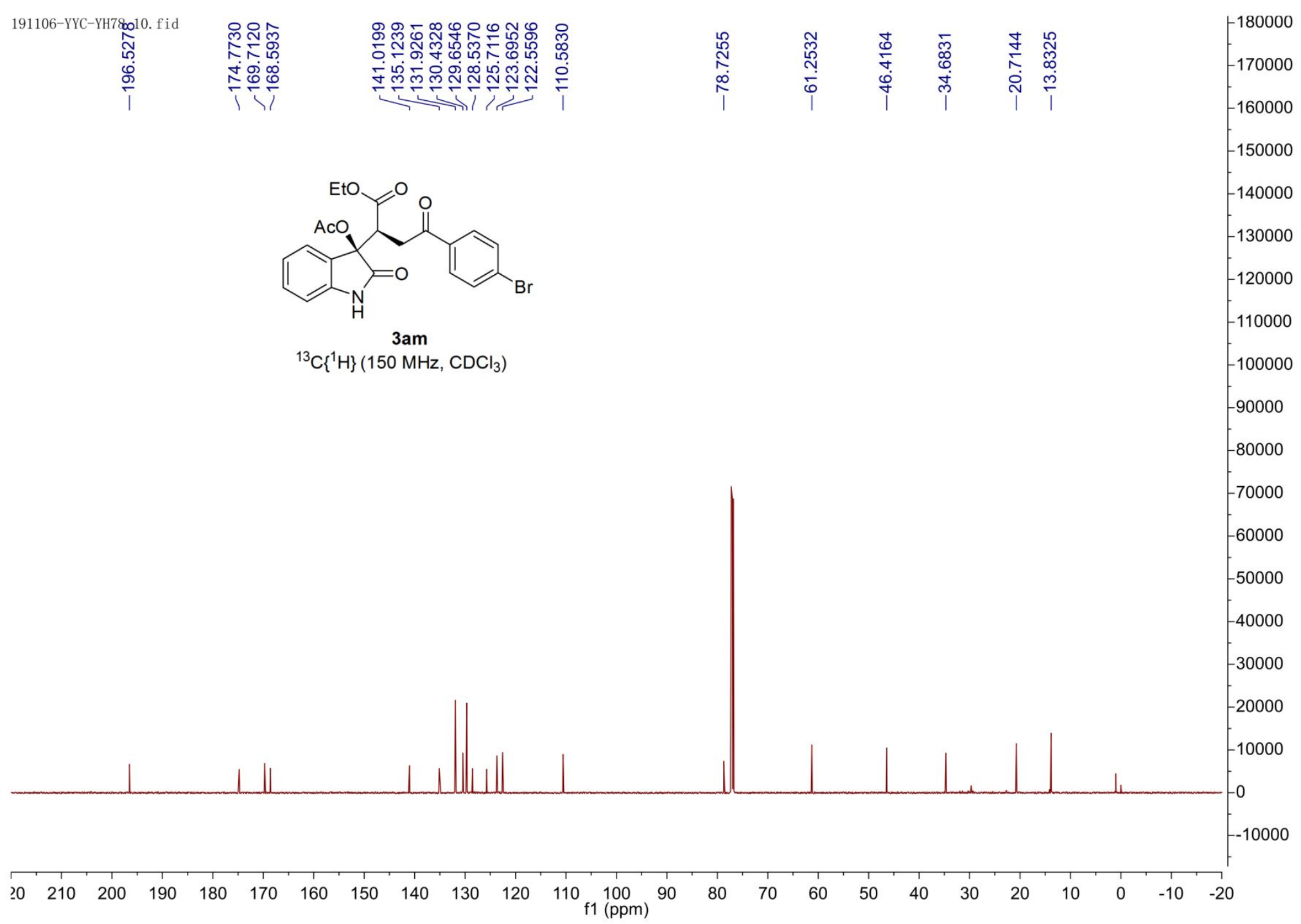




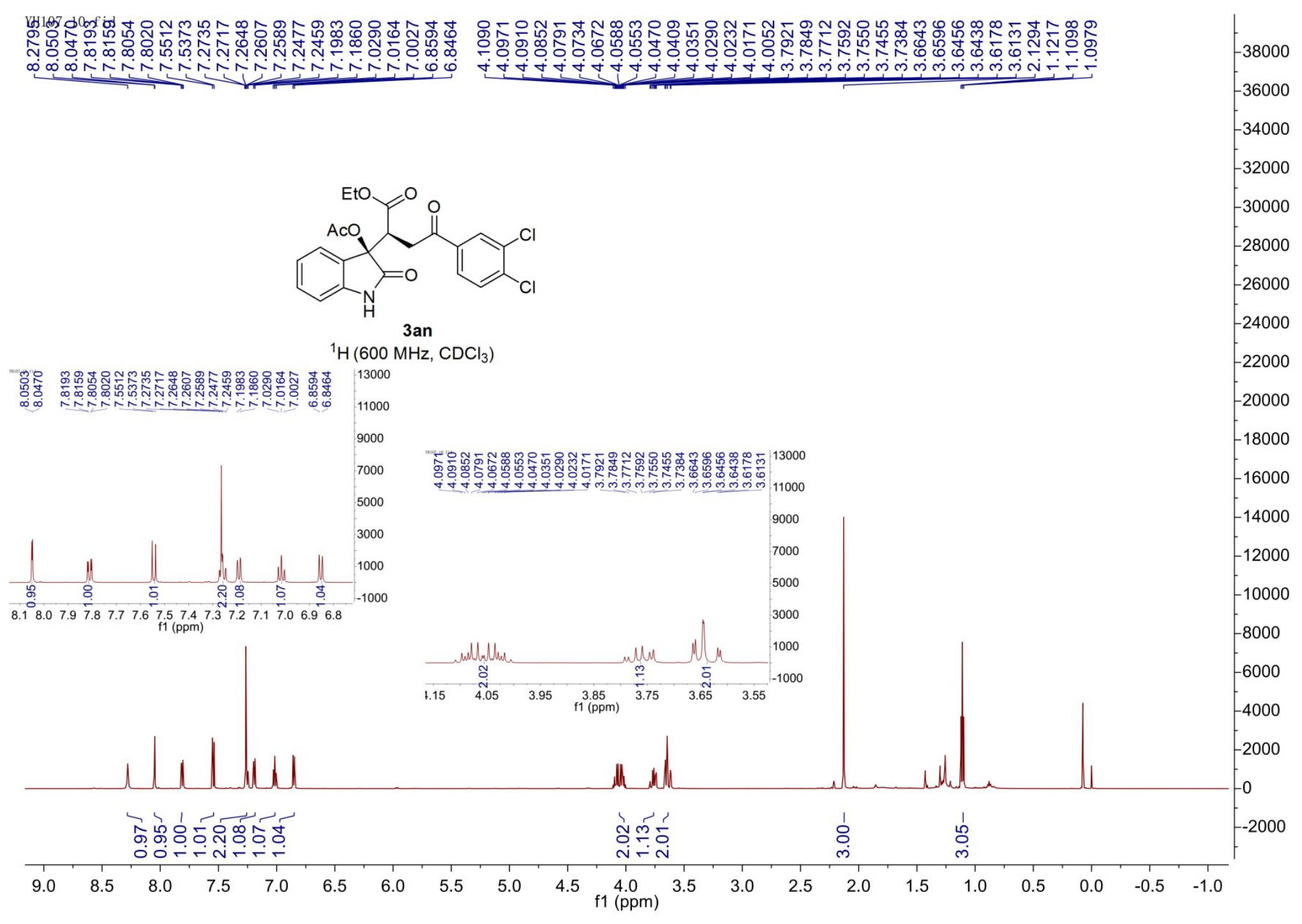




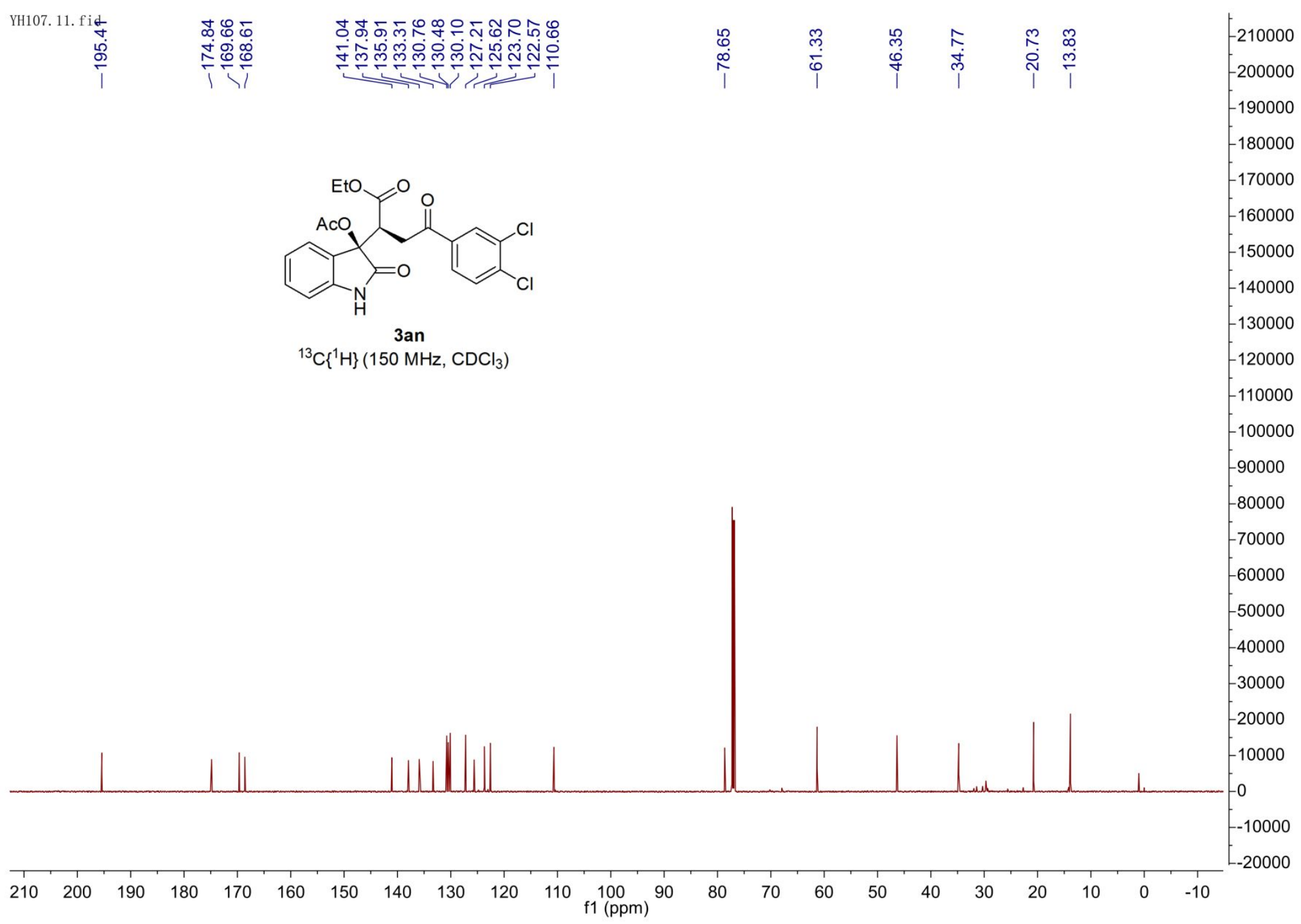




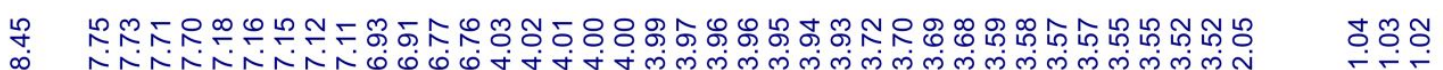

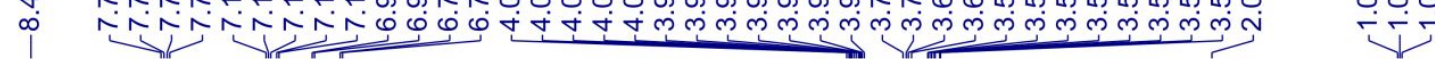

38000

36000

34000

$-32000$

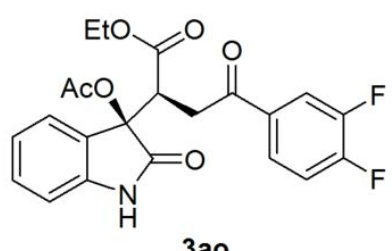

3 ao

${ }^{1} \mathrm{H}\left(600 \mathrm{MHz}, \mathrm{CDCl}_{3}\right)$

$-30000$

$-28000$

$-26000$

$-24000$

$-22000$

$-20000$
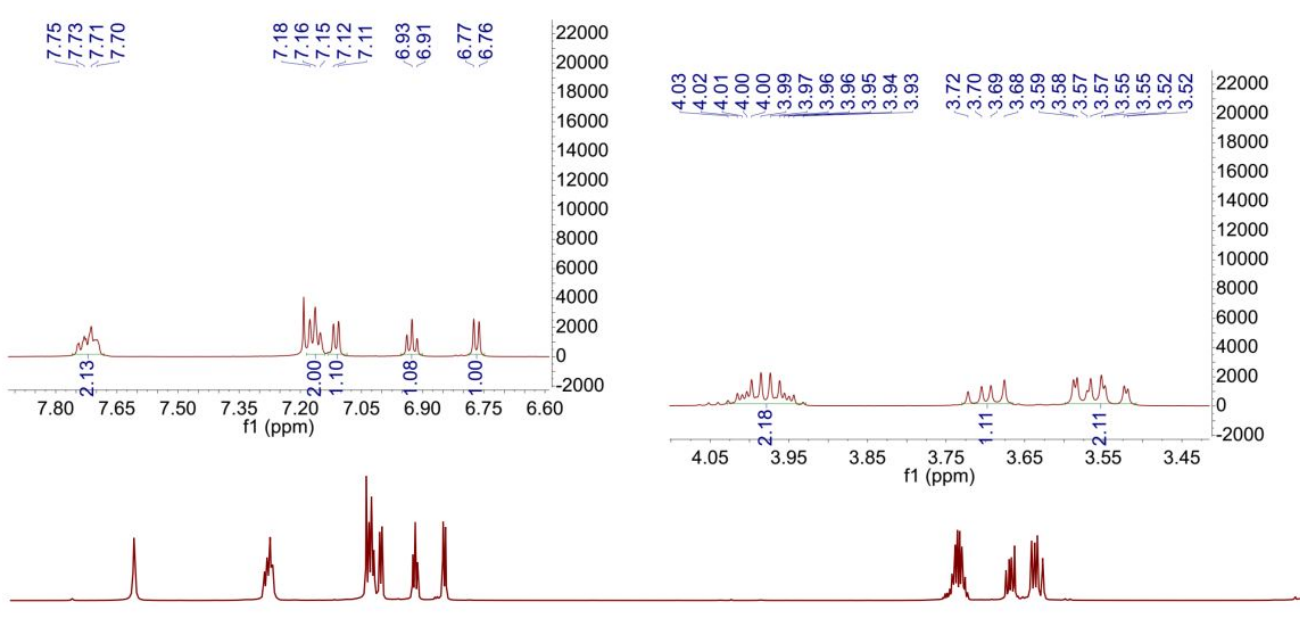

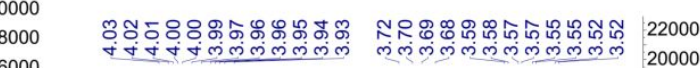
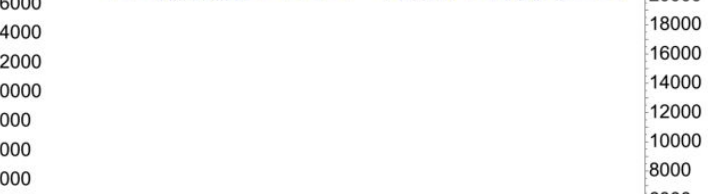

6000

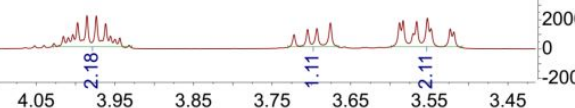

$$
000
$$

\begin{tabular}{|c|c|c|c|c|c|c|c|c|c|c|c|c|c|}
\hline & $\begin{array}{l}\text { T' } \\
\stackrel{8}{\circ}\end{array}$ & & & 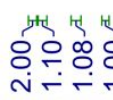 & & & & & & 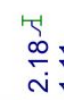 & & & \\
\hline 10 & 8.5 & 8.0 & 7.5 & 7.0 & 6.5 & 6.0 & 5.5 & 5.0 & 4.5 & 4.0 & 3.5 & 3.0 & 2.5 \\
\hline
\end{tabular}

f1 (ppm) 

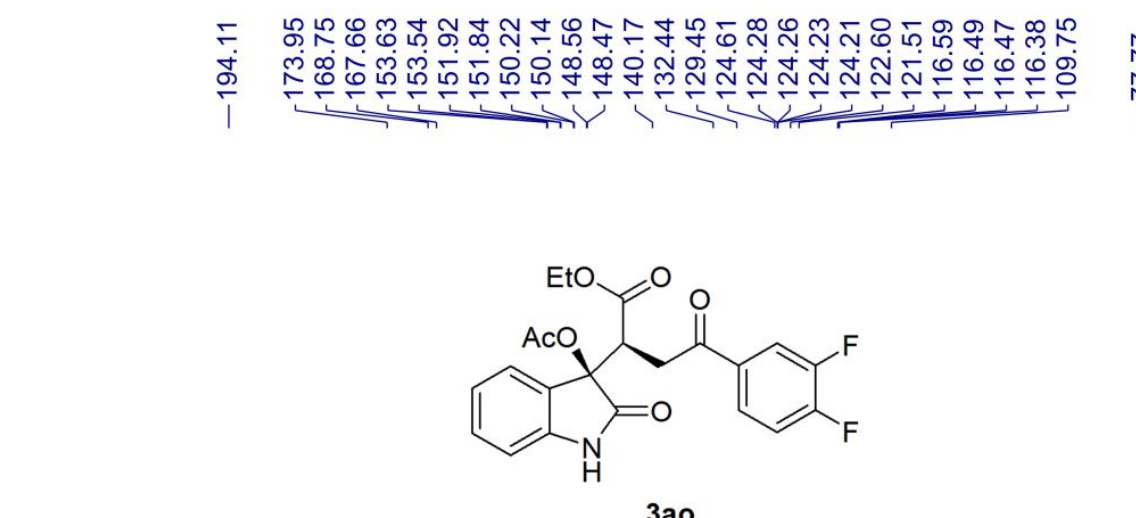

$-120000$

${ }^{13} \mathrm{C}\left\{{ }^{1} \mathrm{H}\right\}\left(150 \mathrm{MHz}, \mathrm{CDCl}_{3}\right)$

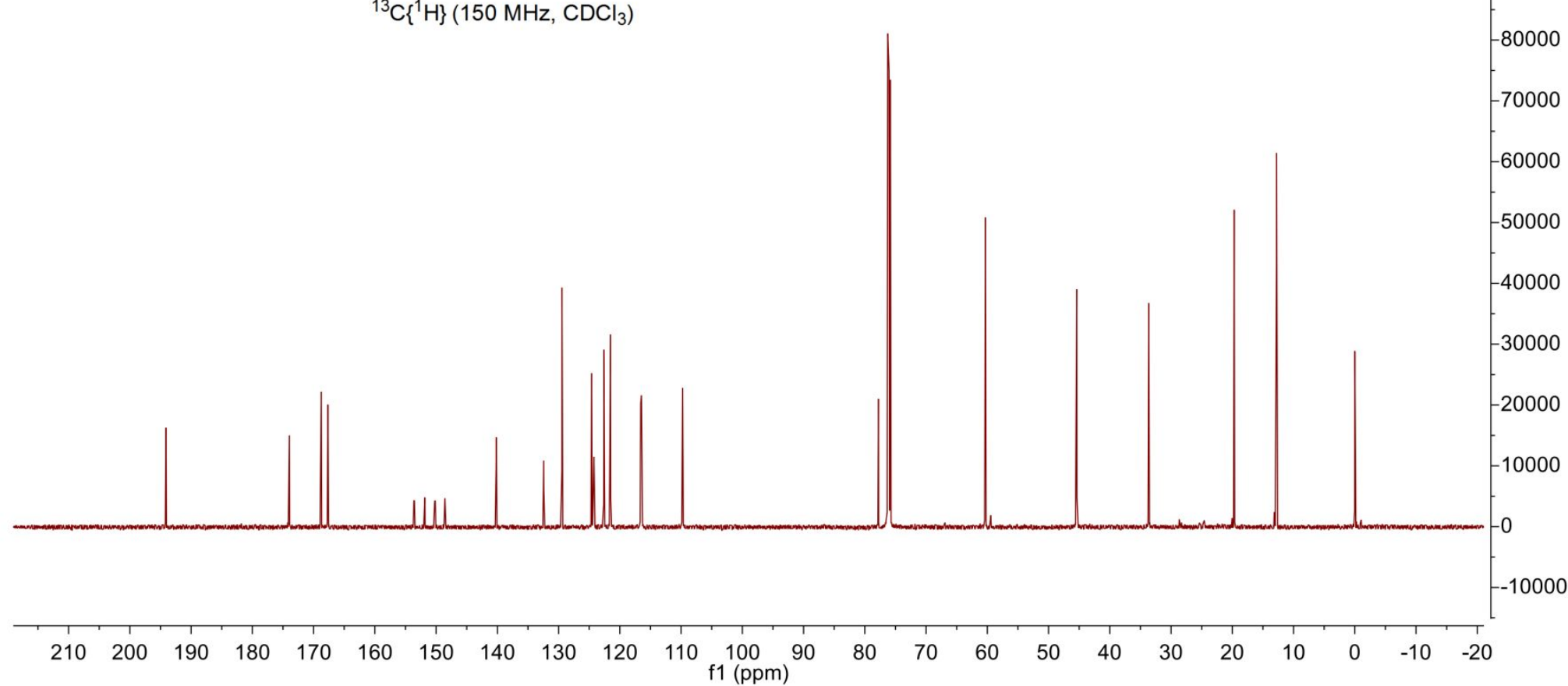

11000

100000

90000

80000

60000

50000

40000

30000

10000 


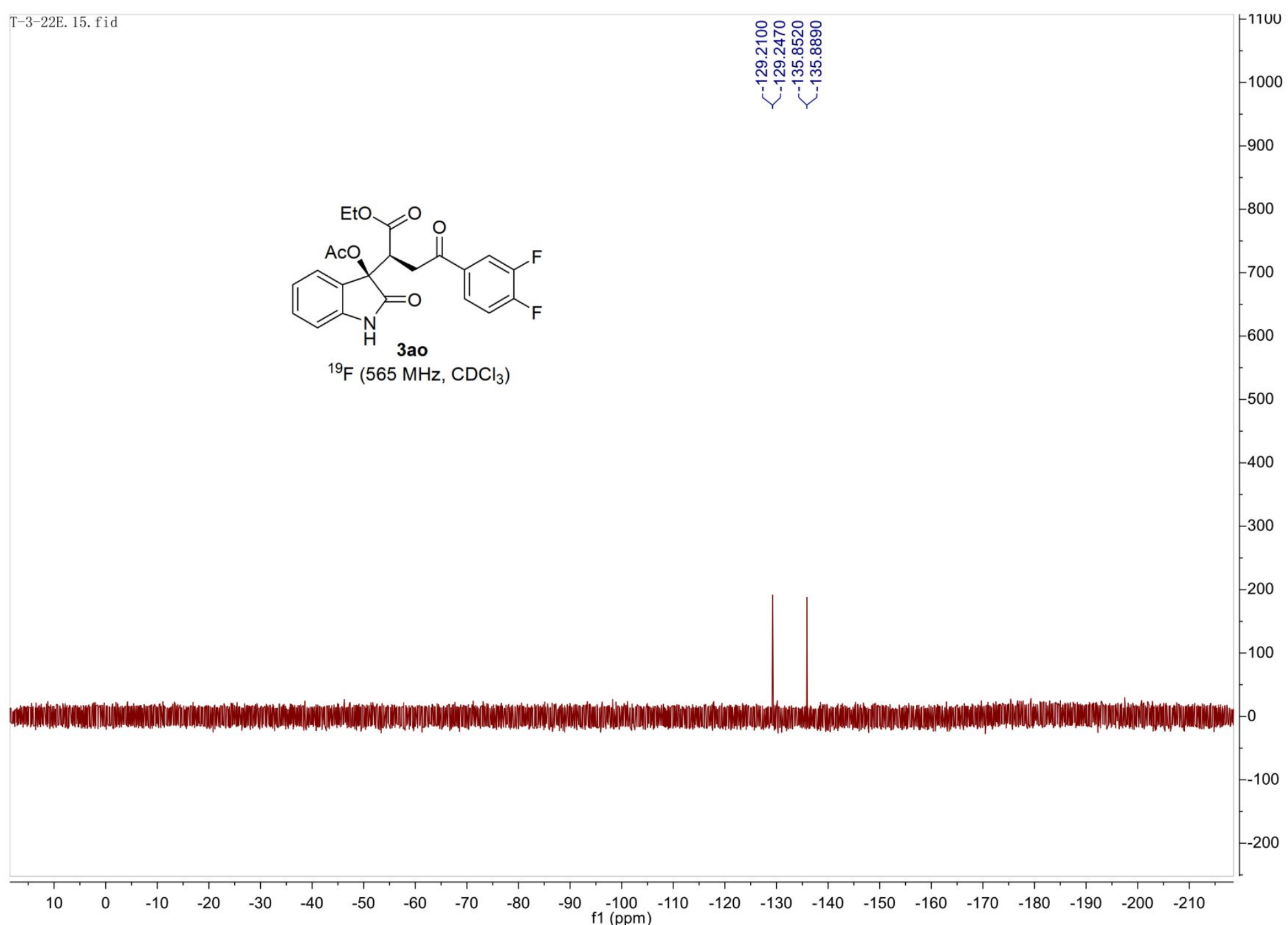




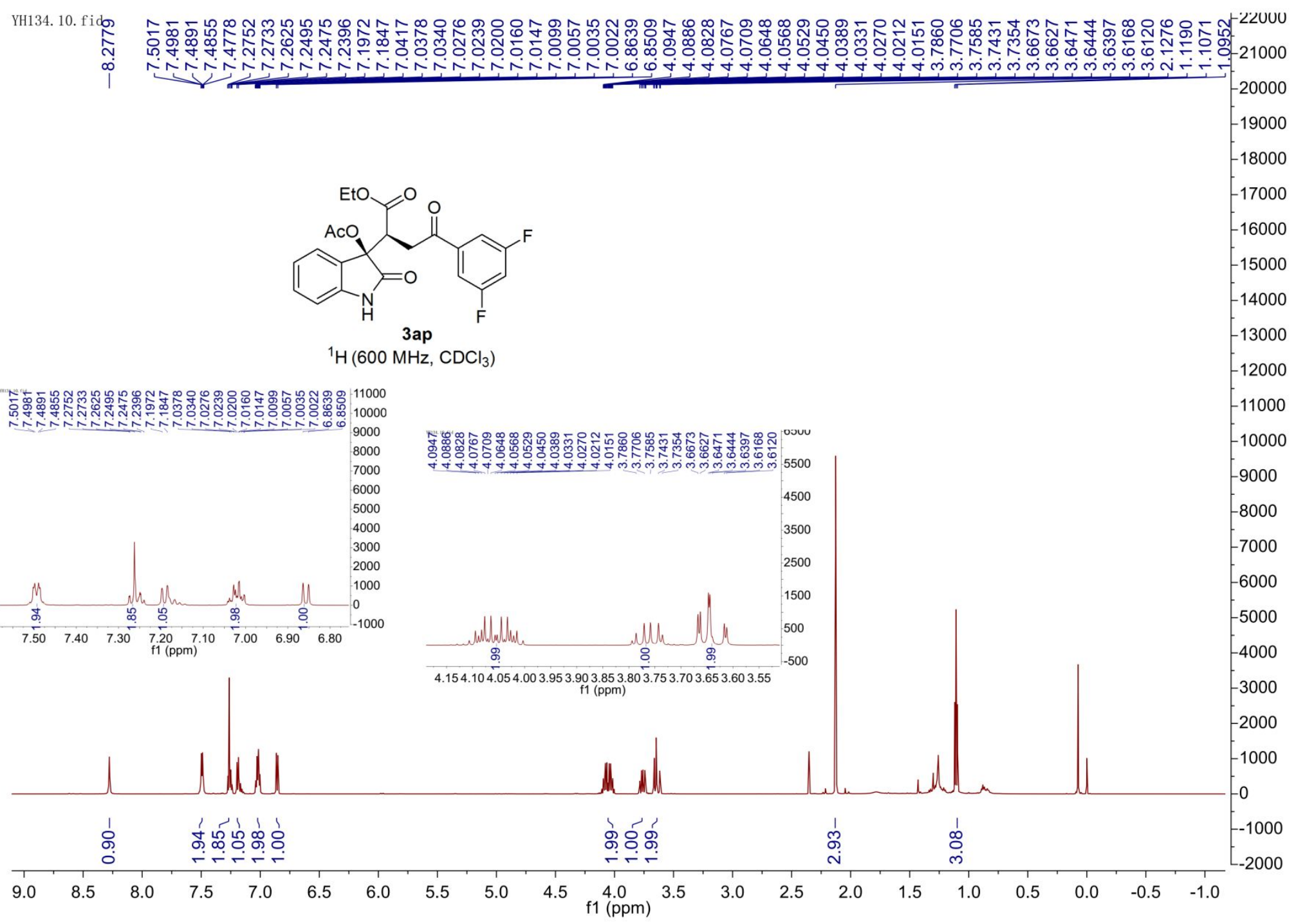




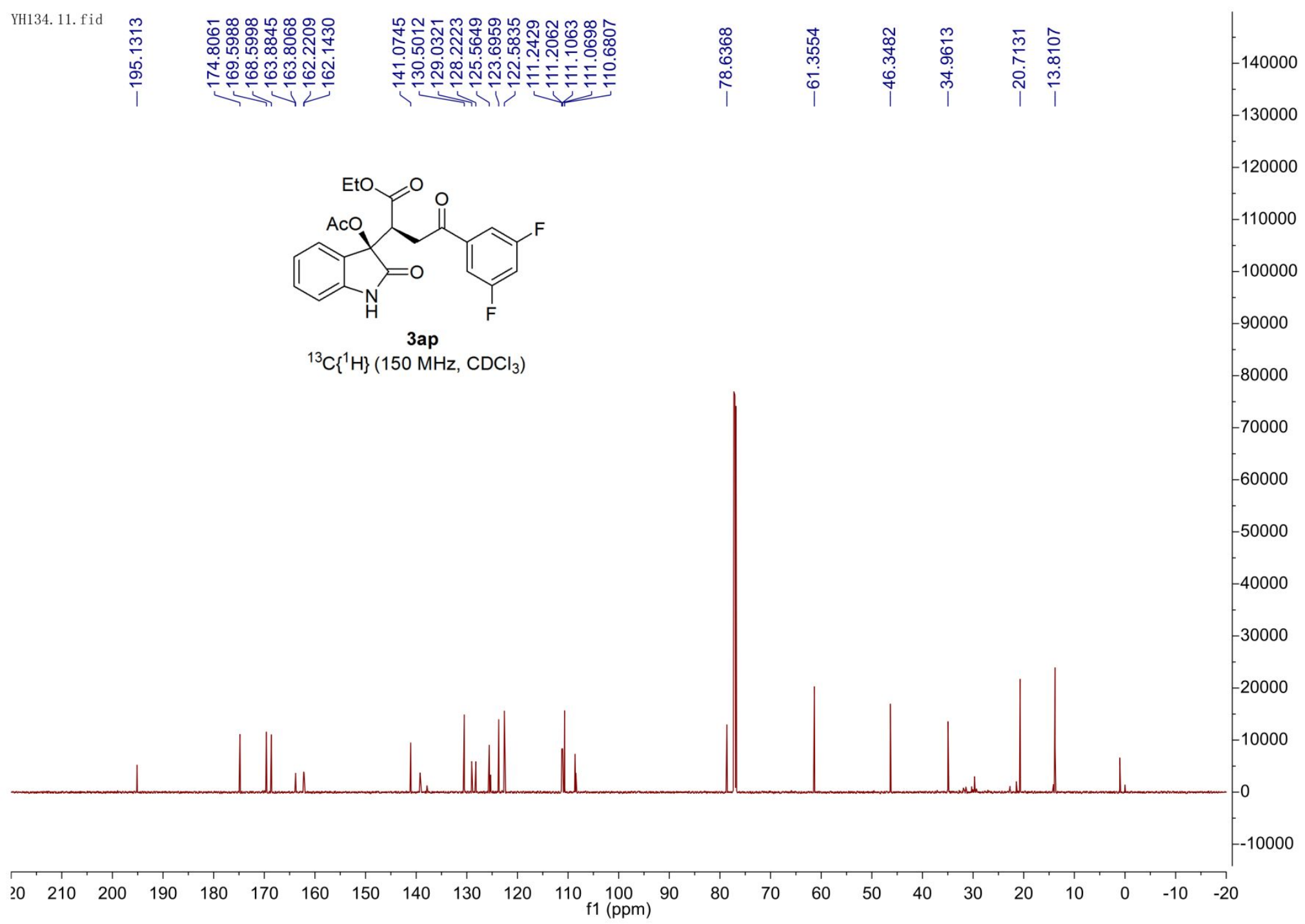




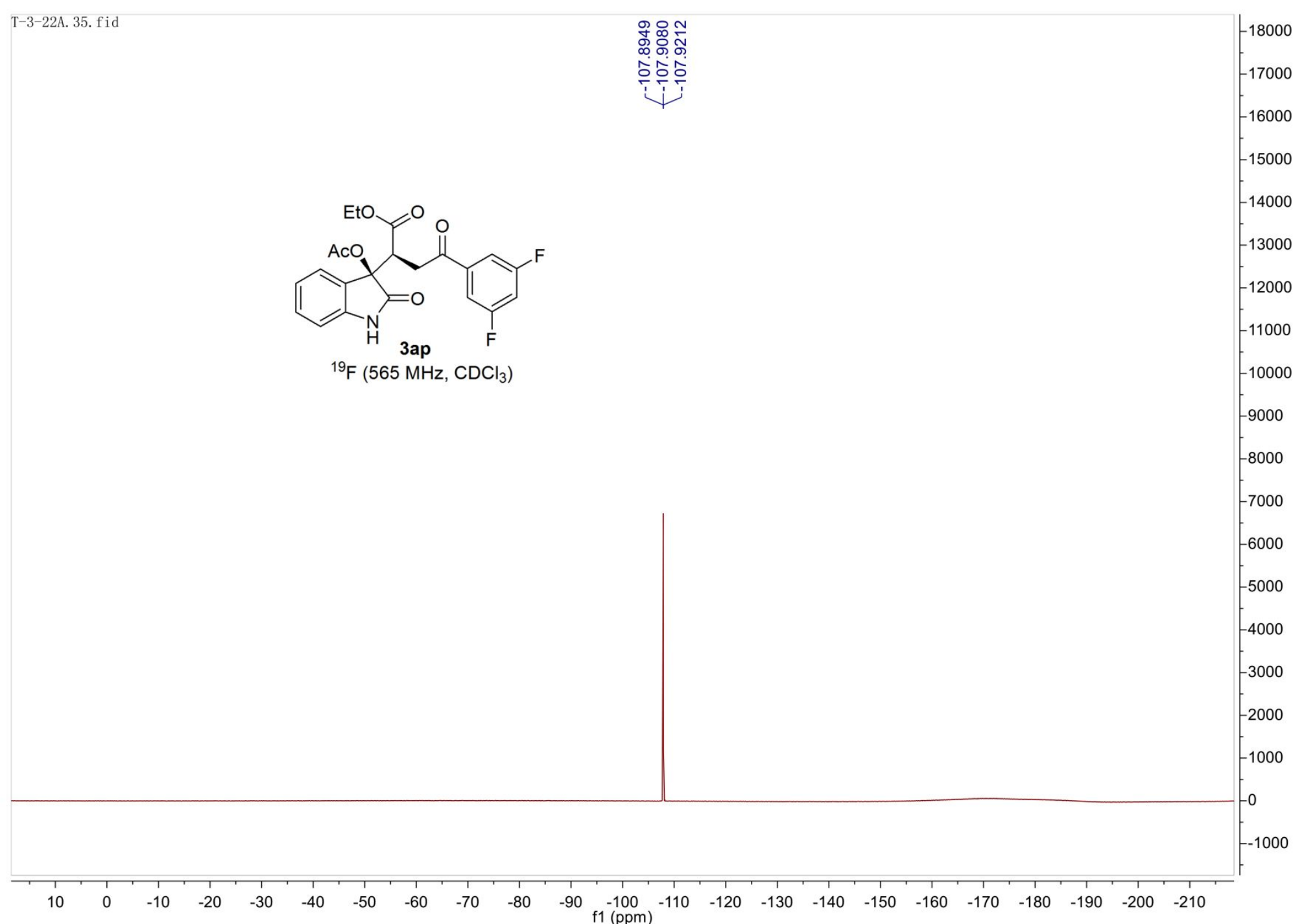




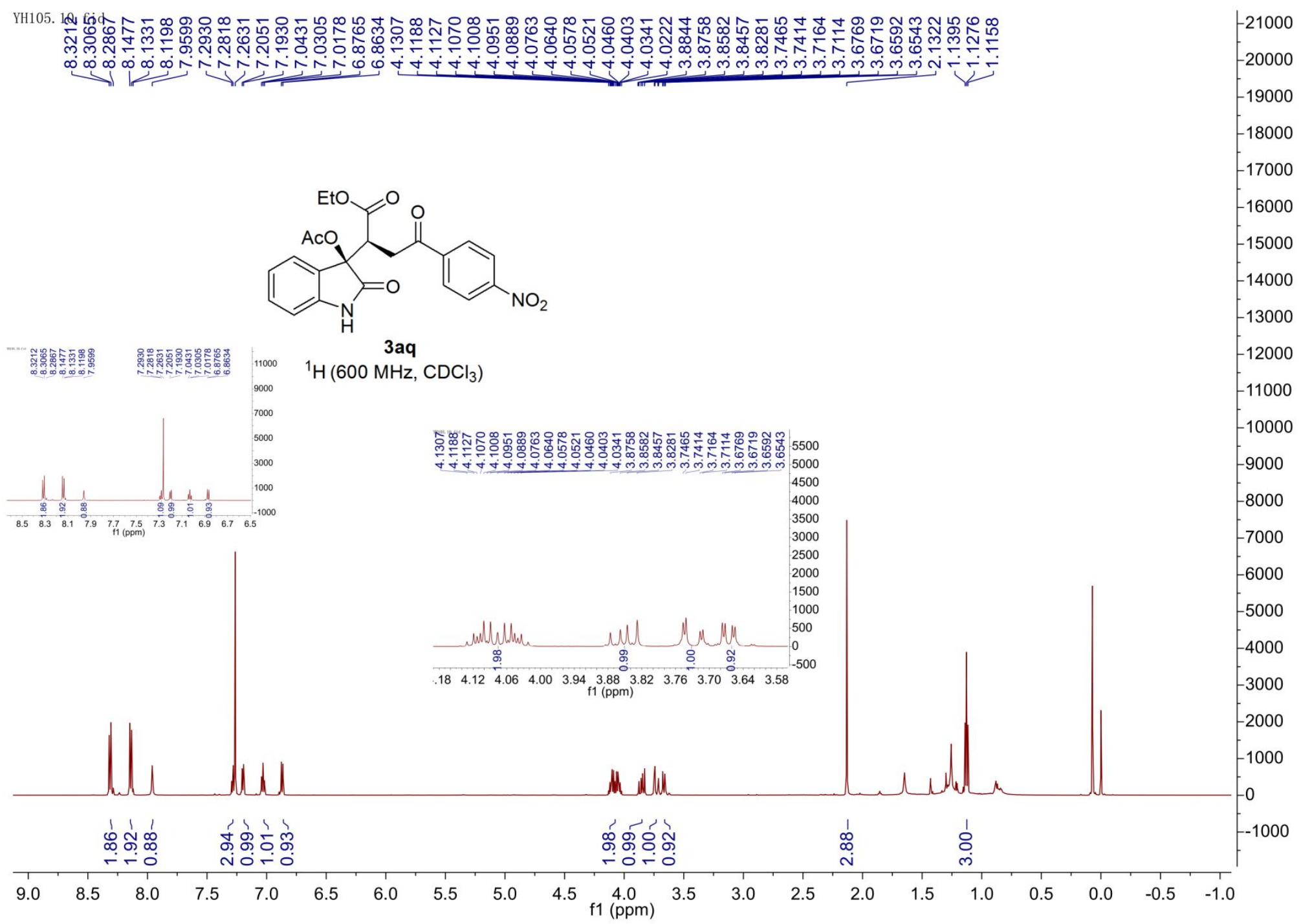




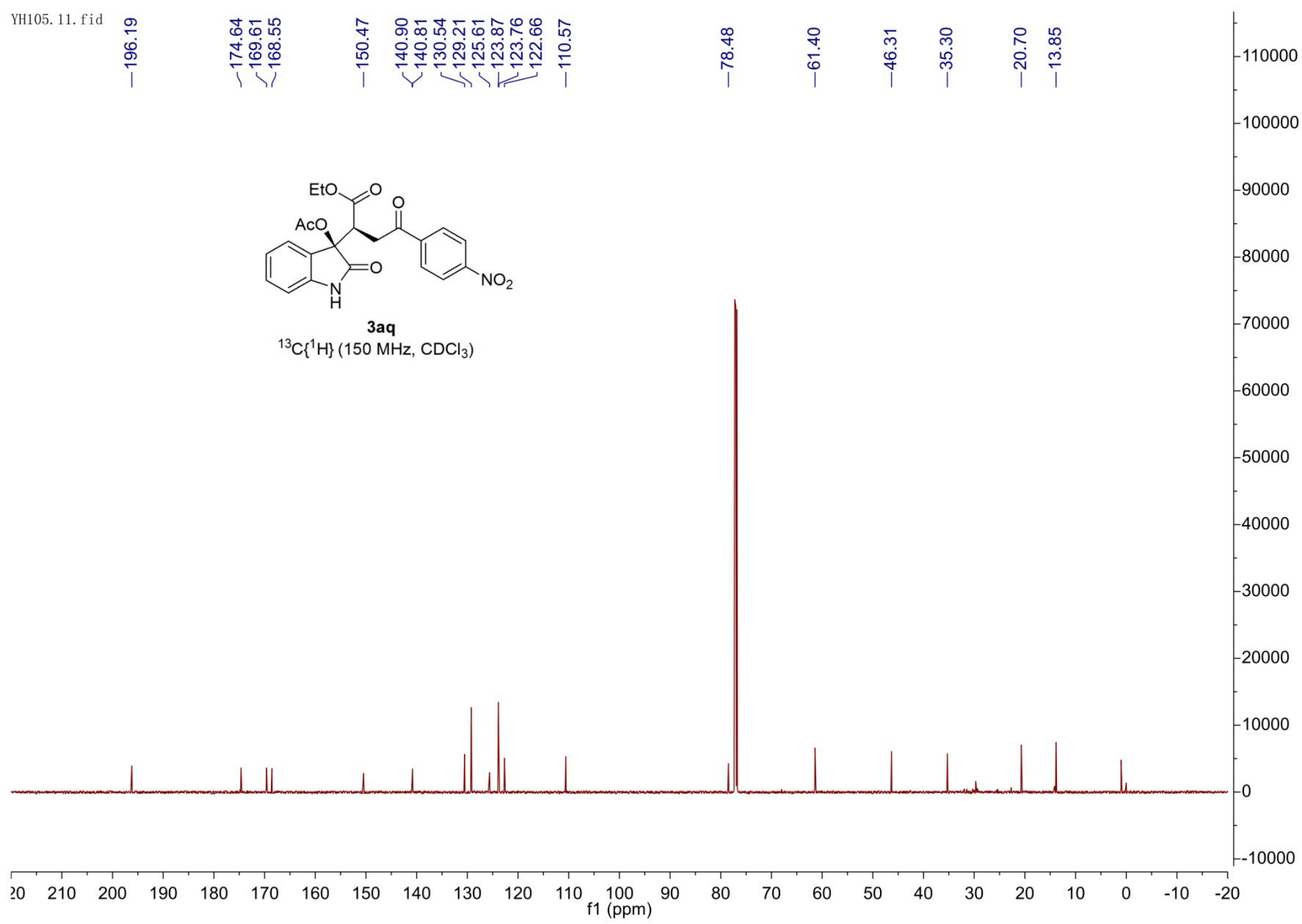




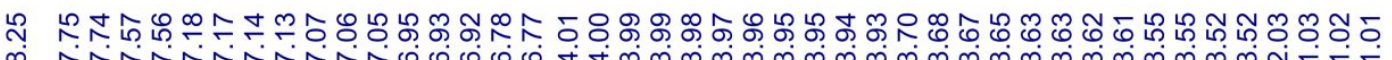

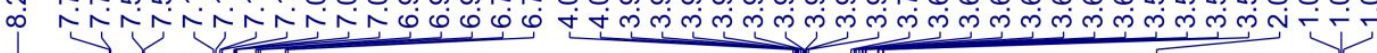

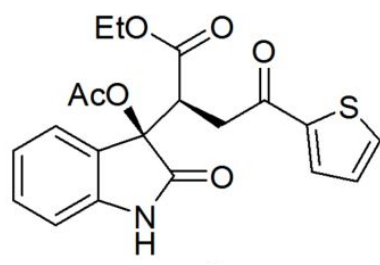

$-20000$

$-18000$

$-16000$

$3 a r$

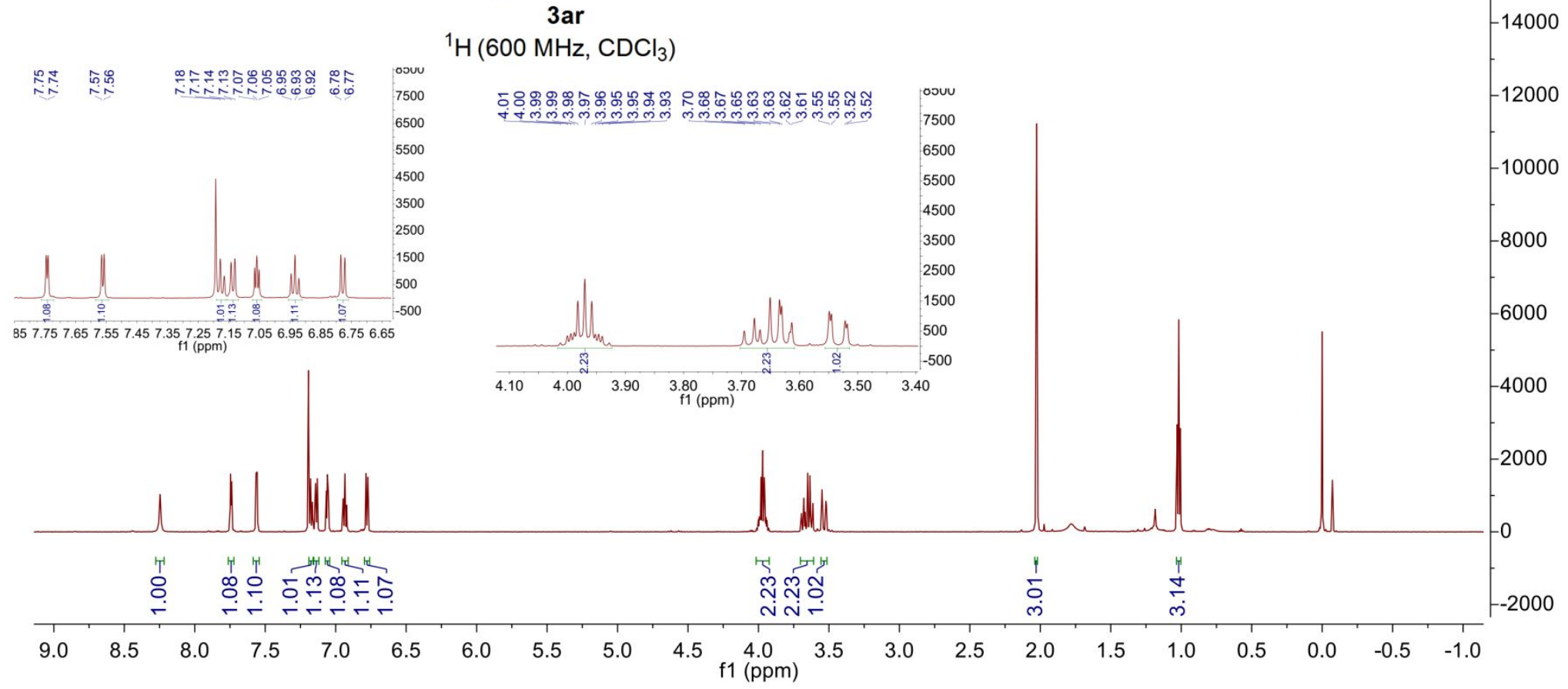




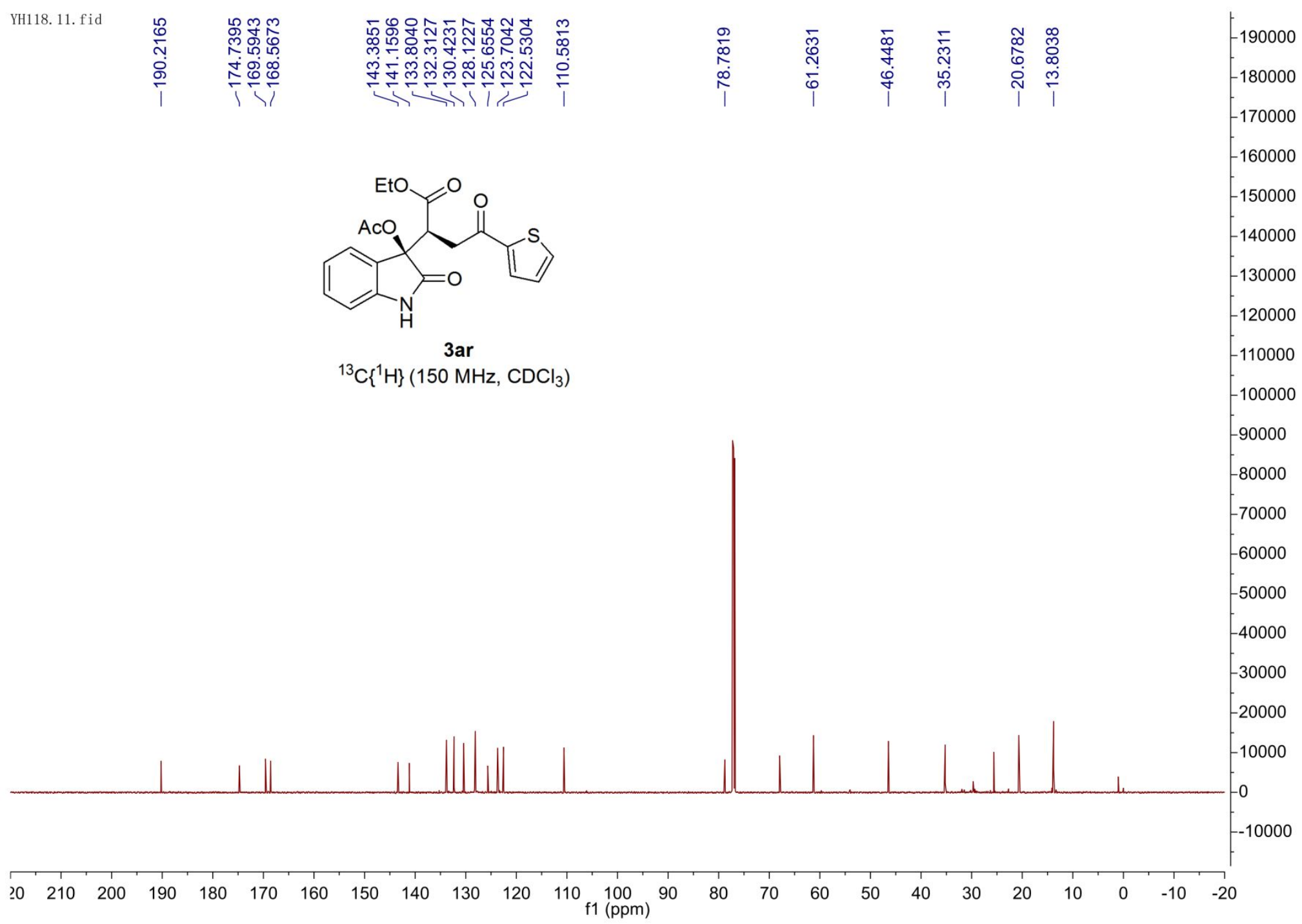




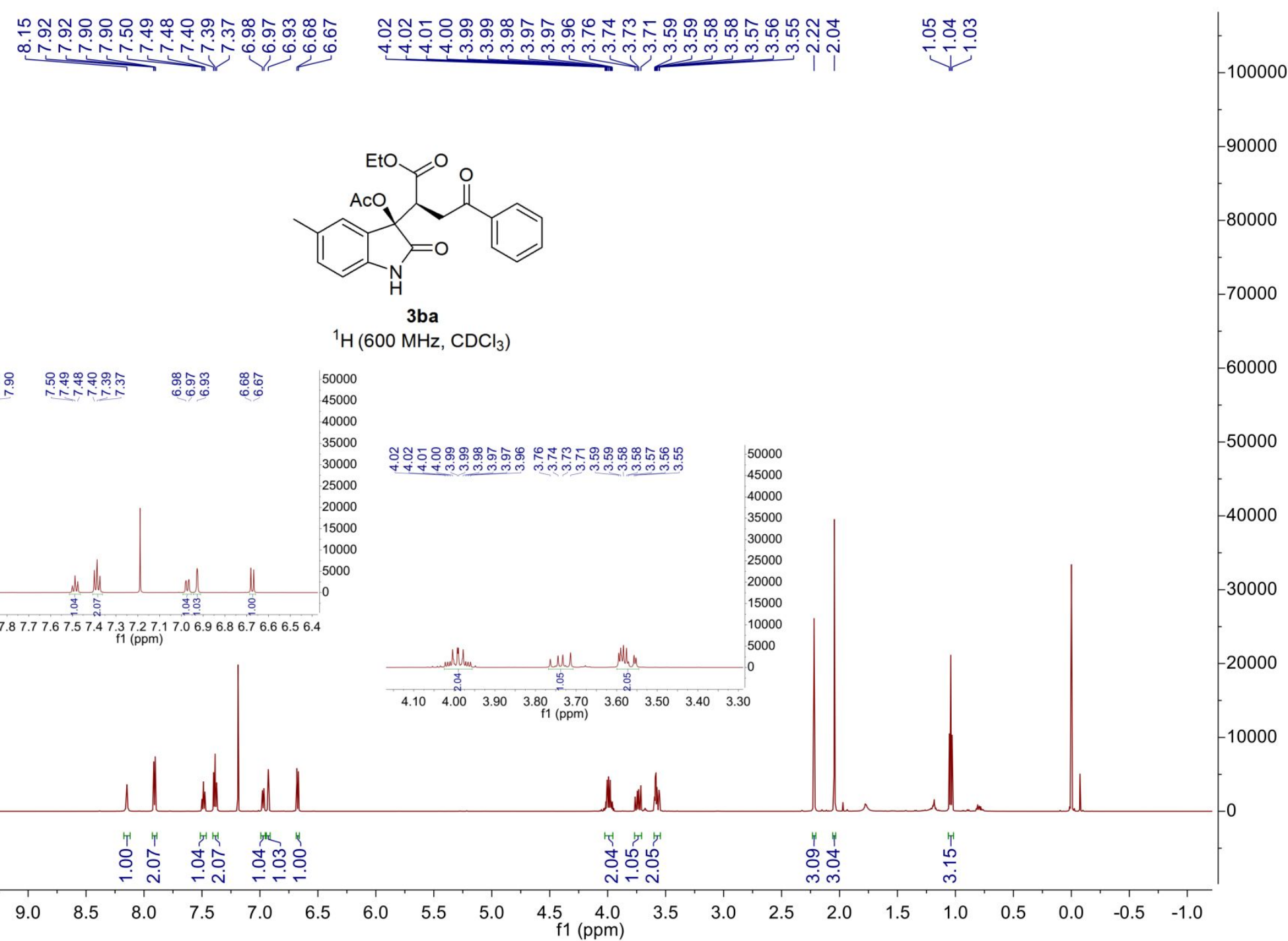




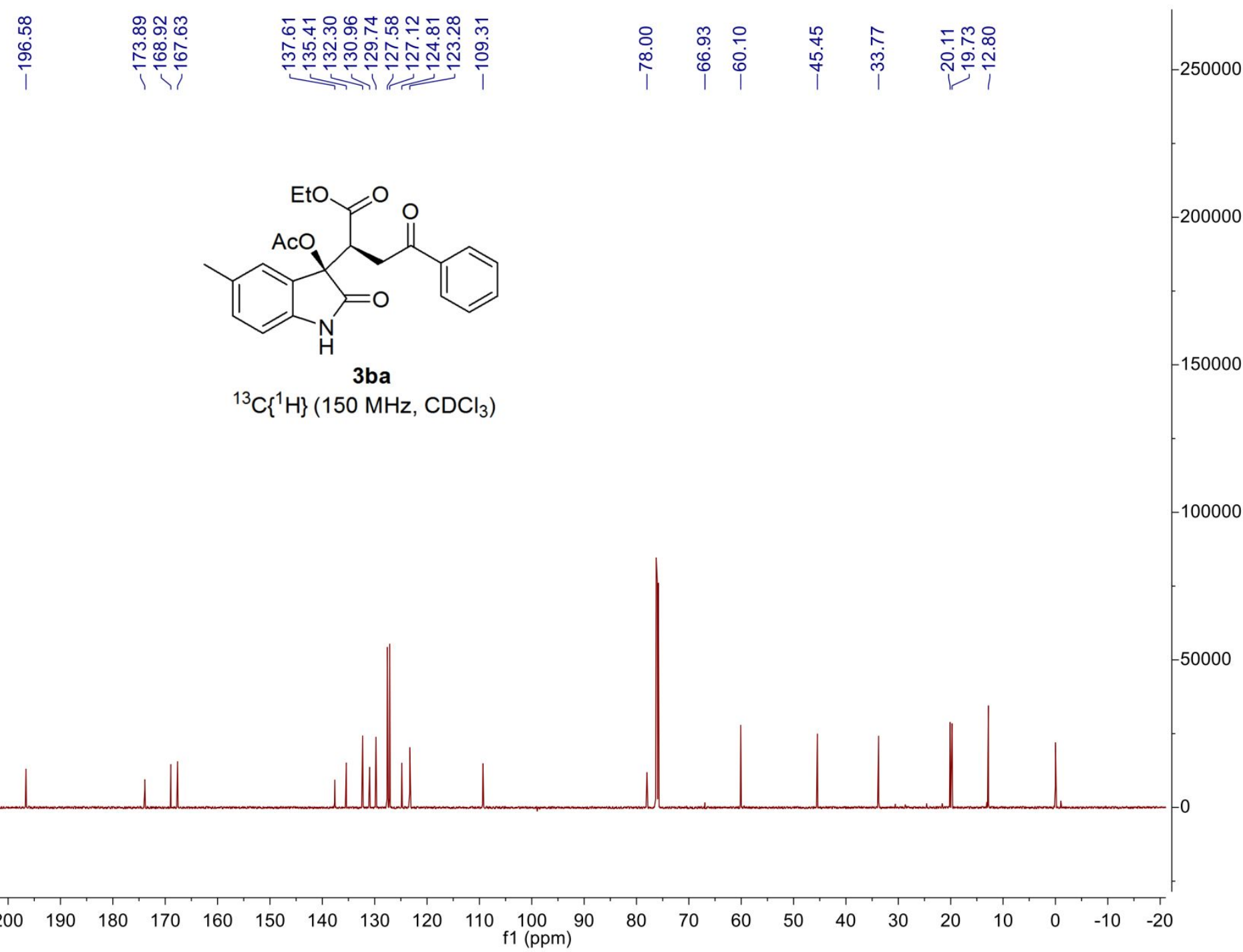




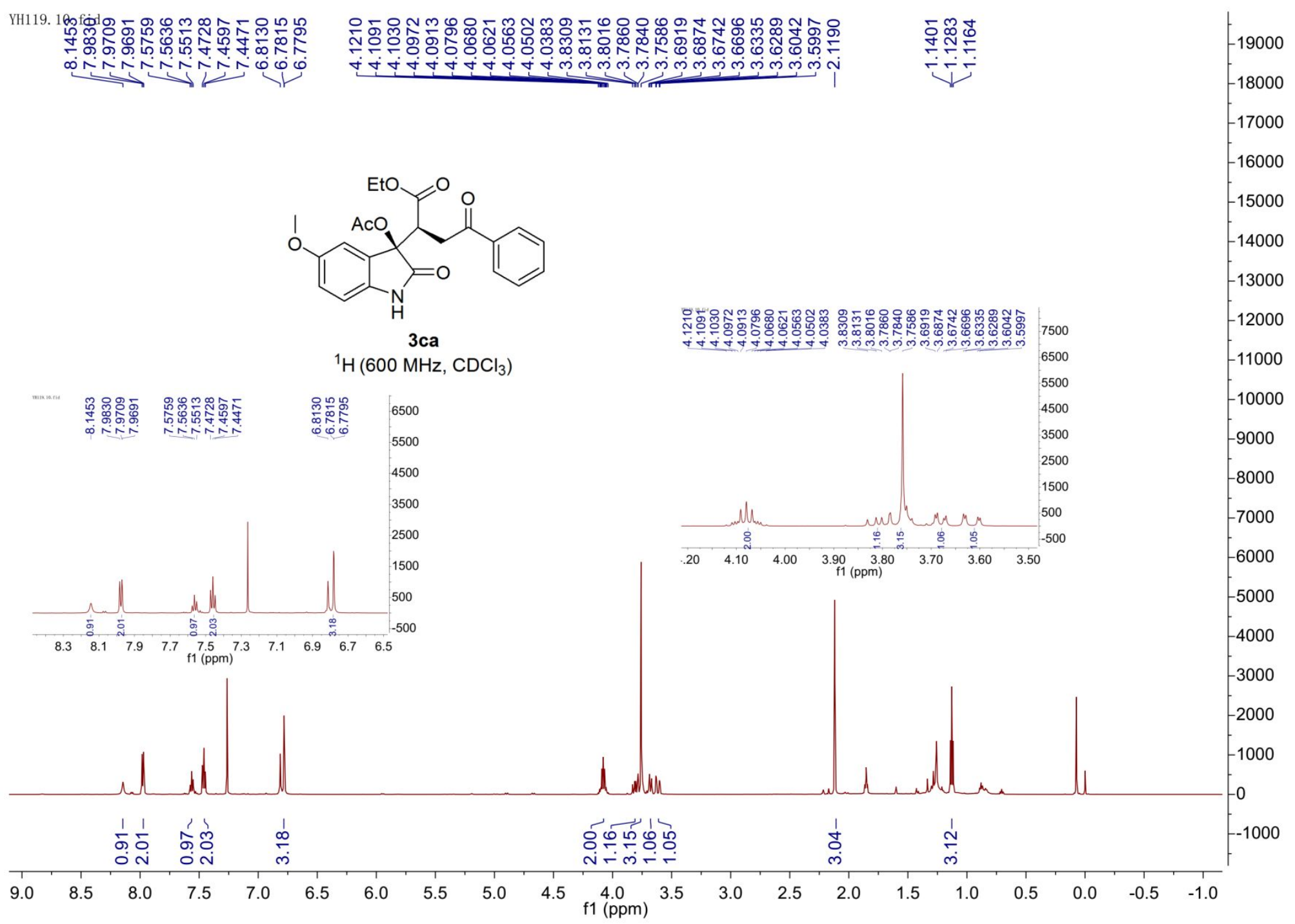




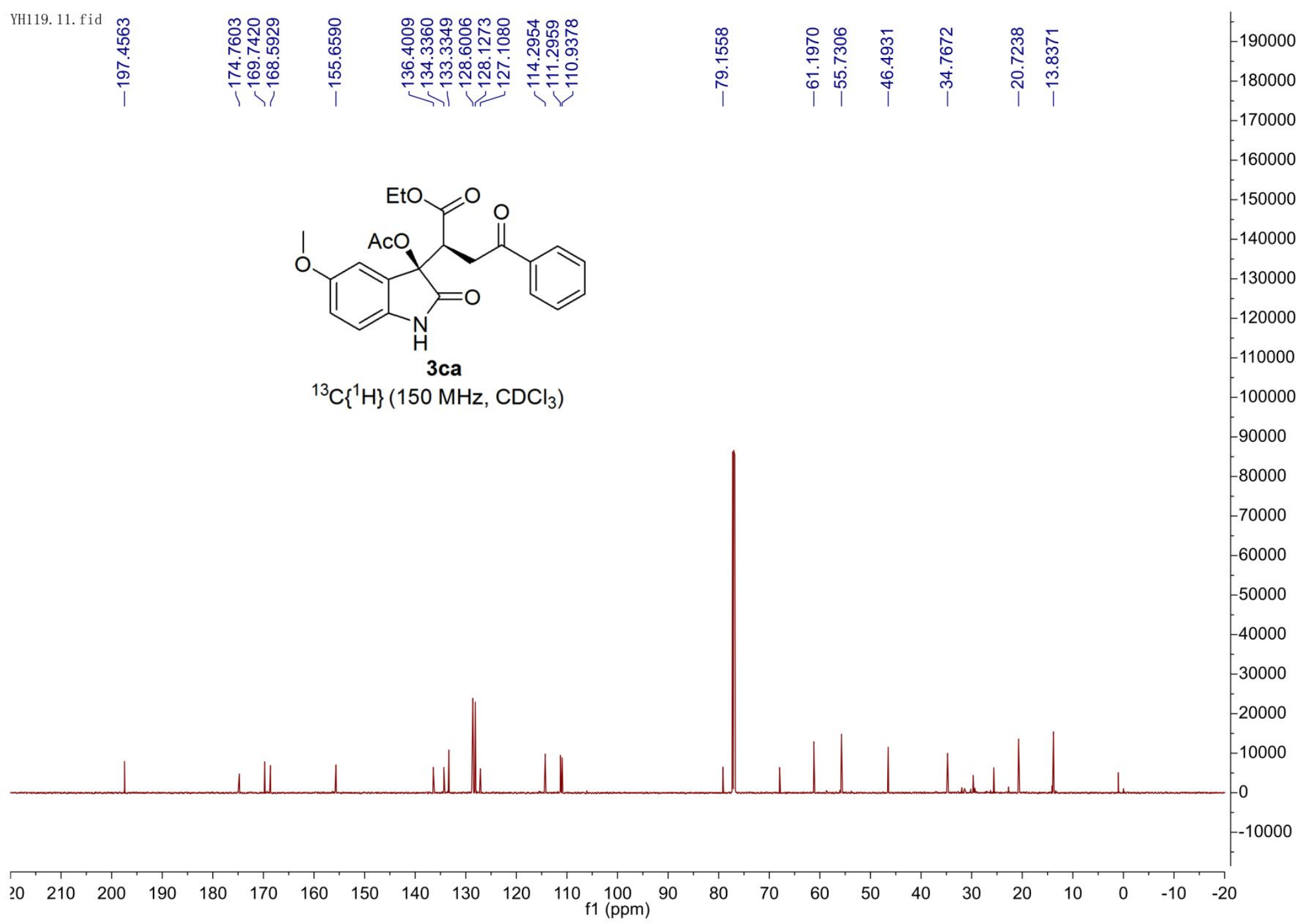




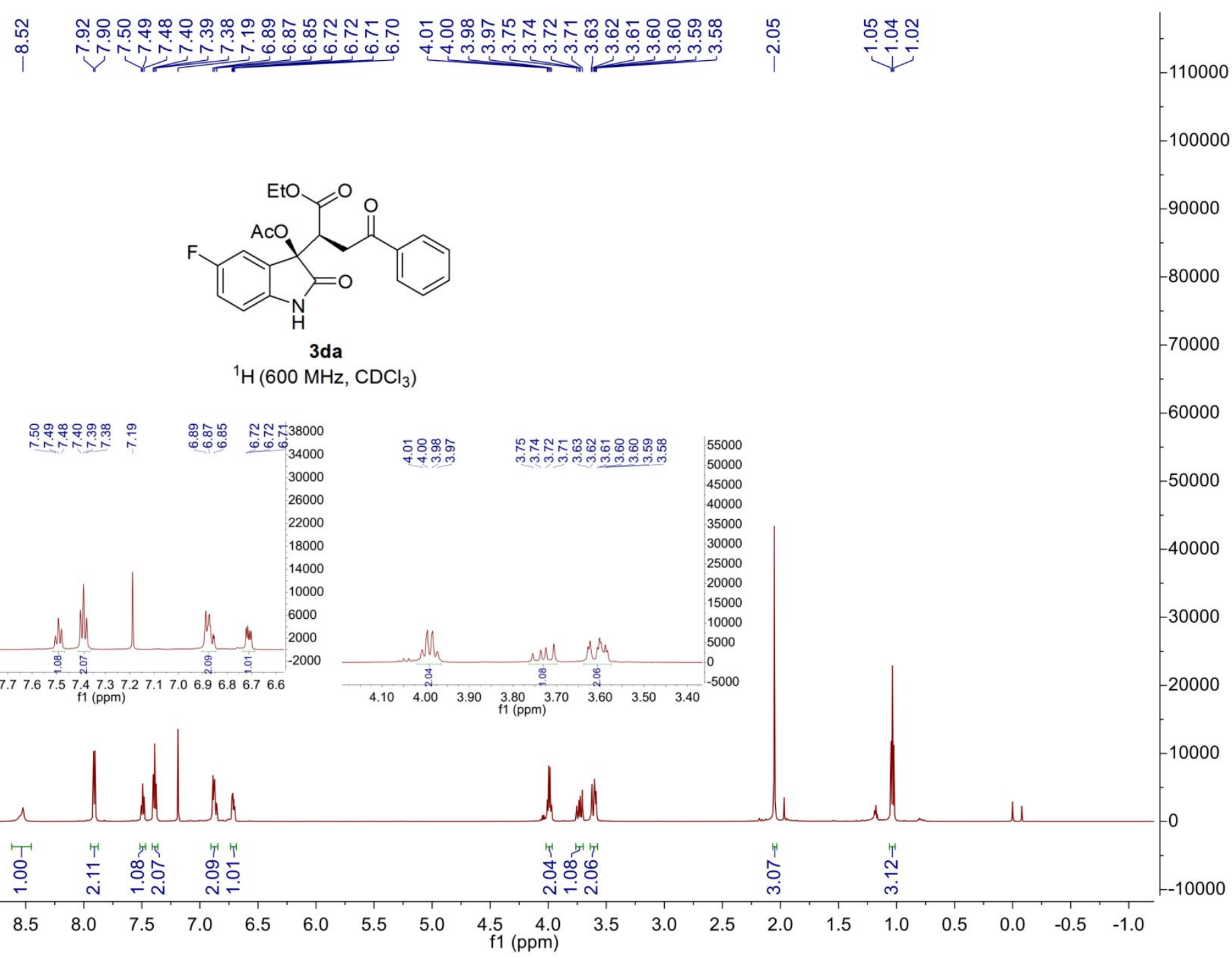




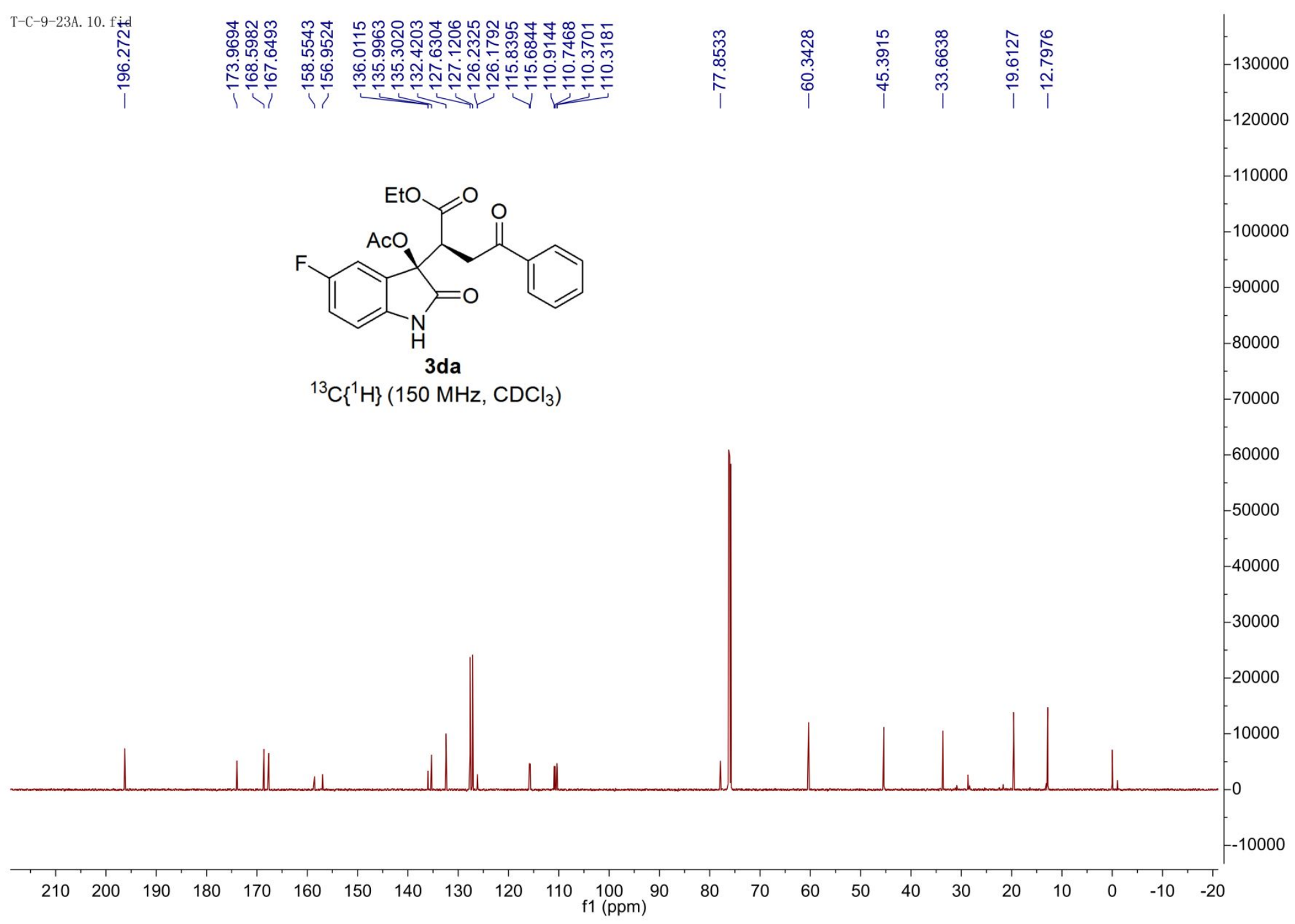




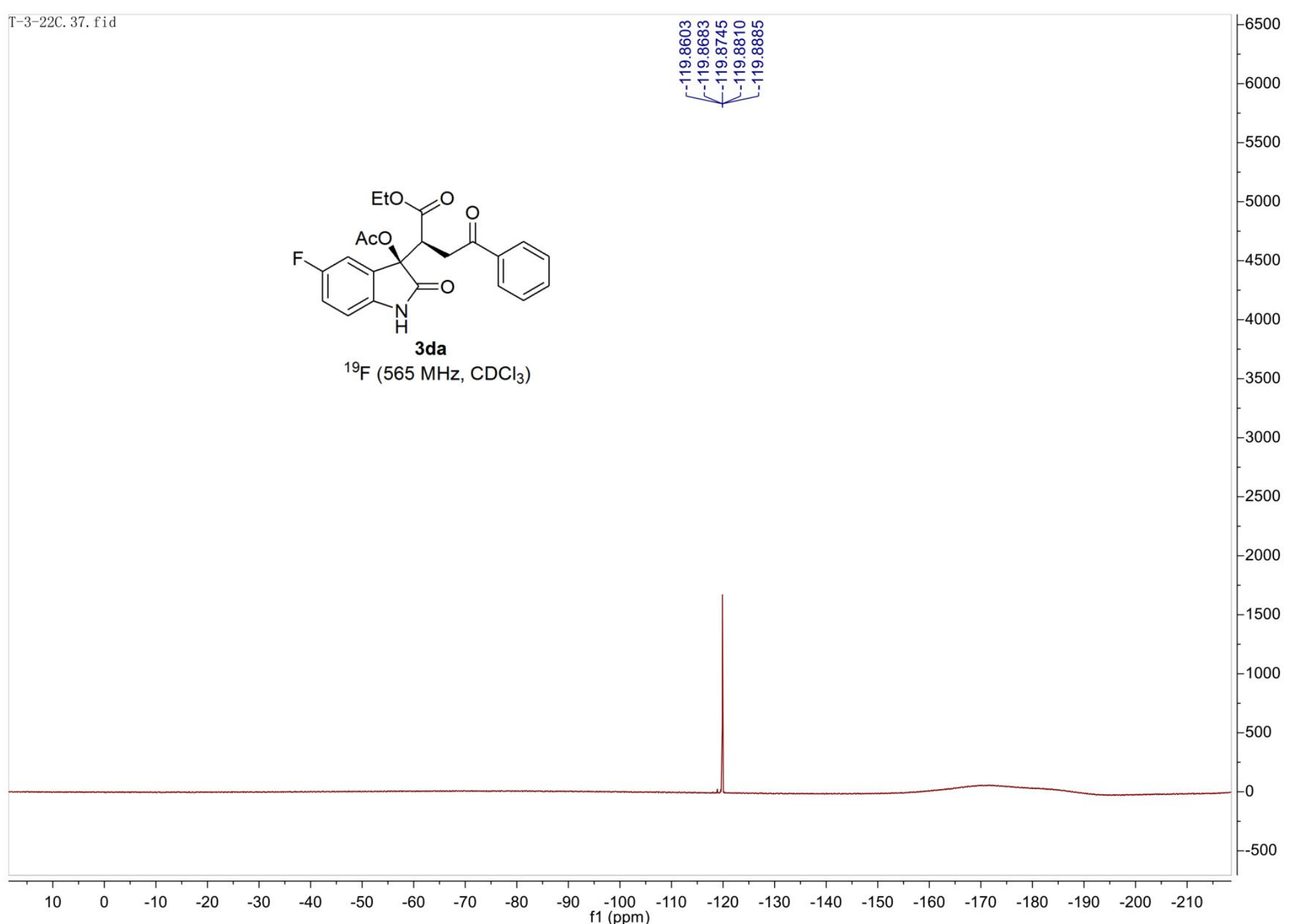




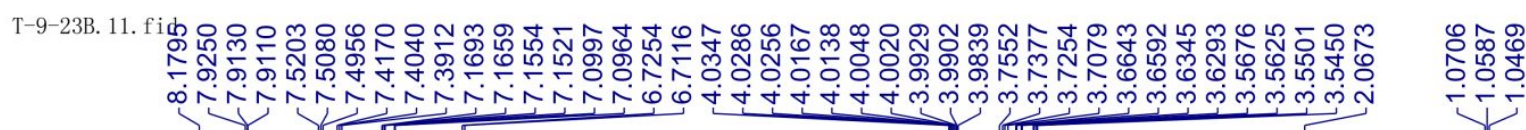

$-24000$

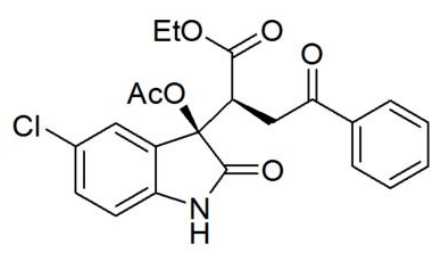

$-22000$

0000

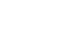

18000

3 ea

$\mathrm{H}\left(600 \mathrm{MHz}, \mathrm{CDCl}_{3}\right)$

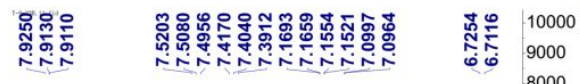

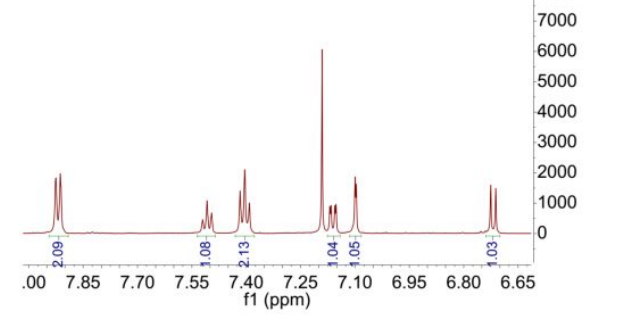

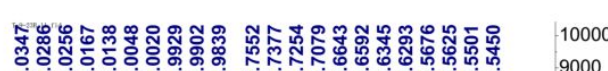

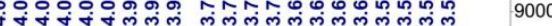
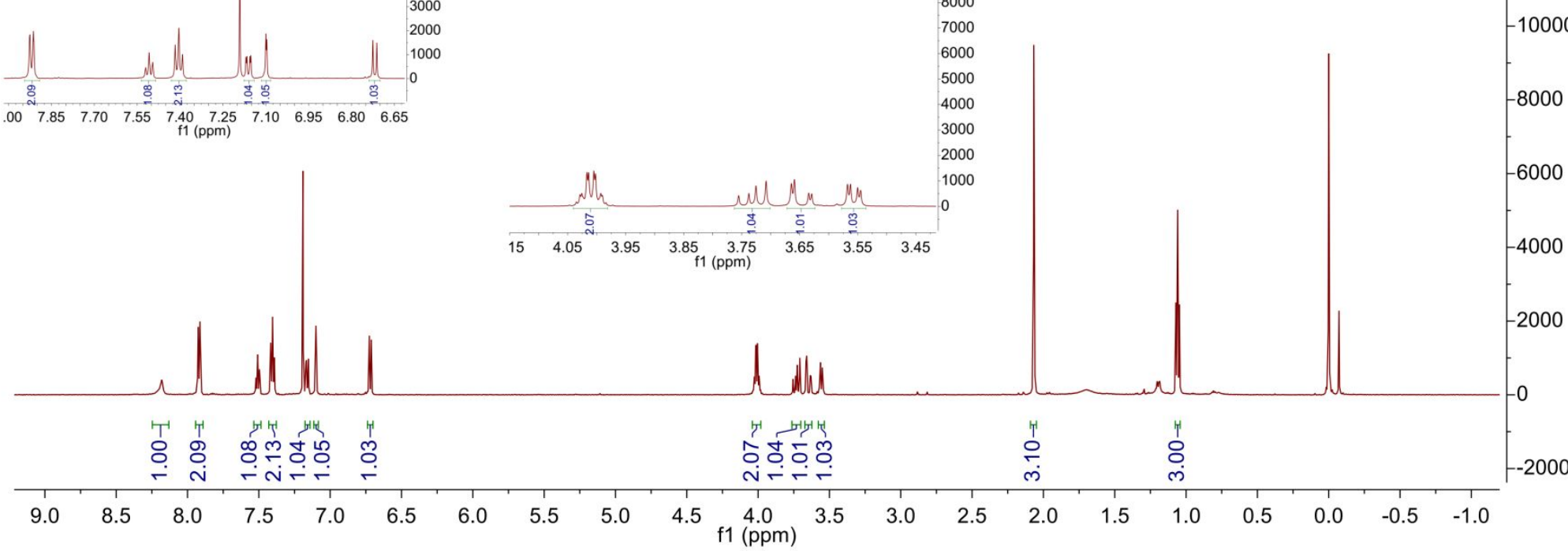


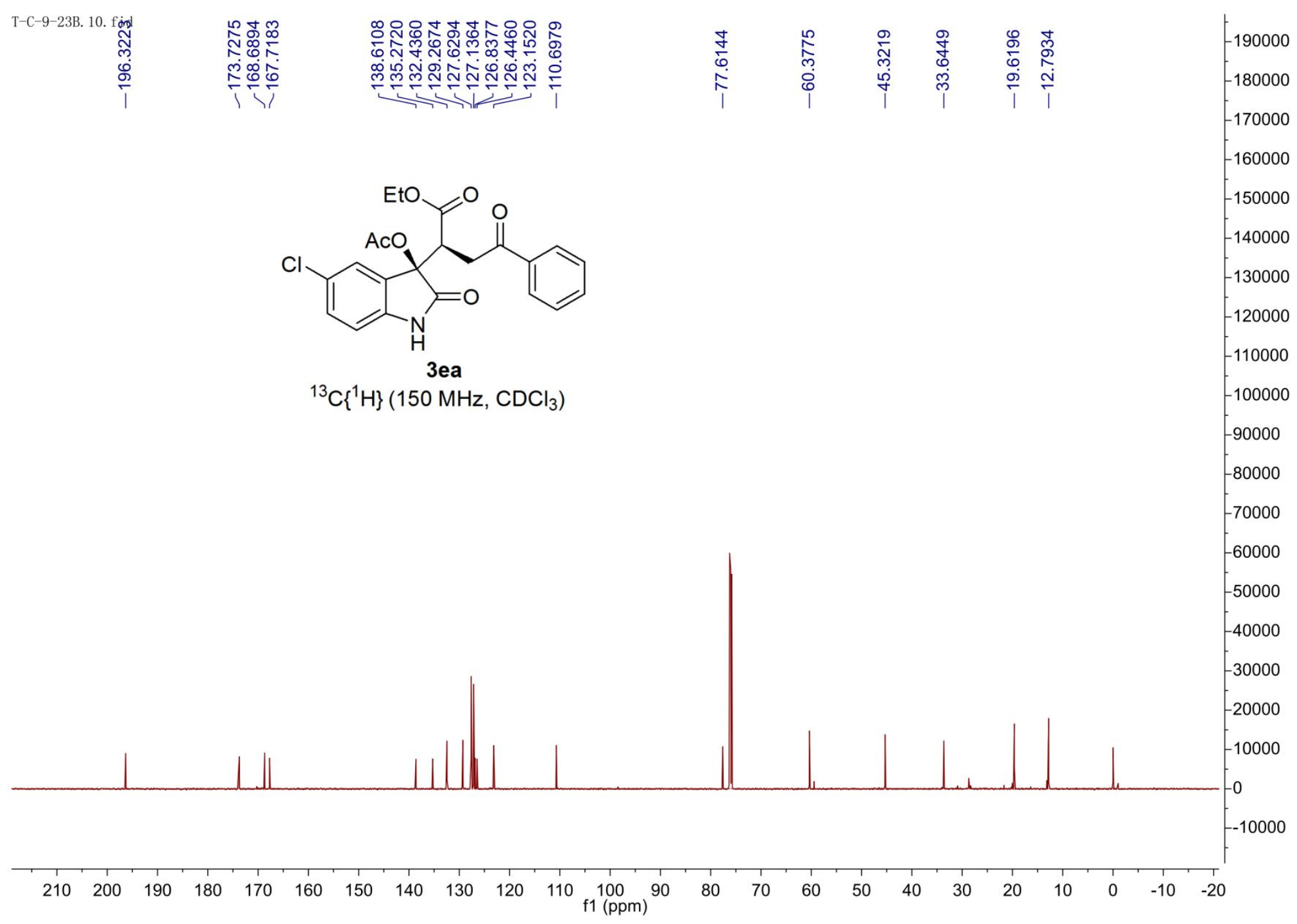




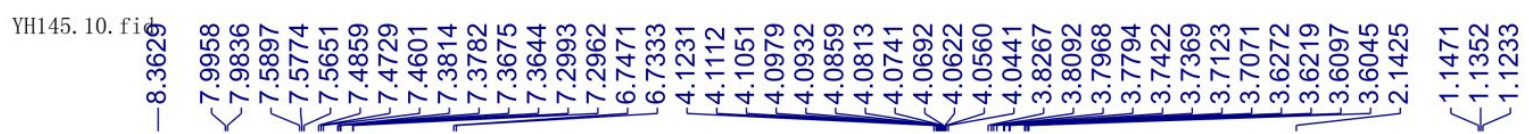

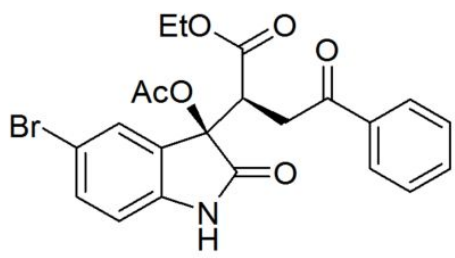

3 fa

22000

$-20000$

${ }^{1} \mathrm{H}\left(600 \mathrm{MHz}, \mathrm{CDCl}_{3}\right)$

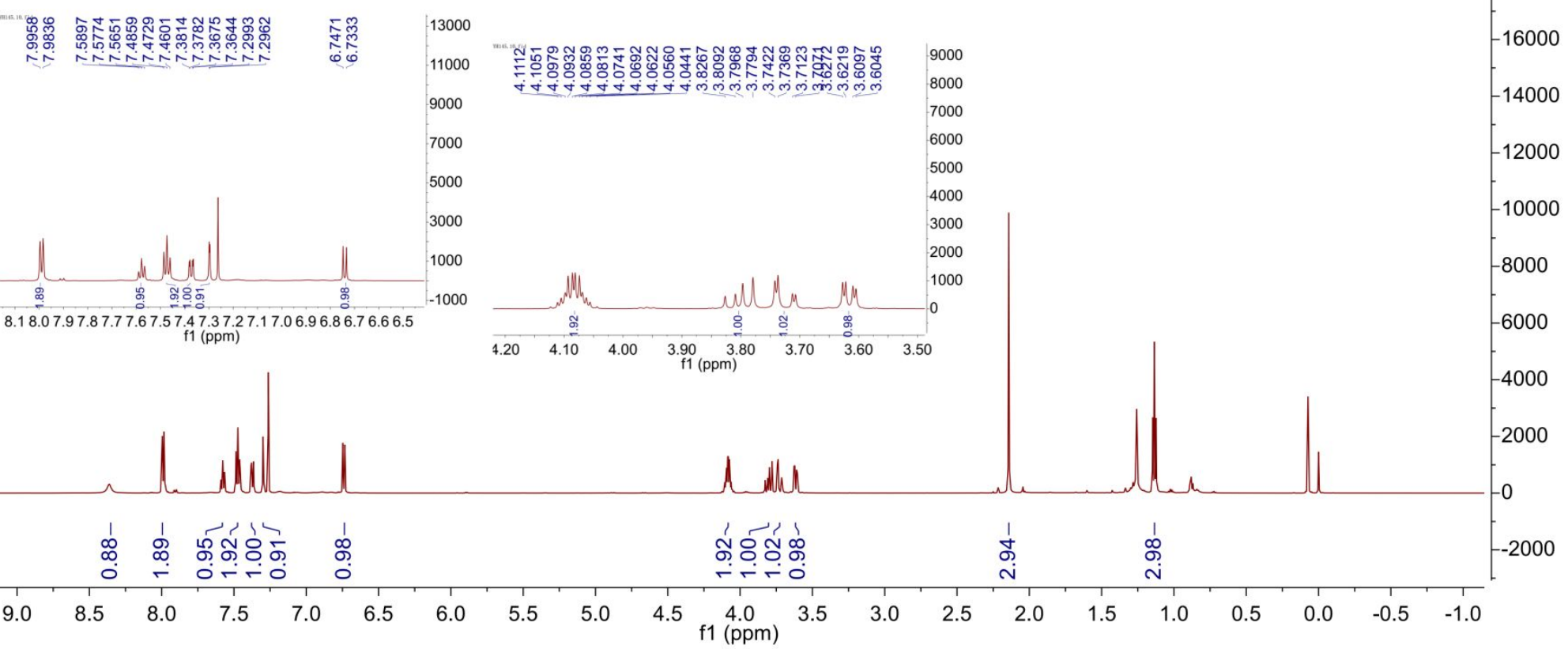




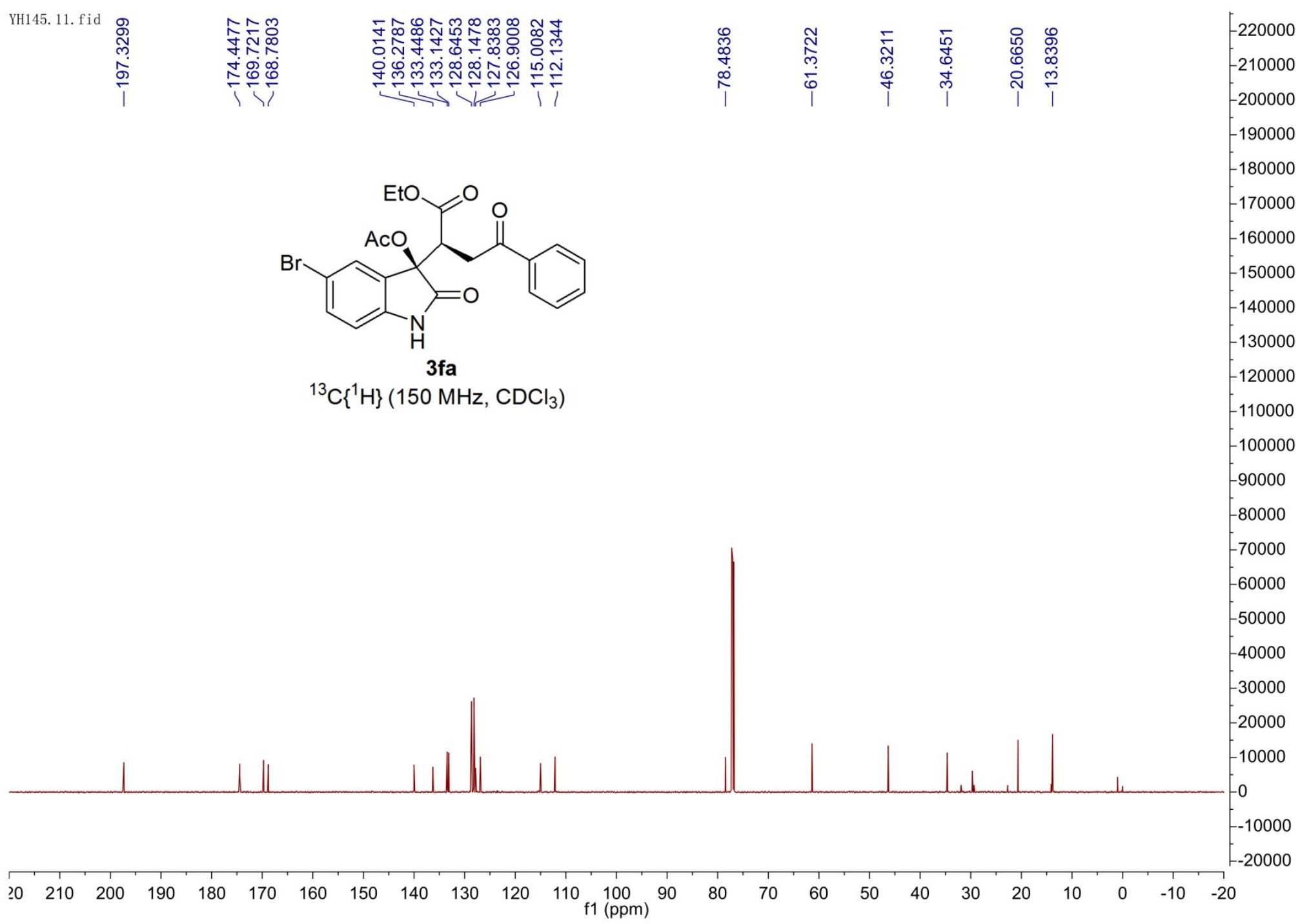




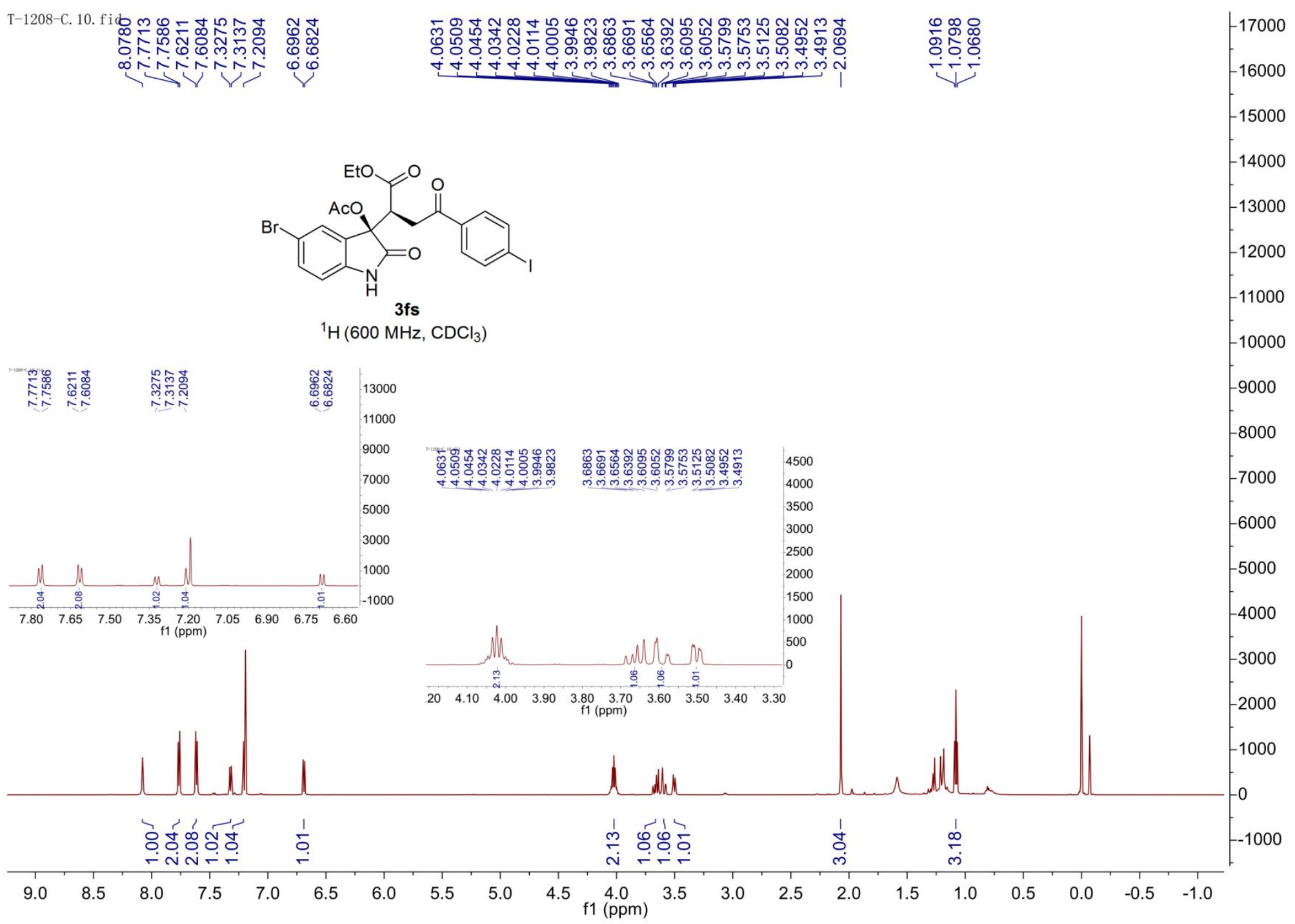




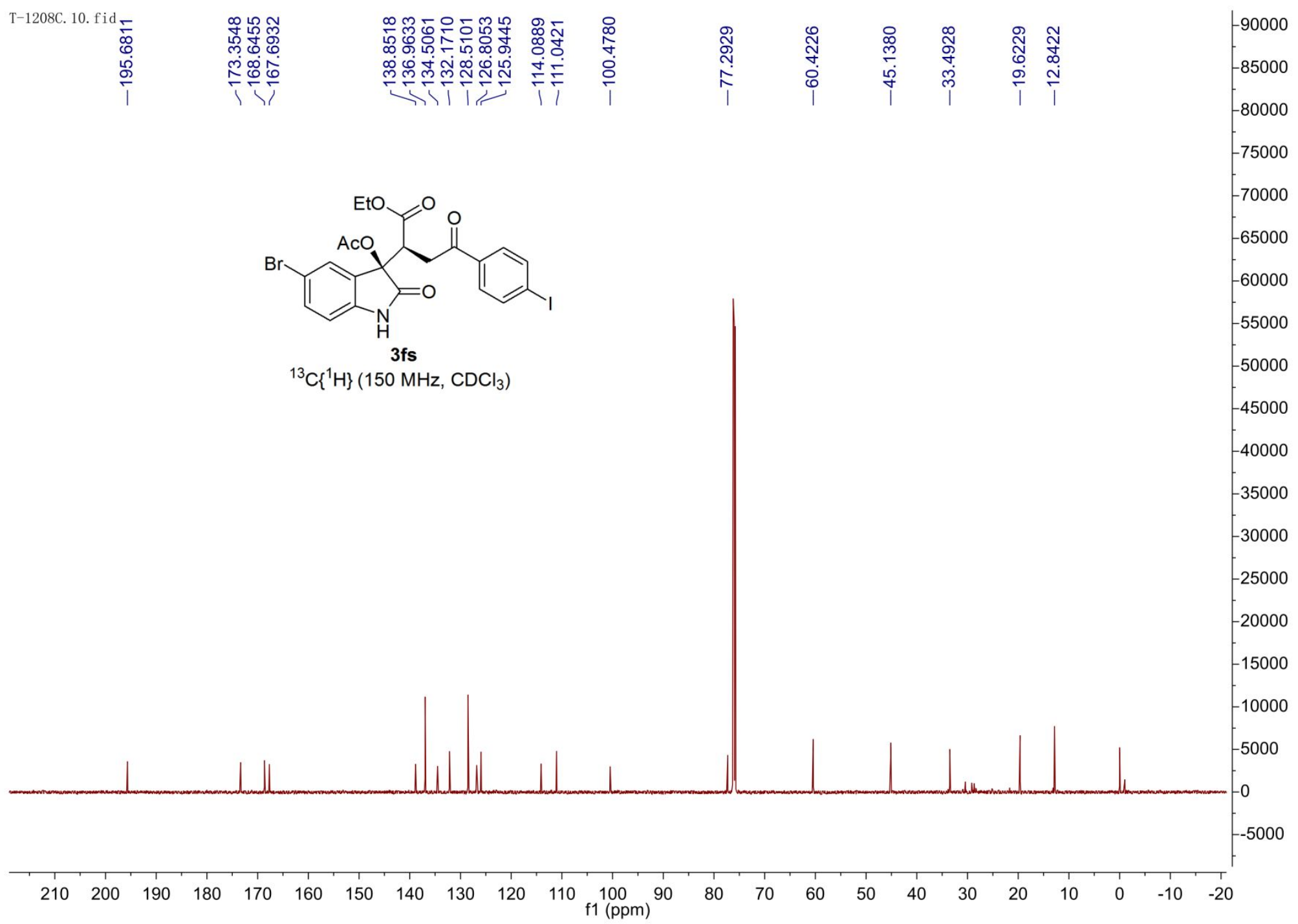


㒸

140000

130000

120000

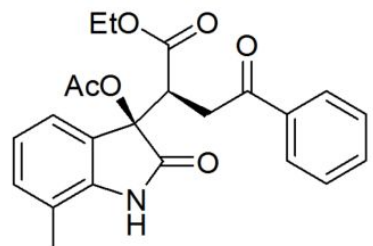

3 a

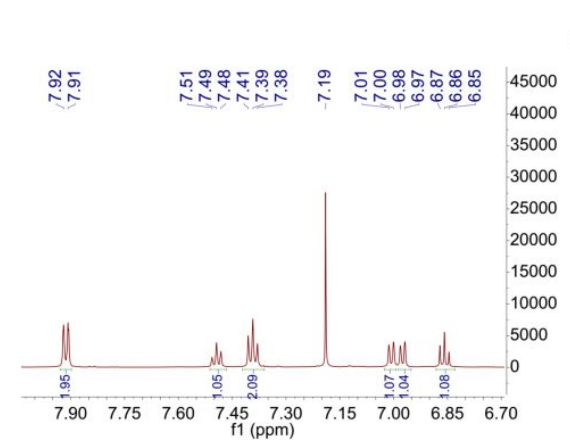

${ }^{1} \mathrm{H}\left(600 \mathrm{MHz}, \mathrm{CDCl}_{3}\right)$

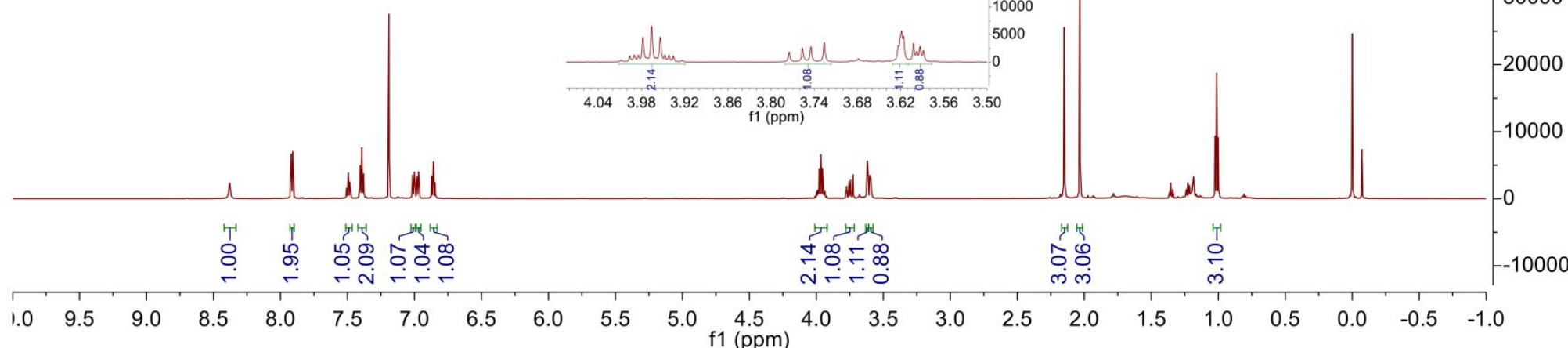




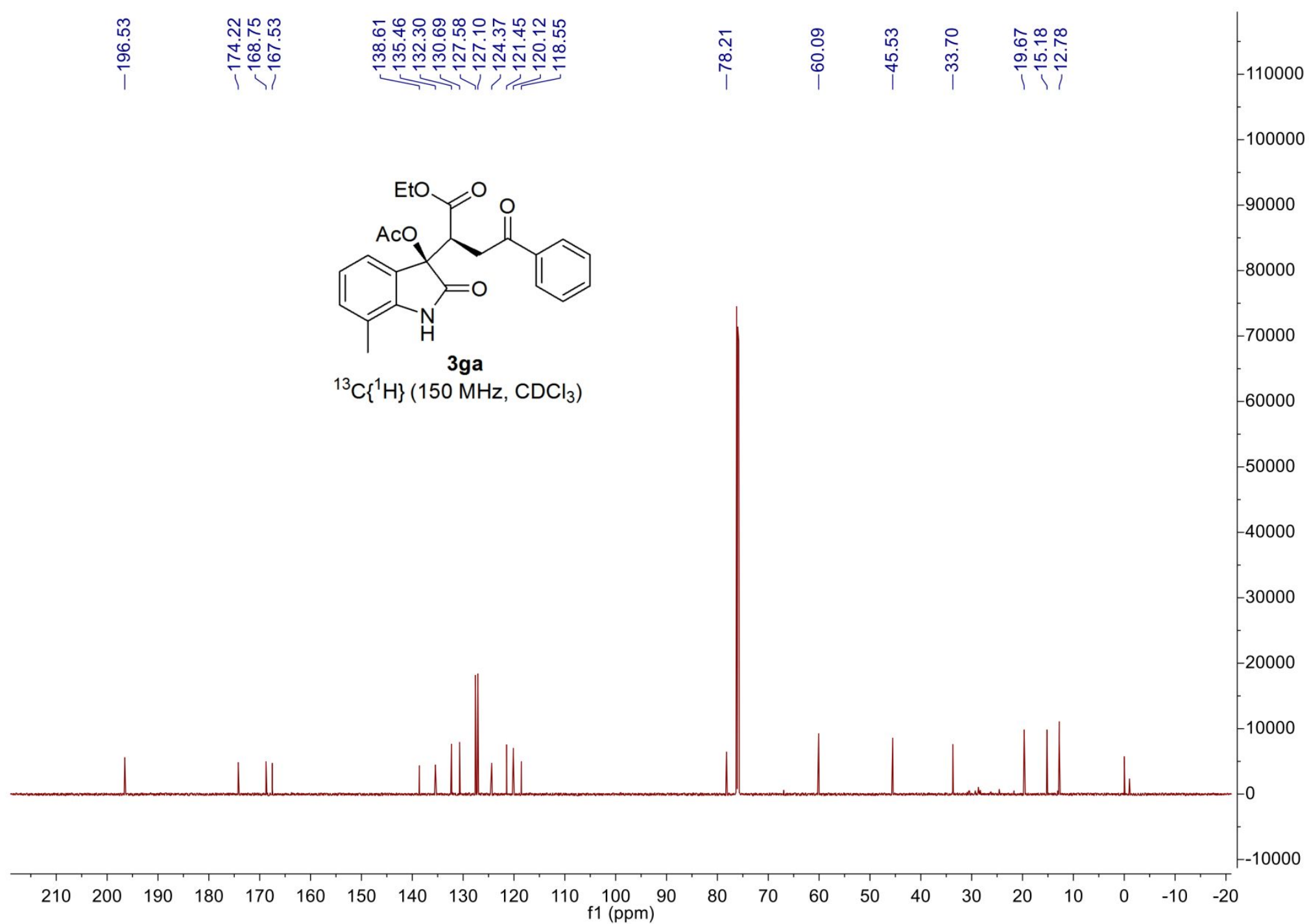




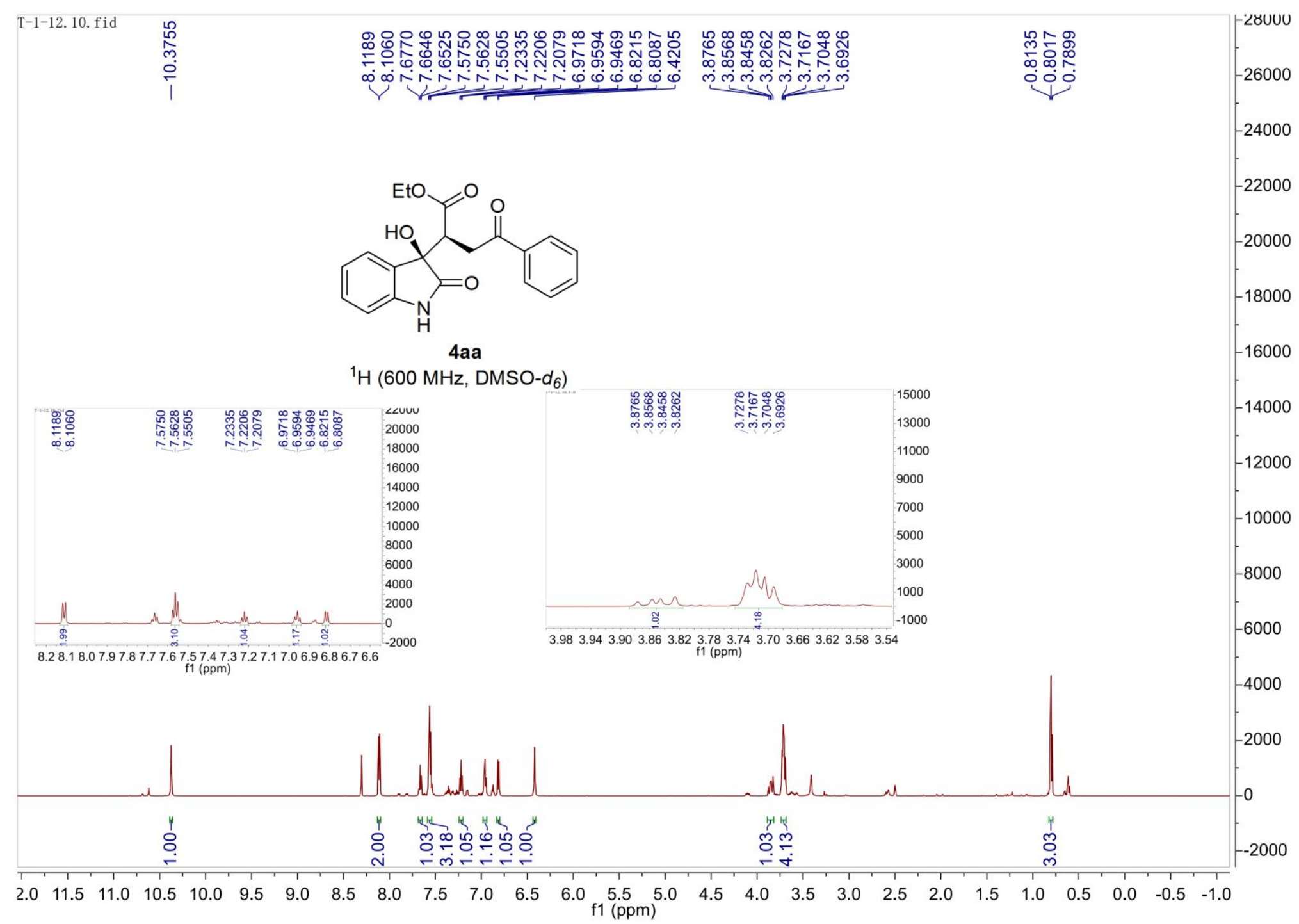




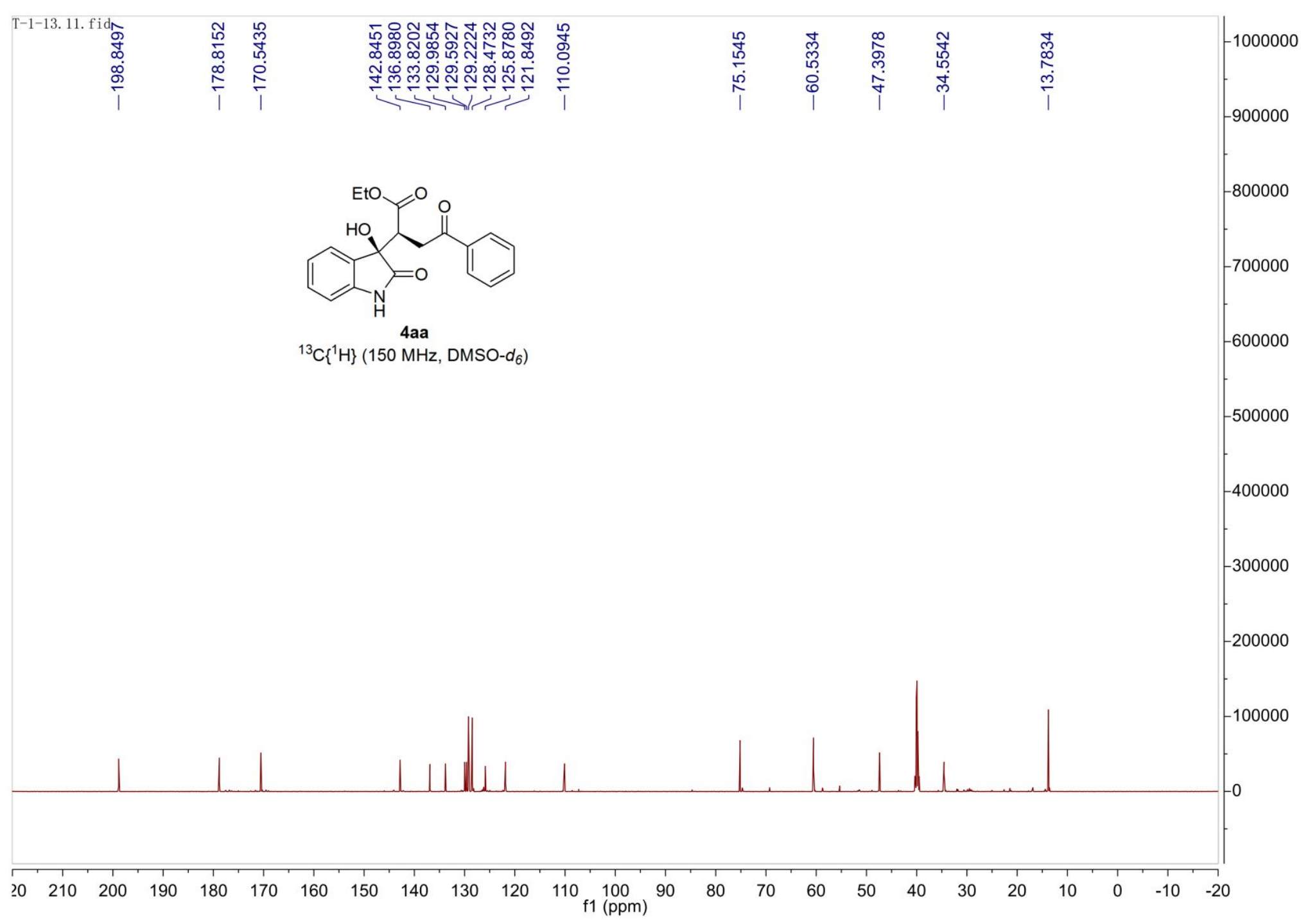




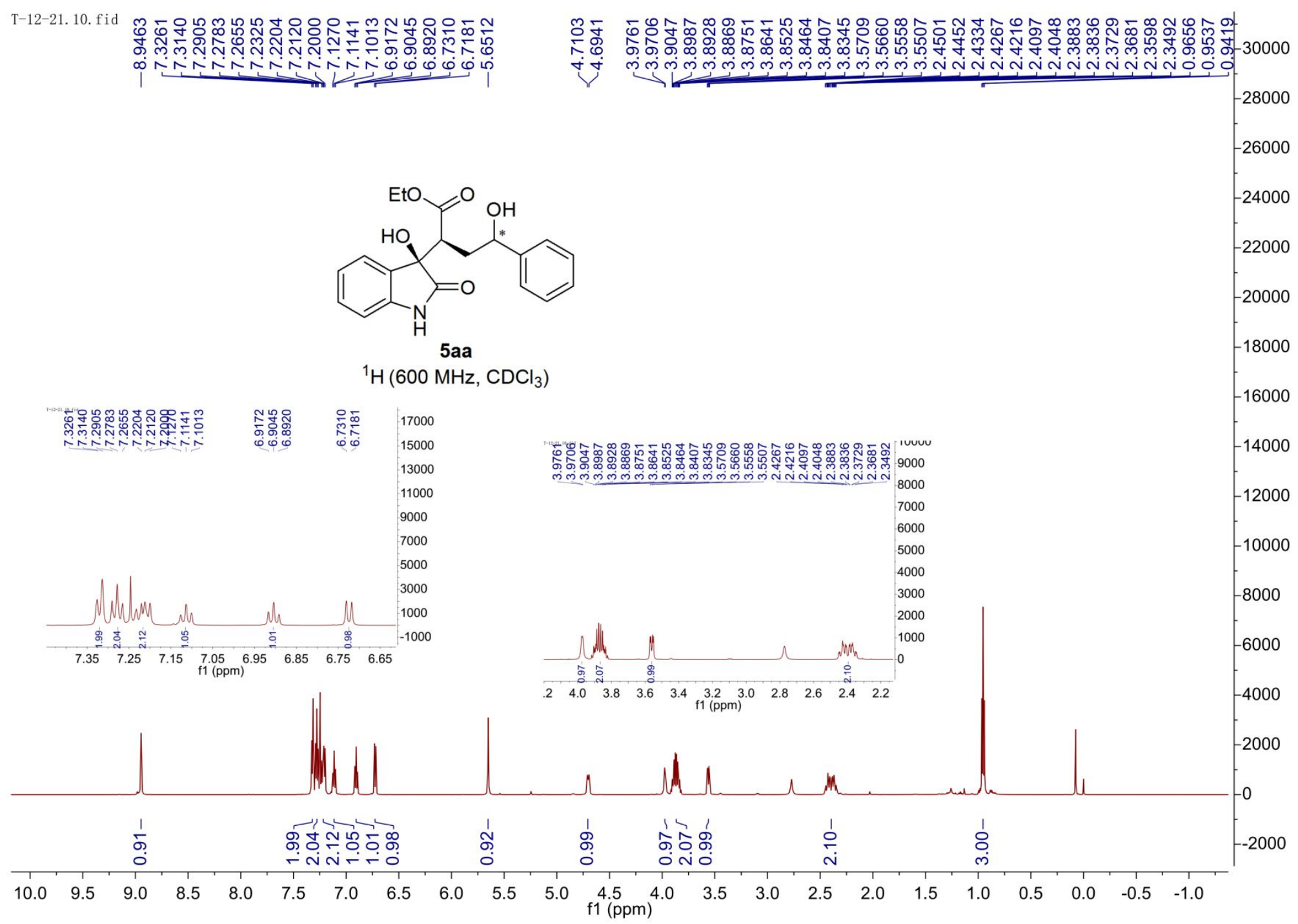




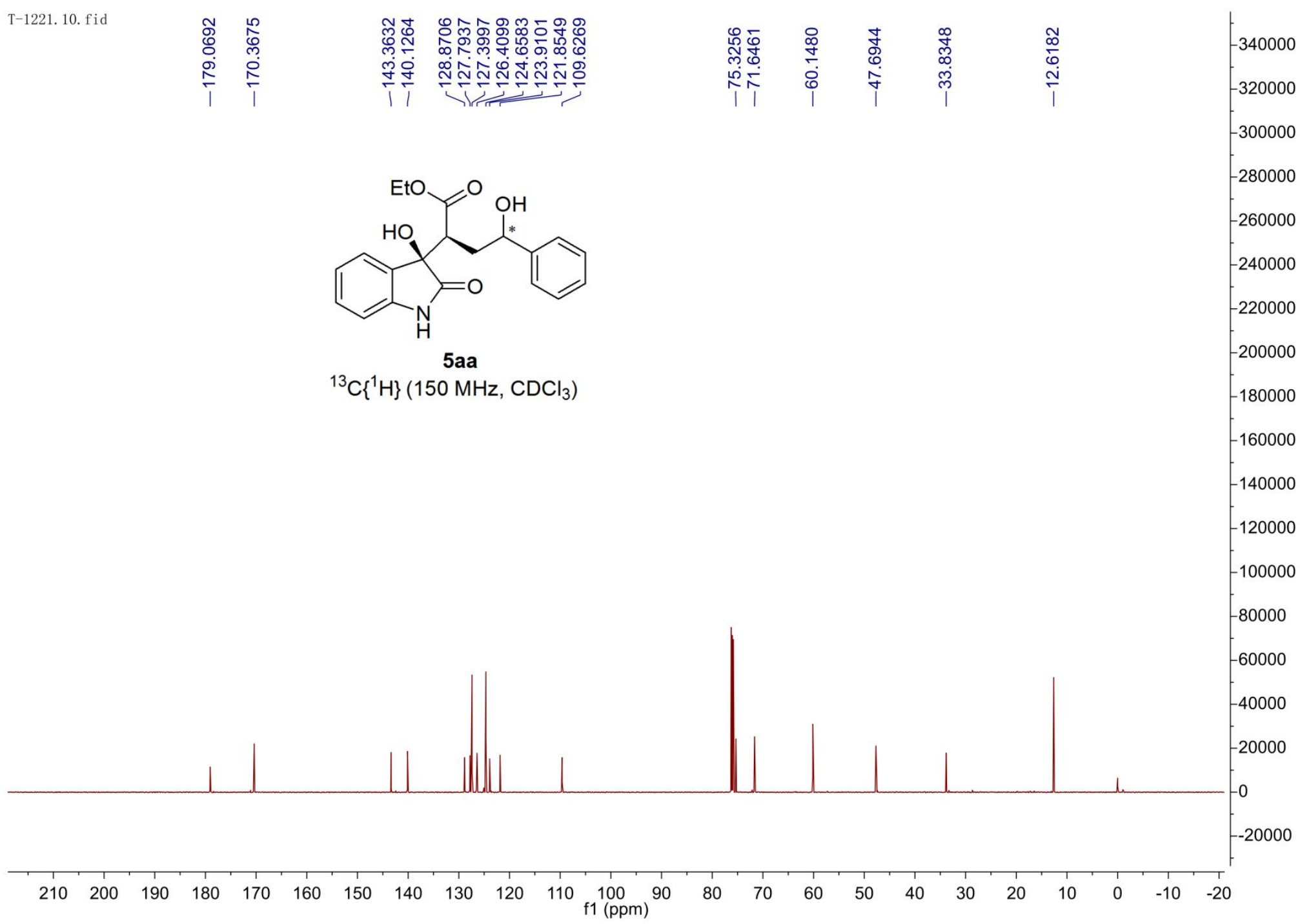




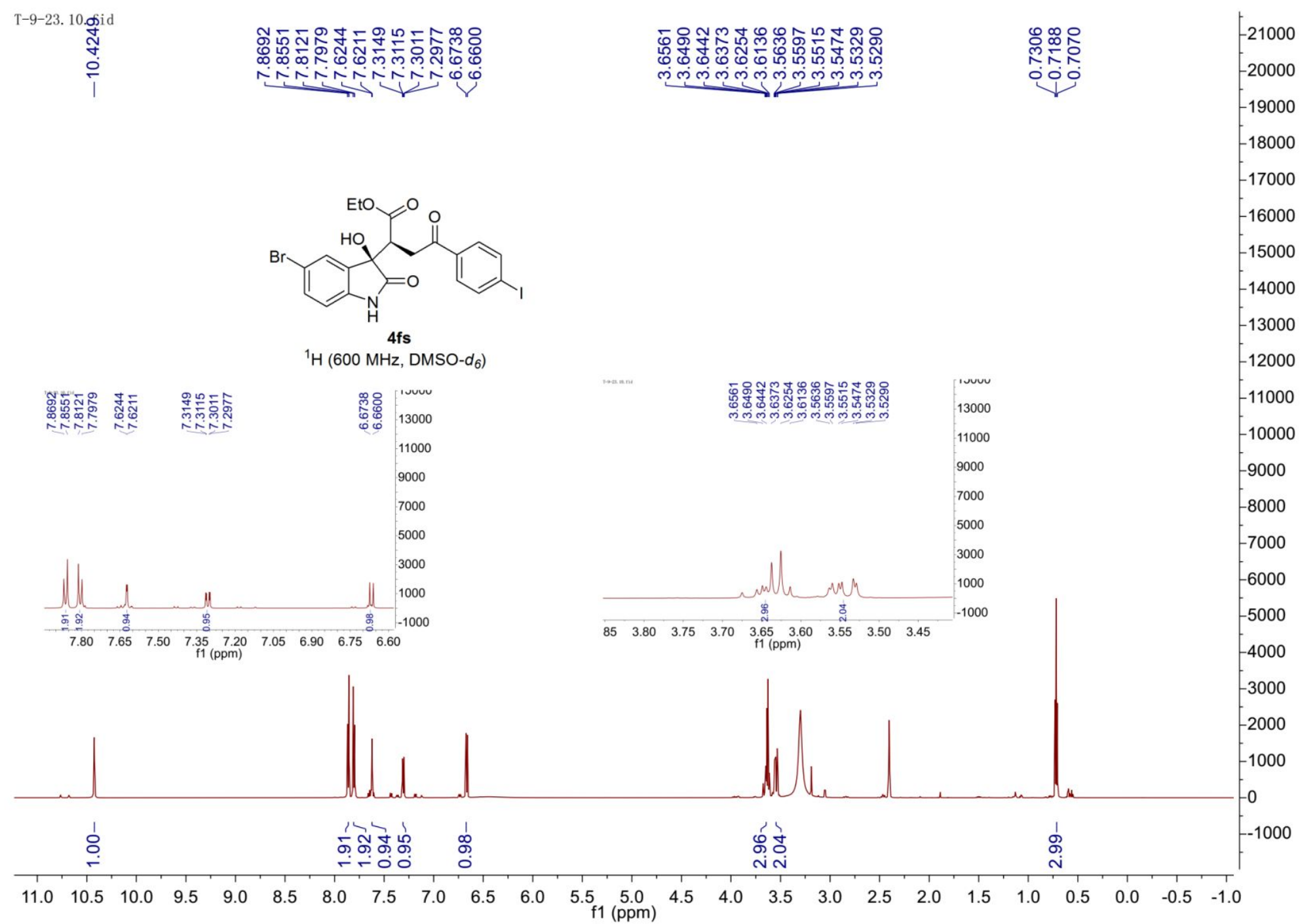




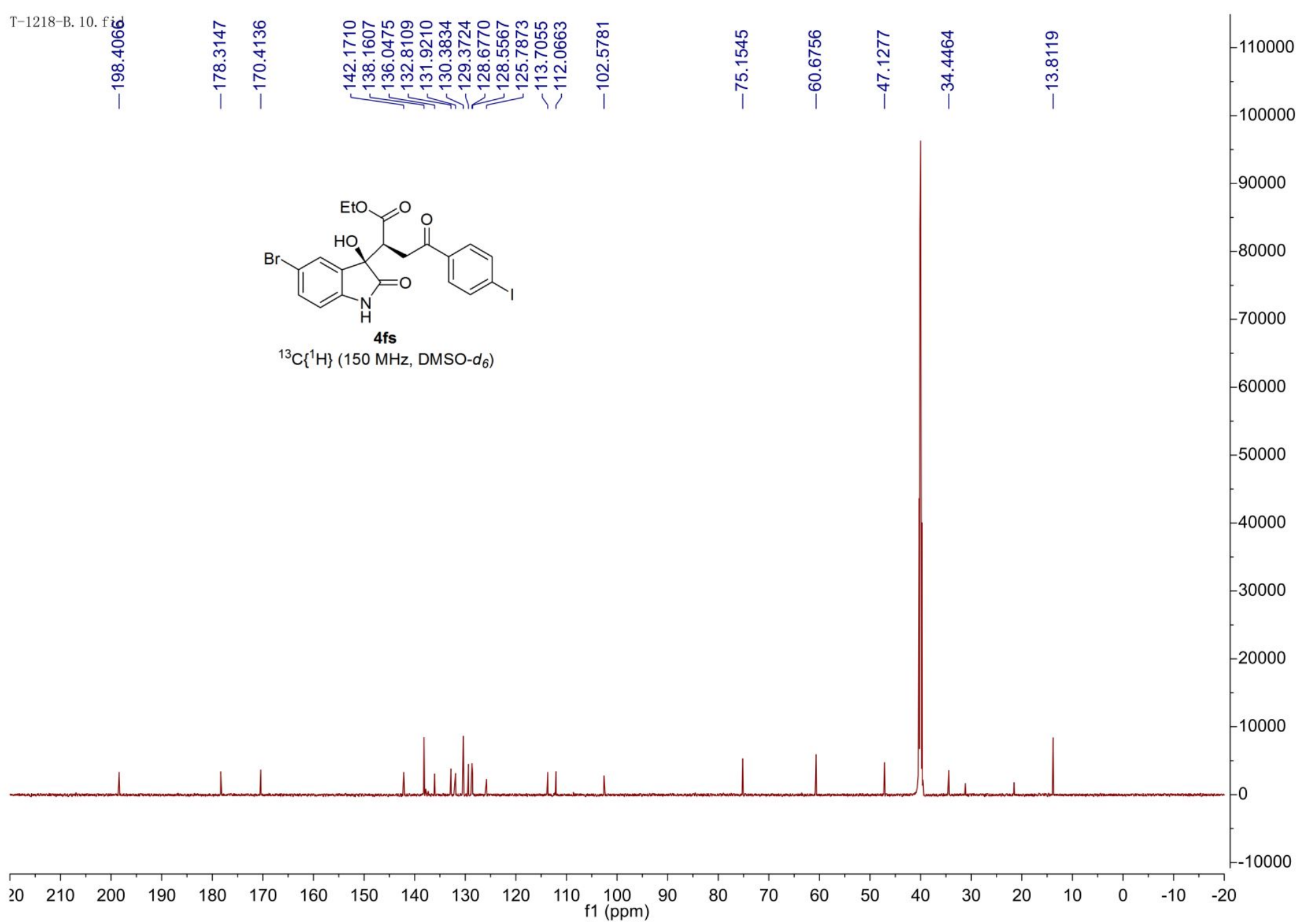

